\title{
A GENERIC FRAMEWORK FOR COLOUR TEXTURE SEGMENTATION
}

BY

PADMAPRIYA NAMMALWAR, B.Sc., M.Sc., M.Phil.,

This thesis is submitted to Dublin City University as the fulfilment OF THE REQUIREMENT FOR THE AWARD OF THE DEGREE OF

Doctor OF PHILOSOPHY

IN

ELECTRONIC ENGINEERING

SUPERVISED BY PROF.PAUL.F.WHELAN, B.Eng., M.Eng., Ph.D.,

School of EleCtronic EngineERING

Dublin City University

APRIL 2004 


\section{Declaration}

I hereby certify that this material, which I now submit for assessment on the programme of study leading to the award of Doctor of Philosophy, is entirely my own work and has not been taken from the work of others and to the extent that such work has been cited and acknowledged within the text of my work.

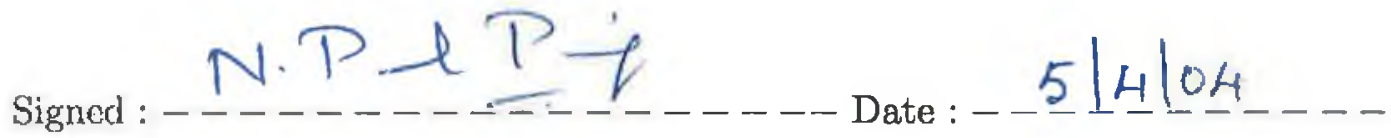
Padmapriya Nammalwar

ID: 50161431 
To my dear

Pappa, Amma, and Ammamma 


\section{Acknowledgements}

Many thanks to all who helped and encouraged me to complete this research work. In particular,

I would like to thank my supervisor Prof.Paul.F.Whelan for all the ideas, guidance and expertise provided by him throughout this research. My sincere gratitude to him for all his help.

I wish to thank the School of Electronic Engineering and Dublin City University for funding this research.

I wish to thank Dr.Ovidiu Ghita for his valuable suggestions during the implementation of the programming and for looking at this thesis for corrections and suggestions for improvement. I thank Pradeep for his valuable suggestions during the initial stages of this research.

I wish to thank all my collegues in Vision Systems Group, Robert Sadleir, Tarik Chowdhry, Micheal Lynch, John Mallon, Kevin Robinson and Nicholas Sezille. I thank collegues from the VRAL Lab Dr.Derek Molloy and John Higgins.

I wish to thank Robert Clare and Conor Maguire for the assistance given to me towards the computer maintenance.

Many thanks to my brothers Prasanth and Bhaskar for their constant support and encouragement during this research work.

Lastly, I would like to say my special thanks to Paddu for all his love, support and help. 


\begin{abstract}
This thesis proposes a novel method to combine the colour and the texture for colour texture segmentation. The objective of this research work is to derive a framework for colour texture segmentation and to determine the contribution of colour in colour texture analysis. The colour texture processing is based on the feature extraction from colour-textured images. The texture features were obtained from the luminance plane along with the colour features from the chrominance planes. Based on the above mentioned approach, a method was developed for colour texture segmentation. The proposed method unifies colour and texture features to solve the colour texture segmentation problem. Two of the grey scale texture analysis techniques, Local Binary Pattern (LBP) and Discrete Cosine Transform (DCT) based filter approach were extended to colour images. An unsupervised $k$-means clustering was used to cluster pixels in the chrominance planes. Non-parametric test was used to test the similarity between colour texture regions. An unsupervised texture segmentation method was followed to obtain the segmented image. The evaluation of the segmentation was based on the ROC curves. A quantitative estimation of colour and texture performance in segmentation was presented. The use of different colour spaces was also investigated in this study. The proposed method was tested using different mosaic and natural images obtained from VisTex and other predominant image database used in computer vision. The applications for the proposed colour texture segmentation method are, Irish Script On Screen (ISOS) images for the segmentation of the colour textured regions in the document, skin cancer images to identify the diseased area, and Sediment Profile Imagery (SPI) to segment underwater images. The inclusion of colour and texture as distributions of regions provided a good discrimination of the colour and the texture. The results indicated that the incorporation of colour information enhanced the texture analysis techniques and the methodology proved effective and efficient.
\end{abstract}




\section{Abbreviations and Symbols}

1D

2D

$3 \mathrm{D}$

CIE

CIE-XYZ

CIE-LAB

CIE-LUV

DCT

GIF

HSI

HSV

ISOS

JPEG

KS

LBP

$\mathrm{LBP} / \mathrm{C}$

LVQ

M-KS

MRF

MRMRF

MeasTex

NLC

NN

NTSC

RGB

ROC

$\mathrm{SAC}$
One Dimension

Two Dimension

Three Dimension

International Commission on Illumination

Non-uniform colour space, defined by CIE

Uniform colour space, defined by CIE

Uniform colour space, defined by CIE

Discrete Cosine Transform

Graphics Interchange Format

Hue, Saturation, Intensity, a colour model

Hue, Saturation, Value, a colour model

Irish Script on Screen

Joint Photographic Experts Group

Kolmogorov Smirnov

Local Binary Pattern

Local Binary Pattern and Contrast

Learning Vector Quantisation

Modified Kolmogorov Smirnov

Markov Random Field

Multi-resolution Markov Random Field

Measurement of Texture

Nearest linear Combination

Nearest Neighbour

National Television System Committee

Red, Green, Blue, a colour model

Receiver Operating Characteristic Curve

Symmetric auto-correlations 
SCOV

SLR

SPI

SRAC

SVR

TU

YIQ

YUV

VisTex

$V_{i}$

$E_{i}$

$u_{k}$

$E_{H_{x y}}$

$E_{V_{x y}}$

$E_{C_{x y}}$

$E_{D_{x y}}$

$E_{x y}$

$N_{x y}(R)$

$\mu_{x y}(R)$

$\sigma_{x y}^{2}(R)$

$\tilde{\sigma}_{x y}^{2}(R)$

$\sigma_{\max }^{2}(R)$

$\sigma_{\min }^{2}(R)$

$\Phi\left(\tilde{\sigma}_{x y}^{2}(R), \theta_{\sigma}\right)$

$\theta_{\sigma}$

$I_{x, y}^{(t+1)}$

$I_{x y}^{(t)}$
Symmetric auto-correlations with a covariance measure

Single Lens Reflex

Sediment Profile Imagery

Symmetric auto-correlations with rank order version

Symmetric auto-correlations with variance ratio

Texture Unit

Colour model used in American television transmission systems

Colour model used in European television transmission systems

Vision Texture

Pixels in the $3 \times 3$ neighbourhood

Threshold value in the $3 \times 3$ neighbourhood

Basis Vector

Local discontinuity along horizontal direction

Local discontinuity along vertical direction

Local discontinuity along counter-diagonal direction

Local discontinuity along diagonal direction

Local discontinuity measure based on the four detectors

Contextual neighbourhood associated with pixel (x, y)

Mean of pixels on $N_{x y}(R)$

Spatial variance of pixels on $N_{x y}(R)$

Normalised spatial variance

Maximal spatial variance across the image

Minimal spatial variance across the image

Transformation based on the normalised variance

Threshold to limit the degree of contextual discontinuities

Feature preserving adaptive smoothing

Intensity of the pixel $(\mathrm{x}, \mathrm{y})$ at iteration $t$ 
$\eta_{i j}$

$\gamma_{i j}^{(t)}$

$\alpha$

S

$\mathrm{P}(\mathrm{i}, \mathrm{j})$

G

$f_{i}$

$s, m$

$\mathrm{D}(\mathrm{s}, \mathrm{m})$

$F_{s}(i), F_{m}(i)$

$G_{\max }$

$G_{m i n}$

$\mathrm{R}$

$\mathrm{X}$

$S_{\min }$

MI

MIR

$\mathrm{Y}$

r

d

$w_{1}, w_{2}$

$M K S_{1}, M K S_{2}$
Encodes the effect of contextual discontinuities

Encodes the effect of local discontinuities

To determine the extent of feature preservation in terms of contextual discontinuities

To determine the preservation extent of local discontinuities

The mean of the colour plane

pixel value at the position $(i, j)$

Log-likelihood pseudo metric G-statistic, similarity measure

The frequency at bin $\mathrm{i}$

Two sample histograms

Discrepancy statistic or Modified Kolmogorov Smirnov statistic

Sample cumulative distributions

Maximum $G$ value

Minimum $G$ value

Ratio of the maximum and minimum $\mathrm{G}$ value

Threshold value in splitting

Minimum block size

Merger Importance value

Merger Importance ratio

Threshold value in merging

a disc with radius $r$ on the pixelwise classification

a square with a dimension $d$ on the boundary refinement

Weights according to the distribution of colour clustered labels

Modified Kolmogorov Smirnov statistic for the intensity histogram and the colour histogram respectively 


$\begin{array}{ll}\text { MinMI } & \text { Minimum of merger importance value } \\ k_{j} & \text { Uniformity factor of the sample regions } \\ C L_{j}[i] & \text { Histogram of the colour clustered labels in the sample re- } \\ & \text { gions } \\ N_{p} & \text { Number of pixels in the corresponding regions } \\ e & \text { Ratio between the number of pixels incorrectly segmented } \\ & \text { by the total number of pixels in the region }\end{array}$




\section{List of Figures}

1.1 (a), (b) - Micro textures (c), (d) - Macro textures ....... 5

1.2 (a), (c) - Input Image (b), (d) - Synthesised Image [15] . . . . 7

1.3 (a) Bark (b) Brick (c) Sand (d) Fabric. Different types of VisTex $[27]$ textures and their identities . . . . . . . . . . 8

1.4 Different homogeneous textures were labelled in texture classification 8

1.5 Texture segmentation - An example ............ 9

2.1 Section summary - Texture segmentation . . . . . . . . 22

2.2 Section summary - Texture Classification . . . . . . . . . 28

2.3 Section summary - Texture Classification . . . . . . . . . 29

2.4 Section summary - Related texture based approaches . . . . . . 34

2.5 Scction summary - Related texture based approaches ... . . . 35

2.6 Section summary - Colour segmentation . . . . . . . . 40

2.7 Section summary - Colour texture segmentation . . . . . . . . 44

2.8 Section summary - Colour texture segmentation ........ 45

2.9 Section summary - Colour texture classification . . . . . . . 49

2.10 Section summary - Colour texture classification . . . . . . 50

3.1 Steps in the calculation of LBP and $\mathrm{C}$ value ........ 59 
3.2 k-means clustering method. (a) represents the original image with the different patterns and the centroid of the image data. (b) illustrates the cluster membership after the first iteration. (c) shows the cluster membership after the second iteration. (d) represents the final clustered image. . . . . . . . . . . . . . . . 63

3.3 A typical example of the splittree and the corresponding image

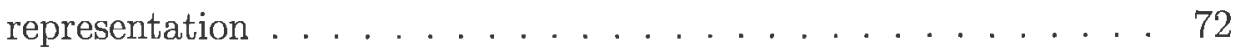

3.4 Pseudo code for the splittree approach . . . . . . . . . . . 73

3.5 A typical example of mergegraph . . . . . . . . . . . . . . . . 75

3.6 Pseudo code for the mergegraph approach . . . . . . . . 76

4.1 Flowchart representing feature extraction approaches and the colour texture segmentation methods . . . . . . . . . . . 80

4.2 The colour texture segmentation for Method-I . . . . . . . . . 81

4.3 The colour texture segmentation for Method-II . . . . . . . . . . 84

4.4 The colour texture segmentation for Method-III . . . . . . . . . 89

5.1 Sample segmented results from greyscale images in Method-I using $\mathrm{LBP} / \mathrm{C} \ldots \ldots \ldots \ldots \ldots \ldots$

5.2 ROC curves representing the performance of grey scale $\mathrm{LBP} / \mathrm{C}$ and $\mathrm{DCT} \ldots \ldots \ldots \ldots \ldots \ldots$

5.3 (a) - (d) Segmented results of a mosaic in grey scale and R, G and B planes respectively using $\mathrm{LBP} / \mathrm{C} \ldots . . . . . . . . . .98$

5.4 (a) - (d) Segmented results of bird in greyscale and R, G and B planes respectively using $\mathrm{LBP} / \mathrm{C} \ldots \ldots 9$

5.5 Sample segmented results of mosaic and natural images from MethodI using DCT . . . . . . . . . . . . . . . . . . . . 101

5.6 (a), (c), (e) and (g) shows the segmented results. (b), (d), (f) and (h) represents the corresponding pixelwise classified images . . . . 102 
5.7 Sample segmented results from Method-II using $\mathrm{LBP} / \mathrm{C}$ and a chromaticity feature in RGB space . . . . . . . . . . . . . . . 104

5.8 Sample segmented results from Method-II using DCT and a chromaticity feature in RGB space . . . . . . . . . . . . 105

5.9 ROC curves for LBP/C approach in greyscale and $\mathrm{LBP} / \mathrm{C}$ with statistical colour feature standard deviation in RGB space . . . 106

5.10 ROC curves for DCT approach in greyscale and DCT with statistical colour feature standard deviation in RGB space . . . . . . 107

5.11 Bar chart representing the importance of chromaticity features using the LBP /C approach . . . . . . . . . . . . . 108

5.12 Bar chart representing the importance of chromaticity features using the DCT approach . . . . . . . . . . . . . . . 109

5.13 Comparison of the colour spaces in LBP/C . . . . . . . . . 109

5.14 Comparison of the colour spaces in DCT . . . . . . . . . . 110

5.15 Comparison of the different approaches in Method-II . . . . . 110

5.16 Influence of the colour and texture . . . . . . . . . . . 112

5.17 Sample segmented results of natural images . . . . . . . . . 113

5.18 Sample results illustrates the poor performance of the pixelwise classification . . . . . . . . . . . . . . 115

5.19 ROC curves for LBP/C in greyscale and LBP/C with colour clustering in RGB space . . . . . . . . . . . . . . 117

5.20 ROC curves for DCT in greyscale and DCT with colour clustering in RGB space . . . . . . . . . . . . . . . . . . 118

5.21 Bar chart representing the performance of the two approaches in RGB, YIQ and HSI colour space . . . . . . . . . . . . . 119

5.22 Comparison of different approaches in Method-III . . . . . . . . 120 
5.23 (a), (d), (g) Segmentation based on colour, (b), (e), (h) Segmentation based on texture, (c), (f), (i) Segmentation based on colour

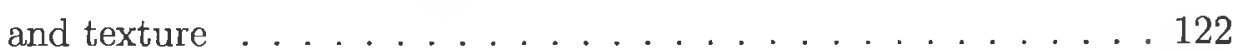

5.24 Sample segmented results from Method-III using LBP with colour clustering . . . . . . . . . . . . . . . . . . . . . 124

5.25 Sample segmented results from Method-III using LBP with colour clustering . . . . . . . . . . . . . . . . 125

5.26 Sample segmented results from Method-III using DCT with colour clustering . . . . . . . . . . . . . . . 126

5.27 Segmented results of natural images from Method-III using LBP/C with colour clustering . . . . . . . . . . . . . . . 127

5.28 Segmented results of natural images from Method-III using DCT with colour clustering . . . . . . . . . . . . . . 128

5.29 The influence of adaptive smoothing . . . . . . . . . . . 130

5.30 The influence of noise, $a-1 \%, b-2 \%, c-4 \%$, in the colour texture segmentation ......................... 131

5.31 Segmentation effect of the rotated images . . . . . . . . . . 132

5.32 Comparison of Method-II using LBP/C with colour features and Method-III using LBP/C with colour clustering . . . . . . . . 135

5.33 The executable file for Method-I . . . . . . . . . . . . 136

5.34 The executable file for Method-II using LBP/C . . . . . . . 137

5.35 The executable file for Method-II using DCT . . . . . . . . . 139

5.36 The executable file for Method-III . . . . . . . . . . . . . 139

6.1 (1a), (2a), (3a) and (4a) represents segmented results of ISOS images using Method-III, (1b), (2b), (3b), (4b) represents results of ISOS images using Method-III after the boundary refinement stage . . . . . . . . . . . . . . . . . . 143 
6.2 (1a), (2a), (3a), (4a), (5a) represents segmented results of skin cancer images from Method-III, (1b), (2b), (3b), (4b), (5b) represents the results after the boundary refinement stage . . . . . 146

6.3 (1a), (2a) represents segmented results of SPI images using MethodIII, (1b), (2b) represents segmentation results after the boundary refinement stage . . . . . . . . . . . . . . . . . 148

B.1 RGB Colour Cube . . . . . . . . . . . . . . . . . 180

B.2 Hexagonal Pyramid . . . . . . . . . . . . . . . 181

B.3 CIE chromaticity diagram [99] . . . . . . . . . . . 184

B.4 Complementary colour pairs . . . . . . . . . . 186

C.1 Different classes used in Method-II . . . . . . . . . . . 188

C.2 Different classes used in Method-III . . . . . . . . . . . . 189

D.1 M1-M6 are the mosaic images constructed from VisTex database 190

D.2 M7-M18 are the mosaic images constructed from VisTex database 191

D.3 M19-M30 are the mosaic images constructed from VisTex database 192

D.4 M31-M37 are the mosaic images from VisTex database, M38 and M39 were mosaic images used by Mirmehdi and Petrou [86] . . . 193

D.5 N1-N9 are the natural images from VisTex database, N10 and N11 are the natural images which were used by Panjwani and Healey [24] . . . . . . . . . . . . . . . . . . . . 194

E.1 Sample segmented results of mosaic images from Method-I using $\mathrm{LBP} / \mathrm{C}$ in different colour planes . . . . . . . . . . . . . 195

E.2 Sample segmented results of mosaic and natural images from MethodI using LBP/C in different colour planes . . . . . . . . . . . 196

F.1 Sample segmented results from Method-II using LBP/C and colour features . . . . . . . . . . . . . . . . 197 
F.2 Sample segmented results from Method-II using LBP/C and colour features . . . . . . . . . . . . . . . . . . 198

F.3 Sample segmented results from Method-II using DCT and colour

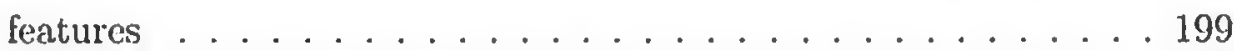

G.1 Sample segmented results from Method-III using LBP/C with colour clustering . . . . . . . . . . . . . . . 200

G.2 Sample segmented results from Method-III using LBP/C with colour clustering . . . . . . . . . . . . . . . . 201

G.3 Sample segmented results of natural images from Method-III using LBP/C with colour clustering . . . . . . . . . . . . . 202

G.4 Sample segmented results from Method-III using DCT with colour clustering . . . . . . . . . . . . . . . . 202

G.5 Sample segmented results from Method-III using DCT with colour

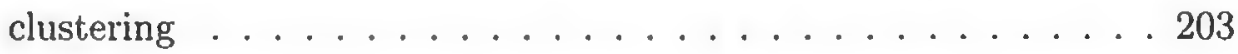

G.6 Sample segmented results of mosaics and natural images from Method-III using DCT with colour clustering . . . . . . . . 204 


\section{List of Tables}

5.1. Percentage of segmented results - Significance of colour features in colour texture analysis . . . . . . . . . . . . . 108

5.2 Quantitative evaluation for different block size . . . . . . . 116

5.3 The performance of the $\mathrm{LBP} / \mathrm{C}$ with colour clustering and DCT with colour clustering in RGB, YIQ and HSI spaces as percentage of segmented results . . . . . . . . . . . . . . 119

5.4 Average segmentation error (\%) depending on the colour and the

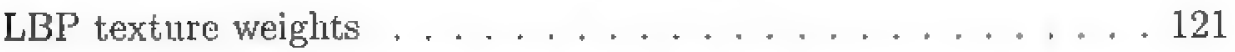

5.5 Average segmentation error (\%) depending on the colour and the DCT texture weights . . . . . . . . . . . . . . 121 


\section{Contents}

1 Introduction 1

1.1 Research Objective and Motivation .............. 1

1.2 Definitions of Texture, Colour and Colour Texture . . . . . . . 3 3

1.3 Study of Textures ....................... 6

1.4 Colour Analysis . . . . . . . . . . . . . . . . . 12

1.5 Issues in Colour Texture Segnentation . . . . . . . . . . . 13

1.6 Organisation of the Thesis . . . . . . . . . . 16

1.7 Summary .................................... 17

2 Review of Related Work $\quad 18$

2.1 Introduction . . . . . . . . . . . . . . . 18

2.2 Review of Texture Studies . . . . . . . . . . . . . 18

2.2 .1 Texture Segmentation ................... 18

2.2 .2 Texture Classification . . . . . . . . . . . 23

2.2.3 Rolated Texture Based Approaches . . . . . . . . . 30

2.3 Review of Colour Image Segmentation . . . . . . . . 36

2.4 Review of Colour Toxture Research . . . . . . . . . . . . 41

2.4.1 Colour Texture Segmentation ............. 41

2.4 .2 Colour Texture Classification . . . . . . . . . . 46

2.4.3 Colour Texture Image Rotrieval . . . . . . . . . 51

2.4 .4 Colour Texture Applications . . . . . . . . . . . 52 
2.4.5 Related Colour Texture Based Approaches . . . . . . . 53

2.5 Summary . . . . . . . . . . . . . . . . . . . 55

3 Image Processing Analysis Techniques $\quad 57$

3.1 Introduction . . . . . . . . . . . . . . 57

3.2 Feature Extraction Techniques . . . . . . . . . . . 57

3.2 .1 Texture Feature Extraction Techniques . . . . . . . . 58

3.2.2 Colour Feature Extraction Techniques ........ . 61

3.3 Selection of Colour Space . . . . . . . . . . . . . 64

3.4 Adaptive Smoothing . . . . . . . . . . . . . . 65

3.5 Non-parametric Test . . . . . . . . . . . . . . . . . 68

3.5 .1 G-statistic . . . . . . . . . . . . . . . . 69

3.5.2 Modified-Kolmogorov Smirnov (M-KS) . . . . . . . . 69

3.6 Unsupervised Texture Segmentation Method . . . . . . . . 70

3.7 Boundary Refinement . . . . . . . . . . . . . . 77

3.8 Summary . . . . . . . . . . . . . . . . . . . . . 78

$\begin{array}{llr}4 & \text { Methodology } & \mathbf{7 9}\end{array}$

4.1 Introduction . . . . . . . . . . . . . . . . 79

4.2 Colour Texture Segmentation Methods and Description . . . . 80

4.2.1 Colour Texture Segmentation - Method-I . . . . . . . . . 80

4.2.2 Colour Texture Description for Method-I . . . . . . . . . . 82

4.2.3 Colour Texture Segmentation - Method-II . . . . . . . 83

4.2.4 Colour Texture Description for Method-II . . . . . . . . 85

4.2.5 Colour Texture Segmentation - Method-III . . . . . . . . 88

4.2.6 Colour Texture Description for Method-III . . . . . . . . 90

4.3 Summary . . . . . . . . . . . . . . . . . . . 93

5 Results and Discussion $\quad 94$

5.1 Introduction . . . . . . . . . . . . . . . . . . . . . . 94 
5.2 Segmentation Results Using Method-I . . . . . . . . . . . . 95

5.3 Segmentation Results Using Method-II . . . . . . . . . . . 103

5.3 .1 Performance Evaluation . . . . . . . . . . . 105

5.3.2 Influence of Texture and Colour . . . . . . . . . . 111

5.4 Segmentation Results Using Method-III . . . . . . . . . . . 116

5.4 .1 Performance Evaluation . . . . . . . . . 116

5.4 .2 Influence of Texture and Colour . . . . . . . . . 123

5.5 Discussion . . . . . . . . . . . . . . . . . . . . . 134

5.6 Algorithm Implementation . . . . . . . . . . . . 136

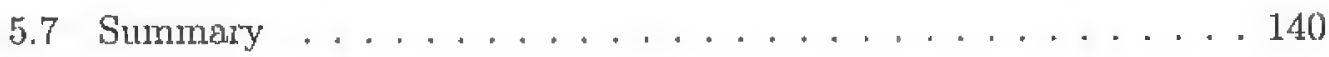

6 Application of the Developed Colour Texture Segmentation Method$\begin{array}{ll}\text { ology } & 141\end{array}$

6.1 Introduction . . . . . . . . . . . . . . 141

6.2 Colour Texture Segmentation Applications . . . . . . . . . . 142

6.2.1 Irish Script on Screen Images . . . . . . . . . . . . 142

6.2 .2 Skin Cancer Images . . . . . . . . . . . . . . . 144

6.2 .3 Sediment Profile Imagery . . . . . . . . . . . . . 147

6.3 Summary ............................ 150

$\begin{array}{llr}7 \text { Conclusions } & 151\end{array}$

8 Thesis Contribution and Future Work $\quad 156$

8.1 Thesis Contribution . . . . . . . . . . . . . 156

8.2 Future Work . . . . . . . . . . . . . . . . 159

$\begin{array}{ll}\text { Bibliography } & 161\end{array}$

$\begin{array}{lr}\text { List of Publications } & 172\end{array}$

$\begin{array}{ll}\text { Appendix A } & 173\end{array}$ 
A Texture Feature Extraction Techniques 173

A.1 Co-occurrence . . . . . . . . . . . . . . . . 173

A.2 Gabor Filters . . . . . . . . . . . . . . . . 175

A.3 Markov Random Field . . . . . . . . . . . . . . . . 176

A.4 Fractals ........................ 177

$\begin{array}{lr}\text { Appendix B } & 179\end{array}$

$\begin{array}{lr}\text { B Colour Spaces } & 179\end{array}$

B.1 RGB Space . . . . . . . . . . . . . . . . . . 179

B.2 HSI Space . . . . . . . . . . . . . . . . . . . . 180

B.3 HSV Space . . . . . . . . . . . . . . . . . 181

B.4 YIQ Space . . . . . . . . . . . . . . . . 182

B.5 YUV Space . . . . . . . . . . . . . . . . . 183

B.6 CIE-XYZ Space . . . . . . . . . . . . . . . 183

B.7 CIE-LUV Space . . . . . . . . . . . . . . . . . 185

B.8 CIE-LAB Space . . . . . . . . . . . . . . . 186

$\begin{array}{ll}\text { Appendix C } & 188\end{array}$

$\begin{array}{lr}\text { C Implementation } & 188\end{array}$

$\begin{array}{ll}\text { Appendix D } & 190\end{array}$

$\begin{array}{ll}\text { D Database Images } & 190\end{array}$

D.1 Database of Mosaic Images . . . . . . . . . . . . . 190

D.2 Database of Natural Images . . . . . . . . . . . . . 194

$\begin{array}{lr}\text { Appendix E } & 195\end{array}$

$\begin{array}{lr}\text { E Results - Method-I } & 195\end{array}$

$\begin{array}{lr}\text { Appendix F } & 197\end{array}$ 
$\begin{array}{llr}\text { F Results - Method-II } & 197\end{array}$

F.1 Results using $\mathrm{LBP} / \mathrm{C}$ and colour features . . . . . . . . . 197

F.2 Results using DCT and colour features . . . . . . . . . . . 199

$\begin{array}{lr}\text { Appendix G } & \mathbf{2 0 0}\end{array}$

G Results - Method-III 200

G.1 Results using LBP/C with colour clustering . . . . . . . . . 200

G.2 Results using DCT with colour clustering . . . . . . . . . . 202 


\section{Chapter 1}

\section{Introduction}

\subsection{Research Objective and Motivation}

Most natural surfaces exhibit textures. Texture is observed as structural patterns in the grain, grass, cloth, wood, brick and sand. It is an important characteristic used in interpreting the image information. Texture refers to the pattern of intensity variations in an image and has varying degrees of randomness and regularity. The coarseness or fineness of textured images are relative and depends on the scale of the image. For example in examining an image from a large distance the image may appear smooth, without any distinguished textures. On decreasing the distance, the texture appears fine and if the distance is further decreased the texture may appear coarse. Hence texture description is scale dependent.

Colour is a property of enormous importance to human visual perception and is a rich source of information for image analysis. It is an intrinsic attribute of an image and provides more information than a single intensity value. In computer vision, colour is used in image segmentation, image classification and image database retrieval. Human perception of colour depends on the spatial frequency of the colour component. Specifically, the perceptual response of the 
human visual system to a certain part of the electromagnetic spectrum depends on the frequency with which this stimulus is spatially distributed. Hence, colours in multi-colour pattern are perceived differently than from uniform areas [1].

Texture and colour are widely accepted as two key factors in image analysis. Texture and colour properties have been regarded separately rather than collectively. Texture and colour are inseparable, since textures have a colour aspect and coloured surfaces are textured. Most work in the past on texture analysis and segmentation derived structural description for texture, example coarseness, regularity, blobiness, orientation etc., with the colour information used as an extra cue [1]. Recently, several studies were directed to the problem of joint representation of texture and colour. Numerous approaches for texture analysis and colour analysis have been developed. Though colour and texture are inherently related to each other there does not yet exist a reliable method to combine both colour and texture for colour texture segmentation. Hence, the main objective of this research work is to find a methodology for colour texture segmentation and to examine the contribution of colour in the analysis of textures i.e., to determine the use of colour information in the colour texture segmentation process.

Colour texture analysis plays a vital role in many applications of computer vision. Important applications include defect detection in industrial surface inspection, detection of defects in textile and paint inspection, assessment of carpet wear in quality control and detection of damage regions in script images. In addition, colour texture analysis is used in remote sensing for ground classification, segmentation of satellite or aerial images, segmentation of underwater images and in several medical applications. Image textures are used in combination with colour features to diagnose leukemic malignancy in samples of stained blood cells [2]. Colour textures are used in the classification of blood cells and in the segmentation of skin cancer images for disease detection in biomedical image processing. 
Some other useful applications are, the segmentation of textured regions in document analysis, to find the quality of the food in food industries, and in image retrieval for classification [3]. The existence of a variety of applications is the motivating factor for this research work. The applications involved in this research work are the segmentation of script images, skin cancer images and the underwater images.

\subsection{Definitions of Texture, Colour and Colour Texture}

\section{Definition of Texture}

Extensive work has been carried out in the area of texture analysis for several decades and many papers have been published, but there does not exist a widely accepted definition of texture. Formulation of a mathematical description to define a texture has not been carried out so far due to the variation in the textures.

Some definitions of texture from image processing handbooks and well known research publications are as follows:

- Sklansky-Image Segmentation and Feature Extraction [4] A region in an image has a constant texture if a set of local statistics or other local properties of the picture function are constant, slowly varying or approximately periodic.

- Wilson and Spann-Image Segmentation and Uncertainty [5] Textured regions are spatially extended patterns based on the more or less accurate repetition of some unit cell called texton or subpatterns.

- A.K.Jain-Fundamentals of Digital Image Processing [6]

The term texture generally refers to the repetition of basic texture elements 
called texels. The texel contains several pixels, whose placement could be periodic, quasi-periodic or random. Natural textures are generally random, whereas artificial textures are often deterministic or periodic. Texture may be coarse, fine, smooth, granulated, rippled, regular, irregular or linear.

\section{- IEEE Standard Glossary of Image Processing and Pattern Recog-} nition Terminology [7]

Texture is an attribute representing the spatial arrangement of the grey levels of the pixels in a region.

\section{- R.C.Gonzalez and R.E.Woods-Digital Image Processing [8]}

We intuitively view texture as a descriptor that provides a measure of properties such as smoothness, coarseness and regularity.

- R.M.Haralick and L.G.Shapiro-Computer and Robot Vision. Volume 1 [9]

Texture of an image is non-figurative and cellular. It is described by the number and types of its (tonal) primitives and the spatial organisation or layout of its (tonal) primitives. The spatial organisation may be random, may have a pairwise dependence of one primitive on a neighbouring primitive, or may have a dependence of $n$ primitives at a time. The dependence may be structural, probabilistic or functional.

\section{- Bernd Jahne-Digital Image Processing [10]}

Patterns which characterise objects are called textures in image processing.

This collection of definitions implies that the texture is formulated by different people depending upon the particular application and suggests that a formal model to describe texture does not exist. This is due to the fact that the texture has been described using intuitive models rather than an analytical formulation. But although a universally accepted definition has not been agreed, two main 
characteristics that are common for all above mentioned definitions can be identified. The first reflects the fact that the texture can be associated with image regions where the neighbouring pixels present a significant variation in intensity levels whereas the second regards the texture as a homogenous property that is repeated at a well defined spatial scale. Early work divided textures into two main types, micro and macro textures. Micro textures are textures with small grey level primitives and the spatial interaction between primitives is constrained to be very local. Macro textures are textures of grey level primitives larger in size than the individual pixel. These primitives have their own identifiable shape properties. An example for micro and macro textures is shown in Figure 1.1. Different techniques and methodologies were developed to describe these textures. A detailed explanation on these techniques is found in [11].

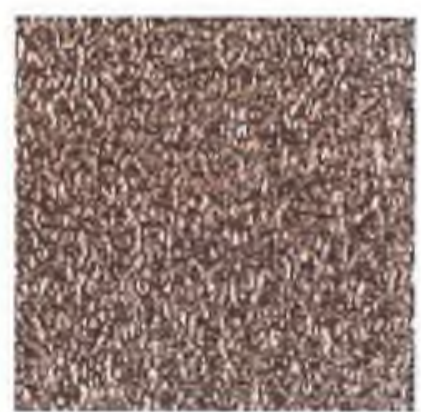

(a)

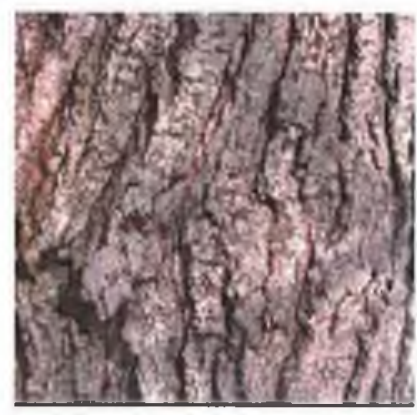

(c)

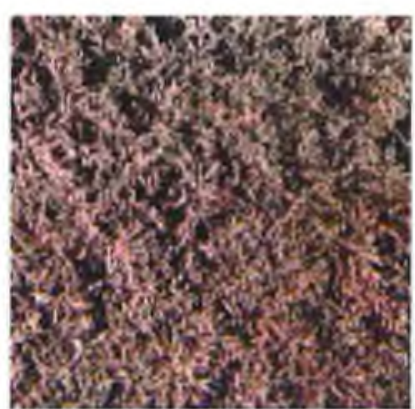

(b)

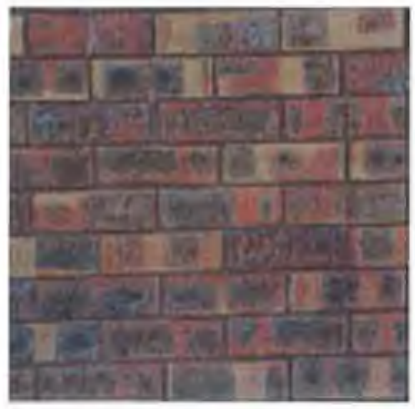

(d)

Figure 1.1: (a), (b) - Micro textures (c), (d) - Macro textures 


\section{Definition of Colour}

Colour is a perceptual result of light in the visible region of the spectrum, having wavelengths from $400 \mathrm{~nm}$ to $700 \mathrm{~nm}$, incident upon the retina. The human retina has three types of colour photoreceptors, hence three components (R - Red, G - Green and B - Blue) are necessary and sufficient to describe a colour. Colour science has mainly concentrated on the research problems, which are based on the tri-chromaticity model of the human visual system. In this model, the idea is that the colour vision is based on three colour sensitive sensors, which have different wavelength sensitivity with each other [12]. Colour vision is inherently tri-chromatic [13].

Colour is an important property and advantageous over intensities. Each image pixel is represented by three bytes corresponding to the values of the red, green and blue components. Hence three values in the place of single intensity value results in more information for image analysis.

\section{Definition of Colour Texture}

Two images with the same colour and different texture patterns or the same texture pattern with different colours are considered as two different colour textured images. Colour texture can be regarded as a pattern described by the relationship between its chromatic and structural distribution [14].

\subsection{Study of Textures}

\section{Texture Synthesis and Analysis}

The three important issues in textures are texture synthesis, texture classification and texture segmentation.

The first issue is on the texture synthesis. The objective of texture synthesis is 


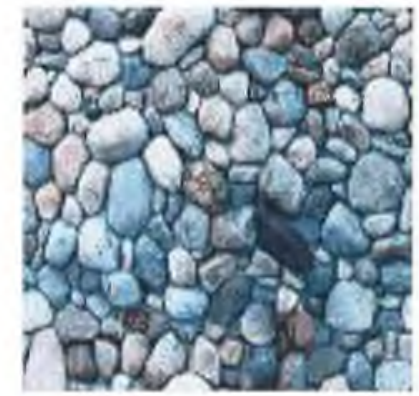

(a)

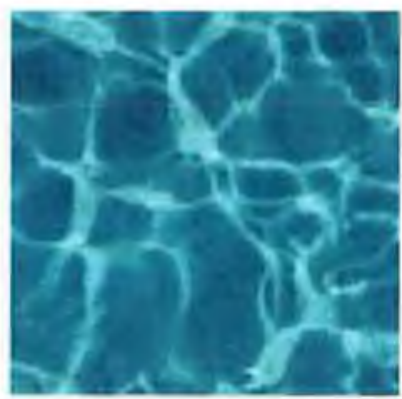

(c)

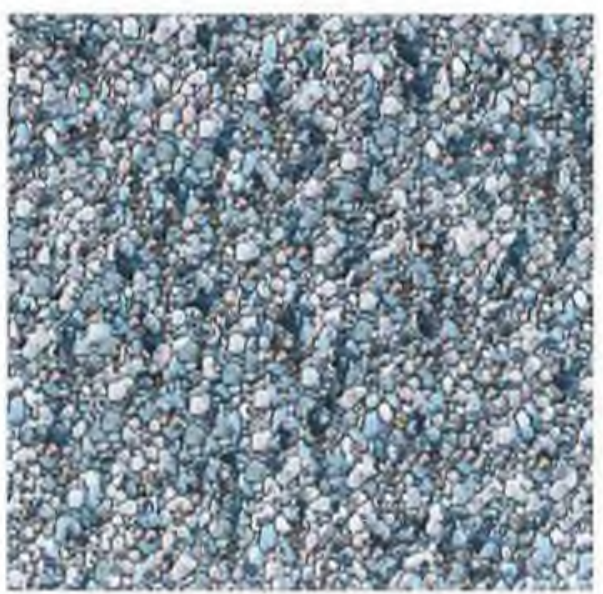

(b)

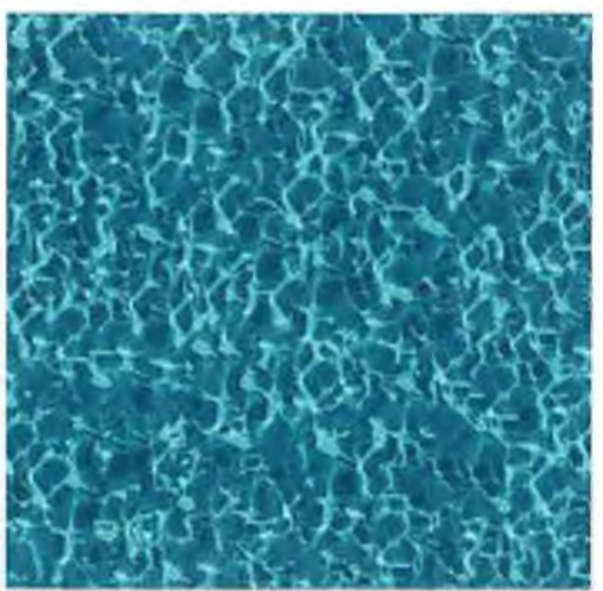

(d)

Figure 1.2: (a), (c) - Input Image (b), (d) - Synthesised Image [15]

to render texture images which are perceptually similar to the observed texture examples. This is often used in image compression applications and in computer graphics to render object surfaces. Texture feature extraction techniques such as Markov Random Field (MRF) and fractal models (see Appendix A) are used to generate synthetically textured images as shown in Figure 1.2.

The second issue is on the texture classification. Figure 1.3 shows different types of textures that are identified as bark, brick, sand and fabric. Identification and 


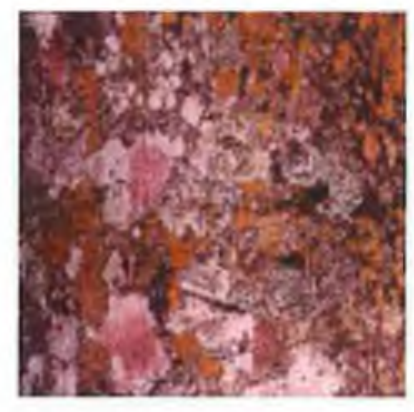

(a)

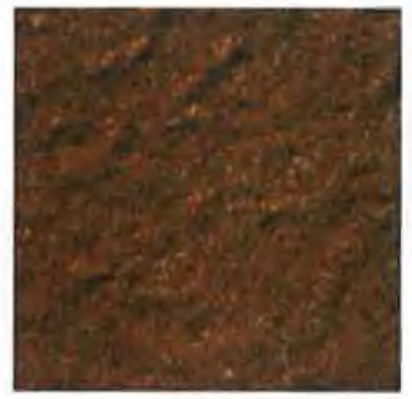

(c)

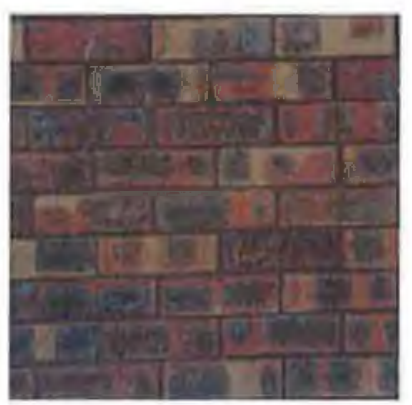

(b)

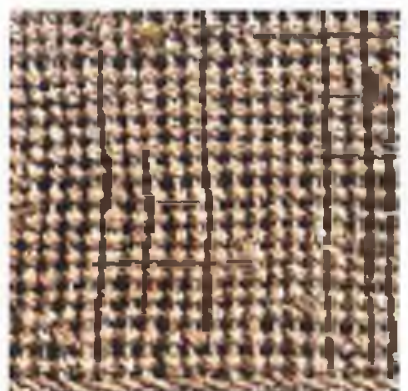

(d)

Figure 1.3: (a) Bark (b) Brick (c) Sand (d) Fabric. Different types of VisTex [27] textures and their identities

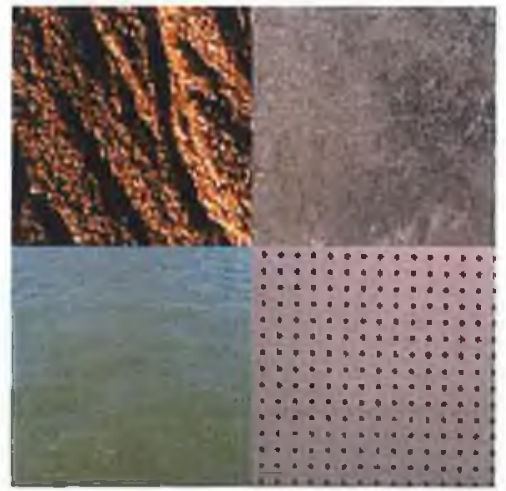

\begin{tabular}{|c|c|}
\hline Sand & Stone \\
\hline Water & Tile \\
\hline
\end{tabular}

Figure 1.4: Different homogeneous textures were labelled in texture classification classification of these types of homogeneous regions is called texture classification. This approach is concerned with the pattern recognition task of textural feature extraction i.e., to extract the texture features using various techniques such as 
co-occurrence, Discrete Cosine Transform (DCT), morphology etc., followed by texture classification. The third issue is on the texture segmentation. Texture segmentation involves partitioning an image into a set of non-overlapping regions whose union forms the entire image. Various methods such as split and merge, region growing are adopted to obtain the segmentation results.

Classification and segmentation processes have closely related objectives. Classification can lead to segmentation, and vice versa. Classification of pixels in an image is another form of component labelling, that can result in segmentation of the objects in the image [6]. Similarly, image segmentation by template matching, as in character recognition, leads to classification or identification of each object. The basic approach for segmentation is to compute the texture features over local neighbourhoods. The neighbourhood is partitioned and clustered using region growing and shrinking methods. Texture segmentation uses a homogeneity measure which is a function of the texture features.

There are two types of texture segmentation, supervised and unsupervised. Supervised segmentation requires a priori information regarding the different textures present in an image. If no assumptions can be made about the type of textures, then unsupervised method is used. In supervised texture segmentation
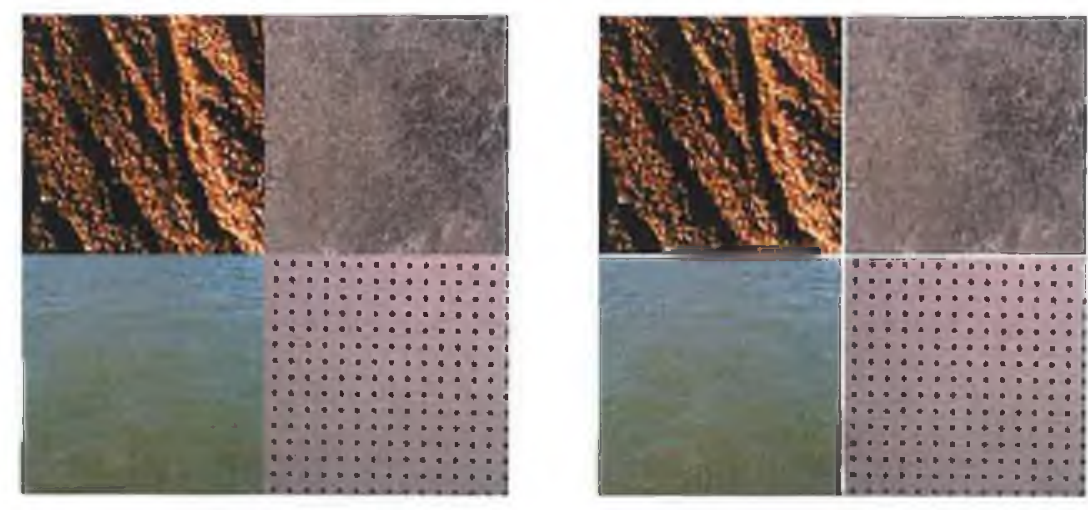

Figure 1.5: Texture segmentation - An example 
each feature vector is assigned to a class as it is generated, whereas in the unsupervised segmentation, the statistical analysis must be performed on the entire distribution of vectors. The goal is to recognise clusters in the distribution and assign the same label to them all. In general, this is a much more complex task to accomplish.

Segmentation of an image entails the division or separation of the image into regions of similar attributes. The most basic attribute for segmentation is the image amplitude-luminance for a monochrome image and the colour components for a colour image [16]. A standard theory of image segmentation does not exist. As a consequence, no single standard method of image segmentation has emerged. There is a collection of methods that have received some degree of popularity. Haralick and Shapiro [9] have established guidelines for a good image segmentation:

- Regions of an image segmentation should be uniform and homogeneous with respect to some characteristic such as grey tone or texture.

- Region interiors should be simple and without many small holes.

- Adjacent regions of a segmentation should have significantly different values with respect to the characteristic on which they are uniform.

- Boundaries of each segment should be simple, not ragged and must be spatially accurate.

Until recently, a limited number of quantitative image segmentation performance metrics has been developed. The methods adopted for colour texture segmentation in this research work are explained in chapter 4. 


\section{Texture Description Methods}

Tuceryan et al. [2] divided the texture feature extraction techniques used for texture description into four categories as: statistical, geometrical, model based and signal processing.

- Statistical methods analyse the spatial distribution of grey values, by computing local features at each point in the image, and deriving a set of statistics from the distributions of the local features. Based on the performance of the method and the texture information captured, the most widely used statistical methods are co-occurrence features, grey level differences, signed differences and Local Binary Pattern (LBP). Other statistical approaches include the autocorrelation function and the grey level run length method.

- Geometrical methods consider textures composed of texture primitives and the rules based on their spatial organisation. The primitives were extracted by edge detection with a Laplacian-of-Gaussian [2], by adaptive region extraction or by mathematical morphology.

- Model based methods are based on the texture process by constructing a parametric generative model which creates the observed intensity distribution. The model can either be pixel based or region based. Pixel based models view an image as a collection of pixels, whereas region based models regard an image as a set of subpatterns placed according to the given rules. Various types of models can be obtained with different neighbourhoods. Examples for this type of model includes one dimensional time series model, autoregressive and moving average model. Random field models analyse spatial variations in two dimensions. Examples of random field models include MRF model, Gaussian MRF model and Gibbs random field model [17] (see Appendix A).

- Signal processing methods analyse the frequency content of the image. Spa- 
tial domain filters such as Laws' masks, local linear transforms and various masks designed for edge detection are the most direct approach for capturing frequency information. The frequency analysis of the tested image was also evaluated by the methods such as Fourier analysis. Other signal processing methods include Gabor transform and wavelet based methods.

\subsection{Colour Analysis}

Colour image analysis has received increased attention in computer vision during this decade. The availability of powerful computational machines combined with novel techniques developed and the technological performances achieved by colour sensors have imposed the use of colour as a preferable alternative to standard grey level approaches. In addition, colour is an important part of the human visual system. It is an intrinsic attribute of an image and provides more information than the grey level intensity values. A colour space is a method by which colour can be specified, created and visualised. The concept of colour space refers to the cartesian space in which the visual sensation of colour can be uniquely defined by a set of numbers representing chromatic features. Colour representation is based on the classical theory of Thomas Young [2]. He stated that any colour can be reproduced by mixing an appropriate set of three primary colours.

Colour is a perceptual phenomenon related to human response to different wavelengths in the visible electromagnetic spectrum. Colour space is a model which represent colours in terms of intensity values. A colour model is the geometric representation of colours in a three dimensional space. Each colour model has its own characteristic, the characteristics generally used to distinguish one colour from another are brightness, hue and saturation. Hue and saturation taken together are called chromaticity and therefore a colour may be characterised by its 
brightness and chromaticity. Sangwine [18] presented an extensive study on the nature of colour, its electronic representation and the concepts of luminance and chrominance. The fundamental colour space in image processing is RGB, which is an additive colour space. In computer processing of colour images, various colour models are used for different purposes. In practice a more appropriate approach is to utilise a colour space, where the information is represented in a better way for the application. There are a number of colour spaces in use depending on the application involved. Some of the widely used colour spaces are HSI, HSV, YIQ, CIE-XYZ, CIE-LAB, CIE-LUV, etc., [19].

In colour analysis, colour plays two important roles, quantitative and qualitative. In a quantitative role three image features from three different planes are obtained as compared to one image feature from grey scale image. The algorithm is derived from those used in monochromatic image analysis with the colour being the additional information. Colour improves the segmentation without increasing the complexity of the algorithms [20]. In a qualitative role, colour is used to acquire intrinsic physical properties from natural images [20].

\subsection{Issues in Colour Texture Segmentation}

There are some issues in colour texture segmentation such as:

- Segmentation is a computationally intensive task. There is a dependency between the image resolution and the speed of processing. The speed of segmentation also depends on feature extraction from texture and colour planes and the segmentation process. Hence, the speed of processing is an important consideration throughout the development of the colour texture segmentation algorithm in this study. The requirement for segmentation is a computationally simple, effective and efficient technique. This resulted in the selection of simple techniques such as the LBP and the DCT based 
filter approach, that derives texture features from the luminance planes. The extraction of colour using statistical features or unsupervised $k$-means colour clustering proved an efficient way to extract colour features.

- The texture and the colour in natural images are often non-uniform due to the change in scale, orientation or other visual distortions [21]. It is also affected by uneven illumination conditions. This causes problems in colour texture image segmentation. The methods for texture segmentation developed have only occasionally evolved in real-world applications [22]. In general, the analysis of natural images has proved to be extremely hard. Hence the approaches should be computationally cheap and robust against the variations aforesaid.

- Natural images do not have well defined boundaries. As a result, segmentation errors will arise while defining the boundaries between two nonhomogeneous regions. These errors are overcome by applying a segmentation refinement algorithm, pixelwise classification or a boundary refinement algorithm, in this research work.

- The incorporation of colour in colour texture analysis is another issue in colour texture segmentation. Various methods are adopted to incorporate the colour information into texture analysis. The selection of appropriate methods to incorporate the chromatic information into texture analysis plays a vital role for colour texture segmentation.

The widely used methods are as follows:

The three spectral band method This is a typical method that has the advantage of simplicity which extends the standard texture analysis techniques such as co-occurrence, LBP, DCT, Gabor filters, etc., to colour images and the result is obtained in each colour plane individually [23]. 
Interaction between spectral bands This approach uses the spatial interaction between different spectral bands. Panjwani and Healey [24] presented an unsupervised texture segmentation algorithm based on MRF models for colour textures. Their models characterised a texture in terms of spatial interaction between spectral bands.

Information from unichrome features and opponent features This method employs the information from both individual colour channel and crosscorrelation features. Jain and Healey [25] introduced a method based on unichrome features computed from the three spectral bands independently and opponent features that utilised the spatial correlation between spectral bands using Gabor filters. They concluded that the opponent colour features significantly improved the classification accuracy over simply using unichrome features.

Combination of colour and texture information This approach divides the colour signal into luminance and chrominance components, the information from both intensity and colour planes were extracted separately and merged together for colour texture processing. Grey scale algorithms are applied to the intensity plane and the colour information is used as additional information [26]. Drimbarean and Whelan [14] examined the contribution of colour information to the overall classification performance by using this approach.

This research work focusses on the last approach where colour and texture information are processed seperately and the distribution of both the luminance and the chrominance features are used for colour texture segmentation. 


\subsection{Organisation of the Thesis}

The thesis structure is organised as follows:

In chapter 2, the related work from the past literature are reviewed. An extensive introduction to the related research work is presented. The research work on texture analysis, colour analysis, colour texture segmentation and classification and colour texture image retrieval methods are also reviewed.

In chapter 3, a detailed explanation is provided on various techniques used in this research work. The feature extraction techniques, the feature preserving adaptive smoothing, the unsupervised $k$-means clustering, the non-parametric tests used for the colour texture description and the unsupervised texture segmentation method are explained. A boundary refinement algorithm which enhances the segmented result is also described. The techniques are combined into the process applied to images to obtain the final segmented result.

In chapter 4, a detailed description on the methodology used in this study is presented. This chapter explains the three methods developed and adopted for colour texture segmentation and details the different colour texture description for the three methods. Flowcharts illustrate the steps followed in the colour texture model for segmentation.

In chapter 5 , different experiments that are performed to observe the effectiveness and the feasibility of the approach is explained. The performance of the system is evaluated using Receiver Operating Characteristic (ROC) curve analysis. In addition, the explanation about the Vision Texture (VisTex) [27] image database, construction of different colour texture images are also discussed. The results of the detailed experiments from mosaic and natural images are presented. Finally, the system implementation of the algorithm is explained. 
In chapter 6 , the application of the developed colour texture segmentation methodology in ISOS script images, skin cancer images and SPI images are presented. In chapter 7 , concluding remarks on this research work is presented.

Finally, in chapter 8 , the contribution of the thesis and the future extensions of this work are suggested.

\subsection{Summary}

Texture and colour are the two innate characteristics and key factors in image analysis. Various methods such as statistical methods, signal processing methods etc., and techniques such as co-occurrence, grey level differences, signed differences, MRF etc., were developed in texture analysis over the past few decades. Colour images provide more information than grey scale images. Colour analysis finds an increasing attention in the recent years. The recent techniques developed for image analysis imposed the use of colour. In computer vision, there are only a few methods that include colour information in texture analysis. Hence, this research focusses on the development of a model for colour texture segmentation. The objective of this research is to evaluate the colour texture segmentation and to determine the role of colour in colour texture analysis. 


\section{Chapter 2}

\section{Review of Related Work}

\subsection{Introduction}

This chapter presents a literature review on texture, colour and colour texture analysis. The study is related to the range of research work published on texture classification, texture segmentation, colour segmentation, colour texture classification, colour texture segmentation, colour texture image retrieval and some general approaches on texture and colour textures. The survey was based on a representative sample of literature that is available in the above mentioned areas and the advantages of the approaches were also explored.

\subsection{Review of Texture Studies}

\subsubsection{Texture Segmentation}

Unser and Eden [28] described an approach for unsupervised segmentation of texture images. Local linear transforms were employed to extract the local texture properties. Texture energy measures were estimated at the output of a filter bank by means of non-linear transformation followed by an iterative Gaussian smoothing algorithm. This procedure generated a multi-resolution sequence of 
feature planes with a half octave scale progression. A new feature reduction technique was applied. The feature reduction technique is an improvement of Karhunen-Loeve transform. The authors concluded that the method provided efficient texture segmentation. The advantage of the method is that it required no prior knowledge about the textures present in the image.

Jain and Farrokhnia [29] presented a multi-channel filtering based texture segmentation technique that used a bank of Gabor filters to characterise the channels. Texture features were obtained by subjecting each filtered image to a nonlinear transformation and computing the energy in a window around each pixel. A square error clustering algorithm was used to integrate the feature images and to obtain segmentation.

Dunn and Higgins [30] presented an algorithm for designing optimal Gabor filters. The procedure used a decision on theoretical framework, based on modelling a Gabor filter output as a Rician distribution, for designing optimal filters. A multiple filter segmentation scheme was also proposed in order to gain more robust results. The authors determined the efficiency of the method experimentally. They claimed that the method performed better and provided useful Gabor filters for a wide range of texture pairs.

Puzicha et al. [31] proposed and examined non-parametric statistical tests to measure texture similarity. The statistical tests were applied to the coefficients of images filtered by a multi-scale Gabor filter bank. The authors found that the similarity measures were useful for both texture based image retrieval and unsupervised texture segmentation. Experiments were conducted on Brodatz micro textures and a collection of real world images.

Hofmann et al. [32] presented novel approaches to segment textured images. This was followed in four steps: First, a scale space approach for data representation 
based on Gabor filters has been suggested. Secondly, a non-parametric statistical test was followed for texture comparison. Thirdly, an unsupervised texture segmentation was formulated as a pair wise data clustering problem based on dissimilarities between texture blocks with a sparse neighbourhood structure. Finally, they developed a general mathematical framework to apply the optimisation principle of deterministic annealing to arbitrary partitioning problems. Segmentation algorithm was tested and validated on Brodatz textures and real world images. They concluded that their approach constitutes a truly unsupervised method for texture segmentation.

Randen and Husoy [33] designed filters for texture feature extraction. They developed and evaluated several approaches for the design of linear finite impulse response filters with optimised energy separation. A model for the feature mean and the variance was developed and the model was used for filter optimisation. This approach was compared with alternative filter optimisation approaches. The approaches were assessed by supervised segmentation experiments.

Ojala and Pietikainen [21] presented an unsupervised texture segmentation method based on the comparison of feature distributions for measuring the homogeneity of texture image regions and to localise boundaries between regions. Texture information was extracted using LBP and contrast. A region-based algorithm was developed for coarse image segmentation and a pixelwise classification scheme for improving the localisation of region boundaries. The advantage of this method is that it does not require any prior knowledge about the number of textures or regions in the image and this method can be easily generalised to utilise other texture features, multi-scale information, colour features and combinations of multiple features.

Wang and Liu [34] proposed a Multi-resolution Markov Random Field (MRMRF) 
texture segmentation. A novel MRMRF parameter estimation method based on Markov Chain Monte Carlo approach was presented. Experiments were performed on different mosaics of natural textures and the authors found that the method was suitable to segment textured images. The disadvantage of this method was the computational complexity.

\section{Summary}

This section presents a survey on texture segmentation. The review shows that techniques such as Local linear transform, Gabor filter, MRMRF, LBP and Linear finite impulse response filter are used to extract texture features. Nonparametric tests are used to discriminate the various texture regions. Different supervised and unsupervised methods are followed for texture segmentation. The performance evaluation of the methods is based on the application of the developed algorithm to Brodatz images and a large number of natural and artificial texture images. Most of the unsupervised texture segmentation methods require no prior knowledge about the texture present in the image. The DCT filter based approach was selected from this survey and the LBP based unsupervised texture segmentation forms the basis for the research work. 


\begin{tabular}{|c|c|c|c|c|c|c|c|}
\hline Ref. & Segn & \begin{tabular}{|l|} 
Feature \\
Extraction
\end{tabular} & Process & Advantages & Disadvantages & Database & Remarks \\
\hline [28] & UTS & \begin{tabular}{|l|} 
Local \\
Linear \\
Transform
\end{tabular} & \begin{tabular}{|l|} 
Non-lingar \\
transf., \\
Gaussian \\
smoothing \\
Unsuge vised \\
fenture \\
reduction
\end{tabular} & $\begin{array}{l}\text { No prior knowledge on } \\
\text { the texture required }\end{array}$ & $\begin{array}{l}\text { Unsup ervised feature } \\
\text { reduction produced } 45 \\
\text { times wor se results } \\
\text { compared to supervised } \\
\text { featur e reduction }\end{array}$ & Brodatz & $\begin{array}{l}\text { DCT filter } \\
\text { ba sed } \\
\text { appr oach } \\
\text { is selected } \\
\text { as the } \\
\text { texture } \\
\text { feature } \\
\text { extraction } \\
\text { bechninue }\end{array}$ \\
\hline [29] & UTS & $\begin{array}{l}\text { Gahor } \\
\text { Filter }\end{array}$ & $\begin{array}{l}\text { Non-linear } \\
\text { transf., } \\
\text { Square error } \\
\text { clustering } \\
\text { algorithm }\end{array}$ & $\begin{array}{l}\text { Simple procedure, } \\
\text { incorporates spatial } \\
\text { information in the } \\
\text { chustering process }\end{array}$ & $\begin{array}{l}\text { The texture segmentation } \\
\text { techmique is omly applied to } \\
\text { the textured images; the } \\
\text { nter features ar e unable to } \\
\text { discriminate between } \\
\text { nearly uniform regims. It } \\
\text { fails in real world images } \\
\end{array}$ & Brodat & \\
\hline$[30]$ & MFS & $\begin{array}{l}\text { Gabor } \\
\text { Fliter }\end{array}$ & $\begin{array}{l}\text { Modelled as } \\
\text { Rician } \\
\text { probability } \\
\text { ilensity } \\
\text { function }\end{array}$ & $\begin{array}{l}\text { The method is useful as } \\
\text { an analytical tool for } \\
\text { studying the } \\
\text { relationship betw een } \\
\text { texture differenceand } \\
\text { the filter requirements } \\
\text { needed to detect those } \\
\text { differences }\end{array}$ & $\begin{array}{l}\text { The presented } \\
\text { segtigntation results exhibit } \\
\text { errors; the errors are } \\
\text { typically confined to the } \\
\text { vicinity of the texture } \\
\text { boundary. Also the } \\
\text { segmattation results with } \\
\text { only two textureare } \\
\text { presented }\end{array}$ & Brodatz & \\
\hline$[31]$ & $\begin{array}{l}\text { UTS, } \\
\text { IR }\end{array}$ & $\begin{array}{l}\text { Gabor } \\
\text { Filter }\end{array}$ & $\begin{array}{l}\text { Non- } \\
\text { parametric } \\
\text { statistic }\end{array}$ & $\begin{array}{l}\text { The draw back of the } \\
\text { feature based methods, } \\
\text { namely the need to } \\
\text { specify a suitable metric } \\
\text { in parameter space, is } \\
\text { avoidgd }\end{array}$ & $\begin{array}{l}\text { The quality far all } \\
\text { mea sures drastically } \\
\text { deteriorates for smaller } \\
\text { images }\end{array}$ & $\begin{array}{l}\text { Brodatz, } \\
\text { Real } \\
\text { world } \\
\text { images }\end{array}$ & \\
\hline [32] & UTS & $\begin{array}{l}\text { Gabor } \\
\text { Filter }\end{array}$ & $\begin{array}{l}\text { Non- } \\
\text { parametric } \\
\text { test, pair wise } \\
\text { data } \\
\text { clustering }\end{array}$ & $\begin{array}{l}\text { Proximity ba sed } \\
\text { approach has been used } \\
\text { to evaluate proximities } \\
\text { between pairs of sites } \\
\text { which rely on proximity } \\
\text { matrix This introduces } \\
\text { sparseness of the data }\end{array}$ & $\begin{array}{l}\text { Misclassificatims at the } \\
\text { boundary are unavoidable }\end{array}$ & $\begin{array}{l}\text { Brodat, } \\
\text { Ae ial } \\
\text { inages, } \\
\text { SAR } \\
\text { images, } \\
\text { Real } \\
\text { worId } \\
\text { images } \\
\end{array}$ & \\
\hline [33] & STS & FIRF & \begin{tabular}{|l|} 
Quadratic \\
Mon-linearity, \\
Smoothing \\
Filter
\end{tabular} & $\begin{array}{l}\text { Few Filters and features } \\
\text { und hence sirnpler } \\
\text { dassification }\end{array}$ & Reduced edge accuracy & Erodatz & \\
\hline [21] & UTS & LEP/C & \begin{tabular}{|l|} 
G-Statistic, \\
Hie archical \\
Splitting \\
Agglomerativ \\
Merging \\
Pixalwise \\
Classification \\
\end{tabular} & $\begin{array}{l}\text { Method can be easily } \\
\text { generalised, not } \\
\text { sensitive to the selection } \\
\text { of parameter values, } \\
\text { does not requir e any } \\
\text { prior knowledge about } \\
\text { the texture present }\end{array}$ & LRP is variant to rotation & $\begin{array}{l}\text { Mosaic of } \\
\text { Natural } \\
\text { inages }\end{array}$ & $\begin{array}{l}\text { Forms the } \\
\text { ba sis for } \\
\text { this } \\
\text { research }\end{array}$ \\
\hline [34] & UTS & MRMRF & \begin{tabular}{|l|} 
Morkov \\
Chain Monte \\
Carln
\end{tabular} & $\begin{array}{l}\text { The method does not } \\
\text { require training on the } \\
\text { textures }\end{array}$ & Computationally intensive & Brobtz & \\
\hline \multicolumn{8}{|c|}{$\begin{array}{l}\text { UTS-Unsupervised Texture Segmentation; STS-Supervised Texture Segneritation; MFS-Mhltiple Filter } \\
\text { Segmentatiom; IR-Image Retrieval; FIRF- Finite Impulse Resp anse Filter; LEP/C-Local Binary Pattern } \\
\text { /Contrast }\end{array}$} \\
\hline
\end{tabular}

Figure 2.1: Section summary - Texture segmentation 


\subsubsection{Texture Classification}

Wang and He [35] demonstrated the usefulness of texture spectrum method for texture classification. The texture spectrum represents the occurrence of distribution of texture unit, which is the local texture aspect of an image. Four of Brodatz [36] natural images have been classified using the texture spectrum method. The success rate for correct classification was $97 \%$ including the influence of the boundaries of different textures.

Unser [37] described a new approach for the characterisation of texture properties at multiple scales using the wavelet transform. A texture was characterised by a set of channel variances estimated at the output of the corresponding filter bank. Classification experiments with 12 Brodatz textures indicated that the discrete wavelet frame approach was superior to the standard wavelet transform feature extraction technique. Unser claimed that the results suggested that the proposed approach performed better than the co-occurrence, local linear transform and similar single resolution techniques. A detailed comparison of the classification performance of various orthogonal and bi-orthogonal wavelet transform were also presented. A discrete wavelet transform feature extraction technique was incorporated into a simple multi-component texture segmentation algorithm. The advantage of this procedure is the possibility of segmentation using less number of features.

Harwood et al. [38] proposed a new method of texture analysis and classification based on a local center-symmetric covariance analysis, using Kullback discrimination of sample and prototype distributions. They introduced two local center-symmetric auto-correlations with linear and rank-order versions (SAC and SRAC) together with a related covariance measure (SCOV) and variance ratio (SVR). These measures are rotation invariant and greyscale invariant. Comparison of these measures was based on the Laws' center-symmetric masks. The 
classification was based on the nearest neighbourhood classifier using Kullback discrimination. The authors concluded that the covariance measures can be regarded as a generalisation of Laws' measures and the performance was better than Laws' approach.

Ojala et al. [39] evaluated the performance of some texture measures such as grey level difference method, Laws' texture measures, center-symmetric covariance and LBP. A method based on Kullback discrimination of sample and prototype distributions was used for classification. The classification results for single features with 1D feature value distributions and for pairs of complementary features with $2 \mathrm{D}$ distributions were also presented. The authors concluded that the grey level difference method achieved the best overall performance discriminating most of the textures.

Randen and Husoy [40] evaluated the filtering approaches for texture feature extraction by performing a comparative study based on the neural network classifier such as Learning Vector Quantisation (LVQ). Filtering approaches included Laws' masks, ring/wedge filters, dyadic Gabor filter banks, wavelet transforms, wavelet packets and wavelet frames, quadrature mirror filters, DCT, Eigen filters, optimised Gabor filters, linear predictors and optimised finite impulse response filters. The features were computed as the local energy of the filter response. The filtering approaches were compared with non-filtering approaches such as co-occurrence and auto-regressive features. The authors concluded that various filtering approaches yielded different results for different images and none of the approaches had superior performance.

Wang and Liu [41] performed texture classification using Multi-resolution Markov Random Field Models (MRMRF). This model combined filtering theory and MRF models. MRMRF considered both high pass and low pass components. 
Brodatz texture database was used to evaluate the performance of Nearest Linear Combination (NLC), which is a measurement of distance to improve the recognition rate. The authors compared the performance of NLC with Nearest Neighbour (NN) and found better results using NLC. They concluded that MRMRF modelling has more powerful texture discrimination ability than using wavelet energy signatures as features.

Maenpaa et al. [42] used the image set of Randen and Husoy to benchmark the LBP approach. LBP is very simple and powerful in various classification and segmentation problems. To process images containing textures at larger scales a simple multi-scale extension of LBP was proposed. The authors claimed that LBP provided the lowest error rate of all operators in ten of the twelve cases. They also claimed that this impressive result was attributable to the grey scale invariance of the LBP operator.

Ojala et al. [43] presented an approach for grey scale and rotation invariant texture classification with LBP and non-parametric discrimination of sample and prototype distributions. The operator characterised the spatial configuration of local image textures. The experimental results obtained in two problems of rotation invariance, where the classifier trained at one particular rotation angle and the classifiers tested with samples from other rotation angles. The authors claimed that the proposed approach was very robust in terms of grey scale variations, since the operators were by definition invariant against any monotonic transformation of the grey scale.

Pietikainen et al. [44] proposed a method for rotation invariant texture classification using feature distributions. The method employs texture measures such as center-symmetric auto-correlation, LBP and a distribution based classification approach. The performance of the proposed approach was compared to that of 
circular-symmetric auto-regressive random field model with a classification problem involving 15 different Brodatz textures and 7 rotation angles. The authors presented a detailed analysis of the confusion matrices and the rotation angles of misclassified samples and produced several interesting observations about the classification problem and the texture features.

Lahajnar and Kovacic [45] presented a method for rotation-invariant 2D texture classification. Energy-normalised texture features were obtained by multi-scale and multi-channel decomposition using Gabor and Gaussian filters. Rotation invariance was achieved by the Fourier expansion of these features with respect to orientation. Non-parametric feature distributions were used for texture description. Experiments were based on two standard datasets, with the classifier trained on samples of only one rotation and tested for all other rotations. The authors concluded that high recognition rates were obtained using this method.

Maenapaa and Pietikainen [46] presented multi-scale binary patterns for texture analysis. They presented two novel ways of extending the LBP texture analysis operator to multiple scales. The large-scale texture patterns were detected by combining exponentially growing circular neighbourhoods with Gaussian lowpass filtering. Cellular automata were proposed to compactly encode arbitrary large binary neighbourhoods. The authors claimed that the main limitations of LBP such as the inability to cope with a large number of different local neighbourhoods were overcome by the multi-resolution LBP. The testing was based on the classification experiments with natural textures from the Outex [47] database.

Turtinen et al. [48] presented a combined use of LBP features and a selforganising map in texture classification. Highly efficient histogram based texture features were utilised with the unsupervised learning and visualisation capabilities of a self-organising map. A log-likelihood distance measure was used suc- 
cessfully instead of the Euclidean distance normally used with the self-organising map. The performance of the approach was empirically evaluated with two different datasets, texture based visual inspection problem containing four similar paper classes and classification of 24 different natural textures from the Outex database.

\section{Summary}

This section presents a review on texture classification. The classification is based on the extraction of texture features using Texture spectrum, Wavelet transform, Local centre-symmetric covariance analysis, Grey level difference method, LBP, MRMRF, and Gabor filter. The classification is based on Nearest neighbour, KNN, Neural network, Bayes and Minimum distance classification. The performance evaluation of the texture techniques is based on the comparison with other classical texture techniques such as Co-occurrence, Law's mask and Local linear transform. The greyscale invariance of the LBP operator outperformed other methods. Comparative study on filtering approaches show better performance of different approaches for different images. Brodatz textures, Outex textures and some natural and artificial texture images are used for testing. 


\begin{tabular}{|c|c|c|c|c|c|c|}
\hline Ref: & $\begin{array}{l}\text { Feature } \\
\text { Extraction Tech. }\end{array}$ & Process & Advantages & Disadvantages & Database & Remarks \\
\hline [35] & $\begin{array}{l}\text { T extmre } \\
\text { Spectrum }\end{array}$ & $\begin{array}{l}\text { Texture Units, } \\
\text { Minimum } \\
\text { distance } \\
\text { dexision rule }\end{array}$ & $\begin{array}{l}\text { Considers the local } \\
\text { texture aspect of an } \\
\text { image }\end{array}$ & $\begin{array}{l}\text { Textur e Spectrum is } \\
\text { sensitive to the } \\
\text { directional asp ect of } \\
\text { the texture }\end{array}$ & Brodatz & \begin{tabular}{|l|} 
Simple \\
technique \\
which is later \\
modified as \\
LBP \\
\end{tabular} \\
\hline [37] & $\begin{array}{l}\text { Wavelet } \\
\text { Transform, } \\
\text { Co-occurrence, } \\
\text { Local Linear } \\
\text { Transform }\end{array}$ & Bayes Classifie & $\begin{array}{l}\text { Less number of } \\
\text { features are used in } \\
\text { this procedure }\end{array}$ & $\begin{array}{l}\text { For images comp osed } \\
\text { of more than two } \\
\text { textures, the } \\
\text { algorithm requires } \\
\text { more sophisticated, } \\
\text { multi dimensional } \\
\text { decision procedure }\end{array}$ & Brodatz & \begin{tabular}{|l|} 
Wavelet \\
Transform \\
outperform \\
other feature \\
extraction \\
techniques
\end{tabular} \\
\hline [38] & $\begin{array}{l}\text { Local center } \\
\text { symmetric } \\
\text { covariance } \\
\text { analysis, Laws } \\
\text { Mask }\end{array}$ & $\begin{array}{l}\text { Nearest } \\
\text { Neighbour } \\
\text { chscification } \\
\text { using Kullback } \\
\text { discrimination }\end{array}$ & $\begin{array}{l}\text { Invariant under } \\
\text { monotonic } \\
\text { greyscale transf. }\end{array}$ & Large err or rate & 13odatz & $\begin{array}{l}\text { This method is } \\
\text { a } \\
\text { generalisation } \\
\text { of Law's } \\
\text { mask, Local } \\
\text { center } \\
\text { symmetric } \\
\text { covariance } \\
\text { analysis } \\
\text { performed } \\
\text { better than } \\
\text { Law's Mask } \\
\end{array}$ \\
\hline [39] & $\begin{array}{l}\text { Grey level } \\
\text { difference } \\
\text { method } \\
\text { (GLDM), Law's } \\
\text { texture moasure, } \\
\text { Local center } \\
\text { symmetric } \\
\text { covariance } \\
\text { moasure, LBP }\end{array}$ & $\begin{array}{l}\text { Nearest } \\
\text { Neighbour } \\
\text { ch scification } \\
\text { using Kullback } \\
\text { discrimination }\end{array}$ & $\begin{array}{l}\text { The greyscale } \\
\text { invariant LEP and } \\
\text { SRAC mmasires } \\
\text { are powerul in } \\
\text { metal sheet } \\
\text { inspection, use of } \\
\text { pairs of } \\
\text { complementary } \\
\text { moasures generally } \\
\text { improves the } \\
\text { classification } \\
\text { accuracy }\end{array}$ & $\begin{array}{l}\text { Poor discrimination } \\
\text { power in the } \\
\text { variances of the } \\
\text { feature distributions }\end{array}$ & $\begin{array}{l}\text { Brodatz, } \\
\text { Metal } \\
\text { sheet } \\
\text { inspectio } \\
\text { n }\end{array}$ & $\begin{array}{l}\text { GLDM } \\
\text { performed } \\
\text { well, LFP is } \\
\text { equally good. } \\
\text { The method is } \\
\text { rotatiomal } \\
\text { variant which } \\
\text { is undesirable } \\
\text { in certain } \\
\text { applications }\end{array}$ \\
\hline$[40]$ & \begin{tabular}{|l|} 
Law's mask, \\
ring'w edge \\
filters, dya dic \\
Gab or filter, \\
wavelet \\
transfurns, \\
Wavelet packets \\
wavelet frames, \\
quadratic \\
mirror filters \\
DCT, eigen \\
tilters optinised \\
Gabor filters, \\
Linear \\
predicturs, \\
FIRF, Co- \\
occurrence, auto \\
regressive \\
\end{tabular} & $\begin{array}{l}\text { Local energy of } \\
\text { filter, Gaussian } \\
\text { filter, Neural } \\
\text { network } \\
\text { ch scifier }\end{array}$ & $\begin{array}{l}\text { DCT approach has } \\
\text { lower feature } \\
\text { dimensionality } \\
\text { resulting in sinple } \\
\text { procedure }\end{array}$ & $\begin{array}{l}\text { All the filters are } \\
\text { complexin nature } \\
\text { exrept DCT }\end{array}$ & $\begin{array}{l}\text { Brodatz, } \\
\text { VisTex, } \\
\text { MeasTex }\end{array}$ & $\begin{array}{l}\text { This study } \\
\text { resulted in the } \\
\text { selection of } \\
\text { DCT filter } \\
\text { based } \\
\text { appr oach for } \\
\text { this research }\end{array}$ \\
\hline
\end{tabular}

Figure 2.2: Section summary - Texture Classification 


\begin{tabular}{|c|c|c|c|c|c|c|}
\hline Ref. & $\begin{array}{l}\text { Feature } \\
\text { Extraction Tech. }\end{array}$ & Process & Advantages & Disadvantages & Database & Remarks \\
\hline$[41]$ & $\begin{array}{l}\text { MRMRF, } \\
\text { Wavelet energy } \\
\text { sigature }\end{array}$ & \begin{tabular}{|l|} 
Filtering \\
theary, MRF, \\
Nearest \\
Linear \\
combination
\end{tabular} & $\begin{array}{l}\text { MRMRF modelling } \\
\text { has mor epow erful } \\
\text { texture } \\
\text { discrimination } \\
\text { ability than using } \\
\text { wavelet energy } \\
\text { sgnature as features }\end{array}$ & $\begin{array}{l}\text { Computationally } \\
\text { Intensive }\end{array}$ & Brodatz & \begin{tabular}{|l|} 
MRMRF \\
outperform \\
Wavelet \\
energy \\
signature
\end{tabular} \\
\hline$[42]$ & $\begin{array}{l}\text { LEP, Mhulti-scale } \\
\text { LEP }\end{array}$ & $\begin{array}{l}\text { Dissimilarity } \\
\text { between } \\
\text { training and } \\
\text { testing } \\
\text { samples was } \\
\text { calculated } \\
\text { using } 3 \\
\text { different } \\
\text { approaches } \\
\end{array}$ & $\begin{array}{l}\text { The multi-predicate } \\
\text { approach is useful } \\
\text { for segnenting } \\
\text { textur es with scaled } \\
\text { structures as scaling } \\
\text { decrease theamount } \\
\text { of information in an } \\
\text { image }\end{array}$ & $\begin{array}{l}\text { The size of the disc } \\
\text { radius has greater } \\
\text { influence on the } \\
\text { segnentation err or } \\
\text { in determining the } \\
\text { texture boundaries }\end{array}$ & $\begin{array}{l}\text { Brodatz, } \\
\text { VisTex, } \\
\text { MeasTex }\end{array}$ & $\begin{array}{l}\text { LEP } \\
\text { performs } \\
\text { better than } \\
\text { nulti- } \\
\text { predicate } \\
\text { LEP }\end{array}$ \\
\hline$[43]$ & $\begin{array}{l}\text { New rntatimal } \\
\text { invariant LEP }\end{array}$ & $\begin{array}{l}\text { Non- } \\
\text { parametric } \\
\text { ch scification } \\
\text { principle }\end{array}$ & $\begin{array}{l}\text { LEP16/VARI6 } \\
\text { demonstrated the } \\
\text { suitability for } \\
\text { rotational invariant } \\
\text { texture classilication }\end{array}$ & $\begin{array}{l}\text { The new LEP } \\
\text { operator is suitable } \\
\text { for discriminating } \\
\text { textures whe e the } \\
\text { dominant features } \\
\text { appear at a very } \\
\text { large scale }\end{array}$ & Brodatz & \\
\hline [44] & $\begin{array}{l}\text { LEPROT, } \\
\text { Center } \\
\text { symmetric auto } \\
\text { c orrelation, grey } \\
\text { levd difference } \\
\text { method }\end{array}$ & $\begin{array}{l}\text { G-statistic, K } \\
\text { nearest } \\
\text { neighbour } \\
\text { principle }\end{array}$ & $\begin{array}{l}\text { LRPROT with a } \\
\text { greyscale variant } \\
\text { feature provides best } \\
\text { results }\end{array}$ & $\begin{array}{l}\text { LBPROT by itself } \\
\text { fairs vey pourly }\end{array}$ & Brodatz & \begin{tabular}{|l|} 
LEPROT \\
was ignored \\
from \\
selection, for \\
this resear ch
\end{tabular} \\
\hline$[45]$ & $\begin{array}{l}\text { Multi-channed } \\
\text { Gabor filter \& } \\
\text { Gaussian filter }\end{array}$ & \begin{tabular}{|l|} 
Rotation \\
invariance \\
achieved \\
using Fourier \\
expansion, \\
non- \\
parametric \\
test \\
\end{tabular} & $\begin{array}{l}\text { Gab or filter of high } \\
\text { frequency produced } \\
\text { botter results }\end{array}$ & $\begin{array}{l}\text { In case of regular } \\
\text { texture with } \\
\text { relatively fine } \\
\text { granularity, higher } \\
\text { Fourier coeficients } \\
\text { did not improve } \\
\text { classification rates }\end{array}$ & Brodatz & \\
\hline [46] & $\begin{array}{l}\text { Multi-scale } \\
\text { binary patten }\end{array}$ & $\begin{array}{l}\text { Gauscian low } \\
\text { pass filtering, } \\
\text { Celhular } \\
\text { automation }\end{array}$ & $\begin{array}{l}\text { The limitations of } \\
\text { LBP such as the } \\
\text { inability to cope with } \\
\text { a large number of } \\
\text { different local } \\
\text { neighb ourhoods } \\
\text { were overcome by } \\
\text { malti-resolutiom LBP }\end{array}$ & $\begin{array}{l}\text { Computationally } \\
\text { Intensive }\end{array}$ & $\begin{array}{l}\text { Natural } \\
\text { Texture, } \\
\text { OrTex } \\
\text { images }\end{array}$ & $\begin{array}{l}\text { This is the } \\
\text { recent } \\
\text { Extension to } \\
\text { LEP }\end{array}$ \\
\hline$[48]$ & LEP & $\begin{array}{l}\text { SOM, Log } \\
\text { likelihood } \\
\text { distribution }\end{array}$ & $\begin{array}{l}\text { SOM based } \\
\text { classification is faster } \\
\text { than KNN classifier }\end{array}$ & $\begin{array}{l}\text { The statistical } \\
\text { instability of the } \\
\text { fentures and the } \\
\text { numerical loss of } \\
\text { data in the iterative } \\
\text { process affects the } \\
\text { performance of } \\
\text { multi-resolution LBP } \\
\text { with SOM }\end{array}$ & \begin{tabular}{|l|} 
Paper \\
classex \\
Natural \\
textures \\
fr om OuT ex \\
Industrial \\
applications
\end{tabular} & \begin{tabular}{|l|} 
LEP \\
outperform \\
GLDM in \\
paper \\
inspection
\end{tabular} \\
\hline
\end{tabular}

Figure 2.3: Section summary - Texture Classification 


\subsubsection{Related Texture Based Approaches}

Haralick [11] presented an extensive survey on various approaches for textures in image processing literature. These include statistical approaches such as autocorrelation function, optimal transforms, digital transforms, textural edgeness, grey tone co-occurrence, run lengths and auto-regressive models. Haralick concluded that the statistical approaches worked well for microstructures. Structural approaches to texture, based on more complex primitives than grey tone were not widely used. For macro textures, histograms of primitive properties and cooccurrence of primitive properties were used. The author concluded with some structural-statistical generalisation. This applies the statistical techniques to the structural primitives.

Unser [49] proposed local linear transforms for texture measurements. This approach is closely related to filter bank analysis method and gives a statistical justification for the extraction of texture properties by means of convolution operators or local matches. Optimal and sub-optimal linear operators were derived for texture analysis and classification. Unser concluded that the method was robust and performed equally as well as co-occurrence based methods for texture classification.

Siew et al. [50] proposed a method for the assessment of carpet wear. The texture feature extraction techniques were used in the carpet industry for carpet grading and quality control. The texture features were computed from second -order grey level statistics and first order grey level difference statistics. These texture features were used to numerically characterise the appearance of the carpets.

Ohanian and Dubes [51] compared and evaluated the performance of four classes of textural features namely co-occurrence, Gabor filters, MRF, and fractal fea- 
tures. The performance of the technique was assessed by the criterion of classification error rate with a nearest neighbour classifier and leave one out estimation method using forward selection. Two synthetic and two natural textures were studied. The authors concluded that comparatively the co-occurrence features performed better followed by fractal features.

$\mathrm{Ng}$ et al. [52] presented a comparative study on Gabor multi-channel model and local linear transform under the computational framework of multi-channel spatial filtering. Though the texture representation concepts of orthogonal DCT basis masks and Gabor filters are fundamentally different, they demonstrated that the representational structure of both approaches are inherently identical. The two approaches were compared experimentally in terms of their computational efficiency and the representation potential of the extracted texture features. The performance of the approaches is assessed by means of their segmentation results on a set of images from Brodatz textures.

Tuceryan and Jain [2] reviewed various aspects of texture analysis. The various texture feature extraction methods such as geometric, random field, fractal and signal processing methods were presented. The major texture processing problems such as segmentation, classification and shape from textures were also discussed. The application areas of the texture were also summarised.

Manjunath and $\mathrm{Ma}$ [53] focussed on using texture information for browsing and retrieval of large image data. Gabor wavelet feature based texture analysis scheme was proposed and its application to image database was demonstrated. Well-known multi-resolution texture classification algorithms were compared. An adaptive filter selection strategy was suggested to reduce the image processing computations. The authors claimed that Gabor feature is robust and the results were encouraging. 
Rui et al. [54] reported a complete survey on different methods of image retrieval based on texture, colour, shape etc. The survey was carried out on technical achievements in visual feature extraction, multi-dimensional indexing and system design. Research issues were identified and future research directions were also suggested.

Chen et al. [55] evaluated the performance of four filtering methods including Fourier transform, spatial filter, Gabor filter and wavelet transform for texture discrimination. Experiments were performed on both natural textures and synthesised MRF textures. The results illustrated that the wavelet features achieved the same identification rate with the Gabor features.

Pietikainen and Ojala [56] developed a non-parametric approach to texture analysis based on simple spatial operators such as local binary pattern and signed grey level differences. The authors claimed that a very good performance was obtained using different texture classification and segmentation problems. They suggested that the complimentary features based on distributions of local spatial patterns and contrast play important roles in texture discrimination.

Ojala et al. [57] proposed the use of signed grey level differences and its multidimensional distributions for texture description. According to the authors, this approach has advantages compared to their earlier approaches based on grey level co-occurrence matrices or histograms of absolute grey level differences. The advantages include, description of texture in a more compact and efficient form, changes in mean luminance do not affect signed differences compared with absolute differences, and provision of more information about image texture. Performance of the signed differences was evaluated using classification of 32 Brodatz textures. The authors claimed that comparable results were obtained using signed differences approach similar to using Gaussian MRF model and Gabor 
filtering.

Singh and Sharma [58] performed five different texture analysis methods on MeasTex [59] and VisTex [27] benchmarks to identify their recognition ability. The texture feature extraction methods included auto-correlation, co-occurrence matrices, edge frequency, Laws' and primitive length. Linear and Nearest Neighbour classifiers were used for classification. The authors concluded with the order of ranking for different methods. They stated that the co-occurrence matrix features were the best and primitive length features were the worst in texture recognition. In addition, they found both the benchmarks performed equally well.

Singh et al. [60] compared the traditional texture analysis techniques on the MeasTex [59] and VisTex [27] benchmarks to illustrate their relative abilities. The different texture methods include auto-correlation, co-occurrence matrices, edge frequency, Laws' masks, run length, binary stack, texture operators and texture spectrum. The authors claimed that both data binary stack method and texture operator method provided excellent results. Also, the feature selection on a combined set of data improved the overall performance.

Zhang and Tan [61] presented a brief review on invariant texture analysis methods. They addressed the texture analysis approaches whose performances are not affected by translation, rotation, affine and perspective transform. The invariant texture analysis algorithms were classified as: statistical methods, model based methods and structural methods. Each approach was reviewed according to its classification and its merits were discussed. 


\section{Summary}

This section presents a review on various approaches for texture description in image processing literature. This section also presents some of the application areas of the texture techniques. The analysis of the methods is based on Brodatz,

VisTex and MeasTex image database.

\begin{tabular}{|c|c|c|c|c|c|c|}
\hline Ref. & $\begin{array}{l}\text { Feature } \\
\text { Extraction Tech. }\end{array}$ & Process & Advantages & Disadrantages & Database & Remarks \\
\hline [11] & $\begin{array}{l}\text { Auto correlation } \\
\text { function, Optimal } \\
\text { transfer, digital } \\
\text { transfer, textural } \\
\text { edgenes, grey } \\
\text { tone co- } \\
\text { occurrence, run } \\
\text { lengths, auto } \\
\text { regressive }\end{array}$ & $\begin{array}{l}\text { Micro-textures, } \\
\text { Structural \& } \\
\text { Statistical } \\
\text { approaches } \\
\end{array}$ & $\begin{array}{l}\text { For microstructures, } \\
\text { the statistical } \\
\text { approach works well }\end{array}$ & & Brodatz & \begin{tabular}{|l} 
Various \\
texture \\
techniques \\
wer e \\
review ed. \\
This paper \\
is the basis \\
for all \\
texture \\
technique
\end{tabular} \\
\hline$[49]$ & $\begin{array}{l}\text { Local Linear } \\
\text { Transform, Co- } \\
\text { occurrence }\end{array}$ & $\begin{array}{l}\text { Optimal } \\
\text { transform \& sub- } \\
\text { optical transform } \\
\text { for texture } \\
\text { classification }\end{array}$ & $\begin{array}{l}\text { Channel variances } \\
\text { aremore power ful } \\
\text { than correlation } \\
\text { coeficients }\end{array}$ & $\begin{array}{l}\text { Classification errors } \\
\text { generally occur } \\
\text { between textures } \\
\text { that are most } \\
\text { difficult to } \\
\text { differentiate visually }\end{array}$ & Brodatz & $\begin{array}{l}\text { This } \\
\text { papers } \\
\text { follows } \\
\text { DCT } \\
\text { approach }\end{array}$ \\
\hline [50] & $\begin{array}{l}\text { Second arder } \\
\text { grey level } \\
\text { statistics, Grey } \\
\text { level difference } \\
\text { method, Grey } \\
\text { lerel run length } \\
\text { matrices, } \\
\text { Neighbouring } \\
\text { grey level } \\
\text { dependence } \\
\text { statistics }\end{array}$ & $\begin{array}{l}\text { Features were } \\
\text { extracted }\end{array}$ & $\begin{array}{l}\text { Texture analysis } \\
\text { texhnique have } \\
\text { useful applications in } \\
\text { the carpet industry }\end{array}$ & $\begin{array}{l}\text { The lighting } \\
\text { arrangement is very } \\
\text { imp ortant in } \\
\text { highlighting textures } \\
\text { for the carpet } \\
\text { assessment }\end{array}$ & \begin{tabular}{|l|} 
Carpet \\
wear \\
assessment
\end{tabular} & \\
\hline [51] & $\begin{array}{l}\text { Co-occurrence, } \\
\text { Gabor filters, } \\
\text { MRF, Fractal } \\
\text { features }\end{array}$ & $\begin{array}{l}\text { Nearest } \\
\text { neighb our } \\
\text { classifier, leave } \\
\text { ane out } \\
\text { estimation }\end{array}$ & $\begin{array}{l}\text { Co-occurrence } \\
\text { features lack the } \\
\text { theoretical under } \\
\text { pinning of the other } \\
3 \text { classes of features } \\
\text { but performed bette } \\
\text { than others }\end{array}$ & $\begin{array}{l}\text { The performance of } \\
\text { MRF featur es was } \\
\text { poor due to the smal } \\
\text { image size }\end{array}$ & $\begin{array}{l}\text { 2synthetic } \\
\& 2 \\
\text { natural } \\
\text { textures, } \\
\text { Leather } \\
\text { images, } \\
\text { painted } \\
\text { surface } \\
\text { images }\end{array}$ & $\begin{array}{l}\text { Co- } \\
\text { occurr ence } \\
\text { perform } \\
\text { better }\end{array}$ \\
\hline
\end{tabular}

Figure 2.4: Section summary - Related texture based approaches 


\begin{tabular}{|c|c|c|c|c|c|c|}
\hline Ref. & $\begin{array}{l}\text { Fenture } \\
\text { Extraction Tech. }\end{array}$ & Process & Advantages & Disadvantages & Database & Remarks \\
\hline [52] & $\begin{array}{l}\text { Gabor filter, } \\
\text { Local Linear } \\
\text { Trandorm (LLT) }\end{array}$ & $\begin{array}{l}\text { Supervised } \\
\text { Bayessian } \\
\text { classifier }\end{array}$ & $\begin{array}{l}\text { Local Variance DCT } \\
\text { gave high quality } \\
\text { segmentation results } \\
\text { using supervised } \\
\text { Bayessian classifiers }\end{array}$ & $\begin{array}{l}\text { Gabor filters } \\
\text { peforms poorly with } \\
\text { stochastic textures. It } \\
\text { is sensitive to } \\
\text { textur es which have } \\
\text { high concentration of } \\
\text { localised spatial } \\
\text { frequency }\end{array}$ & Brodatz & $\begin{array}{l}\text { This paper } \\
\text { forms the } \\
\text { basis for } \\
\text { the } \\
\text { selection of } \\
\text { LLT }\end{array}$ \\
\hline$[53]$ & $\begin{array}{l}\text { Gabor wayelet } \\
\text { feature }\end{array}$ & $\begin{array}{l}\text { Adaptive filter } \\
\text { selection, Image } \\
\text { retrieval }\end{array}$ & $\begin{array}{l}\text { Rotation \& scale } \\
\text { invariance } \\
\text { classification using } \\
\text { Gabor features are } \\
\text { encouraging } \\
\end{array}$ & $\begin{array}{l}\text { Impr owement to tree } \\
\text { structured wavelet } \\
\text { transf. comes at the } \\
\text { expense of much } \\
\text { larger fgature vector }\end{array}$ & Brodatz & \\
\hline [55] & $\begin{array}{l}\text { Fourier } \\
\text { transform, } \\
\text { Spatial filter, } \\
\text { Wavelet transf. }\end{array}$ & Image retrieval & $\begin{array}{l}\text { Wavelet tran form is } \\
\text { computationally } \\
\text { efficient than other } \\
\text { filters }\end{array}$ & $\begin{array}{l}\text { Gabor filter requires } \\
\text { most CPU time }\end{array}$ & $\begin{array}{l}\text { Natural \& } \\
\text { Synthesise } \\
\text { d MRF } \\
\text { texture }\end{array}$ & \\
\hline [56] & $\begin{array}{l}\text { LEP, Signed grey } \\
\text { level difference }\end{array}$ & $\begin{array}{l}\text { Classification } \\
\text { based on UTSM } \\
\text { log likelihood } \\
\text { measure }\end{array}$ & \begin{tabular}{|l|} 
LBP is \\
monotonically \\
invariant to grey \\
scale transformation
\end{tabular} & $\begin{array}{l}\text { LBP is sensitive to } \\
\text { texture orientation }\end{array}$ & Fradatz & \\
\hline$[57]$ & $\begin{array}{l}\text { LEP, Signed grey } \\
\text { level difference \& } \\
\text { Multi } \\
\text { dimensional } \\
\text { signed grey level } \\
\text { distribution }\end{array}$ & $\begin{array}{l}\text { Classification } \\
\text { based on Log } \\
\text { likelihood } \\
\text { measure }\end{array}$ & \begin{tabular}{|l|} 
Description of the \\
texture in a more \\
compact \& efficient \\
fomm, changes in \\
mean luminance \\
does not effect signed \\
differences, \& \\
provide more \\
information about \\
imagetexture \& \\
powerful
\end{tabular} & \begin{tabular}{|l|} 
Greyscale properties \\
should rencain \\
constant throughout \\
the analysis \\
procedure
\end{tabular} & Brodatz & \\
\hline
\end{tabular}

Figure 2.5: Section summary - Related texture based approaches 


\subsection{Review of Colour Image Segmentation}

Colour image segmentation is a process of extracting one or more connected regions from the image domain satisfying uniformity criterion, which is based on features derived from spectral components. The segmentation processes were improved by using additional knowledge about the objects in the scene such as geometric visual properties [62].

Skarbek and Koschan [62] presented a survey on colour image segmentation. They classified colour image segmentation into four categories:

- pixel based segmentation

- area based segmentation

- edge based segmentation

- physics based segmentation

After an extensive analysis of the available literature on colour image segmentation they concluded that:

- Colour images allow more reliable image segmentation than grey scale images.

- Hue feature is used successfully in many applications such as visual perception, skin tumour feature identification, etc.,

- All segmentation techniques depend on the parameters, constants and threshold values, which are usually fixed on the basis of few experiments.

- Several different colour spaces were employed for image segmentation, however, no advantage of one colour space with regard to other colour spaces was observed. 
Shafarenko et al. [63] presented a histogram based segmentation in a perceptually uniform colour space. This algorithm considered the noise that was inevitably present during the image acquisition. A colour clustering method based on morphological watershed transform was performed on the 3D colour histogram. This method was applied to the perceptually uniform LUV colour space. They addressed two different segmentation procedures. The first segmentation procedure was based on the chromaticity and the second segmentation procedure considered both the chromaticity and the intensity of the image. The authors concluded that the resulting algorithm was highly suitable for automatic colour segmentation.

Lucchese and Mitra [64] presented an algorithm for unsupervised colour image segmentation based on low frequency contents. The low pass filtering of images produced smooth segments and sharpens histograms of colour attributes. The algorithm operates in the cylindrical representation of LUV colour space where the representative colours were found by determining the hue families through histogram thresholding and the main clusters on planes by means of $k$-means clustering. The authors claimed the effectiveness of unsupervised colour image segmentation algorithm by discussing two examples of the practical performance of the algorithm.

Wesolkowski and Fieguth [65] presented a colour image segmentation using a region growing method. The region growing algorithm proposed for colour image segmentation is invariant to shading and highlight effects. This method was based on the vector angle colour similarity measure and the principal component of the covariance matrix. The authors claimed that the method has several advantages over other colour image segmentation algorithms. Firstly, the method was based on the colour vector angle, which is a shading-invariant colour similarity measure. This eliminated the intensity variations in the region growing 
process. Secondly, the spatial information taken into account provided separate regions for slightly different colours. The disadvantage lies in the selection of seed pixels. The effectiveness of the algorithm was demonstrated on artificial and natural images.

Chen and $\mathrm{Lu}$ [66] described a colour image segmentation system consisting of two stages of computation: colour clustering in a colour space and colour region segmentation in the image domain. A fuzzy clustering algorithm iteratively generated colour clusters using a uniquely defined fuzzy membership function and an objective function for clustering optimisation. The region segmentation algorithm merged clusters in the image domain based on colour similarity and spatial adjacency. The colour image segmentation system was implemented and tested on a variety of colour images including satellite images, car and face images. The colour image segmentation system combined the two stages of processes to generate effective colour region segmentation. The efficiency of the algorithm was shown through the computational analysis in both stages of computation. Chen and Lu concluded that the results from the colour image segmentation system were successful in finding structures of colour regions, and was flexible in controlling colour resolution, region size and colour distances.

Cheng et al. [67] presented a colour image segmentation approach based on homogram thresholding and region merging. The homogram thresholding employs both the local and global information. The approach considered the occurrence of the grey levels and the neighbouring homogeneity value among pixels. Fuzzy entropy was used to perform homogram analysis for extracting all major homogenous regions at the first stage and the region merging process was carried out based on colour similarity among these regions to avoid over segmentation. Fuzzy entropy considers the fuzziness of the images based on information theory and fuzzy logic and it was used as a criterion to find threshold values automat- 
ically. The experimental results were compared with the standard histogram based approach and found to be efficient. The proposed approach was tested in both RGB and HSI colour space for comparison.

\section{Summary}

This section presents a review on colour image segmentation. Histogram based segmentation, k-means clustering, Region growing, Fuzzy clustering algorithm, Colour similarity, and Homogram thresholding were the different colour image segmentation methods used. Most of the methods are applied to the perceptually uniform LUV colour space. The effectiveness of the algorithms are demonstrated using artificial and natural images. 


\begin{tabular}{|c|c|c|c|c|c|c|}
\hline Ref. & $\begin{array}{l}\text { Colour } \\
\text { Space }\end{array}$ & Process & Advantages & Disadvantages & Database & Remarks \\
\hline$[63]$ & LUV & $\begin{array}{l}\text { Perceptually } \\
\text { uniform, adaptive } \\
\text { filter, Colour } \\
\text { clustering method } \\
\text { based on } \\
\text { morphological } \\
\text { watershed transform }\end{array}$ & $\begin{array}{l}\text { The algorithm performs } \\
\text { automatic colour } \\
\text { segmentation }\end{array}$ & $\begin{array}{l}\text { LUV data are bound to } \\
\text { contain non-unifonm } \\
\text { noise, which could be } \\
\text { sgnificant at places }\end{array}$ & $\begin{array}{l}\text { Variety of } \\
\text { Ima ges }\end{array}$ & \\
\hline$[64]$ & LUV & $\begin{array}{l}\text { Palletised format, } \\
\text { Low pass filter, } \\
\text { Histogram } \\
\text { thresholding k- } \\
\text { means clustering }\end{array}$ & $\begin{array}{l}\text { Exploits the low } \\
\text { frequency content of } \\
\text { colour }\end{array}$ & $\begin{array}{l}\text { Dhe to shadows, } \\
\text { highlights and } \\
\text { reflections, some area } \\
\text { appear to be over } \\
\text { segnented }\end{array}$ & \begin{tabular}{|l|} 
Two \\
images - a \\
bir d and a \\
fruit inage
\end{tabular} & \\
\hline$[65]$ & RGB & $\begin{array}{l}\text { Vector angle colour } \\
\text { suitability measure, } \\
\text { principal compoment } \\
\text { of the cowariance } \\
\text { matrix, region } \\
\text { gr ouping method }\end{array}$ & $\begin{array}{l}\text { Invariant to shading and } \\
\text { highlight effects, } \\
\text { eliminated the intensity } \\
\text { variations, spa tial } \\
\text { information taken into } \\
\text { account, provided } \\
\text { separate regions for } \\
\text { sightly different colpurs }\end{array}$ & Selection of seed pixals & $\begin{array}{l}\text { Artificial } \\
\text { and } \\
\text { Natural }\end{array}$ & \\
\hline [66] & LUV & $\begin{array}{l}\text { Fuxzy clustering } \\
\text { algorithm, region } \\
\text { segmentation } \\
\text { algorithm }\end{array}$ & $\begin{array}{l}\text { This system finds } \\
\text { structures of colour } \\
\text { regions and is flexible in } \\
\text { controlling colour } \\
\text { resolution, region size } \\
\text { and colour distributions }\end{array}$ & $\begin{array}{l}\text { The result depends on } \\
\text { the cluster radius }\end{array}$ & \begin{tabular}{|l|} 
Sa tellite \\
images, car \\
and face \\
images
\end{tabular} & \\
\hline$[67]$ & \begin{tabular}{|l} 
RGB, \\
HSI
\end{tabular} & $\begin{array}{l}\text { Homogram } \\
\text { thresholding region } \\
\text { merging }\end{array}$ & $\begin{array}{l}\text { For a colour image with } \\
\text { high saturation, } \\
\text { segmentation using HSI } \\
\text { can generate good results }\end{array}$ & $\begin{array}{l}\text { In HSI, the non- } \\
\text { removable singularity of } \\
\text { hue may create spurious } \\
\text { modes in the } \\
\text { distribution of values } \\
\text { resulting from the non- } \\
\text { linear transf. which } \\
\text { makes the homogram of } \\
\text { hue value urreliablefor } \\
\text { segmentation }\end{array}$ & $\begin{array}{l}\text { Variety of } \\
\text { images } \\
\text { including } \\
\text { girl, } \\
\text { buttefly, } \\
\text { frog, \& } \\
\text { jellybean }\end{array}$ & \\
\hline
\end{tabular}

Figure 2.6: Section summary - Colour segmentation 


\subsection{Review of Colour Texture Research}

\subsubsection{Colour Texture Segmentation}

Panjwani and Healey [24] presented a colour texture segmentation method based on spatial interaction within each colour plane and interaction between different colour planes. The unsupervised segmentation algorithm presented by them used MRF models for colour texture segmentation. The algorithm consisted of two phases, a region splitting phase and an agglomerative clustering phase. This procedure demonstrated the use of cross plane interaction parameters, that improved the segmentation performance. The authors claimed that the disadvantage of this algorithm was the large number of coefficients in the colour random field model.

Jolly and Gupta [68] described a new algorithm which used maximum likelihood classification combined with a certainty based fusion criterion. The algorithm computed likelihoods independently in each feature space. Segmentation was obtained by evaluating the certainty with each classifier. This algorithm was designed to update old maps of aerial images and to assist a human operator. The algorithm was tested on real outdoor colour scenes and aerial images. The advantage of this technique was that it was not restricted to multi-dimensional colour information. According to the authors, the colour information provided a better localisation of the edges while the texture information provided a less noisy classification of individual regions. The results presented were accurate and the localisation of the edges were excellent.

Shafarenko and Petrou [69] presented a method for processing randomly textured colour images. The method was based on the bottom up segmentation algorithm that considered both colour and texture properties of the image. Watershed transform was applied to an LUV gradient. The patches of watershed 
mosaic were merged according to their colour contrast until a termination criterion was met. The algorithm was tested in automatic granite inspection. The authors concluded that the segmentation procedure has been found to be very robust, producing good results on granite images and also on the wide range of other noisy colour images.

Tremeau and Borel [70] presented an algorithm for colour image segmentation based on a series of region growing and region merging processes. The algorithm began with the region growing process, which took colour similarity and spatial proximity into account. The approach combined both local parameters and global parameters to generate a non-partitioned segmentation of the image. The algorithm was tested in RGB colour space and was extended to other colour spaces. The computation of new threshold values for uniform colour spaces to perform region segmentation remained a constraint for the implementation of the algorithm in other colour spaces.

Petrou et al. [1] described an approach for perceptual segmentation of colour image textures. According to human perception a multi-scale smoothing algorithm smoothes the image. Initial segmentation was achieved by applying a clustering algorithm to the image at the coarsest level of smoothing. Using these isolated core clusters 3D colour histograms were formed and used for probabilistic assignment of all other pixels to the core clusters to form larger clusters and categorised the rest of the image. The process of setting up colour histograms and probabilistic reassignment of the pixels was then propagated through finer levels of smoothing until a full segmentation was achieved at the highest level of resolution. The authors claimed that the perceptual segmentation resulted in $98.3 \%$ of correct segmentation.

Lucchese and Mitra [71] proposed a new procedure for unsupervised segmen- 
tation of colour images based on $k$-means clustering in the chromaticity plane. This procedure identifies the clusters in the $2 \mathrm{D}$ plane containing the chromatic information of colour and successively associated them with appropriate clusters in the $1 \mathrm{D}$ space in which luminance were represented. The $k$-means algorithm was used for both these tasks. The authors claimed that the salient feature of the algorithm was that it handled colour images in a palletised format, which allowed for a very low computational time and was therefore suitable for application in real time. The effectiveness of the technique was experimentally tested on several images.

Paschos and Valavanis [72] proposed a visual monitoring system that performed a scene segmentation based on colour and texture information. The proposed colour texture segmentation system processed luminance and chrominance separately. Filtering, smoothing and boundary detection were performed for the luminance process. Chrominance was processed in two stages, histogram multithresholding and region growing. The presented system was used for various applications such as automated underwater surveillance, wetlands monitoring and in geographical information systems.

Chen et al. [73] developed a new method for colour texture segmentation using feature distributions. The proposed method unifies colour and edge features for colour texture segmentation. The distribution of colour and local edge patterns were used to derive a homogeneity measure for colour texture regions. A regionbased algorithm was developed for coarse image segmentation and a pixelwise classification scheme improved the localisation of region boundaries. Various experiments were conducted to verify the effectiveness and the feasibility of the proposed method. The authors concluded that the error rate for collages of real textures were low and the segmented results for natural scenes were visually satisfactory. 


\section{Summary}

This section presents a review on different colour texture image segmentation methods. Different segmentation methods followed different colour and texture description. Spatial interaction within each colour plane, interaction between different colour planes, and separate processing of colour and texture are the different colour texture description methods followed. MRF models with unsupervised texture segmentation method, Watershed transform, k-means clustering, and colour and local edge pattern are the different techniques adopted for colour texture segmentation. Supervised and unsupervised colour texture segmentation methods are followed to obtain the segmented result. The effectiveness and the feasibility of the proposed methods are experimented using different images

\begin{tabular}{|c|c|c|c|c|c|c|c|}
\hline Ref & $\begin{array}{l}\text { Colour } \\
\text { Space }\end{array}$ & $\begin{array}{l}\text { Colour \&Texture } \\
\text { Processing }\end{array}$ & Process & Advantages & Disadrantages & $\begin{array}{l}\text { Databas } \\
\text { e }\end{array}$ & Remarks \\
\hline$[24$ & RGB & $\begin{array}{l}\text { Spatial interaction } \\
\text { within each colour } \\
\text { plane \& interaction } \\
\text { between different } \\
\text { colour planes }\end{array}$ & $\begin{array}{l}\text { MRF, Region } \\
\text { splitting phase, } \\
\text { Aggl mmerative } \\
\text { clustering phase }\end{array}$ & \begin{tabular}{|l|} 
Good \\
segmentation in \\
the boundaries \\
between regions
\end{tabular} & $\begin{array}{l}\text { The large number of } \\
\text { coefficients in the } \\
\text { colour random field } \\
\text { model }\end{array}$ & $\begin{array}{l}\text { Natural } \\
\& \\
\text { Mosaic } \\
\text { images }\end{array}$ & \begin{tabular}{|l|} 
This \\
procedure \\
wra s carried \\
out in 1095 \\
Later, \\
many \\
techniques \\
were \\
developed
\end{tabular} \\
\hline$[68]$ & $\begin{array}{l}\text { HSI, } \\
\text { YUV }\end{array}$ & $\begin{array}{l}\text { Maxinum } \\
\text { likelihoods from } \\
\text { both texture \& } \\
\text { colmur feature space }\end{array}$ & $\begin{array}{l}\text { Certainty with } \\
\text { classifier } \\
\text { segments the } \\
\text { image }\end{array}$ & \begin{tabular}{|l|} 
This techmiqueis \\
not restricted to \\
multi- \\
dimensiomal \\
colour \\
information
\end{tabular} & \begin{tabular}{|l|} 
The inclusion of \\
some neighbourhood \\
information during \\
the segmentation \\
process may induce \\
segmentation noise
\end{tabular} & \begin{tabular}{|l|} 
Mosaic \\
images, \\
Outdoou \\
scene \\
acrial \\
images, \\
Map \\
updatin \\
g
\end{tabular} & \\
\hline
\end{tabular}

Figure 2.7: Section summary - Colour texture segmentation 


\begin{tabular}{|c|c|c|c|c|c|c|c|}
\hline Ref & \begin{tabular}{|l|} 
Colour \\
Space
\end{tabular} & $\begin{array}{l}\text { Colour \&Texture } \\
\text { Processing }\end{array}$ & Process & Advantages & Disa dvantages & \begin{tabular}{|l|} 
Databas \\
e
\end{tabular} & Remarks \\
\hline$[69$ & LUV & $\begin{array}{l}\text { Automatic } \\
\text { segmentation of } \\
\text { texture and colour }\end{array}$ & $\begin{array}{l}\text { Watershed } \\
\text { transform, LUV } \\
\text { gradient, colour } \\
\text { similarity }\end{array}$ & \begin{tabular}{|l|} 
The \\
segmentation \\
c orresponds to \\
human \\
perception of the \\
image
\end{tabular} & $\begin{array}{l}\text { The limitations of } \\
\text { traditional } \\
\text { approaches is that } \\
\text { the segmentation } \\
\text { sought was based on } \\
\text { the topology of the } \\
\text { image rather than its } \\
\text { intensity }\end{array}$ & \begin{tabular}{|l} 
Granite \\
insp ecti \\
am, \\
Noisy \\
colour \\
images
\end{tabular} & \\
\hline [1] & $\begin{array}{l}\text { SCIE } \\
\text { LAB }\end{array}$ & Pereptial & \begin{tabular}{|l|} 
Molti scale \\
smonthing, K- \\
moans clustering \\
algurithm, 3D \\
colour \\
histograms, \\
probabilistic \\
reassignument
\end{tabular} & $\begin{array}{l}\text { The increasing } \\
\text { size of the } \\
\text { blurring masks } \\
\text { was used to } \\
\text { create the multi- } \\
\text { scale tower. This } \\
\text { includes the } \\
\text { pixels from } \\
\text { larger distances }\end{array}$ & $\begin{array}{l}\text { The Gaussian } \\
\text { smoothing proved to } \\
\text { be inc orrect }\end{array}$ & $\begin{array}{l}\text { Ceramic } \\
\text { tile, } \\
\text { Granite } \\
\text { stone }\end{array}$ & \\
\hline$[71$ & LUV & $\begin{array}{l}\text { Chromaticity from } \\
\text { LD \& 2D plane }\end{array}$ & $\begin{array}{l}\text { Pallette colour } \\
\text { matching, } \mathrm{k}- \\
\text { means colour } \\
\text { clustering }\end{array}$ & $\begin{array}{l}\text { Handled images } \\
\text { in a palletised } \\
\text { format which } \\
\text { allows very low } \\
\text { computational } \\
\text { time }\end{array}$ & $\begin{array}{l}\text { The proposed } \\
\text { algorithm deceived } \\
\text { in corresp ondence of } \\
\text { surfacepatches } \\
\text { affected by } \\
\text { highlighting, mutual } \\
\text { reflections \& dark } \\
\text { shadows } \\
\end{array}$ & \begin{tabular}{|l|} 
Butterfl \\
y, fruit \\
\& bird \\
images
\end{tabular} & \\
\hline$[72$ & $\begin{array}{l}\mathrm{HSI} \& \\
\mathrm{xyY}\end{array}$ & $\begin{array}{l}\text { Separate processing } \\
\text { of Colour \& Texture }\end{array}$ & $\begin{array}{l}\text { Luminance- } \\
\text { illtering, } \\
\text { smoothing, } \\
\text { boundary } \\
\text { detection, } \\
\text { Chr ominance } \\
\text { histogram multi } \\
\text { threstolding } \\
\text { region growing }\end{array}$ & $\begin{array}{l}\text { HSI \& xyY } \\
\text { colour spaces } \\
\text { have } \\
\text { computational } \\
\text { advantage over } \\
\text { an equivalent } \\
\text { RGB based } 3 \\
\text { chanmel sy stem }\end{array}$ & \begin{tabular}{|l|} 
The hue histograms \\
that are highly \\
concentrated in \\
narrow intervals \\
would make it \\
difficult far the \\
sy stem to achieve \\
rea sonable \\
segmentation results
\end{tabular} & \begin{tabular}{|l|} 
Loss'Ga \\
in in \\
wetland \\
Aerial \\
images \\
of \\
wetland \\
scienes
\end{tabular} & \\
\hline$[73$ & $\begin{array}{l}\text { HSI, } \\
\text { CIElab }\end{array}$ & \begin{tabular}{|l|} 
Unifies Colour \\
Texture features
\end{tabular} & $\begin{array}{l}\text { Colour } \\
\text { quantisation, } \\
\text { Local edge } \\
\text { pattern, } \\
\text { Homogeneity } \\
\text { measure, } \\
\text { unsupervised } \\
\text { segmentation } \\
\text { method }\end{array}$ & $\begin{array}{l}\text { This method does } \\
\text { not require any } \\
\text { prior knowledge } \\
\text { about the } \\
\text { number and } \\
\text { types of textures } \\
\text { ar the mumber of } \\
\text { regions in the } \\
\text { image }\end{array}$ & $\begin{array}{l}\text { This method fails in } \\
\text { textures of varying } \\
\text { scalein the same } \\
\text { image. An additional } \\
\text { difficulty in adjacent } \\
\text { regions that have } \\
\text { similar texture \& } \\
\text { colour, particularly } \\
\text { colour because } \\
\text { colour has a greater } \\
\text { effect than LEP }\end{array}$ & VisTex & \\
\hline
\end{tabular}

Figure 2.8: Section summary - Colour texture segmentation 


\subsubsection{Colour Texture Classification}

Tan and Kittler [74] used eight DCT texture features computed from the intensity image and six colour features derived from the colour histogram for the classification of colour textured images. The spatial attributes were gathered by the local linear transform for texture representation and $3 \mathrm{D}$ colour histogram is used as the model for colour representation. The authors claimed that a classification result of $100 \%$ was possible using the colour texture features in a colour granite classification experiment.

Caelli and Reye [23] developed a unified approach to encode colour, texture and shape in a single spatio-chromatic feature space. Features were extracted using multi-scaled filtering and correlation methods that capture the variations of colour over space. The spatio-chromatic features were used for the classification purpose. Here the spatio chromatic image features captured both first and second order measures and envelopes the image qualities of colour, texture and shape. The authors claimed that the advantage of the method was that it unifies the three image properties in a single feature space whereas the traditional image processing methods treat colour, texture and shape in isolation.

Jain and Healey [25] introduced an image classification method, based on colour textures using unichrome and opponent features computed from Gabor filter output. The unichrome features were computed from three spectral bands independently while the opponent features combine spatial information across different spectral bands at different scales. The proposed algorithm reduced the computational complexity in addition to the improvement of the recognition accuracy using colour. A broadband Gabor filter was implemented instead of narrowly tuned Gabor filter. Using a database of 2560 image regions, the authors claimed that the multi-scale approach using opponent features provided better recognition accuracy than other approaches. 
Van de Wouwer et al. [75] extended multi-scale techniques to colour images. They introduced wavelet energy correlation signatures for image classification and derived the transformation of these signatures upon linear colour space transformations. Wavelet correlation signature is defined as the energies of each colour plane and the cross-correlation between different planes. Experiments were performed in real world RGB colour images from different natural scenes. The recognition rates lie between $97-100 \%$ for all classifiers. They claimed that these wavelet correlation features can be employed for other colour texture analysis tasks such as colour segmentation and the addition of colour information does significantly increase the recognition performance.

Paschos [76] presented a method for colour texture classification using CIE chromaticity diagram of an image and a corresponding set of $2 \mathrm{D}$ and $3 \mathrm{D}$ moments to characterise a given colour texture. Its 2D shape described chromaticity i.e., the chromaticity diagram, along with the associated 2D distribution of chromaticity values. This method achieved a success rate of over $90 \%$ and it was applied to the recognition of granite and marble images. Compared with a Gabor multichannel approach, this filtering approach used a small number of chromaticity moment features and achieved a high classification rate.

Drimbarean and Whelan [14] classified the VisTex colour texture images. Three relevant approaches to grey scale texture analysis, namely the filter based DCT, Gabor filtering, and the co-occurrence approach were extended to colour images. The effect of using different colour spaces and the contribution of colour, texture and colour texture features were investigated. The classification performance utilised neural network classifiers based on learning vector quantisation. Two experiments were carried out for colour texture classification. The first experiment concluded that the DCT features produced the highest classification accuracy, followed by Gabor filter and the co-occurrence approach. The second 
experiment concluded that none of the colour spaces proved superior. The highest classification accuracy was obtained using the YIQ colour space. According to Drimbarean, three band and the texture and pure colour classification results were quite similar. Also the experiments performed illustrated that the use of colour improved the performance of standard grey scale texture analysis techniques.

Pietikainen et al. [77] proposed an approach based on separate processing of complimentary colour and pattern information. The distributions of image colour in RGB and Ohta colour spaces were used as features. Ohta colour space is a linear transformation of the RGB space. Histogram intersection was utilised as a. dissimilarity measure. For the texture description, histograms of LBP patterns were used. A log-likelihood dissimilarity measure was used for classification with LBP distributions. Simple variations of the basic LBP operator and a simple joint colour texture LBP operator were implemented. For comparison, the grey scale Gabor operator used by Manjunath and Ma [53] and the joint colour texture operator based on Gabor filtering used by Jain and Healey [25] were employed. The authors claimed that the colour histograms contain discriminative information about the colour dimension of colour texture. Histograms of LBP's provide discriminative pattern related information. The results shows that colour and texture have complimentary roles. The overall classification accuracy of the LBP operator in a $3 \times 3$ neighbourhood was found to be better than that of the much larger Gabor filters. From various experiments, the authors concluded that it was justifiable to use pattern related information such as LBP in addition to the colour measurements.

Paschos and Petrou [78] presented a new method for colour texture classification using self-relative ratio features extracted from the colour histograms. Combining pairs of bins and computing corresponding count ratios, ratio features 
were created that characterise the given colour texture in an auto-correlative manner. Colour histogram method was used for comparison. According to the authors, classification results of VisTex colour texture images showed that the proposed method was accurate and provided improved performance compared to the histogram intersection method.

\section{Summary}

This section presents a review on colour texture classification. The classification is based on the extraction of texture features from the intensity plane and colour features from the colour planes. Techniques such as DCT, Gabor filter, Wavelet energy correlation signatures, Co-occurrence, and LBP are used to extract the texture features. Colour histogram and CIE chromaticity diagrams are used to extract colour features. Performance evaluation of the proposed methods is carried out on VisTex and various other images.

\begin{tabular}{|c|c|c|c|c|c|c|}
\hline Ref & $\begin{array}{l}\text { Colour } \\
\text { \&T exture } \\
\text { Processing } \\
\end{array}$ & Processing & Advantages & Disadvantages & Database & Remarks \\
\hline [74 & $\begin{array}{l}\text { Separate } \\
\text { Colour \& } \\
\text { Texture } \\
\text { processing }\end{array}$ & $\begin{array}{l}\text { B DCT features, colou } \\
\text { histogram, supervised } \\
\text { Bayesian classification }\end{array}$ & $\begin{array}{l}\text { Working with } 3 \\
\text { ldimensional } \\
\text { histogram reduces } \\
\text { computational } \\
\text { complexity and feature } \\
\text { extraction time }\end{array}$ & $\begin{array}{l}\text { Working with } 3 D \\
\text { colour model is } \\
\text { computationally } \\
\text { exp ensive }\end{array}$ & $\begin{array}{l}\text { Colour } \\
\text { granite } \\
\text { ima ges }\end{array}$ & \\
\hline$[23$ & $\begin{array}{l}\text { Spatial } \\
\text { Chrominanc } \\
\text { e feature }\end{array}$ & $\begin{array}{l}\text { Multi-scaled filtering } \\
\text { carrelation methods, } \\
\text { Least squares } \\
\text { minimum distance } \\
\text { chasifier }\end{array}$ & $\begin{array}{l}3 \text { image properties are } \\
\text { unified in a single } \\
\text { feature space whereas } \\
\text { the traditional image } \\
\text { processing methods } \\
\text { treat colour, texture } \\
\text { shape in isolation }\end{array}$ & $\begin{array}{l}\text { The inclusion of } \\
\text { features with low } \\
\text { redumdancy values } \\
\text { resulted in decreased } \\
\text { \& unstable } \\
\text { performance dueto } \\
\text { the increased } \\
\text { likelihood and the } \\
\text { effective addition of } \\
\text { feature noise to the } \\
\text { chasifier }\end{array}$ & $\begin{array}{l}\text { Synthetic } \\
\text { textures }\end{array}$ & \\
\hline
\end{tabular}

Figure 2.9: Section summary - Colour texture classification 


\begin{tabular}{|c|c|c|c|c|c|c|}
\hline Ref & $\begin{array}{l}\text { Colour } \\
\text { \&T exture } \\
\text { Processing } \\
\end{array}$ & Processing & Advantages & Disadvantages & Database & Remarks \\
\hline$[25]$ & $\begin{array}{l}\text { Unichrome } \\
\text { and } \\
\text { opponent } \\
\text { features }\end{array}$ & Gabor filter & $\begin{array}{l}\text { The proposed } \\
\text { algorithm reduces } \\
\text { computational } \\
\text { complexity in addition } \\
\text { to the improvement of } \\
\text { the recognition } \\
\text { accuracy using colour }\end{array}$ & $\begin{array}{l}\text { Choosing the best } 30 \\
\text { features for } \\
\text { cla scification is } \\
\text { subj extive }\end{array}$ & \begin{tabular}{|l} 
Mosaic \\
Images
\end{tabular} & $\begin{array}{l}\text { Colour improwes } \\
\text { the texture } \\
\text { analysis } \\
\text { techniques }\end{array}$ \\
\hline$[75$ & \begin{tabular}{|l|} 
Each colour \\
phne \& \\
cross \\
corr elation \\
betwreen \\
different \\
phnes \\
\end{tabular} & $\begin{array}{l}\text { Wavelet correlation } \\
\text { signatures, k-NN } \\
\text { cla sifier }\end{array}$ & $\begin{array}{l}\text { The wavelet } \\
\text { correlation feature can } \\
\text { be employed for other } \\
\text { Colour Texture } \\
\text { Analysis tasks such as } \\
\text { Colour Segmentation }\end{array}$ & $\begin{array}{l}\text { The recognition } \\
\text { performance is } \\
\text { colour space } \\
\text { dependant }\end{array}$ & $\begin{array}{l}\text { Real world } \\
\text { colour } \\
\text { images }\end{array}$ & $\begin{array}{l}\text { Addition of } \\
\text { Colour improved } \\
\text { theperformance }\end{array}$ \\
\hline [36] & \begin{tabular}{|l|} 
CIE \\
chrominance \\
diagram
\end{tabular} & $\begin{array}{l}\text { Chromaticity } \\
\text { CIEXYZ, 2D-3D } \\
\text { moments, 2D } \\
\text { distribution of } \\
\text { chromaticity. Gabor } \\
\text { multi-channel, } \\
\text { minimum distance } \\
\text { chassification }\end{array}$ & $\begin{array}{l}\text { The method uses a } \\
\text { small number of } \\
\text { chromaticity moment } \\
\text { features \& achieves } \\
\text { high classification } \\
\text { rates }\end{array}$ & \begin{tabular}{|l|} 
Since the samples \\
are drawn randomly, \\
minor fluctuations in \\
the classification \\
rates may be \\
observed when \\
chssification is \\
repeated several \\
times
\end{tabular} & $\begin{array}{l}\text { Granite \& } \\
\text { Marble } \\
\text { images }\end{array}$ & \\
\hline$[14]$ & \begin{tabular}{|l} 
Separate \\
processing of \\
colour \& \\
texture
\end{tabular} & $\begin{array}{l}\text { DCT, Gabor filter, Co- } \\
\text { occurrence, Neural } \\
\text { network classifiers } \\
\text { based on LVQ }\end{array}$ & $\begin{array}{l}\text { DCT features } \\
\text { performs with high } \\
\text { cla ssification accuracy }\end{array}$ & $\begin{array}{l}\text { Computing features } \\
\text { from colour band } \\
\text { determines a } \\
\text { relatively large } \\
\text { number of features } \\
\text { which might lead to } \\
\text { the saturation of the } \\
\text { cha ssifier }\end{array}$ & VisTex & $\begin{array}{l}\text { This survey } \\
\text { provides the } \\
\text { basis for the } \\
\text { selection of DCT } \\
\text { and the separa te } \\
\text { processing of } \\
\text { colour and } \\
\text { texture }\end{array}$ \\
\hline$[77$ & \begin{tabular}{|l|} 
Separate \\
processing of \\
complement \\
ary colour \\
pattern \\
information
\end{tabular} & \begin{tabular}{|l|} 
Distributions from \\
RGB \& Ohta colour \\
space, histogram \\
intersection, log \\
likelihood dissimilarity \\
measure, Basic LEP, \\
i oint colour LBP, \\
Gabor filter
\end{tabular} & $\begin{array}{l}\text { Multi resolution LEP } \\
\text { provides robust } \\
\text { performance } \\
\text { irrespective of } \\
\text { illumination }\end{array}$ & $\begin{array}{l}\text { LEP operates with } \\
\text { small } \\
\text { neighbourhoods } \\
\text { suffer from changing } \\
\text { shadows }\end{array}$ & OuTex & \\
\hline$[78$ & $\begin{array}{l}\text { Separate } \\
\text { Colour \& } \\
\text { Texture } \\
\text { feetures }\end{array}$ & $\begin{array}{l}\text { Self relative ratio, } \\
\text { Colour histograms, } \\
\text { Histogram intersection }\end{array}$ & $\begin{array}{l}\text { This algorithm } \\
\text { recognises image } \\
\text { samples that are } \\
\text { nearly of the same size } \\
\text { as the original image }\end{array}$ & \begin{tabular}{|l|} 
Histogram \\
intersection method \\
provide poor \\
performance \\
compared to $3 \mathrm{D} \mathrm{xyY}$ \\
colour histogram \\
\end{tabular} & VisTex & \\
\hline
\end{tabular}

Figure 2.10: Section summary - Colour texture classification 


\subsubsection{Colour Texture Image Retrieval}

Smith and Chang [79] presented algorithms for extracting, searching, retrieving visual data from image and video databases using colour and texture. The proposed techniques captured colour and texture automatically and represented in binary feature spaces. The binary feature set is a feature representation and indexing. The binary feature set approach for colour represents colour sets by means of local, arbitrarily shaped image regions. Similarly a binary feature set for texture was derived from spatial frequency energy. The texture set captures local texture content and extracts arbitrarily shaped regions of texture from the image. The authors claimed that the binary feature sets performed efficient indexing and image retrieval. Experiments carried out on 3000 colour images proved the effectiveness of the image retrieval.

Randen and Husoy [80] presented a scheme for colour texture feature extraction in image content search. The scheme used the two chrominance components for colour information and a computationally efficient infinite impulse response quadrature mirror filter bank energy measure of the luminance component for texture information. The colour and texture features were combined and their contribution balanced using a modified Euclidean distribution measure. The effectiveness of the scheme was tested on the problem of labelling and finding regions in images. The authors concluded that the proposed scheme out-performed the Gabor features in both quality and complexity.

Kankanhalli et al. [81] presented a context based image retrieval method based on colour and spatial feature distributions. A colour and spatial clustering algorithm was adopted and the colour and spatial feature similarity of the two images were compared using a similarity measure. The proposed technique was tested on two separate databases consisting of 100 synthetic images and 800 real images. The authors concluded that the image retrieval was very efficient for 
both of the databases.

Iqbal and Aggarwal [82] combined structure, colour and texture for efficient image retrieval. Structure was extracted by the hierarchical perceptual grouping principles. Colour analysis was performed by mapping all pixels in an image into a fixed colour palette. Texture analysis was carried out using a bank of even symmetric Gabor filters. A methodology for performance evaluation was presented on a database of colour images. The authors claimed that combining the structure, colour and texture provided a robust image retrieval system. It was demonstrated that the synergy resulting from the combination of structure, colour and texture was superior to using just colour and texture.

Cheng et al. [83] proposed a new method for colour texture image retrieval using colour and edge features. The proposed method unified colour and edge features and the distributions of these features were used to derive a similarity measure for a pair of textures. A retrieval method based on the similarity measure was proposed to retrieve texture images from a database of colour textures of mosaic and natural images. Various experiments were conducted to determine the effectiveness and practicability of the proposed method. The texture database used for the experiments were collected from VisTex texture image database and Ulead Photo Impact Chinese version 6.0. The proposed feature distributions resisted variations in translation, rotation and scale. The authors claimed that the presented method was more effective than the colour histogram method.

\subsubsection{Colour Texture Applications}

Song et al. [84] presented a new colour clustering scheme based on colour perception for defect detection in random textured images. This method used a hybrid chromatic-structural approach to colour texture representation where structural colour texture features were extracted from various chromatic classes associated 
with the colour image texture. A colour clustering method that followed initial clustering and perceptual merging was combined with a binary blob image analysis to capture the relevant information content of the textures. Finally the defects were identified at two stages, colour inspection and blob defect identification. The authors claimed that the algorithm performed better than some classical statistical textural techniques such as the DCT, Gabor functions or the co-occurrence matrices. The colour space used for perceptual merging was uniform and the algorithm worked well for all colour images. The disadvantage for this approach was that for some images dominated by grey colours, the Euclidean metric in the LUV space does not reflect human colour perception.

Kyllonen et al. [85] described a wood surface inspection method that combines colour percentile features with texture features based on simple spatial operators. The colour percentile features were calculated from cumulative colour channel histograms. The texture features were measured using LBP, co-occurrence and signed grey level difference operators. The proposed method was tested with images from an application environment developed for detecting and recognising defects in parquet slabs. The authors stated that the role of colour information in the defects of the wood material was significant. The error rate was much lower with colour percentile features than with the texture features. The co-occurrence features outperformed signed differences and LBP. The disadvantage of colour percentile features was that they do not describe the dependencies between neighbouring pixels.

\subsubsection{Related Colour Texture Based Approaches}

Mirmehdi and Petrou [86] presented a perceptual smoothing for pattern colour separability. The smoothing corresponds to measurements estimated from human psychophysical experiments. Perceptually derived blurring masks were 
found to obtain better image smoothing for colour images compared with the traditional Gaussian approach.

Puzicha et al. [87] empirically compared nine image dissimilarity measures that were based on distributions of colour and texture features. Quantitative performance evaluation of these dissimilarity measures in classification, image retrieval and segmentation tasks were executed. The authors demonstrated that the selection of measures based on large scale evaluation, substantially improved the quality of classification, image retrieval and segmentation of colour and texture images.

Messer and Kittler [88] presented a region based image database system. Each image was segmented into homogeneous colour and texture regions. The mean colour and texture properties of each region were computed and stored as the index to the image, together with the region size and location. To query the database, the user specifies an object on which the same texture and colour properties were calculated. Using this information a neural network was trained which was then used to search through all the indices in the database.

Maenpaa et al. [26] proposed the use of separation of complementary colour and texture measures for colour texture discrimination. RGB histograms were used as colour features together with the Gabor filtering method and the LBP operator. The important finding was that the opponent colour LBP works better than opponent colour Gabor. The results showed that the colour and texture have complementary roles.

Maneesha et al. [19] investigated the role of colour spaces on texture analysis. Colour moments have been used for characterising colour textures. In addition to colour moments, correlogram features were also utilised. VisTex colour texture image database was used for the experiments. Based on the average performance 
of colour spaces, sorted on the basis of combined feature set, the authors ranked the colour spaces in descending order.

\subsection{Summary}

This chapter presented a survey on textures, colours and colour textures. Based on the review of texture research it was observed that various techniques such as Gabor filters, wavelets, co-occurrence, Laws' masks, MRF, LBP etc., were used for extracting texture features. Different methods were examined for classification and segmentation of textures. Most of the techniques are computationally intensive. Hence, the aim of this study is to identify a simple and efficient technique. This resulted in the selection of the LBP technique with the unsupervised texture segmentation method and the filter based DCT approach. The techniques are explained in detail in chapter 3 . This research work proposes different methods for applying these techniques to colour texture segmentation.

Based on previous research of colour analysis it is evident that most methods for colour analysis are based on colour segmentation. Colour moments, colour histograms and colour clustering are widely used in colour segmentation approaches. Colour moments are simple and straightforward mechanisms to describe colours. Colour clustering categorises the objects appropriately based on the properties. These colour techniques were employed in this research work.

There is no explicit model accepted by researchers in computer vision for colour texture segmentation. The quantification of colour and texture in colour texture segmentation is essential to determine the role of colour and texture. The amalgamation of colour and texture for colour texture segmentation and a framework for colour texture segmentation were unresolved and hence the aim of this research work was concentrated on this field of research. It is observed from the 
literature that the methods based on colour texture analysis use different processing of colour and texture features. Some of these are: single spectral band method, cross correlation between different bands and separate processing of colour and texture features. Separate processing of colour and texture features as suggested by Drimbarean et al. [14] is promising. This method of combining colour and textures was selected for the development of the framework for colour texture segmentation.

A wide range of work had been carried out in image analysis including feature extraction, texture classification, texture segmentation, texture synthesis and colour analysis. In the past, segmentation and classification based approaches used only grey scale information. At present, colour is also considered for image analysis. However, little progress has been made in colour texture segmentation. A model for colour texture segmentation to best distinguish different uniform regions remained deficient and hence the goal of this research work focussed on the creation and development of a framework for colour texture segmentation. The presented literature survey on various texture and colour analysis resulted in the selection of simple techniques such as LBP and a filter based DCT approach for texture feature extraction and statistical colour features and colour clustering for colour feature extraction. In addition to these techniques, a method based on separate processing of texture and colour features was also followed. Furthermore, an unsupervised texture segmentation method was followed for colour texture segmentation. 


\section{Chapter 3}

\section{Image Processing Analysis Techniques}

\subsection{Introduction}

The image processing analysis techniques used for this study are described in this chapter. The techniques explained include the two feature extraction techniques, the feature preserving adaptive smoothing, the unsupervised colour clustering and the statistical features for colour processing. In addition, G-statistic, Modified-Kolmogorov Smirnov, unsupervised texture segmentation method and the boundary refinement are also explained. The texture features are extracted using either LBP or DCT technique. Colour processing was performed using the statistical colour features or by colour clustering. The selection of colour spaces for the colour texture segmentation is also discussed.

\subsection{Feature Extraction Techniques}

The aim of feature extraction is to obtain information about the textures present in the image under consideration. A feature is a quantifiable property of the 
image, and is a function of one or more measurements that quantifies significant characteristic of the object. The feature extraction process produces a set of features that, taken together, comprise the feature vector. This reduced amount of information (compared to the original image) can be used for subsequent classification decisions. It is productive to conceptualise an $n$-dimensional space in which all possible $n$-element feature vectors correspond to a point in feature space [89]. Two feature extraction techniques namely, the LBP and the DCT were employed to extract texture features from the intensity plane.

\subsubsection{Texture Feature Extraction Techniques}

\section{Local Binary Pattern (LBP)}

The LBP is one of the feature extraction techniques that provides the pattern related information. LBP is based on two level version of the texture spectrum method developed by Wang et al. [35] and it supplies knowledge about the spatial structure of local image textures. Ojala et al. [39] developed this technique for greyscale images and carried out the texture classification based on feature distributions of different texture measures and found that the method performed well for Brodatz textures [36]. A textured image can be decomposed into a set of small textural units, called Texture Units (TU). A texture unit is represented by 8 elements, each of which has one possible value $(0,1,2)$ obtained from a neighbourhood of $3 \times 3$ pixels. The neighbourhood is represented by $\mathbf{V}=$ $\left\{V_{0}, V_{1}, V_{2}, \ldots V_{8}\right\}$, which generates $3^{8}$ standard texture units, where $V_{0}$ is the center pixel. The occurrence of distribution of texture units is called the Texture Spectrum. LBP is described with $2^{8}=256$ possible texture units. The texture unit $\mathrm{TU}=\left\{\mathrm{E}_{1}, \mathrm{E}_{2}, \ldots, \mathrm{E}_{8}\right\}$ is obtained by applying the threshold operation using the following rule:

$$
\mathrm{E}_{i}=\left\{\begin{array}{cc}
0 & \mathrm{~V}_{i}<\mathrm{V}_{0} \\
1 & \mathrm{~V}_{i} \geq \mathrm{V}_{0}
\end{array}\right.
$$


where $V_{0}$ is the center pixel.

LBP is represented by the equation,

$$
\mathrm{LBP}=\sum_{i=1}^{8} E_{i} \times 2^{i-1}
$$

Since one texture measure is inadequate to describe the grey scale variations of the local texture, LBP is combined with the contrast of the texture, which is a measure of local variations present in the image. Contrast is obtained as the difference between the average grey levels of pixels with value 1 , and pixels with value 0 contained in the texture unit.

The contrast is represented as,

$$
\text { Contrast }=\text { average }_{V_{i} \geq V_{0}}\left(V_{i}\right)-\text { average }_{V_{i}<V_{0}}\left(V_{i}\right)
$$

The above mentioned method is explained with an example:

\begin{tabular}{|l|l|l|}
\hline 2 & 8 & 6 \\
\hline 7 & 5 & 3 \\
\hline 8 & 1 & 9 \\
\hline
\end{tabular}

(a)

\begin{tabular}{|l|l|l|}
\hline 0 & 1 & 1 \\
\hline 1 & & 0 \\
\hline 1 & 0 & 1 \\
\hline
\end{tabular}

(b)

\begin{tabular}{|c|c|c|}
\hline 1 & 2 & 4 \\
\hline 8 & & 16 \\
\hline 32 & 64 & 128 \\
\hline
\end{tabular}

(c)

\begin{tabular}{|c|c|c|}
\hline 0 & 2 & 4 \\
\hline 8 & & 0 \\
\hline 32 & 0 & 128 \\
\hline
\end{tabular}

(d)

Figure 3.1: Steps in the calculation of LBP and $C$ value

$$
\begin{aligned}
& \mathrm{LBP}=2+4+8+32+128=174 \\
& \mathrm{C}=\frac{8+6+7+8+9}{5}-\frac{2+3+1}{3}=5.6
\end{aligned}
$$

The original $3 \times 3$ neighbourhood as shown in Figure 3.1(a), was thresholded by the value of the center pixel to obtain Figure 3.1(b). The value of the pixels in the thresholded neighbourhood were multiplied by the weights given to the corresponding pixels as shown in Figure 3.1(c) to obtain the texture unit Figure 3.1(d). The value of the eight pixels were added to get the LBP value, equation (3.3), of the texture unit. For a better processing of the contrast values, 
the contrast was quantised to 8 bins. Choosing an appropriate quantisation level is an important task, as a lower quantisation level will lack resolution and result in loss of textural information. This resulted in little discriminative information supplied to the process. If the value of the bin is too large, then the histograms are sparse and unstable. Hence the contrast was quantised to 8 bins according to the suggestions made by Ojala et al. [21]. LBP is invariant to any monotonic greyscale transformation. This technique is computationally simple and efficient. Since LBP is variant to rotation, a rotation invariant LBP approach was developed by Pietikainen et al.[44], and other extensions of LBP technique were developed [22], that were beyond the scope of this research work.

\section{Discrete Cosine Transform (DCT)}

The 2D based DCT filtering approach is another feature extraction technique adopted in this work. DCT filtering approach was first suggested by $\mathrm{Ng}$ et al. [52]. Later, Randen and Husoy [40] evaluated this technique by performing a comparative study of texture analysis based on the filtering approaches and obtained improved results using the DCT filtering approach. The DCT filtering approach was applied by using fast separable kernels i.e., row convolution followed by column convolution. A $N \times 1 \mathrm{DCT}$ basis vector is given as follows,

$$
u_{k}= \begin{cases}\frac{1}{\sqrt{N}} & k=0 \\ \sqrt{\frac{2}{N}} \cos \frac{\pi(2 l+1) k}{2 N} & 1 \leq k \leq N-1, \quad 0 \leq l \leq N-1\end{cases}
$$

A set of nine $3 \times 3$ DCT masks are generated from three 1D DCT basis vectors. The basis vectors $u_{k}$ defined in the above equation for $\mathrm{N}=3$ are $u_{0}=\{1,1,1\}^{T}$, $u_{1}=\{1,0,-1\}^{T}, u_{2}=\{1,-2,1\}^{T}$ respectively. A total of nine DCT masks can be produced from these three vectors and they are orthogonal. The 1D filter masks were applied to each row using the outer product and then the transpose of the resulting coefficients were applied to each of the columns yielding nine independent 2D DCT masks. The image was decomposed into linearly 
independent vectors. The distribution of the feature vectors was used to describe the textures. DCT masks are separable in $2 \mathrm{D}$ because they were implemented as a sequence of two 1D filters applied in a cascaded way one after the other [90]. Any 2D $N \times N$ separable mask can be implemented as a cascade of two $1 \mathrm{D}$ masks of size $\mathrm{N}$. This implementation replaces $N^{2}$ multiplications and additions per pixel by $2 \mathrm{~N}$ operations per pixel. DCT is widely used in image coding applications.

\subsubsection{Colour Feature Extraction Techniques}

\section{Statistical Features}

Colour is an effective visual cue. Statistical features such as mean, standard deviation, energy and entropy were computed from the chrominance planes for Method-II (see chapter 4). The colour features are defined as follows:

$$
\bar{x}=\text { Mean }=\frac{1}{N} \sum P(i, j)
$$

The mean is related to the hue.

$$
\text { Std.dev }=\sqrt{\frac{1}{N} \sum(P(i, j)-\bar{x})^{2}}
$$

Any parameter that describes the peak of the distribution around the hue can be used to measure the saturation level. The square root of the variance i.e., standard deviation, is one such measure of deviation and can be derived from moment analysis.

$$
\text { Energy }=\frac{1}{N} \sum P(i, j)^{2}
$$

Energy measures the homogeneity of the colour planes and entropy measures the complexity of the colour planes.

$$
\text { Entropy }=\frac{1}{N} \sum P(i, j) \log P(i, j)
$$


where $P(i, j)$ represents the pixel value at position $i, j$. To make a meaningful use of the parameter values and to obtain a good discriminative power of the colour features, the features were quantised. The colour planes were quantised by means of a simple colour quantisation technique. The quantisation level was selected based on the testing. The features such as mean, standard deviation, energy and entropy were derived from the chrominance planes and the effect of the features in the segmented results were investigated.

\section{$k$-means Clustering}

Clustering analysis is the process of grouping a finite set of objects into subsets. The objects are organised into an efficient representation that characterise the population being sampled. This study uses the unsupervised clustering technique based on the $k$-means algorithm [91]. The $k$-means algorithm is the simplest and most popular among the iterative clustering algorithms. This technique was adopted in Method-III (see chapter 4) to determine the colour clustered distributions.

Initially the partition can be formed by specifying a set of $k$ seed points. Seed points are the first $k$ patterns chosen randomly from the pattern matrix. A set of $k$ patterns, well separated from each other, were obtained by taking the centroid of the data as the first seed point and selecting successive seed points at a distance away from the seed points already chosen. The initial clustering was formed by assigning each pattern to the nearest seed point which forms $k$ clusters. The centroid of the clusters form the initial cluster centers. This forms the initialisation of $k$ points as the seeds for the centroid of $k$ clusters. In the second step, assign each pattern to the centroid closest to the cluster. Calculate the new cluster centers as the centroids of the clusters. Repeat the second step until all the points have their cluster memberships defined. 


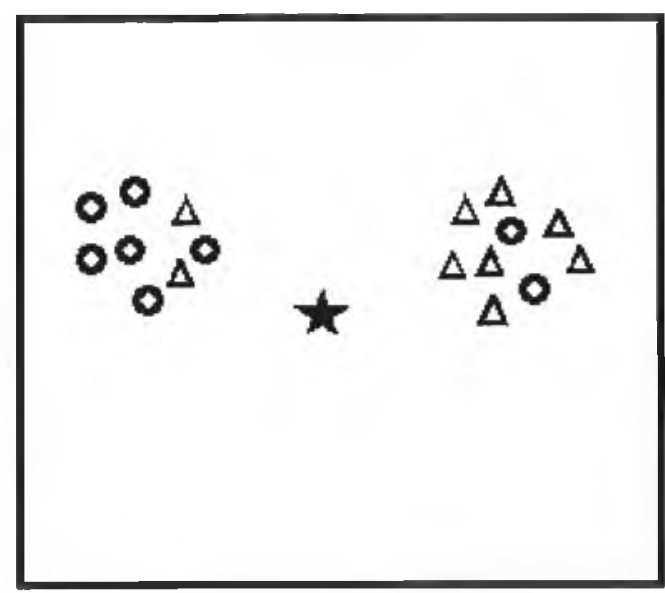

(a)

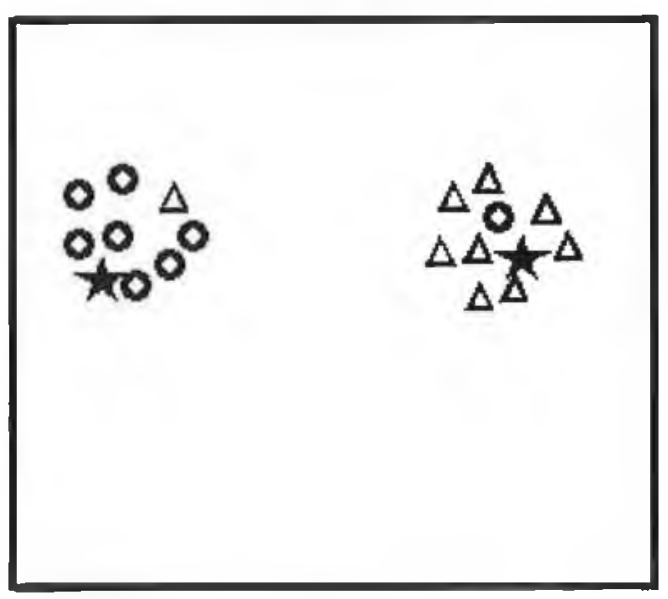

(c)

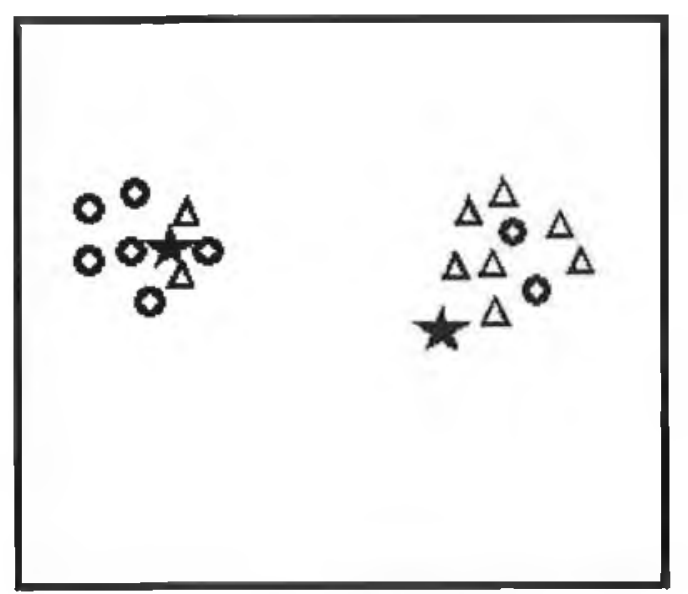

(b)

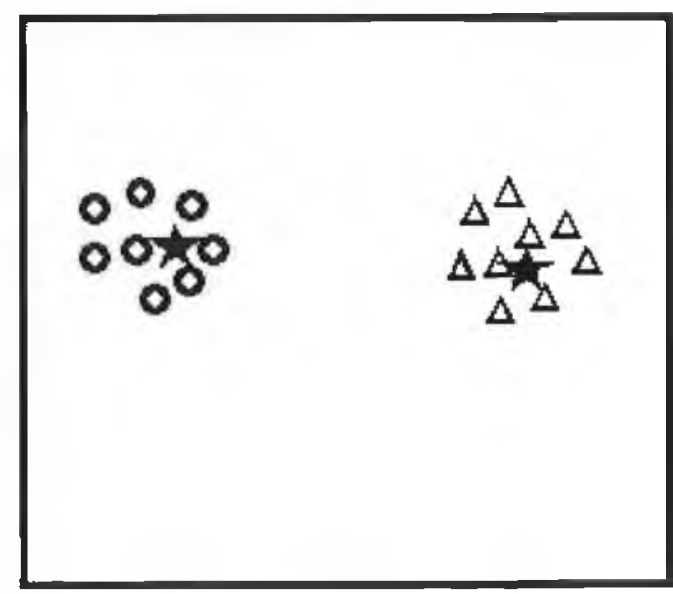

(d)

$\downarrow-$ Cluster center $\quad 0$ - Cluster $1 \quad \Delta$ - Cluster 2

Figure 3.2: k-means clustering method. (a) represents the original image with the different patterns and the centroid of the image data. (b) illustrates the cluster membership after the first iteration. (c) shows the cluster membership after the second iteration. (d) represents the final clustered image.

The number of clusters is the input parameter for the $k$-means algorithm and the value of this parameter is dependent on the complexity of the image. De- 
termination of the optimal number of clusters would be a very difficult task as this parameter is image dependent. In order to address this issue, the number of clusters was set to a value of 10, i.e., sufficiently large to ensure that all important regions in the image with similar colour characteristics are clustered. The proper way to alleviate this is to experiment with different values for $k$. In principle, the best value exhibits the smallest intra-cluster distances and largest inter-cluster distances. As a follow up to $k$-means clustering, the refinement of the initial clusters was based on the merging of clusters that has similar properties. In this regard, two adjacent clusters are merged if the difference between their center values is less than a preset threshold value. This process is iterative and is repeated until no more merges occur. This process allows the algorithm to recover poor initial partitions and select a suitable number of clusters. A detailed explanation of different algorithms for clustering data can be found in [92]. Figure 3.2 shows an example of the $k$-means clustering adopted. In addition to the $k$-means process to reduce the noise and to increase the cluster continuity, before the application of the $k$-means algorithm, the input image was subjected to adaptive smoothing (see section 3.4).

\subsection{Selection of Colour Space}

To process colour images, the first task is to select a suitable quantitative representation of colour i.e., to select a suitable colour space. A number of colour space comparisons were presented in the literature as stated in chapter 2. Different colour spaces have different advantages in representing colour, therefore it is necessary to select the colour space according to the requirement of the task. Hence, it is an important factor to choose an appropriate colour space for image segmentation.

In this research, RGB, HSI, YIQ, CIE-XYZ, CIE-LAB and CIE-LUV colour 
spaces were used in the three spectral band segmentation method. To serve as a prelude, the performance of different colour planes were tested using this method. The segmentation methods based on the unified approach of texture and colour processing focussed on RGB, HSI and YIQ colour spaces, although the method is applicable to any colour space. In order to extract features from colour and intensity plane separately HSI and YIQ colour spaces were selected. Drimbarean [14] et al. comparatively studied the performance of different colour spaces and found that YIQ space performed better than other colour spaces. In addition to this, both YIQ and HSI colour spaces emulate the human visual system. Hence YIQ and HSI colour space was chosen for this study. In this study, the intensity information was extracted from I and Y planes in HSI and YIQ spaces respectively. Similarly, the colour information was extracted from the chrominance planes. In order to compare with the basic standard colour spaces the method adopted for segmentation was also applied to RGB colour space. Brief explanations about the colour spaces are presented in the Appendix B.

\subsection{Adaptive Smoothing}

Various smoothing techniques are widely considered for different purposes in computer vision. In general, smoothing algorithms are classified into two categories, linear and nonlinear smoothing. In linear smoothing, local operators are uniformly applied to an image to form the output intensity of a pixel from a weighted summation of input intensities of its neighbouring pixels [93]. The main disadvantage of linear smoothing is that the boundary between different regions are blurred after smoothing. On the other hand, the nonlinear smoothing preserves important features and also removes noise.

Adaptive smoothing is a nonlinear smoothing which adapts pixel intensities to 
the local attributes of an image on the basis of discontinuity measures. The feature preserving adaptive smoothing algorithm proposed by Chen [93] was adopted, where the local and contextual discontinuity measures were jointly used. To measure local discontinuities, four detectors along four directions are defined as

$$
\begin{aligned}
& E_{H_{x y}}=\left|I_{x+1, y}-I_{x-1, y}\right| \\
& E_{V_{x y}}=\left|I_{x, y+1}-I_{x, y-1}\right| \\
& E_{C_{x y}}=\left|I_{x+1, y+1}-I_{x-1, y-1}\right| \\
& E_{D_{x y}}=\left|I_{x+1, y-1}-I_{x-1, y+1}\right|
\end{aligned}
$$

where $I_{x, y}$ is the intensity of pixel $(\mathrm{x}, \mathrm{y})$. The local discontinuity measure based on the four detectors is defined as

$$
E_{x y}=\frac{E_{H_{x y}}+E_{V_{x y}}+E_{C_{x y}}+E_{D_{x y}}}{4}
$$

To measure contextual discontinuities, the spatial variance was employed to form a measure. A contextual neighbourhood $N_{x y}(R)$ associated with pixel $(x, y)$ was defined as

$$
N_{x y}(R)=\{(i, j) / x-R \leq i \leq x+R, y-R \leq j \leq y+R\}
$$

where $R \geq 1$. The mean and spatial variance are calculated as follows :

$$
\begin{aligned}
\mu_{x y}(R) & =\frac{\Sigma_{(i, j) \epsilon N_{x y}(R)} I_{i, j}}{\left|N_{x y}(R)\right|} \\
\sigma_{x y}^{2}(R) & =\frac{\Sigma_{(i, j) \epsilon N_{x y}(R)}\left(I_{i, j}-\mu_{i j}(R)\right)^{2}}{\left|N_{x y}(R)\right|}
\end{aligned}
$$

In addition to this, $\sigma_{x y}^{2}$ was normalised which is represented as,

$$
\tilde{\sigma}_{x y}^{2}(R)=\frac{\sigma_{x y}^{2}(R)-\sigma_{\min }^{2}}{\sigma_{\max }^{2}(R)-\sigma_{\min }^{2}(R)}
$$


where $\sigma_{\max }^{2}(R)$ and $\sigma_{\min }^{2}(R)$ are the maximal and minimal spatial variances across the image. To alleviate the influence of noise and trivial features, a transformation is added into $\tilde{\sigma}_{x y}^{2}(R)$ as

$$
\Phi\left(\tilde{\sigma}_{x y}^{2}(R), \theta_{\sigma}\right)= \begin{cases}0 & \tilde{\sigma}_{x y}^{2}(R)<\theta_{\sigma} \\ \tilde{\sigma}_{x y}^{2}(R) & \tilde{\sigma}_{x y}^{2}(R) \geq \theta_{\sigma}\end{cases}
$$

where $\left(0 \leq \theta_{\sigma} \leq 1\right)$ is a threshold to limit the degree of contextual discontinuities. Consequently, both the contextual and local discontinuity measures are used for the feature preserving adaptive smoothing. This is defined as

$$
I_{x, y}^{(t+1)}=I_{x, y}^{(t)}+\eta_{j x y} \frac{\Sigma_{(i, j) \in N_{x y}}(1) \backslash\{(\tau, y)) \eta_{i j} \gamma_{i j}^{(t)}\left(I_{i, j}^{(t)}-I_{x, y}^{(t)}\right)}{\Sigma_{(i, j) \in N_{x y}(1) \backslash\{(x, y)\}} \eta_{i j} \gamma_{i j}^{(t)}}
$$

where $I_{x y}^{(t)}$ is the intensity of pixel $(x, y)$ at iteration t. $\eta_{i j}$ and $\gamma_{i j}^{(t)}$ are defined as

$$
\begin{aligned}
& \eta_{i j}=\exp \left(-\alpha \Phi\left(\tilde{\sigma}_{i j}^{2}(R), \theta_{\sigma}\right)\right) \\
& \gamma_{i j}^{(t)}=\exp \left(-E_{i j}^{(t)} / S\right)
\end{aligned}
$$

From equation (3.19), $\alpha(\alpha>0)$ is used to determine the extent of feature preservation in terms of contextual discontinuities. The parameter $\alpha$ provides a way to control the smoothing speed and is also used to determine the weights for averaging the pixels associated with important features. A small value of $\alpha$ results in fast smoothing and discontinuity reduction, while a large value leads to a slow smoothing at the pixels with high contextual discontinuities and preserves the important features.

From equation (3.20), $S(S>0)$ is used to determine the preservation extent of local discontinuities. The parameter $S$ determines the magnitude of the edges to be preserved during smoothing. The preservation of the local discontinuities was based on the pixels whose contextual discontinuities were not considered due to the thresholding effect resulted from equation $\Phi\left(\tilde{\sigma}_{x y}^{2}(R), \theta_{\sigma}\right)$. Based on the 
nonlinear transformation or exponential function, $\eta$ encodes the effect of contextual discontinuities and $\gamma$ encodes the effect of local discontinuities. The results shown in chapter 5 corresponds to $\alpha=10$ and $S=10$. The values were selected based on testing on a range of values and as per the effects of $\alpha$ and $S$.

For any pixel $(x, y)$ in the image, $I_{\min } \leq I_{x, y}^{(t+1)} \leq I_{\max }$, where $I_{\max }$ and $I_{\min }$ denote the maximal and minimal intensity across a given image. This reflects the algorithm's convergence. The advantage of this smoothing technique is that the parameters in the given adaptive smoothing algorithm critically examines the smoothing process. The parameters preserves the edges and removes noise. This procedure was used to prevent over segmentation. The effects of the parameters were discussed and a detailed explanation on the adaptive smoothing and the implementation of the algorithm is given in [93].

\subsection{Non-parametric Test}

Non-parametric test statistics provide a sound basis for probabilistic procedures. This test makes no assumptions about the frequency distributions of the variables being assessed. The hypothesis that the two empirical feature distributions have been generated from the same population was tested using the non-parametric test. Colour and texture description vary considerably over an image due to the inherent variation in surface appearance and also due to the changes in illumination, shading etc. Hence, the appearance of the region is best described by the distribution of features, rather than individual feature vector. Histograms are used as non-parametric estimators of empirical feature distributions [87]. The discrimination between the distributions was based on two dissimilarity measures. G-statistic and Modified Kolmogorov-Smirnov are the two non-parametric tests used to test the colour texture distributions. 


\subsubsection{G-statistic}

To compare the feature distributions in Method-I and Method-II (explained in chapter 4), the log-likelihood pseudo metric G-statistic was employed. G-statistic is the dissimilarity measure used by Ojala et al. [21] together with the LBP approach and the unsupervised texture segmentation. Hence, this non-parametric test statistic was followed. In G-statistic, the similarity of the two histograms were measured with a two way test of interaction or heterogeneity. The Gstatistic is defined by the equation:

$$
\begin{aligned}
G= & 2\left\{\left[\sum_{s, m} \sum_{i=1}^{n} f_{i} \log f_{i}\right]-\left[\sum_{s, m}\left(\sum_{i=1}^{n} f_{i}\right) \log \left(\sum_{i=1}^{n} f_{i}\right)\right]\right. \\
& -\left[\sum_{i=1}^{n}\left(\sum_{s, m} f_{i}\right) \log \left(\sum_{s, m} f_{i}\right)\right] \\
& \left.+\left[\left(\sum_{s, m} \sum_{i=1}^{n} f_{i}\right) \log \left(\sum_{s, m} \sum_{i=1}^{n} f_{i}\right)\right]\right\}
\end{aligned}
$$

where $f_{i}$ is the frequency at bin $i$; $s$ and $m$ are the two sample histograms; and $n$ is the number of bins. Higher the value of $G$, lower the probability that the two samples are drawn from the same population. The results obtained using G-statistic using Method-I and Method-II does not produce proper segmentation. The selection of a proper dissimilarity measure substantially improves the quality of the segmentation. Hence, in order to improve the performance of the segmentation, the Modified-Kolmogorov Smirnov was considered as the dissimilarity measure for Method-III (explained in chapter 4).

\subsubsection{Modified-Kolmogorov Smirnov (M-KS)}

The non-parametric test used in Method-III for comparing LBP/C with colour clustered labels or DCT with colour clustered labels was a M-KS statistic. The standard Kolmogorov Smirnov (KS) was modified and used for the implementation. KS is otherwise called as D-statistic or the discrepancy statistic. M-KS tests the data in continuous distribution. The M-KS statistic is defined as the 
sum of the absolute value of the discrepancies between the normalised cumulative distributions.

$$
\begin{aligned}
D(s, m) & =\sum_{i=1}^{n}\left|\widehat{F}_{s}(i)-\widehat{F}_{m}(i)\right| \\
& =\sum_{i=1}^{n}\left|\frac{F_{s}(i)}{n_{s}}-\frac{F_{m}(i)}{n_{m}}\right|
\end{aligned}
$$

where $F_{s}(i)$ and $F_{m}(i)$ represent the sample cumulative distribution functions; $n_{s}$ and $n_{m}$ represents the number of pixels in the sample regions. Since M-KS is normalised, it is advantageous over G-statistic. This method has the advantage in discriminating the feature distributions over standard KS statistic. Similar to KS statistic, M-KS has the desirable property that it is invariant to arbitrary monotonic feature transformations [87].

\subsection{Unsupervised Texture Segmentation Method}

Segmentation is the process of separating mutually exclusive homogeneous regions of interest. There are numerous techniques for the extraction of texture features and various methods for the texture segmentation. Predominantly, the methods were developed for greyscale textures. More recently, attempts were made to develop methods that combine colour and texture features for the segmentation. The unsupervised grey scale texture segmentation method developed by Ojala et al. [21] was used in this research for colour texture segmentation. The unsupervised texture segmentation method involved three steps:

- Hierarchical splitting

- Agglomerative merging

- Pixelwise classification

Hierarchical splitting divides the image into blocks of roughly uniform texture and the agglomerative merging procedure merges similar adjacent regions until a 
stopping rule is satisfied. This results in segmentation of different homogeneous textured regions in the image. Pixelwise classification improves the localisation of the boundaries and leads to an enhanced segmented image [21]. A novel data structure is proposed for the implementation of unsupervised texture segmentation. Data structures are important for the direct representation of image information, to decide a strategy for processing the computationally intensive algorithms on the basis of relatively small quantities of data and to create much easier and efficient image processing [94]. The developed data structure used a quadtree which splits the image and a mergegraph to merge the adjoining regions. The quadtree data structure is a standard hierarchical data structure, but the novelty lies in the use of this structure for the hierarchical splitting. The resulting quadtree from splitting stage was used in the merging process and the creation of mergegraph for agglomerative merging.

Hierarchical Splitting The hierarchical splitting recursively splits the image into four rectangular blocks of varying size. A block was split into 4 subblocks based on a uniformity test. The six pairwise $\mathrm{G}$ values between the $\mathrm{LBP} / \mathrm{C}$ histogram or DCT histogram of the 4 subblocks were calculated. The maximum G value is represented by $G_{\max }$ and the minimum $\mathrm{G}$ value by $G_{\min }$. The uniformity of the region was tested by a decision factor $R=\frac{G_{\max }}{G_{\min }}>X$ where $\mathrm{X}$ is a threshold value. The choice of $\mathrm{X}$ was based on the testing for different values of $\mathrm{X}$. The selection of $\mathrm{X}$ should be small to allow more number of splits. The threshold value $X$ was experimentally set to a value of 0.9 . An error occurs when a block defined by uniform texture is split into four blocks. In that situation the merging procedure can easily compensate for the over split. On the other hand, if several textures were considered as uniform then the error recovery is not possible. If the relation $R>X$ is true, then the block was considered to be inhomogeneous and was further split into four blocks. The procedure of splitting the block was repeated recursively on each subblock until a block size $S_{\min }$ was 


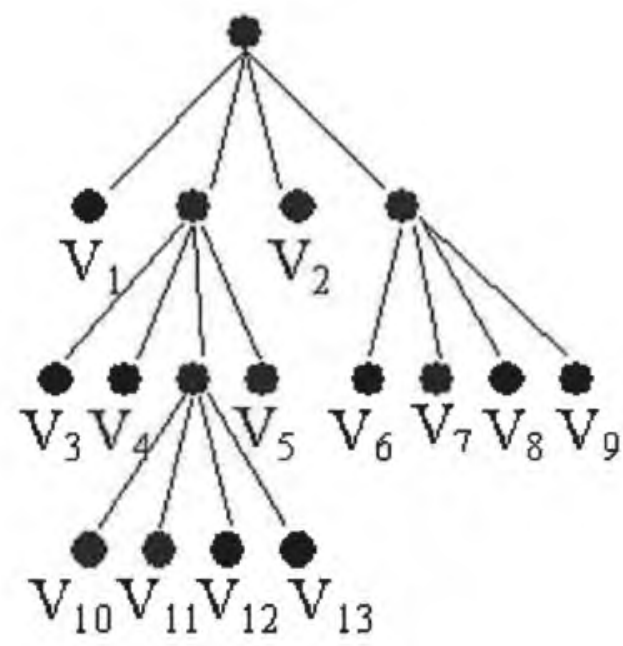

(a)

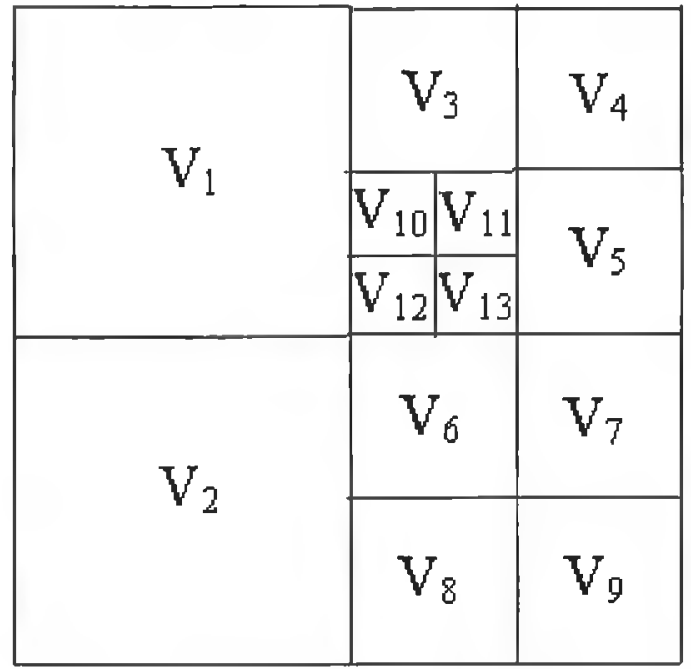

(b)

Figure 3.3: A typical example of the splittree and the corresponding image representation

reached. The value for $S_{\text {min }}$ is set to $32 \times 32$ [21]. Comparable results were obtained with value $16 \times 16$, whereas $8 \times 8$ or $4 \times 4$ are prone to noise. Method-II adopts a similar procedure except for the use of LBP/C + colour features or DCT + colour features. The same procedure was adopted in Method-III as well except that the M-KS value was used instead of $G$ value.

This algorithm was implemented using a quadtree structure. A quadtree structure is represented as a splittree (Figure 3.3(a)) with every parent node having 4 child nodes and the corresponding image representation was shown in Figure 3.3(b). This algorithm generates a splittree by using an iterative procedure. The tree was initialised with one node referring the whole image. The iterative procedure traverses through the leaves of the tree. If the image area corresponding to a particular leaf is inhomogeneous then it is split into 4 child nodes. This iteration stops when there are no more leaves to be split or the image size of an unsplit leaf is $S_{m i n}$. The leaves of the splittree at the end of the iteration proce- 
NodeType Head ;

Head.Image = Image;

// Im age is assigned to Head of Tree

while(1)

\{

// Start Iter ation

int No_of_leaves;

NodeType ${ }^{\star}$ Leaf $=$ GetLeaves(Head,\&No_of_leaves); //Scan through all the leaves

FLAG $=0$;

for $(i=0 ; i<$ No_of_leaves $; i++)$

\{

if(!Homogen eous(Leaves[i].Image))

\{

split(Leafli|)

FLAG $=1$;

了

\}

if(FLAG $==0$ )

break;

\}

"Check for homogeneity

//split the im age of ith leaf into four

//end if

/end for

$/ / F L A G=0$ for Exit Condition

//end while

Figure 3.4: Pseudo code for the splittree approach

dure refers to homogeneous regions in the image. These leaves were denoted as $\mathrm{V}=\left\{\mathrm{V}_{1}, \mathrm{~V}_{2}, \mathrm{~V}_{3}, \ldots, \mathrm{V}_{N_{s}}\right\}$ where each $\mathrm{V}_{i}$ is a leaf and the $i^{\text {th }}$ index is selected arbitrarily and $N_{s}$ is the number of homogenous regions $s$. Also, the union of all $\mathrm{V}_{i}$ equals the whole image, i.e., $\mathbf{I}=\bigcup_{i=1}^{n} \mathrm{~V}_{i}$. Figure 3.4 illustrates the pseudo code for the splittree approach.

Agglomerative Merging An agglomerative merging procedure was applied to the image which has been split into blocks of roughly uniform texture. This procedure merges similar adjacent regions until a stopping rule was satisfied. The pair of adjacent segments which has the smallest Merger Importance (MI) value were merged. MI was calculated from $M I=p \times G$, where $p$ is the smaller number of pixels among the two regions and $G$ is the value of G-statistic between the two regions. After merging, the two respective LBP/C histogram or DCT histograms were summed to be the histogram of the new image region, which updates the segmented image. Then the $\mathrm{G}$ distribution between the new region 
and all adjacent regions were computed. The merging procedure was adopted until the stopping rule was satisfied. $M I R=\frac{M I_{\text {curr }}}{M I_{\text {max }}}>Y$, where $M I_{\text {curr }}$ is the merger importance of the current merge, and $M I_{\max }$ is the largest merger importance of all preceding merges. If the ratio MIR exceeds the threshold value $\mathrm{Y}$, the merging procedure was halted. The threshold $\mathrm{Y}$ was found experimentally. The algorithm was applied to numerous images and the MIR values were examined. Depending on the images, the threshold value ranges between 1.2 and 1.4 . Theoretically, in the initial merges, the adjacent regions with identical LBP/C or DCT histograms, have a zero MI value which will lead to the termination of merging prematurely. Hence for the first few merges, the stopping rule was not verified [21]. Method-II used colour features together with LBP/C or DCT histograms.

In Method-III, the pair of adjacent segments were merged according to the minimum MI value. $\mathrm{MI}$ is calculated as $M I=w_{1} \times M K S_{1}+w_{2} \times M K S_{2}$, where $w_{1}$ and $w_{2}$ represent the weights which were calculated according to the distribution of the colour clustered labels and the detailed explanation of the weights and the colour texture description are explained in chapter 4 . If two regions were merged then their corresponding texture histograms were summed and the colour histograms were summed to be the histograms of the new region and the segmented image is updated. This method followed a simple stopping rule, $\operatorname{MinMI}>Y$, where MinMI represents the minimum merger importance value. If this is greater than a threshold value then the merging procedure is halted. The agglomerative merging procedure resulted in a blocky segmented images. A refinement of the boundaries are performed using the pixelwise classification. The implementation of the agglomerative merging was performed by creating a mergegraph. A mergegraph is a triplet $G=(\mathbf{V}, A, E)$ where $\mathbf{V}$ is the set of nodes and $\mathrm{A}$ and $\mathrm{E}$ define two types of links which connect these nodes.

- $\mathbf{V}=\left\{\mathrm{V}_{1}, \mathrm{~V}_{2}, \mathrm{~V}_{3}, \ldots, \mathrm{V}_{N_{s}}\right\}$, where $\mathrm{V}_{i}$ is the set of leaves of the splittree. 


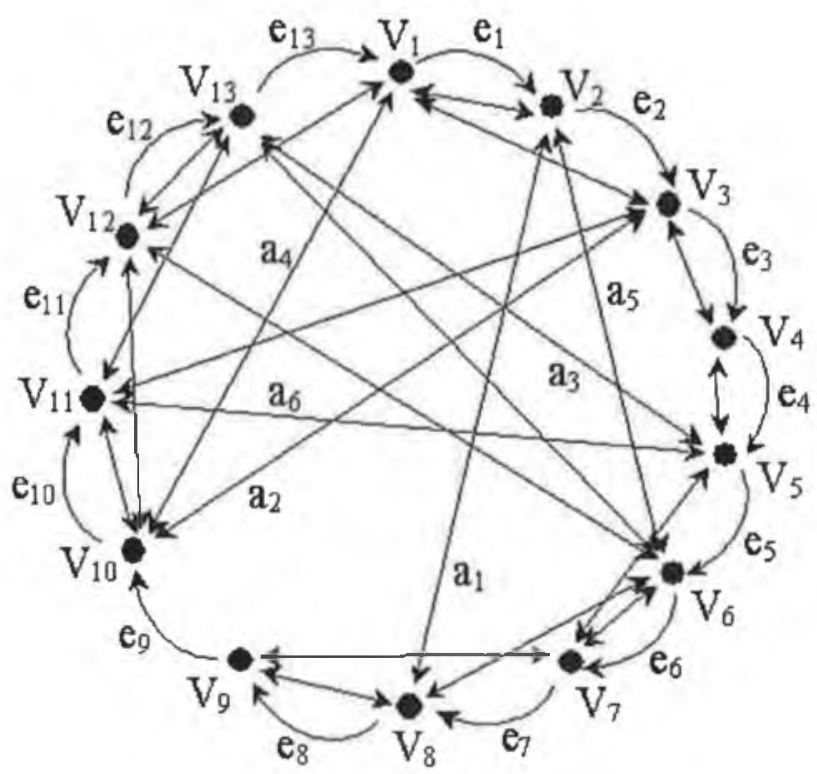

Figure 3.5: A typical example of mergegraph

- $\mathrm{A}=\left\{a_{1}, a_{2}, a_{3}, \ldots, a_{n}\right\}$, where $a_{i}$ is the set of adjacent links between $\mathrm{V}_{i}$ and $\mathrm{V}_{j}$.

- $\mathrm{E}=\left\{e_{1}, e_{2}, e_{3}, \ldots, e_{n}\right\}$, where $e_{i}$ is the unidirectional link between $\mathrm{V}_{i}$ and $\mathrm{V}_{(i+1) \operatorname{modn}}$. This forms the circular link list of nodes.

Figure 3.5 shows a typical mergegraph for the splittree and the image illustrated in Figure 3.3. The mergenode procedure traverse through the circular link list. The merger importance value for all the adjacent nodes were found and the region with smallest merger importance value was merged with the current node. All the newly merged nodes were delinked from the circular link list and the merging information and adjacency information for the current node was updated. This procedure was repeated until a stopping rule was satisfied. The pseudo code outlined in Figure 3.6 shows one such iteration. The method applied was a good representation of a split image and the graph derived from the splittree form the initial point for the merging procedure. During the merging procedure, the 


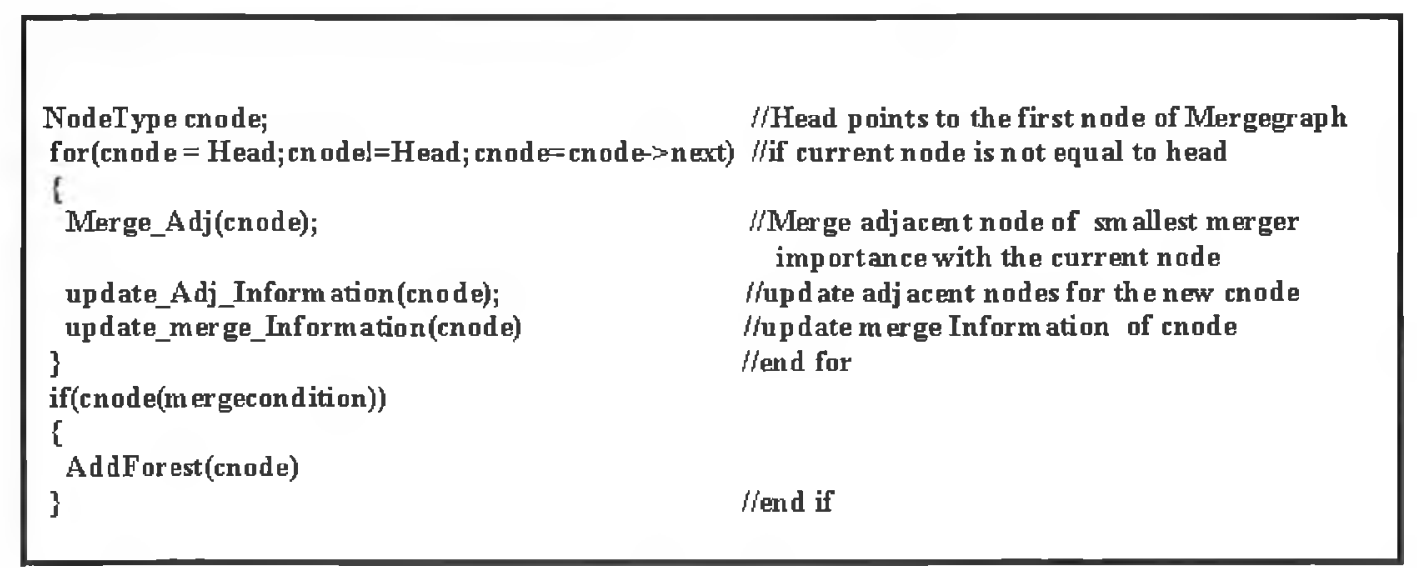

Figure 3.6: Pseudo code for the mergegraph approach

mergegraph breaks up into independent trees which is referred to as a forest data structure. Each tree in this forest represent a merged area.

Pixelwise Classification On completion of the splitting and merging operations, the image was processed for pixelwise classification. Pixelwise classification improves the localisation of the boundaries between the different textured regions. If an image pixel is on the boundary of at least two distinct textures i.e., the pixel is 4-connected to at least one pixel with a different label, a discrete disc with radius $r$ on the pixel was placed and the LBP/C or the DCT histogram over the disc was computed. The $\mathrm{G}$ distance between the histogram of the disc and the models of those regions, which were 4-connected to the pixel, were also computed. The pixel was relabelled if the label of the nearest model was different from the current label of the pixel and there is at least one 4-connected adjacent pixel with the potential new label. This condition improves smooth adoption of texture boundaries and decreases the probability of small holes occurring inside the regions.

If the pixel is relabelled, i.e., it was moved from an image segment to the adjacent segment, the corresponding texture models were updated. Only the pixels 
at which the disc was entirely inside the image were examined, hence the final segmentation result has a border of $r$ pixels wide. During the subsequent scan over the image, the neighbourhoods of the relabelled pixels were checked and classified. The process of pixelwise classification stops when no pixel is relabelled or maximum number of sweeps is reached. The value of $r$ is 10 and the maximum sweep depends on the segmented result after merging. A detailed explanation of pixelwise classification can be found in [21]. In all the experiments in this research work, pixelwise classification was tested with 4-connected and 8-connected pixels. The test results are discussed in chapter 5. In Method-II, LBP/C with colour features or DCT with colour features were used instead of $\mathrm{LBP} / \mathrm{C}$ or DCT histogram respectively. The pixelwise classification algorithm as described in [21] does not produce the expected results. Hence, a boundary refinement approach was devised as explained in the following section.

\subsection{Boundary Refinement}

A new boundary refinement algorithm was created and used for the improvement in the boundaries between various regions. A pixel is regarded as the boundary point if it is on the boundary of at least two distinct regions. i.e., its region label is different from at least one of its four neighbours. These points were identified and examined in the first sweep. For an examined point $P$, a discrete square with a dimension $d(\simeq 10)$ around the pixel was placed and the colour clustering histogram for this region was computed. Suppose the four neighbours of the point $P$ have $n$ different labels. The corresponding colour histograms for the different neighbouring points were calculated. The homogeneity of the square region and the $i$ th neighbouring region, $i=1,2, \ldots l \ldots n$ region was computed. The minimum of the similarity values is considered for relabelling i.e, point $P$ will be assigned to label $l$ if the homogeneity measure between the $l$ th region and the discrete square has the minimum value and the minimum value is less than 
the merging threshold condition. This condition is used to assess the minimum value and to achieve a more accurate segmentation. In the next sweep, the neighbourhood of those pixels which were relabelled in the previous sweep was considered. Reassigning pixels in this way improves the accuracy of the region. The process of boundary refinement continues until no pixels are relabelled. This boundary refinement algorithm considers the colour histograms and the merging threshold condition. This improved and enhanced the segmentation results.

\subsection{Summary}

A new colour texture image segmentation algorithm was implemented in this research work. The segmentation algorithm included feature extraction techniques for texture and colour followed by an unsupervised texture segmentation method. LBP and DCT were the two texture feature extraction techniques used. In addition to the statistical colour features such as mean, standard deviation, energy and entropy, an adaptive smoothing and unsupervised $k$-means clustering were the techniques used to extract colour features from the colour textured images. Two non-parametric tests were used to find the similarity between the regions. A boundary refinement algorithm was used to improve the segmented result. 


\section{Chapter 4}

\section{Methodology}

\subsection{Introduction}

This research work focusses on the development of a novel methodology for colour texture segmentation. The key contribution of this research work is the creation and the implementation of a novel methodology for colour texture segmentation. The first step in this approach is to identify a way to combine the colour and texture features, in other words the feature extraction for colour texture analysis. The two approaches followed in this study for feature extraction were,

- Texture features extracted from each colour plane separately. Method-I followed this approach.

- Texture features extracted from the intensity plane along with the extraction of pure colour information from the colour planes. This was carried out by using two methods, Method-II and Method-III, as described below.

Figure 4.1 explains the feature extraction approaches adopted and the colour texture segmentation methods followed, based on the feature extraction approaches.

This chapter describes the three methods developed in colour texture segmen- 


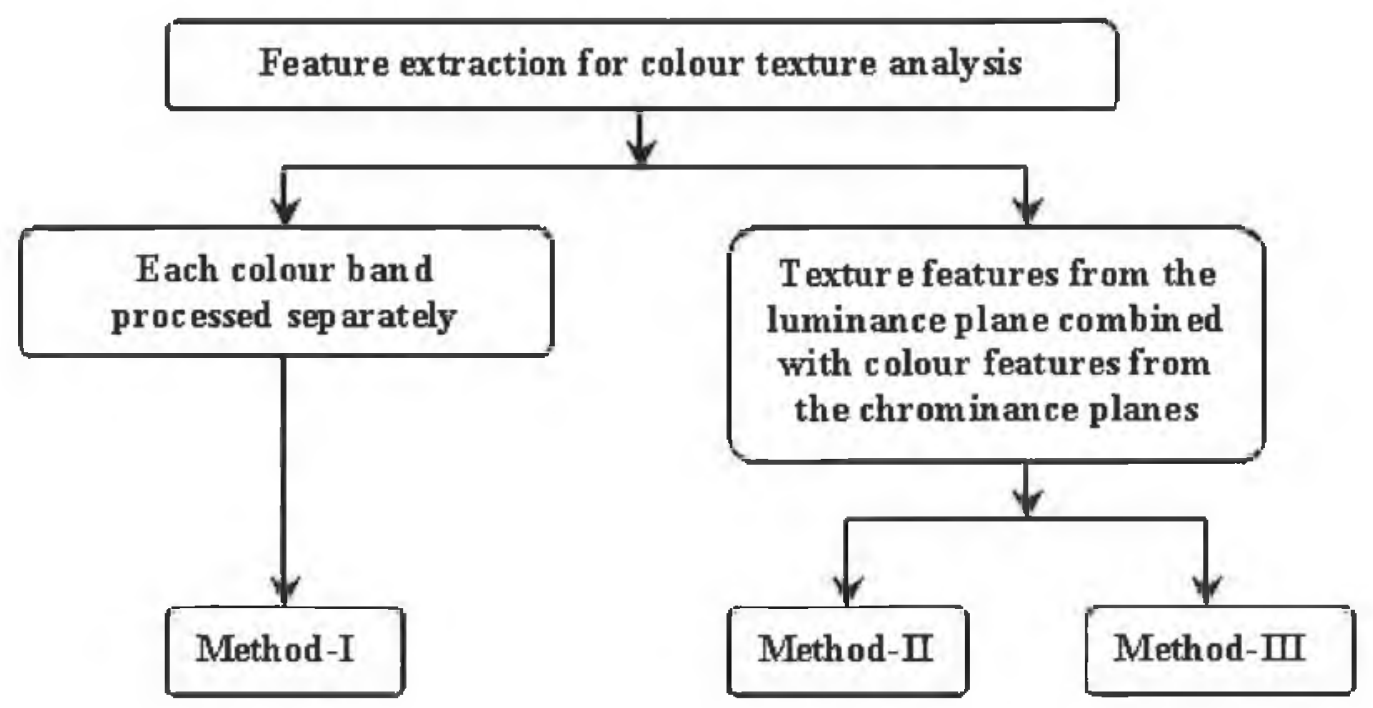

Figure 4.1: Flowchart representing feature extraction approaches and the colour texture segmentation methods

tation for integrating texture and colour information. Method-I used texture information from LBP/C or DCT techniques and the results were obtained in each colour plane individually. Method-II used texture information from LBP/C technique with statistical features from chrominance planes or DCT technique with statistical features from the chrominance planes or DCT technique with DCT features from chrominance planes. The colour texture integration used in Method-III is similar to Method-II that used the LBP/C or DCT features with colour clustering.

\subsection{Colour Texture Segmentation Methods and Description}

\subsubsection{Colour Texture Segmentation - Method-I}

This method is the extension of grey scale techniques to colour images. The segmented results were obtained in each colour plane separately. The colour 


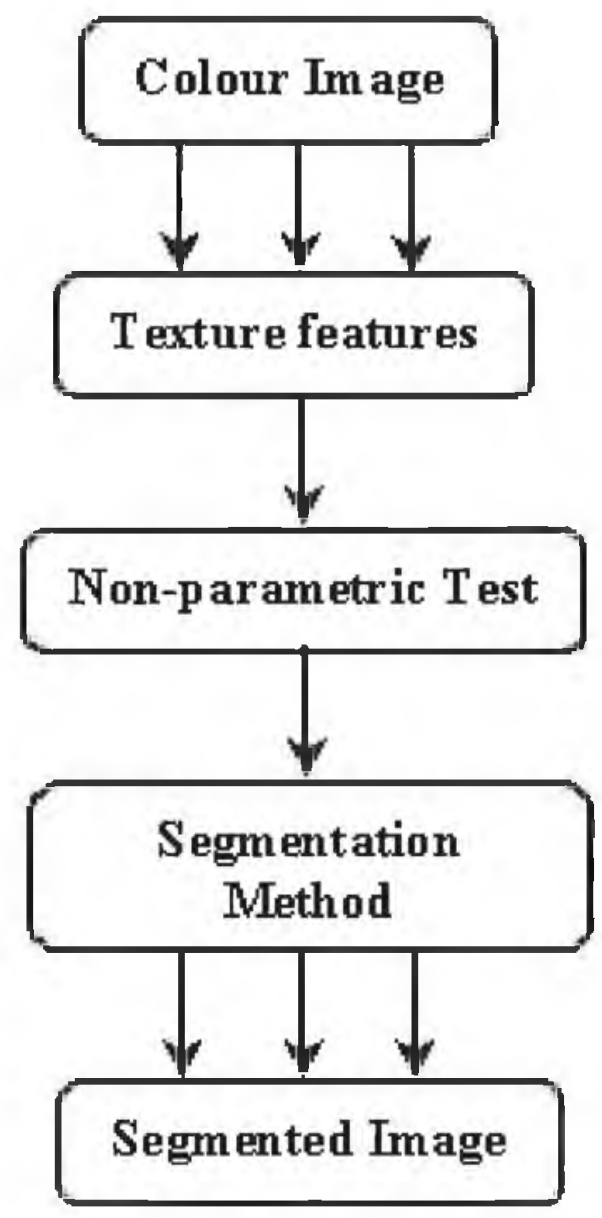

Figure 4.2: The colour texture segmentation for Method-I

texture segmentation procedure adopted in Method-I can be described as follows,

- Construction of the mosaic images using the VisTex colour texture image database.

- Conversion of the RGB images to HSI, YIQ, CIE-XYZ, CIE-LUV and CIE-LAB colour spaces.

- Application of the LBP/C to each plane, to extract the texture features or the application of the DCT based filter approach to grey scale images.

- The distribution of the texture features is used for texture discrimination. 
A log-likelihood pseudo-metric G-statistic is used to compare the feature distributions.

- An unsupervised texture segmentation procedure is employed to obtain the segmented results in each colour plane separately. The segmentation procedure consisted of three steps, the hierarchical splitting, the agglomerative merging and the pixelwise classification. Hierarchical splitting splits the image into blocks of roughly uniform textures based on a decision factor $\mathrm{G}$ value. Agglomerative merging is carried out to merge similar adjacent regions until a merging criteria is satisfied. This resulted in a blocky segmented image. In order to obtain an enhanced segmented result i.e., to improve the segmentation along the boundaries between the regions, a pixelwise classification algorithm is applied. This resulted in a final enhanced segmented image.

\subsubsection{Colour Texture Description for Method-I}

In Method-I, the standard texture analysis techniques, such as LBP/C and DCT, developed for grey scale images was extended to colour images. The limitation of using this method is the inadequacy of the use of colour information as the texture features are extracted from each colour plane separately. A comparative study on the LBP/C and DCT grey scale techniques was carried out in grey planes. The LBP/C technique was applied to different colour spaces such as RGB, HSI, YIQ, CIE-XYZ, CIE-LUV and CIE-LAB and the results were obtained in each plane individually. In LBP/C technique, textures were described using the 2D histograms of the LBP and the contrast which together formed 2048 bins. In DCT technique, textures were described using the 1D histograms of 2304 bins. The description of histograms discriminates the textures appropriately. The segmented results were obtained in each colour plane separately for $\mathrm{LBP} / \mathrm{C}$ and in grey plane for DCT using the unsupervised texture segmentation 
method.

Method-I used LBP/C to extract texture features from each of the colour bands separately. The extracted features captured the spatial structure of each texture and the features were computed from both luminance and chrominance planes. The segmentation was acquired in each plane independently. Method-I was adopted as a preliminary study for colour texture segmentation. The advantage of this method is its simplicity and straightforward feature extraction approach for colour texture segmentation. The segmented results from each plane gave an insight into the performance of luminance and the chrominance planes in various colour spaces. The disadvantage of this method is that the colour features cannot be explicitly used. Therefore, a method for combining texture and colour that can consider the strength and weakness of each feature type was necessitated. In order to make use of the colour features effectively together with the texture features, Method-II and Method-III were developed.

\subsubsection{Colour Texture Segmentation - Method-II}

Initially, the mosaic images were constructed using the VisTex colour texture image database. The images were converted from RGB to YIQ or HSI colour space using linear or nonlinear equations respectively. The conversion transforms the image into luminance and chrominance planes. The YIQ and HSI colour spaces are normalised before processing. The typical procedure is followed by two phases in Method-II, the feature extraction phase and the segmentation phase. A detailed explanation of the phases are as follows,

Feature Extraction Phase: The feature extraction phase has four steps,

- A $3 \times 3$ neighbourhood LBP approach or the $3 \times 3$ DCT based filter approach was applied to the luminance plane, to extract the texture features. The contrast from the LBP approach and the DCT values were quantised. 


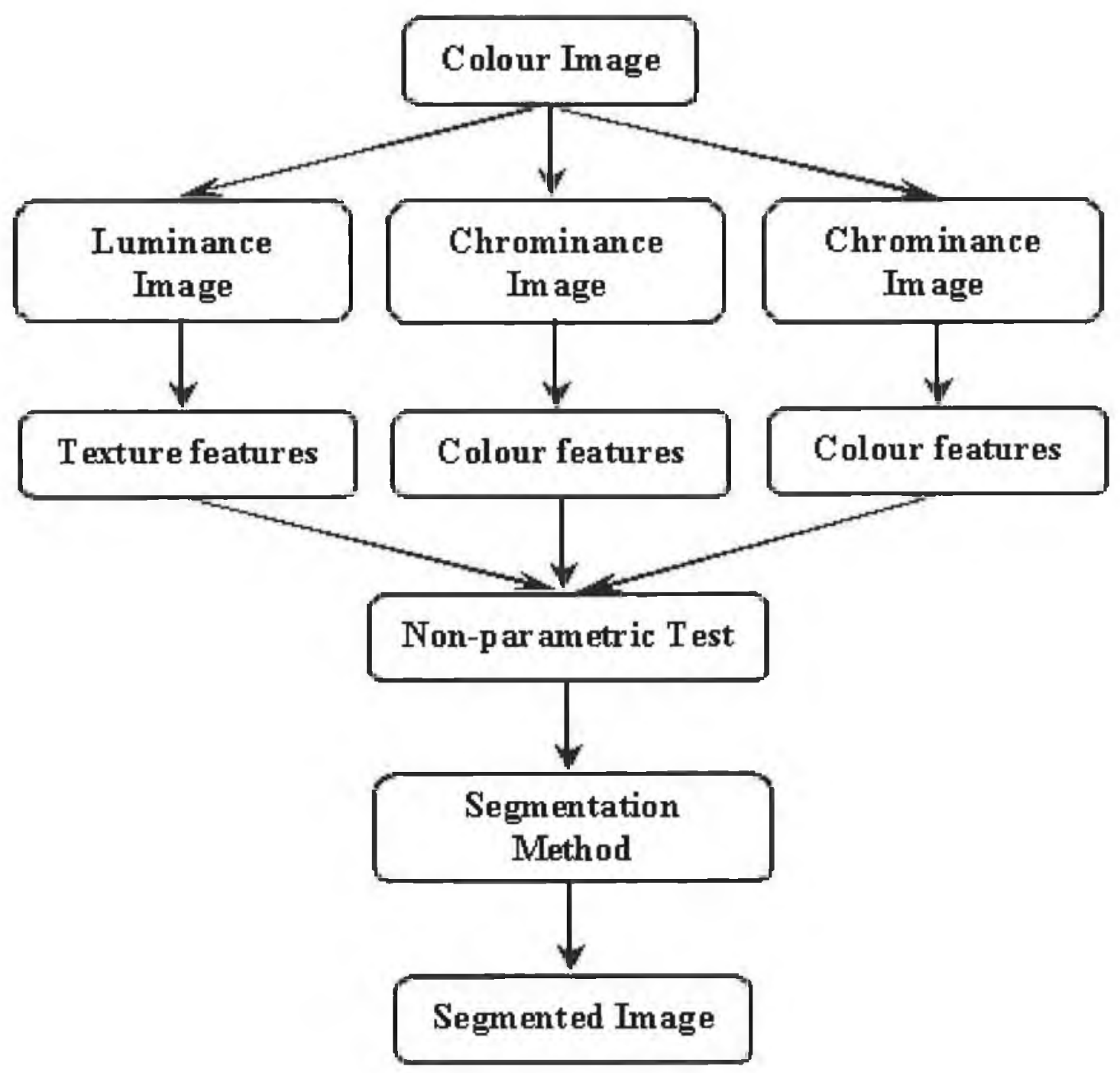

Figure 4.3: The colour texture segmentation for Method-II

- Statistical colour features such as mean, standard deviation, energy and entropy were derived from the chrominance planes. The statistical colour features were quantised in order to utilise the features effectively.

- Finally, the distribution of LBP/C or DCT and the distribution of the chrominance features were concatenated to obtain the distribution of colour texture features. 
- A non-parametric test G-statistic was used as a dissimilarity measure to describe the colour textures of an image region.

At the end of the first phase, the features from colour and texture were combined to obtain the distribution of the colour texture features. The distribution of the features were used in second phase of the Method-II to discriminate the colour textured regions.

Segmentation Phase: The segmentation phase consists of three steps, similar to Method-I, splitting, merging and the pixelwise classification, using colour texture features.

\subsubsection{Colour Texture Description for Method-II}

Method-II unified both colour and texture for colour texture segmentation. Much attention has been paid on the approach of fusing colour and texture. The implementation of LBP/C and DCT techniques were followed by the colour feature extraction and the addition of colour and texture features. The features derived encompasses both the structural patterns and the colour of the image. A detailed explanation of different combinations of colour and texture feature distributions explored in this study were as followed:

\section{$\mathrm{LBP} / \mathrm{C}+2$ Colour Features}

The texture regions are defined by two features, LBP and contrast. The LBP was combined with the contrast of the image and the $2 \mathrm{D}$ texture histogram was considered for texture description. This provides the pattern related information together with the contrast of the image. Each pixel in an image has an LBP and a contrast value. Hence each pixel can acquire any one of the $256 \times 8$ values. This $2 \mathrm{D}$ texture histogram is used in conjunction with the distribution of colour features for colour texture description. The colour features from the two colour 
planes were extracted and each feature was quantised to 8 bins. For example, the mean was extracted from I and Q planes in YIQ space and quantised to 8 bins in each plane. Then the colour features were added to the texture distribution. The colour and texture features forms a total of $2064\left(\left(\mathrm{LBP}^{*} \mathrm{C}\right)+\mathrm{C} 1+\mathrm{C} 2\right)$ bins, i.e., $(256 * 8+8+8)$ bins for this method, which provided a good discrimination of colour texture description. Analysing the distribution of the feature vectors within a region provides more information than the analysis on individual feature vectors. Hence, the colour texture description of a region is based on feature distributions. Similarly, standard deviation, energy and entropy were extracted from the colour planes, quantised and used for colour texture description. This method was tested in RGB, YIQ and HSI space using different statistical colour features.

\section{DCT +2 Colour Features}

The texture regions were described using nine mutually orthogonal 1D DCT masks, from the DCT based filtering approach. The quantised 9 DCT features were added and the sum of the 1D histograms from the 9 DCT filters were used for texture description. Similar to the previous approach with LBP and contrast, the colour features were extracted from the two planes and added to the texture distributions from DCT to form a total of $2320(9 \mathrm{DCT}+\mathrm{C} 1+\mathrm{C} 2)$ bins i.e., $(9 * 256+8+8)$ bins. The different colour features such as, mean, standard deviation, energy and entropy were compared and tested in different colour spaces.

\section{DCT +18 DCT Colour Features}

In the third approach 9 DCT features from both texture and colour planes were extracted. 9 DCT features were derived from the intensity plane in addition to 9 DCT features derived from each of the two colour planes adding to a total 
of 27 DCT colour texture features. This formed a 1D histogram of size 6912 $(9 \mathrm{DCT}+9 \mathrm{DCT}+9 \mathrm{DCT})$.

Three different combinations of feature distributions were proposed as colour texture descriptors in this study. The distributions of

- $\mathrm{LBP} / \mathrm{C}+$ statistical colour features

- DCT + statistical colour features

- DCT and DCT colour features

were used to measure the homogeneity of a pair of colour textured regions. The proposed method incorporates colour and texture features in a unified way, rather than simply extending the grey scale texture analysis techniques or analysing only spatial interaction of colours in a neighbourhood.

The unification of colour and texture in Method-II for segmentation is more effective. The advantage of this method is that the LBP/C or DCT technique is appropriately combined with simple statistical features. This computational framework for the colour texture segmentation procedure used additional colour information from the two channels together with the texture information. The segmentation is based on the discrimination of colour and texture features. The fusion of colour and texture features described the colour texture image effectively. In this method, the implementation of the pixelwise classification does not enhance the segmented result. This limitation is due to the inadequate discriminative information from the statistical features for the colour texture segmentation. Method-II does not remove noise and the segmentation along the border was not taken care. Hence, in order to increase the robustness of the approach to remove noise and to prevent over segmentation, Method-III was formulated. This method has a better description of the colour features. This method uses a normalised non-parametric test, adaptive smoothing and an unsupervised $k$-means 
colour clustering algorithm which results in a better segmentation.

\subsubsection{Colour Texture Segmentation - Method-III}

Method-III is a novel way of combining texture and colour information for the colour texture segmentation that makes the segmentation robust and efficient for different types of images. This method was performed in three phases. They were, hierarchical splitting, agglomerative merging and the boundary refinement. Similar to the previous method, initially the mosaic images were constructed and converted to YIQ or HSI space. The YIQ and HSI space were normalised before processing. The steps are as follows,

Hierarchical Splitting Phase: The hierarchical splitting phase tests the uniformity of the region. The goal of region splitting is to generate regions that form the input to a merging procedure that merges regions based on colour texture distributions. Initially, the features are extracted using feature extraction techniques, in which image information is reduced to a small set of descriptive features. This phase has the following steps,

- The LBP/C or DCT features are extracted from the average of the three planes in RGB colour space, Y plane in YIQ and from I plane in HSI colour space.

- The distribution of the texture features are used for texture discrimination.

- A Modified-Kolmogorov Smirnov (M-KS) non-parametric statistical test is used as a similarity measure to discriminate the texture distributions.

- A hierarchical splitting method is used to split the image based on the texture descriptions using the similarity measure.

This phase results in a split image using the texture features, that is used for merging in the next phase. 


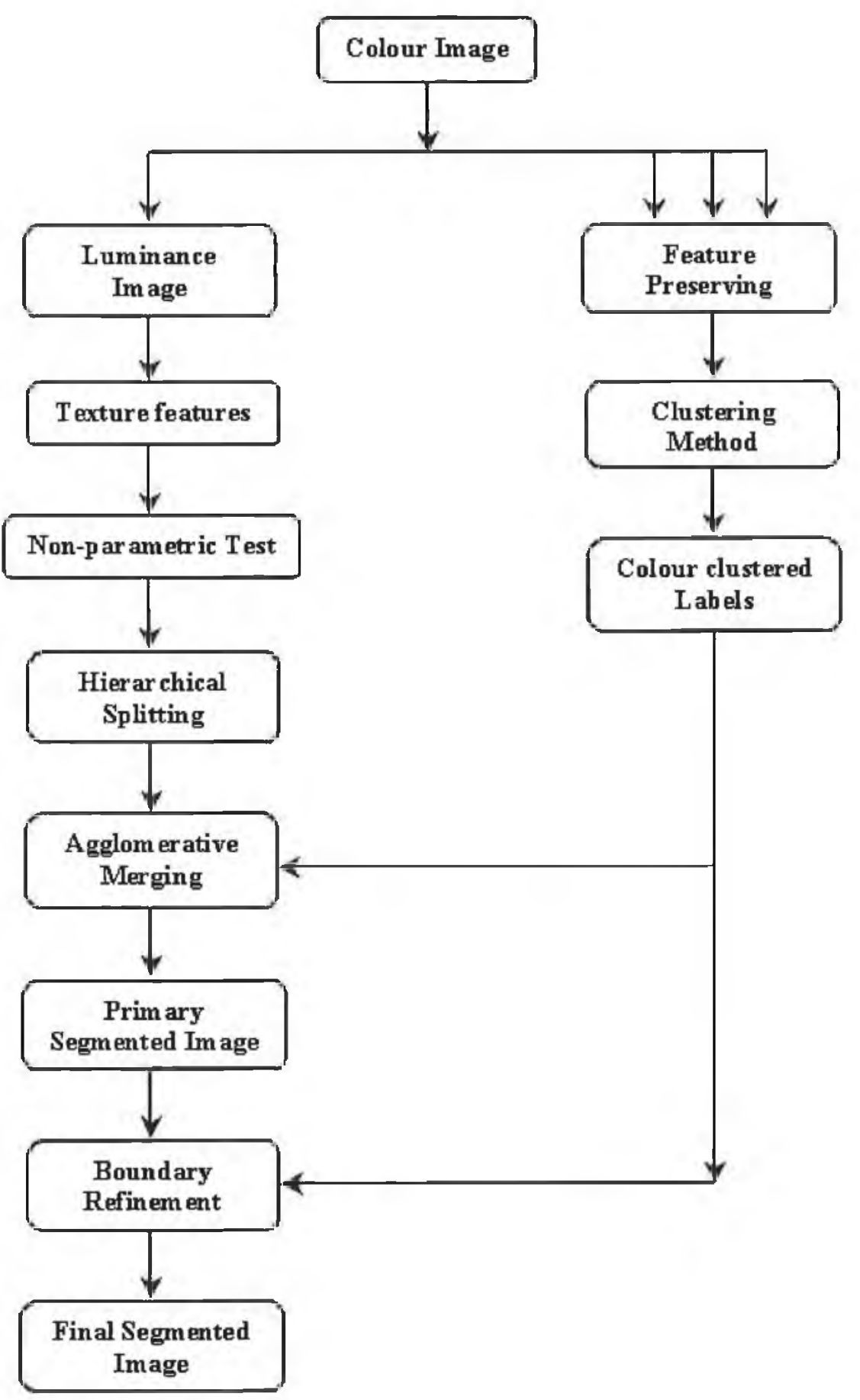

Figure 4.4: The colour texture segmentation for Method-III 
Agglomerative Merging Phase: In the second phase, unsupervised $k$-means clustering algorithm was used over the feature space to classify the patterns into their respective classes. The second phase has four steps,

- An adaptive smoothing is performed to preserve the features and to obtain a good segmentation along the boundaries. This technique removes noise and prevents over segmentation.

- An unsupervised $k$-means clustering algorithm is performed on the image to obtain the distribution of the colour clustered labels.

- Distribution of the texture features and the distribution of the colour clustered labels are used to describe the texture and the colour respectively. The distributions of colour and the textures was used to derive the merger importance value between two adjacent regions. The MI value was calculated using the M-KS statistic. Weights are included to both texture and colour features in the histogram and are computed using the histograms of the clustered labels.

- An agglomerative merging procedure based on the merging criteria determines the similarity between two different regions using M-KS statistic, producing the segmented image.

Boundary Refinement Phase The final step is to refine the boundaries of the image. A boundary refinement algorithm enhances the segmented result to obtain the final segmented image.

\subsubsection{Colour Texture Description for Method-III}

The developed framework described the colour texture in terms of the distributions of colour clustering labels and the distributions of texture features. MethodIII is an efficient approach to combine colour and texture for colour texture de- 
scription. The method of extracting texture features and the colour features were the same as in Method-II, but Method-III used a different non-parametric test, pre-processing and unsupervised $k$-means colour clustering to obtain the colour clustering labels.

\section{LBP/C with Colour Clustering}

Two features were proposed in this study to distinguish between colour textures, namely a colour clustering label histogram and an LBP histogram. The former approach is related to the distribution of colour clustering labels in an image region, while the latter is related to the distribution of LBP values in a texture region. Both of these distributions were derived from the image. LBP/C distribution is used for splitting. The $2 \mathrm{D}$ histograms of 2048 bins were used to split the image in the splitting phase. An unsupervised $k$-means clustering algorithm provided colour clustering labels (CL). In the merging phase, the MI value between the two regions is calculated as followed,

$$
M I=w_{1} \times M K S_{1}+w_{2} \times M K S_{2}
$$

where $w_{1}$ and $w_{2}$ represents corresponding weights for the LBP histogram and the colour clustered histogram respectively. $M K S_{1}$ and $M K S_{2}$ represents the M-KS statistic for the texture and the colour histograms respectively. The weights are calculated using the uniformity factor as follows,

$$
k_{j}=\max \left\{\frac{C L_{j}[i]}{N_{p}}\right\}, i=\text { number of clustered labels and } j=1,2
$$

where $k_{j}$ represents the uniformity factor for the sample and the model respectively. The numerator in equation 4.2 represents the colour clustered histogram for the region. The denominator $N_{p}$ represents the number of pixels in the corresponding regions. Appropriate weights were determined experimentally. If the difference between the $k_{1}$ and $k_{2}$ is less than 0.1 , i.e., both the sample and model weights are more or less the same, then the average of the k's is calculated i.e., 
$w_{2}=\left(k_{1}+k_{2}\right) / 2$ and the corresponding value is substituted to $w_{2}$. This indicates that the colour influences more than texture, hence colour statistic is given more weight. Then $w_{1}=1-w_{2}$. On the other hand, if the difference between $k$ 's is high both the texture and the colour are given equal weights and is taken as 0.5 .

\section{DCT with Colour Clustering}

9 DCT features were computed from the intensity plane and the features from each plane were quantised to 256 bins. The quantised DCT features were summed to form 1D histogram of size 2048. Similar to the previous approach using LBP/C and colour clustering, clustered labels were found using unsupervised $k$-means colour clustering. A similar procedure was followed to determine the MI values.

The fusion of colour and texture cues make the method more successful and enhances the reliability of the segmentation. The colour clustering and the pattern information significantly improved the segmentation accuracy. Unlike previous methods which used G-statistic, Method-III used M-KS statistical test. This non-parametric test is more appropriate in describing the statistics within the regions. Since M-KS is normalised, it is more advantageous than the G-Statistics. The M-KS statistic considers the sum of the absolute value of the discrepancies rather than the maximum of the absolute value of the discrepancies as defined in KS statistic. Hence, M-KS has larger discriminative power over the colour and texture features. The method integrates colour by clustering technique and texture by LBP/C or DCT technique. The clustering technique applies a specified objective criterion consistently to form the groups. The clustering technique reduces the number of features which in turn reduces the size of the feature distribution. This decreases the computational complexity of the algorithm.

A nonlinear, adaptive smoothing is used for noise removal and feature preservation. The adaptive smoothing considers the boundary factors and preserves the 
features which resulted in an efficient segmentation of the boundaries between regions. A novel boundary refinement algorithm was adopted to improve the border between regions. Method-III performed additional processes to derive useful features from the available information. This framework is capable of incorporating different kinds of information such as, colour clustering, smoothing, structural patterns which considers the issue of computational complexity and the segmentation in the boundaries. Also, the colour texture fusion provides an efficient segmentation. Hence this novel computational framework for colour texture segmentation is effective, reliable, consistent and robust.

\subsection{Summary}

The single band method or the Method-I utilises the spatial structure of the texture. The colour cue was not used in this method. The addition of colour features with texture features in Method-II and Method-III provided additional information about the details of the colour components. Method-II used simple statistical features to describe colour components and their spatial organisation. Method-III employed adaptive smoothing and colour clustering in conjunction with texture features. Among the three methods, Method-III used the distribution of colour labels with textures and provided a better segmentation. 


\section{Chapter 5}

\section{Results and Discussion}

\subsection{Introduction}

Vision Texture (VisTex) [27] is a collection of texture images used the by computer vision community. The experimental evaluation of the proposed methods was based on VisTex texture image database from MIT Media Lab. This consists of 19 different natural objects: brick, bark, buildings, clouds, fabric, flowers, food, grass, leaves, metal, paintings, sand, stone, terrain, tile, water, whereswaldo, wood and miscellaneous. The images from VisTex database were taken from many sources including examples from real world photography. The database has two main components:

- Reference textures - More than 100 homogeneous textures in frontal and oblique perspectives.

- Texture scenes - Images with multiple textures from real world scenes.

All images in the VisTex database were stored as raw portable pixmap (ppm) files. Currently there are two standard sizes for images in VisTex: they are $512 \times 512$ and $128 \times 128$. All images in the reference texture section and the texture scenes section are available in $512 \times 512$ and $128 \times 128$ sizes. 
In this research work, the images were first converted into bitmap images of 24 bit colour depth and true colour RGB representation. The mosaic of colour texture images of size $256 \times 256$ were constructed using a random selection of 4 different textures of size $128 \times 128$ from the reference texture database. Predominantly, possible homogeneous textures were selected to construct the mosaic images. The non-uniform textures inside a block such as clouds and the sky, flowers with the stem and textures with variations due to the dark and light colours were also considered for this colour texture analysis. Images with two textures and four blocks and images with 5 textures i.e., 4 blocks and a circle in the middle were also constructed. Some real world scenes of size $128 \times 128$ were randomly selected from texture scenes for the test database. 2 mosaic images, used by Mirmehdi and Petrou [86] and 2 natural images used by Panjwani and Healey [24] were also considered for the experiments. The mosaic images constructed using the reference textures have a spatial resolution of $256 \times 256$. Figure D.4M38 and Figure D.4-M39 (see Appendix D) are $184 \times 178$ and $184 \times 184$ in size respectively. The natural images from VisTex database are of size $128 \times 128$. Figure D.5-N10 and Figure D.5-N11 (see Appendix D) are of size $256 \times 256$. A total of 50 images consisting of mosaics and real world scenes were considered for the experiments. Figure D.1 - D.5 shows the collection of database images used in the experiments.

\subsection{Segmentation Results Using Method-I}

Initially the intensity components of the database images were examined. The $\mathrm{LBP} / \mathrm{C}$ or the DCT technique is applied to grey scale images. Method-I extends the LBP technique to colour images and the results were obtained in each plane separately. This method evaluated the performance of the LBP/C in greyscale images and also in individual colour planes. Method-I was adopted as an introductory approach as it is a simple and basic way of treating colour and textures. 
This method helped to familiarise with the implementation, programming and the colour texture processing approaches. This method was useful to observe the performance in greyscale and in the individual colour planes and also to find the effect of addition of colour in the succeeding methods. To begin with, the database images were tested in greyscale and also in RGB, YIQ, HSI, CIE-XYZ, CIE-LUV and CIE-LAB colour spaces and the results were obtained from each plane separately. The experiments were conducted in all the planes mentioned above. This was done to identify the best segmentation plane from the available segmented results, and also to select the suitable colour space for the subsequent methods. The results presented were obtained from a sample of 15 mosaic im-

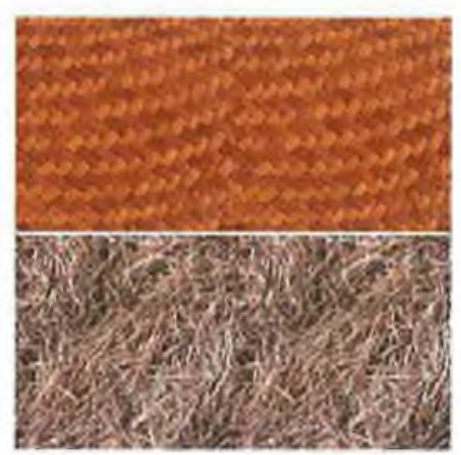

(MI-LBP-1)

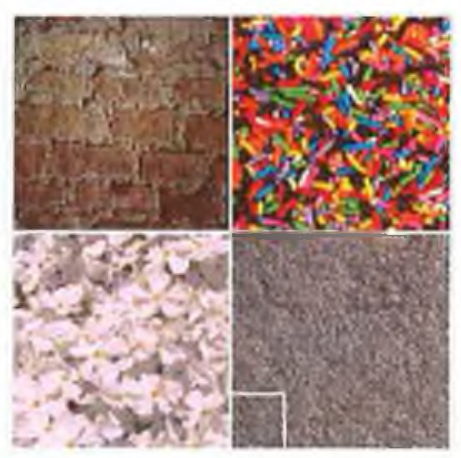

(MI-LBP-3)

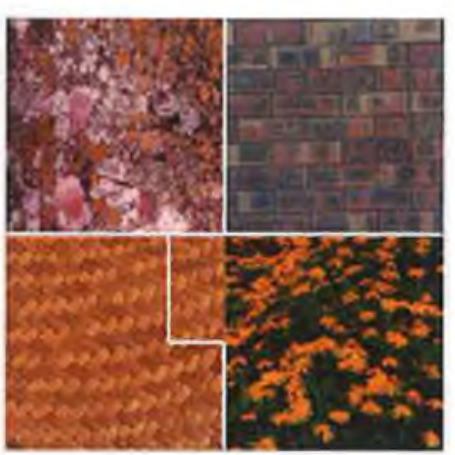

(MI-LBP-2)

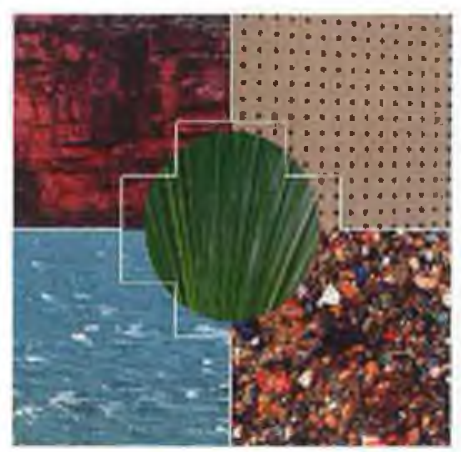

(MI-LBP-4)

Figure 5.1: Sample segmented results from greyscale images in Method-I using $\mathrm{LBP} / \mathrm{C}$ 
ages.

Some of the sample segmented results in greyscale images using LBP/C were shown in Figure 5.1. The segmentation results are superpositioned on the original images. Sample results showed in Appendix E were obtained from one of the RGB, YIQ, HSI, CIE-XYZ, CIE-LUV and CIE-LAB colour planes. Every plane resulted in different segmented results.

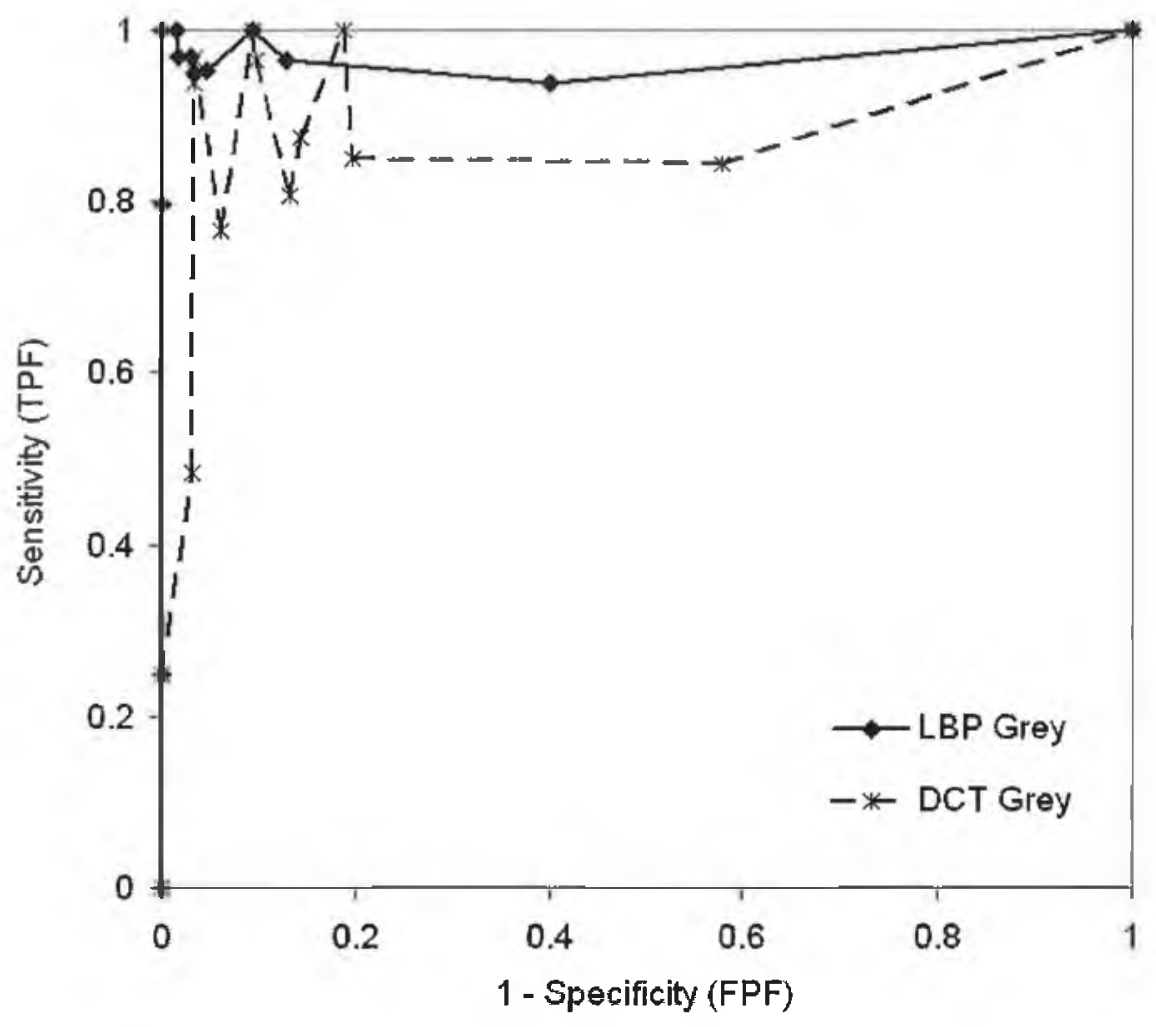

Figure 5.2: ROC curves representing the performance of grey scale LBP/C and DCT

In some images, for example in image MI-LBP-1 in Figure 5.1 the segmented result remained the same in almost all the planes. In Figure 5.1-MI-LBP-2 and Figure 5.1-MI-LBP-4 a large part of textures were merged incorrectly to different textures. In Figure 5.1-MI-LBP-3 the block was unmerged. This occurs due 
to insufficiency of intensity texture values to represent the textural variations and also due to the lack of colour information. The inclusion of the colour and combining colour and texture properly can enhance the segmentation result.

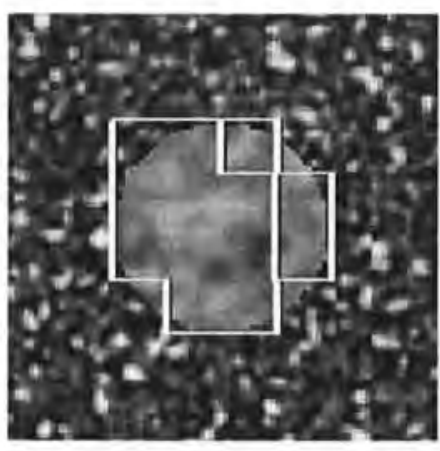

(a)

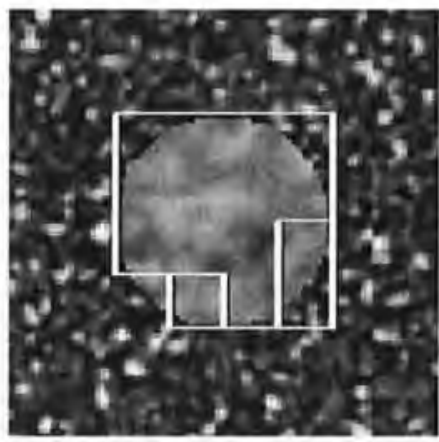

(c)

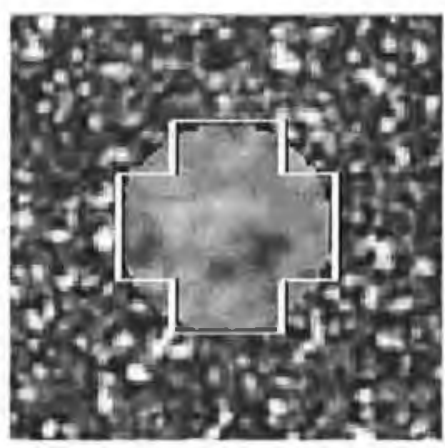

(b)

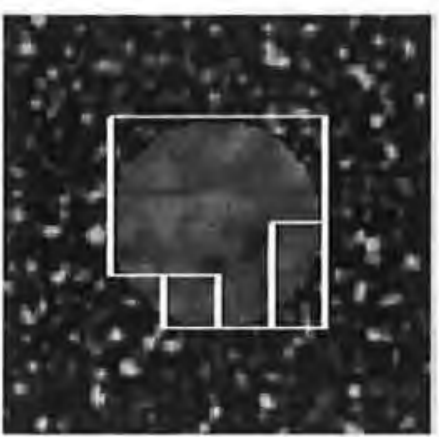

(d)

Figure 5.3: (a) - (d) Segmented results of a mosaic in grey scale and R, G and $\mathrm{B}$ planes respectively using $\mathrm{LBP} / \mathrm{C}$

As a quantitative analysis, the Receiver Operating Characteristic curve (ROC) is drawn to determine the efficiency of the method. The number of pixels correctly classified and the number of pixels misclassified in the segmented image were the discriminative features employed for drawing ROC curves. The ground truth of the test images was determined by manually drawing boundaries between different textured regions [68, 73]. The graph in Figure 5.2 demonstrates the tradeoff between true positive detection against the false positives. This shows the per- 


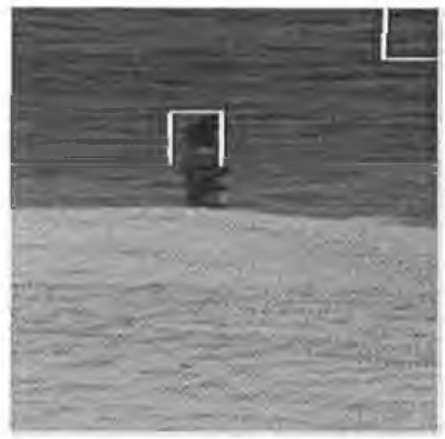

(a)

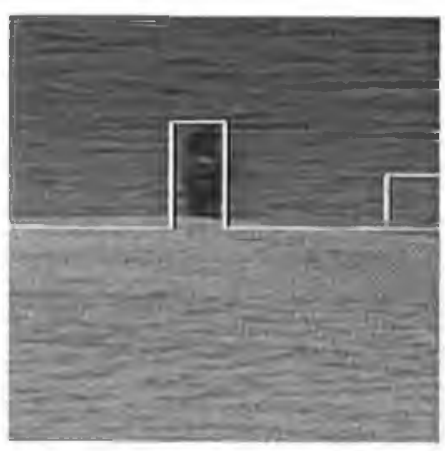

(c)

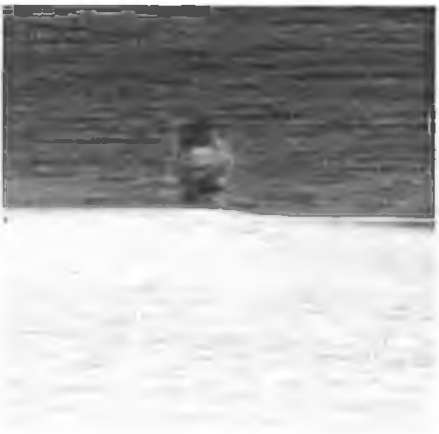

(b)

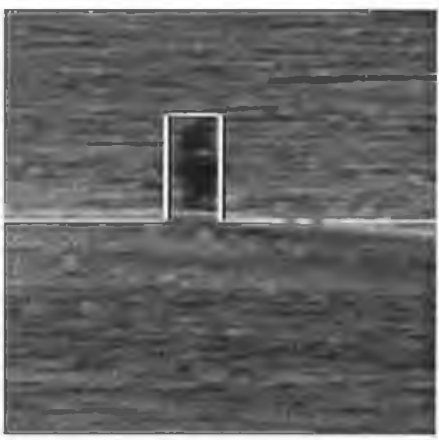

(d)

Figure 5.4: (a) - (d) Segmented results of bird in greyscale and R, G and B planes respectively using $\mathrm{LBP} / \mathrm{C}$

formance of the greyscale images. The graph illustrates that the LBP/C method for grey scale images performed better than the DCT filter based approach for grey scale images.

Figure 5.3 and Figure 5.4 illustrate the segmented results of a mosaic image and a bird image in grey scale and R, G and B planes. The segmentation results shows that the results were not the same for all the planes. The results in Figure 5.3 shows that the segmentation in $\mathrm{R}$ plane is much better than other planes, this is due to the presence of red components in the image. But Figure 5.4 illustrates that the segmentation is better in B plane. This is due to the fact that the beach area in the image having more blue components. The result implies that 
the segmentation depends on the colour components present in the respective planes.

On testing various planes in different colour spaces, the result in the planes cannot be judged, or it is not possible to draw a ground truth in a particular plane. Based on the investigation using various planes, it can be concluded that all colour planes performed equally well. From the study on various colour spaces it was observed that YIQ is the first to imitate the human visual system and HSI colour space is also based on the human visual perception. In addition, both the colour spaces have the advantage of separate processing of the luminance and the chrominance planes. In most of the cases where separate processing of achromatic and chromatic images are necessary, these colour spaces were used. Drimbarean et al. [14] performed an investigation on the performance of the colour spaces and suggested that none of the colour spaces proved to be superior but high classification accuracy was obtained using the YIQ colour space. This may be attributed to the fact that this transform is nearly orthogonal. Hence for the colour texture segmentation models discussed in Method-II and Method-III, YIQ and HSI colour spaces were employed for examination. Since RGB is the basic colour space the experiments were also conducted in this space in addition to YIQ and HSI colour space.

Figure E.2 (see Appendix E) represents the sample segmented results of natural images. Due to a number of small features in the image in Figure E.2, few small blocks remained unmerged. If the regions were unmerged or incorrectly merged then the error was generally overcome by using pixelwise classification method which enhanced the boundaries of the images. But the pixelwise classification adopted in this study could not correct the errors. This is due to the deficiency of colour information in this method. The inclusion of colour information is expected to make improvements in the next two methods. 


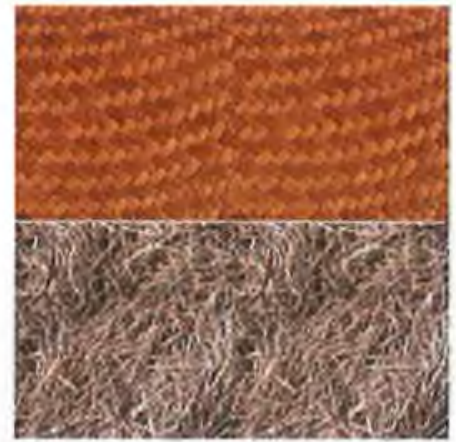

(MI-DCT-1)

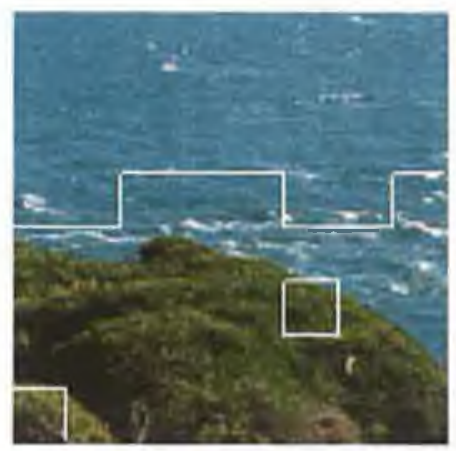

(MI-DCT-N1)

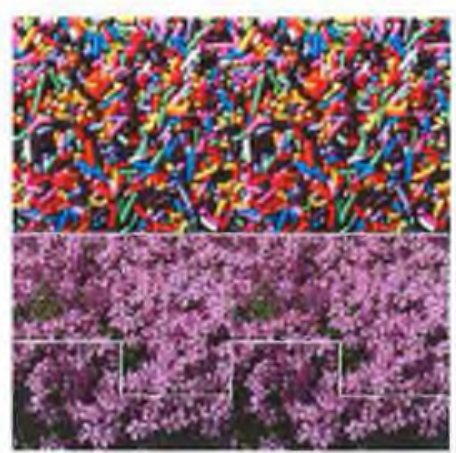

(MI-DCT-2)

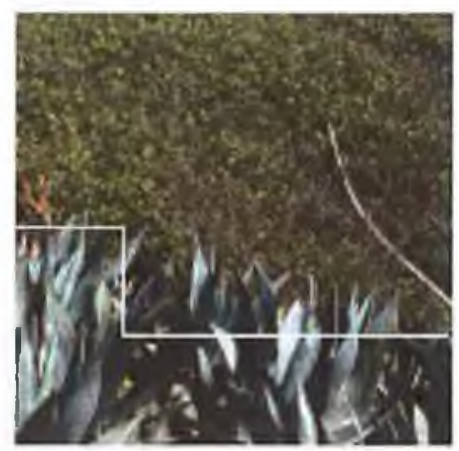

(MI-DCT-N2)

Figure 5.5: Sample segmented results of mosaic and natural images from MethodI using DCT

Figure 5.5 shows the segmented results of mosaic and natural images using DCT feature extraction technique in grey scale images. Figure 5.5-MI-DCT-2 shows the presence of some unmerged regions in the image. This was due to the variation in texture present in that region. Figure 5.5-MI-DCT-N1 and Figure 5.5MI-DCT-N2 shows that the boundaries were not accurate and are a few pixels away from the actual boundary. This was expected to be recovered by using the pixelwise classification.

Pixelwise classification was performed based on the neighbourhood of the boundaries, to obtain a refined segmented result. Generally the four connected neighbours of the boundary pixel was considered for classification. The segmented 


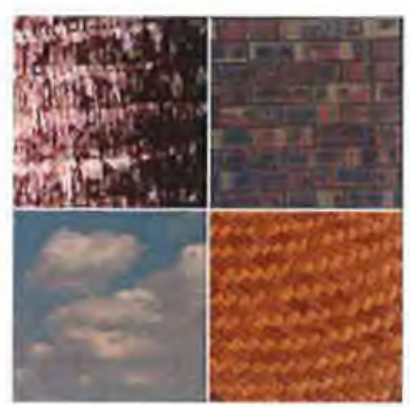

(a)

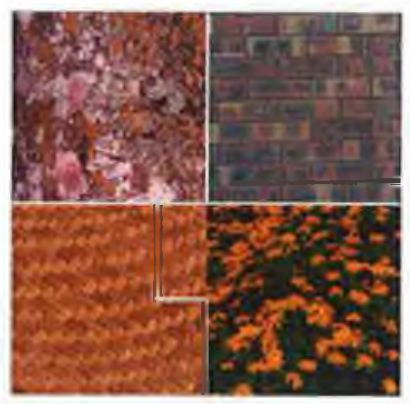

(c)

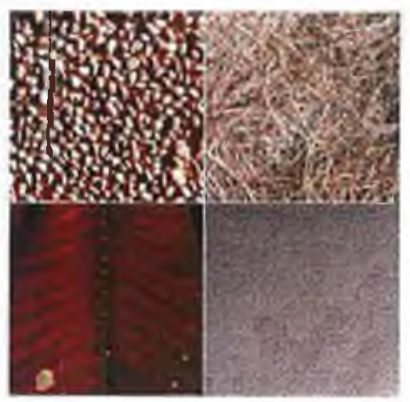

(e)

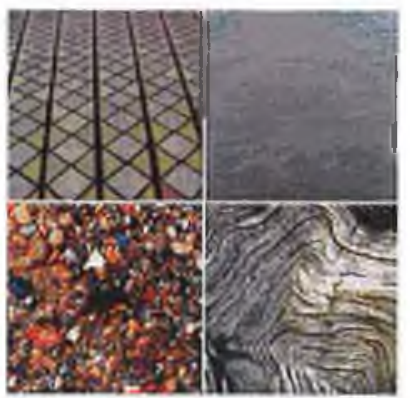

(g)

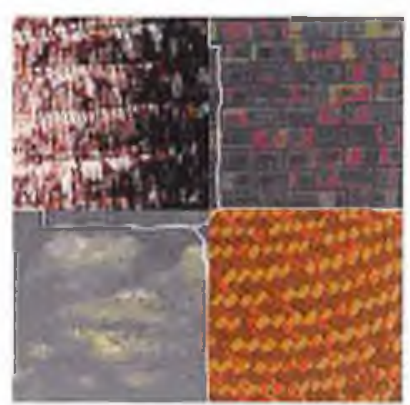

(b)

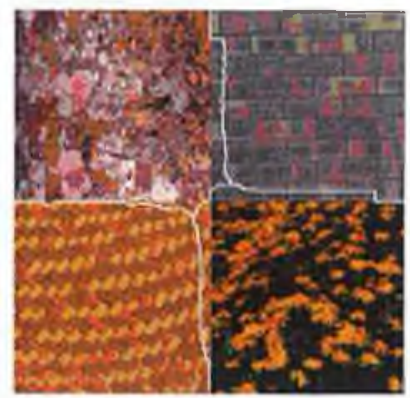

(d)

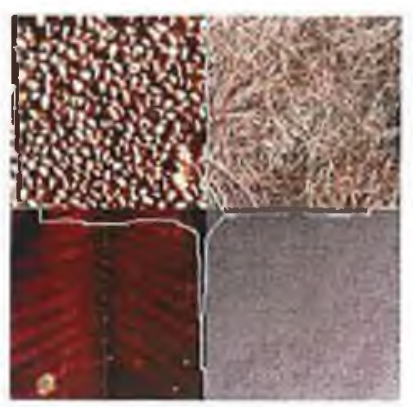

(f)

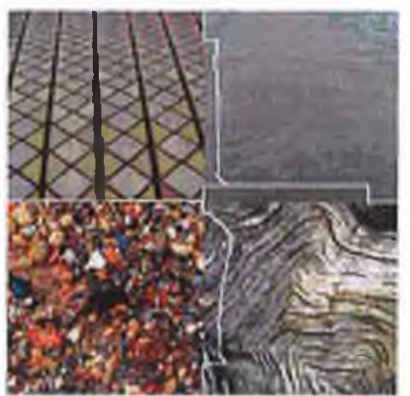

(h)

Figure 5.6: (a), (c), (e) and (g) shows the segmented results. (b), (d), (f) and (h) represents the corresponding pixelwise classified images 
results were tested by considering 2-connected, 4-connected and 8-connected neighbouring pixels. The cross neighbours of the 4-connected pixels were also tested. Not much difference in the results was found. Figure 5.6 (a)-(h), shows the segmented results and the pixelwise classified segmented results. The results obtained from the pixelwise classification method were not accurate and the border is a few pixels away from the actual boundary. Hence in order to get a better refinement of the boundaries, a new boundary refinement algorithm has to be adopted.

The evaluation of texture segmentation performance in different colour planes was not possible due to the presence of huge variation in the results and unavailability of the ground truth in each plane. Hence the segmentation performance of the Method-I in different planes was not evaluated.

\subsection{Segmentation Results Using Method-II}

This section analyses the segmentation results obtained using $\mathrm{LBP} / \mathrm{C}$ with chromaticity features and DCT with chromaticity features. The results presented were obtained from a sample of 15 mosaic images. Some of the sample segmented results using LBP and DCT with the chromaticity features are shown in Figure 5.7 and Figure 5.8 respectively. LBP/C with chromaticity features denotes LBP/C + colour features and DCT with chromaticity features denotes DCT-9 + colour features.

Figure 5.7-MII-LBP-1 and Figure 5.7-MII-LBP-2 represents a good segmentation. Figure 5.7-MII-LBP-3 and Figure 5.7-MII-LBP-4 shows blocky segmentation in the boundaries of the circle. This is expected to be overcome in the pixelwise classification stage. Some images such as Figure 5.8-MII-DCT-1 and MII-DCT-4 using DCT and chromaticity features shows results similar to that 


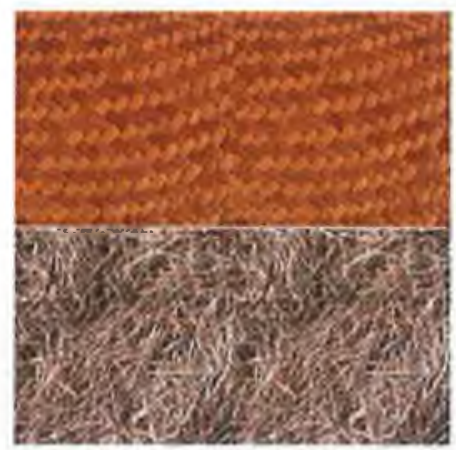

(MII-LBP-1)

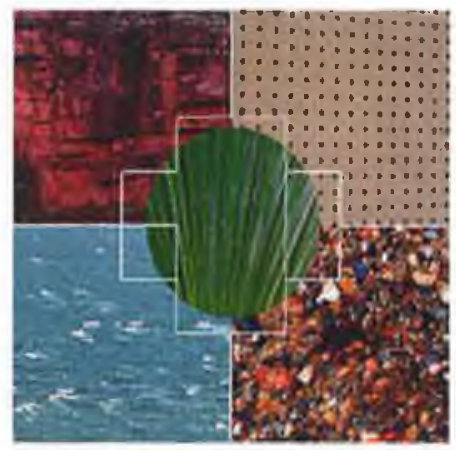

(MII-LBP-3)

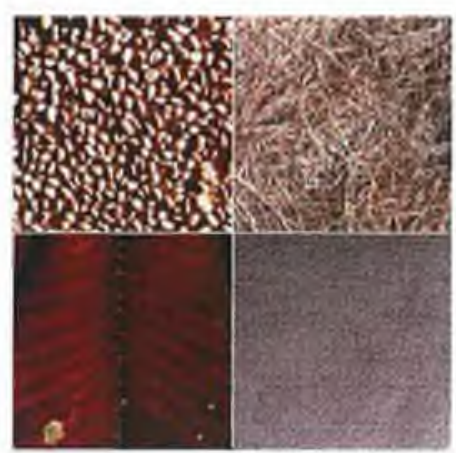

(MII-LBP-2)

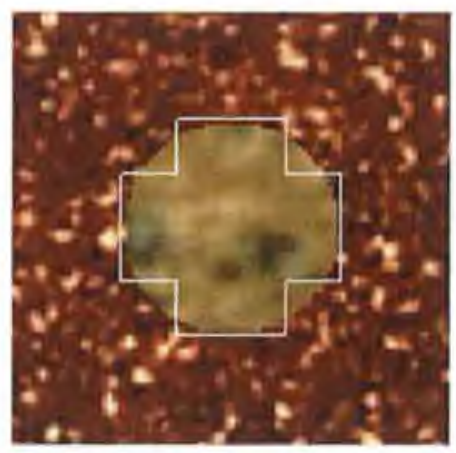

(MII-LBP-4)

Figure 5.7: Sample segmented results from Method-II using LBP/C and a chromaticity feature in RGB space

obtained using LBP/C and chromaticity features. Figure 5.8-MII-DCT-2 and Figure 5.8-MII-DCT-3 illustrates different segmentation results for the same image in $\mathrm{LBP} / \mathrm{C}$ and $\mathrm{DCT}$ approach respectively. The segmentation results using DCT feature obtained from texture plane with DCT feature obtained from colour plane (DCT-27 feature) were poor. Hence the results were not presented in this study.

For some images with square regions the results are accurate and $100 \%$ similar to the ground truth. The results shown here are obtained after agglomerative merging stage. Figure 5.8-MII-DCT-2 illustrates small unmerged blocks in the segmented image, except for a block which is unmerged the remaining blocks 


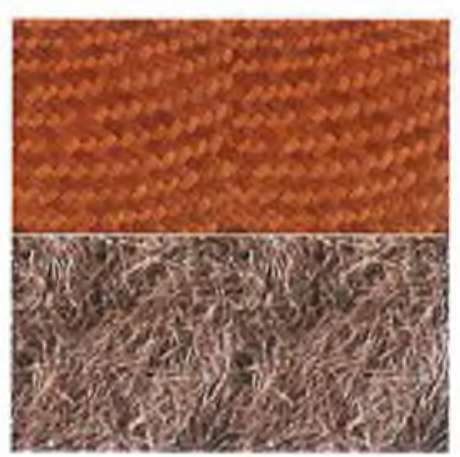

(MII-DCT-1)

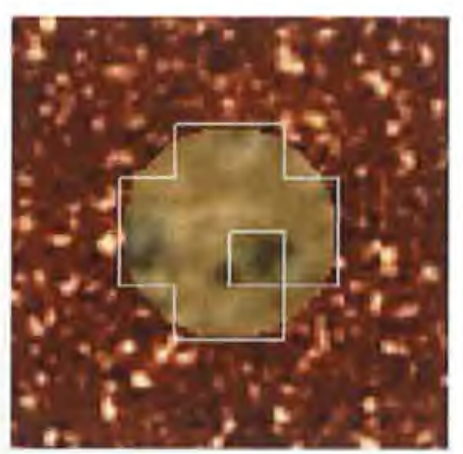

(MII-DCT-3)

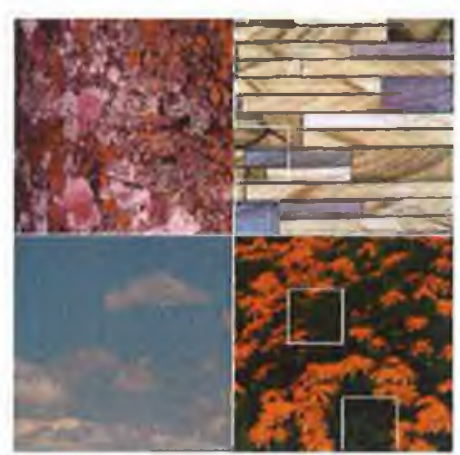

(MII-DCT-2)

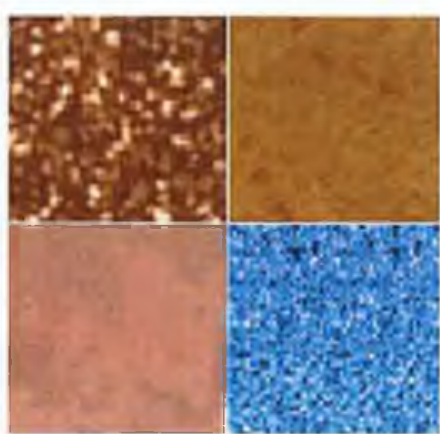

(MII-DCT-4)

Figure 5.8: Sample segmented results from Method-II using DCT and a chromaticity feature in RGB space

were properly segmented according to the variation in colour and textures. Most of the results are properly segmented, but some segmented images have false positive detections that are shown in Figure 5.8-MII-DCT-2 and Figure 5.8-MIIDCT-3. Sample segmented results of mosaics and natural images for LBP/C with chromaticity features and DCT with chromaticity features are presented in Appendix F.

\subsubsection{Performance Evaluation}

Various analyses were carried out to find the role of colour in colour texture segmentation. In order to evaluate the effectiveness of the proposed method, the 


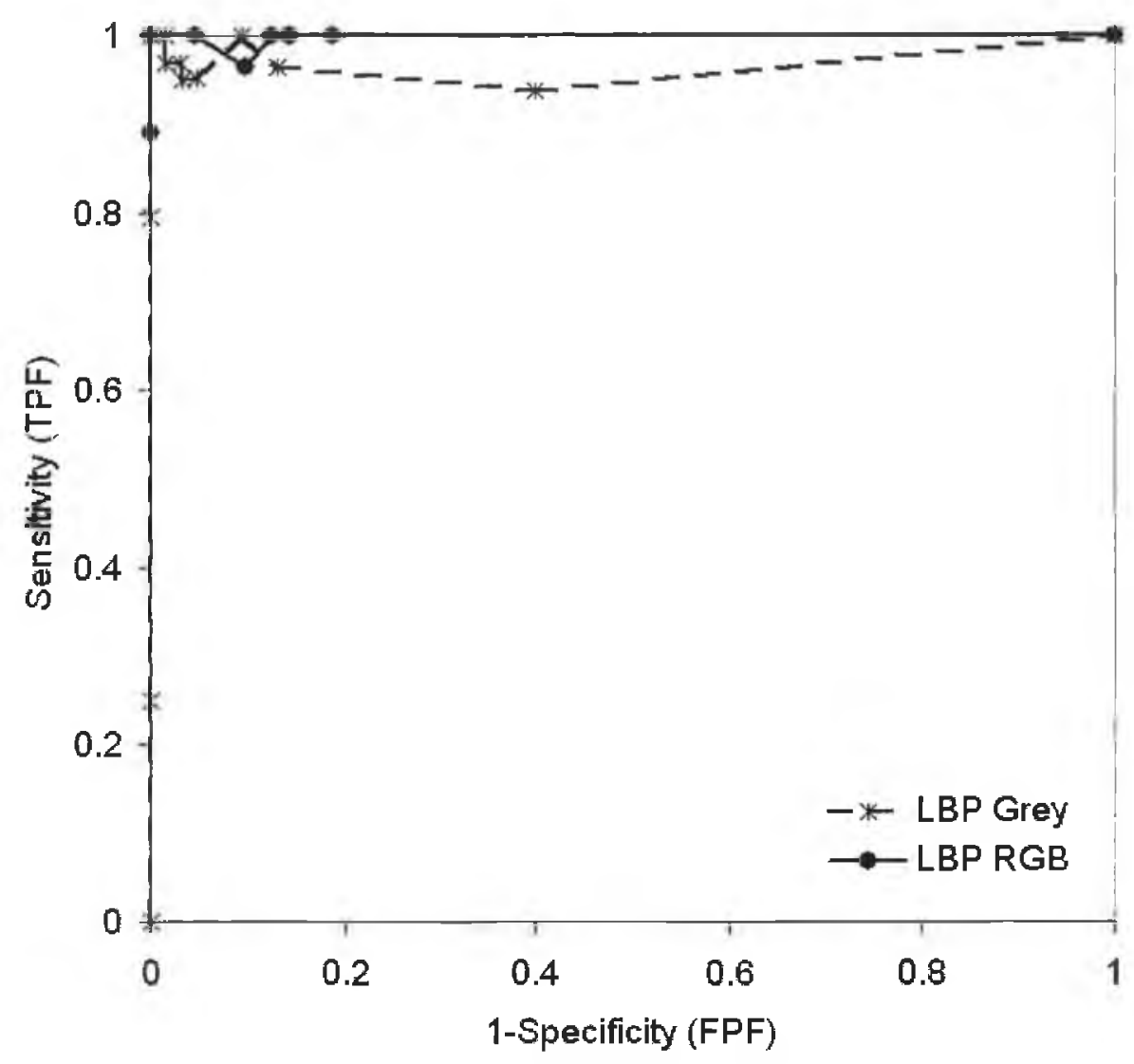

Figure 5.9: ROC curves for LBP/C approach in greyscale and LBP/C with statistical colour feature standard deviation in RGB space

database images were processed using the segmentation algorithm. The images were tested for a set of threshold values. The two parameters used for the splitting and merging were fixed at the values with minimum segmentation error. The values for split threshold and merge threshold were found to be 0.9 and 1.2 respectively. These parameters were fixed at the values with minimum segmentation error.

The first set of analysis estimates the performance of greyscale and colour images. As a preliminary test, segmentation results for greyscale LBP/C or DCT 


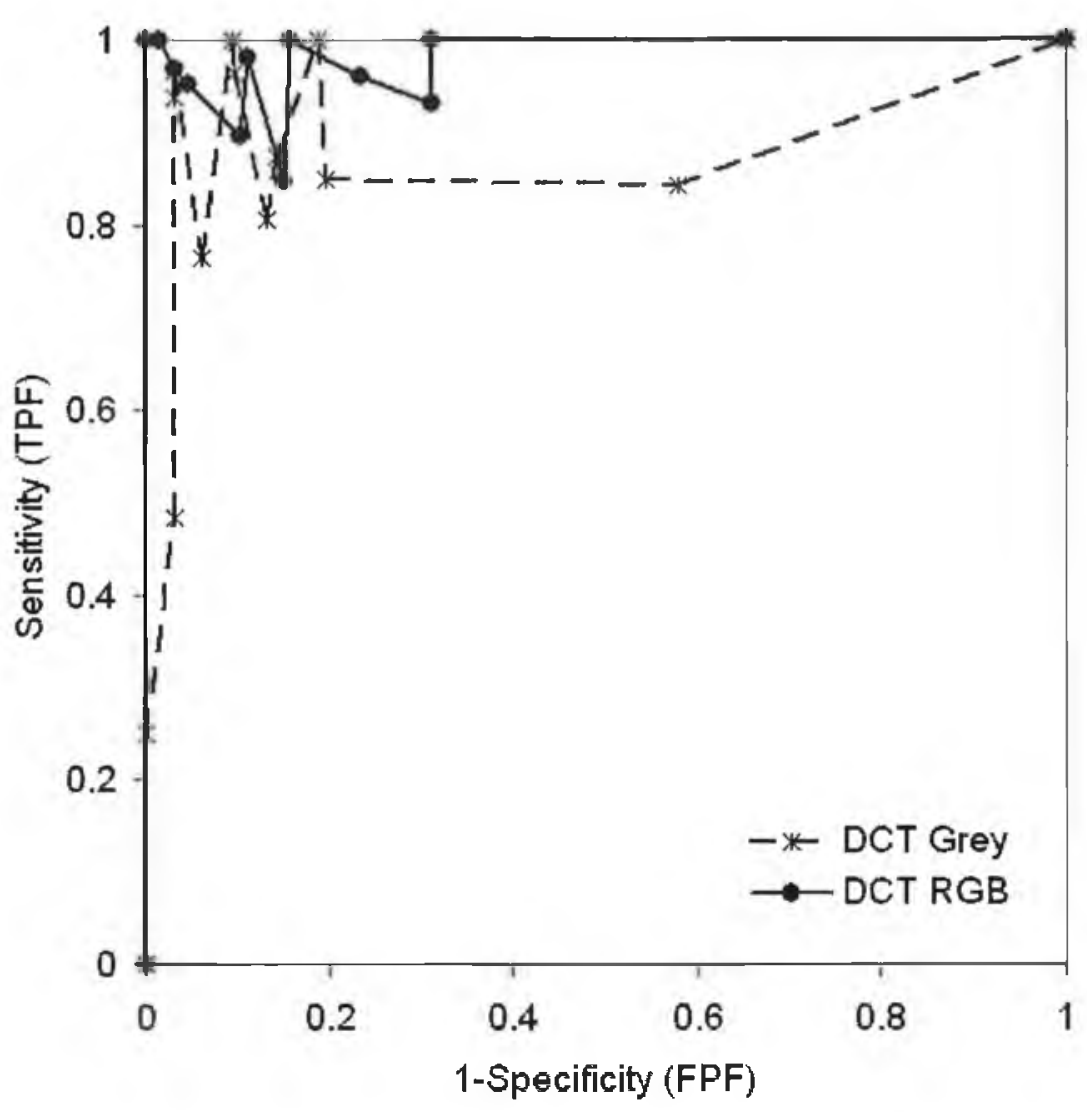

Figure 5.10: ROC curves for DCT approach in greyscale and DCT with statistical colour feature standard deviation in RGB space

and LBP/C or DCT with statistical colour feature standard deviation in RGB colour space were obtained. Based on the minimum segmentation errors the true positives against the false positives were plotted and the ROC curve was drawn. The graph in Figure 5.9 and Figure 5.10 illustrates that the segmentation performance increases due to the incorporation of colour information. The result shows an increase in the percentage of proper segmentation for the RGB space in both LBP and DCT approach. Though the graph in Figure 5.9 and Figure 5.10 shows a good sensitivity for some images it has nearly $10-20 \%$ of false positive fractions. 


\begin{tabular}{|l|l|l|l|l|l|}
\hline \multirow{2}{*}{ Technique } & \multirow{2}{*}{$\begin{array}{l}\text { Colour } \\
\text { Space }\end{array}$} & \multicolumn{4}{|c|}{ Chromaticity Features } \\
\cline { 3 - 6 } & & Mean & Std.dev & Entropy & Energy \\
\hline \hline \multirow{2}{*}{ LBP } & RGB & $95.3 \%$ & $87.5 \%$ & $83.5 \%$ & $84.7 \%$ \\
& HSI & $88.1 \%$ & $92.7 \%$ & $97.8 \%$ & $88.2 \%$ \\
& YIQ & $90.2 \%$ & $94.5 \%$ & $90.5 \%$ & $96.2 \%$ \\
\hline \multirow{2}{*}{ DCT } & RGB & $74.8 \%$ & $82.1 \%$ & $82 \%$ & $77.5 \%$ \\
& HSI & $75.8 \%$ & $76.9 \%$ & $82.8 \%$ & $77.1 \%$ \\
& YIQ & $83.5 \%$ & $74.1 \%$ & $84.6 \%$ & $73.8 \%$ \\
\hline
\end{tabular}

Table 5.1: Percentage of segmented results - Significance of colour features in colour texture analysis

The overall performance of the segmentation is shown in table 5.1. This is also shown as a graphical plot in Figure 5.11, Figure 5.12, Figure 5.13 and Figure 5.14. Using table 5.1, the second and third set of analysis were performed.

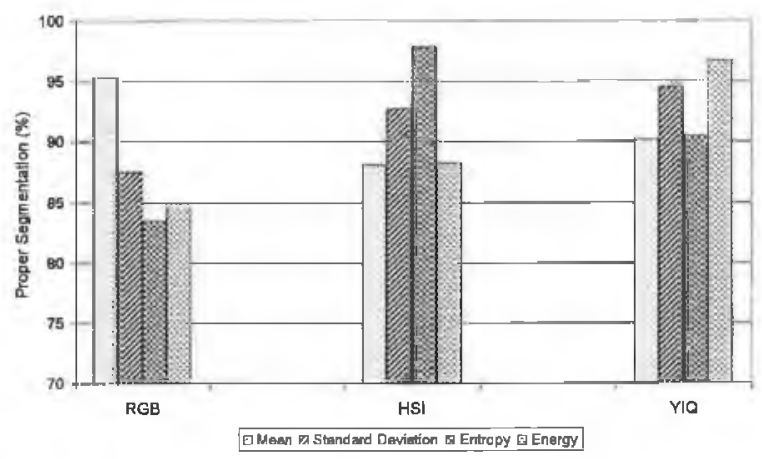

Figure 5.11: Bar chart representing the importance of chromaticity features using the LBP/C approach

The second set of analysis illustrates the significance of colour features in colour texture analysis. The different chromaticity features in various colour spaces were compared in this study. Standard colour features such as mean, standard 


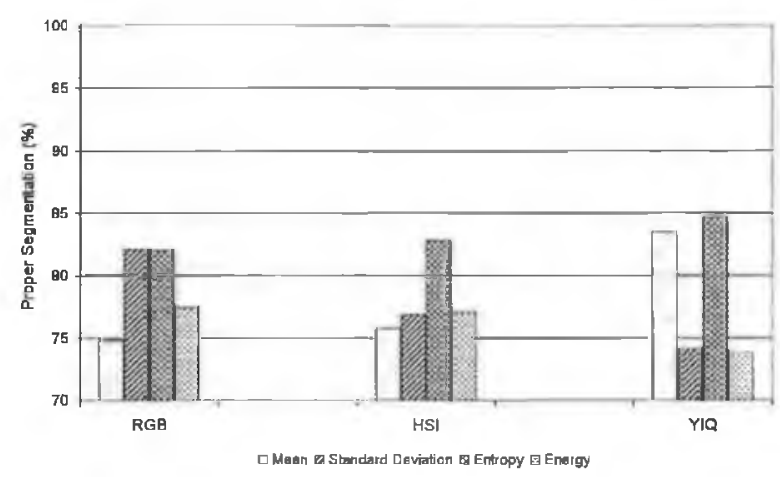

Figure 5.12: Bar chart representing the importance of chromaticity features using the DCT approach

deviation, energy and entropy were extracted from the colour planes together with the features from the intensity plane. The bar chart in Figure 5.11 and Figure 5.12 demonstrates the significance of each colour feature individually for colour texture segmentation. Comparatively, the results imply that the colour feature, entropy provides consistent results. However, none of the features proved to be always more effective than the others.

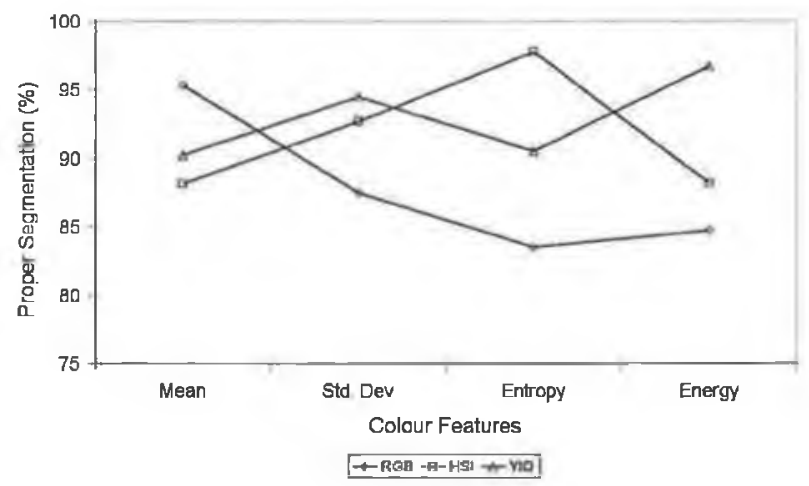

Figure 5.13: Comparison of the colour spaces in LBP/C

In the third set of analysis the comparison between the three colour spaces RGB, YIQ and HSI was carried out. From the results in Figure 5.13 and Figure 5.14 it is difficult to establish the best performing colour space. It can be seen that 
all the colour spaces have similar performances for LBP/C with colour features and DCT with colour features. This is in agreement with that obtained from the literature survey that none of the colour spaces proved to be superior.

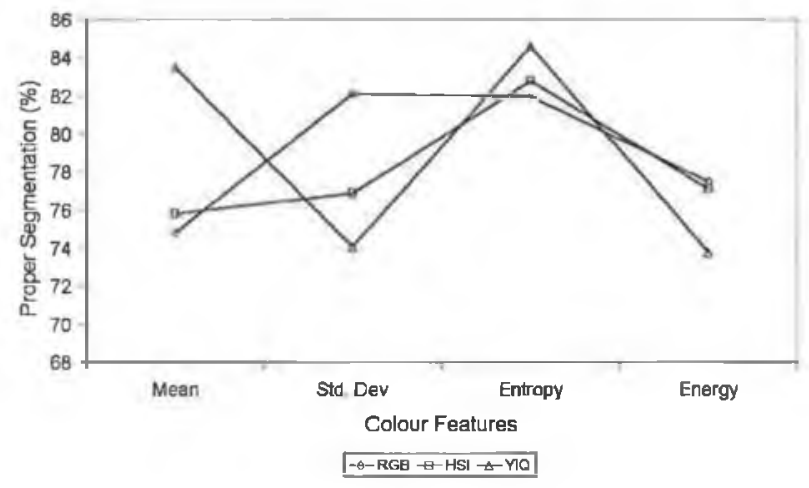

Figure 5.14: Comparison of the colour spaces in DCT

In the fourth set of analysis a comparative study for the three different approaches was undertaken (see Figure 5.15). The three different feature extraction approaches can be ranked according to their variability across different features. Among the three approaches LBP + colour features out performed the DCT-9 + colour features and DCT-27 feature approaches.

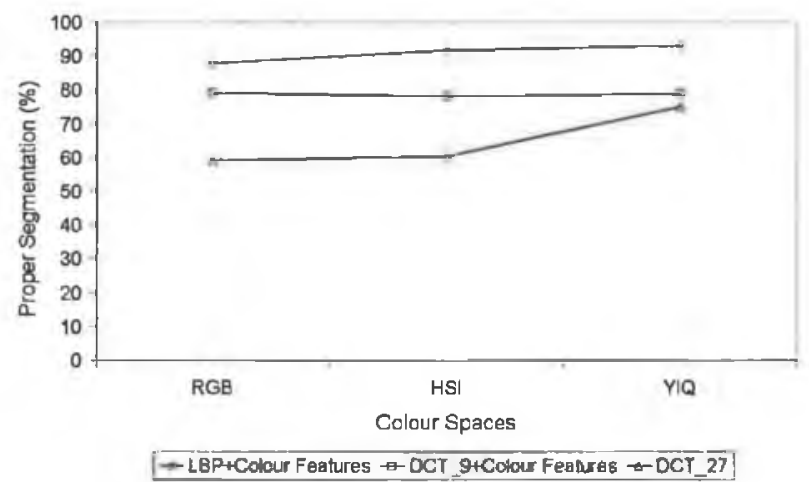

Figure 5.15: Comparison of the different approaches in Method-II

The best average percentage of the correct segmentation across the three different methods is obtained using $\mathrm{LBP} / \mathrm{C}+$ colour features with an average percentage 
of $90 \%$. The outcome for DCT-27 is a poor segmentation and hence the comparison was discarded for the colour space. The advantage of the LBP approach is its computational simplicity. The DCT approach uses a small bank of filters that results in a low feature dimensionality. The poor performance of DCT-27 may be due to the increase in dimensionality of the feature vectors that results in statistical instability of the histograms evaluated by the G-statistics.

\subsubsection{Influence of Texture and Colour}

The influence of colour in colour texture segmentation is shown in Figure 5.16. Figure 5.16 [a, c, e, g] represents the colour difference within the images using the adaptive smoothing and the $k$-means colour clustering and Figure $5.16[\mathrm{~b}$, $\mathrm{d}, \mathrm{f}, \mathrm{h}]$ shows the segmented results using Method-II. Sample segmentation results are presented in Figure 5.16. The result in Figure 5.16 (b) shows small unmerged blocks of texture. This occurs due to the difference in the colour components within a textured region. The image in Figure 5.16 (d) consists of bark, brick, cloud and fabric. Here the bark texture (top left quadrant of the image) is identified as two different regions. It is evident from the segmented image that the texture exhibits a significant colour difference. The application of the segmentation algorithm correctly identifies these regions, which is in agreement with the human visual system. This shows that colour plays a vital role in the experiments.

In Figure 5.16 (f) the tile and the water in the first and second quadrant were properly segmented based on the influence of colour in the image. In Figure 5.16 (h) a small portion of the leaves were segmented separately which gives a good evidence for the importance of colour and texture in the region. The brightness shown in the leaves is correctly identified and separated. The features derived encompasses both the structural pattern and the colour of the image. Colour 


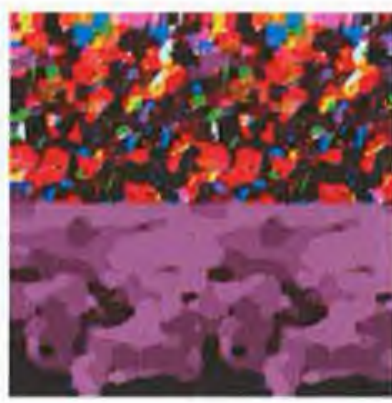

(a)

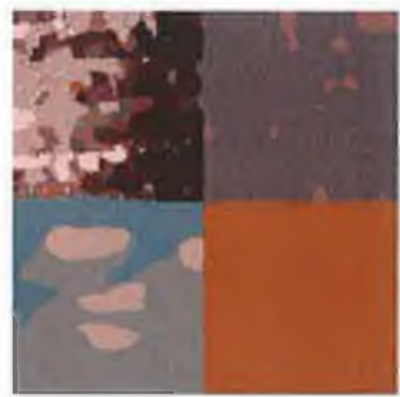

(c)

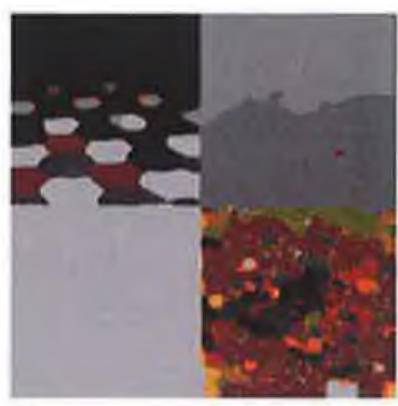

(e)

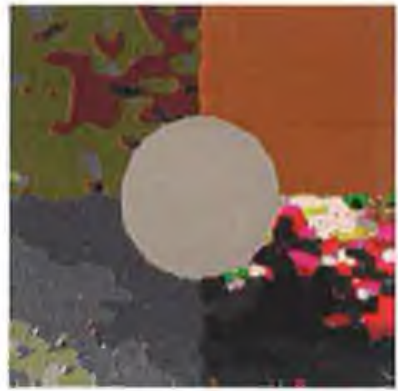

(g)

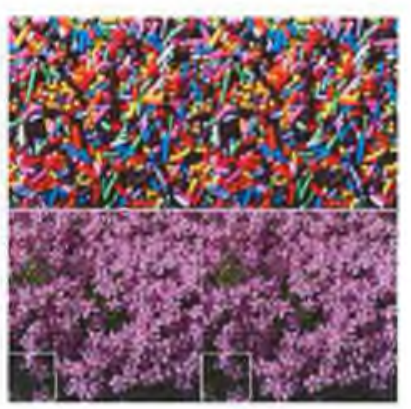

(b)

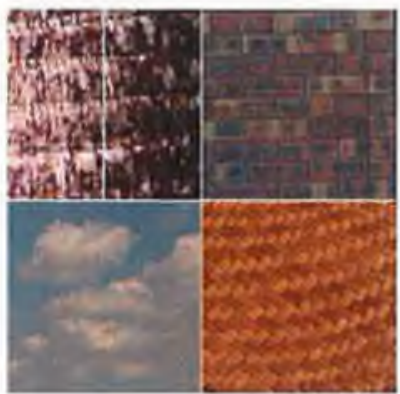

(d)

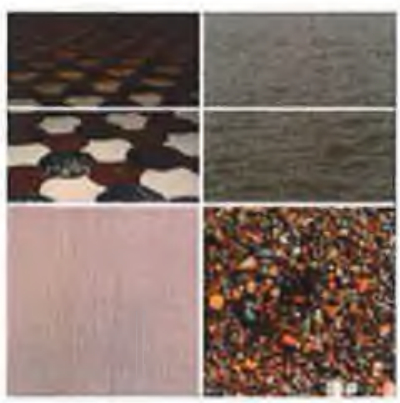

(f)

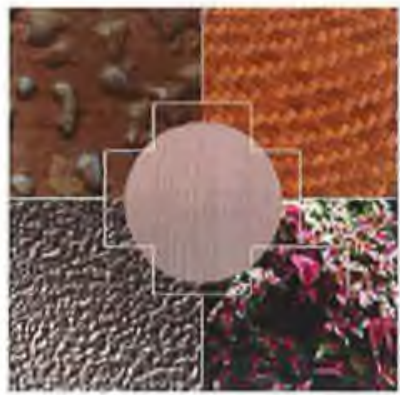

(h)

Figure 5.16: Influence of the colour and texture 
is a useful property, which adds information to images compared to greyscale images. Colour images have information about the brightness of each pixel in addition to the information such as hue and saturation.

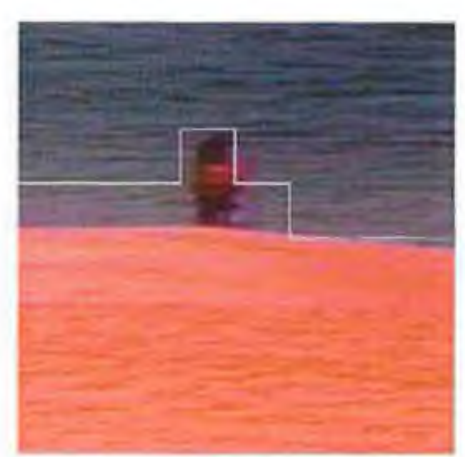

(MII-LBP-N1)

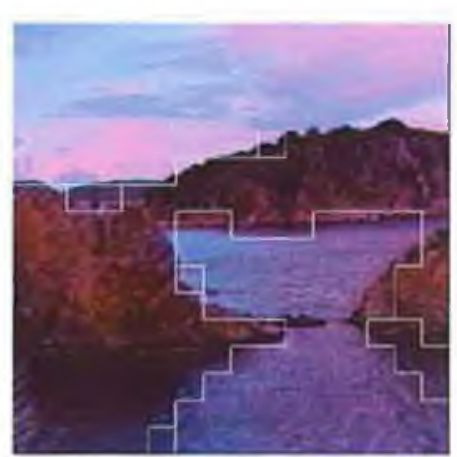

(MII-LBP-N2)

Figure 5.17: Sample segmented results of natural images

The segmentation results of natural images are presented in Figure 5.17. The beach image in Figure 5.17-MII-LBP-N1 is defined by regions with sand, water and a bird where darker and lighter regions can be noticed. The stopping rule used for these experiments allows the merging of the small components in the bird into a single region. Different stopping rule parameter and the blocksize until which the splitting is performed forms a vital part in the segmentation. Figure 5.17-MII-LBP-N2 shows the segmentation of a rock image. This image consists of regions of sea, rock and sky. In this example, it can be observed that the three regions were segmented distinctively. A portion of sea is merged with rock due to the similarity in colour components in that region. This clearly reveals the role of colour in colour texture segmentation.

From the above experiments it is apparent that colour plays a major role in texture segmentation. Effective inclusion of colour in colour texture analysis improves colour texture segmentation result.

The segmented images were tested for pixelwise classification. A pixelwise clas- 
sification similar to Method-I was considered except for the use of $\mathrm{LBP} / \mathrm{C}+$ colour features or DCT + colour features instead of simply using the features from the intensity plane. The results were similar to the results obtained from Method-I and does not perform to expectation. There are a large number of pixels that are not segmented properly and the segmentation boundaries are far from the true boundaries (up to 10 pixels). This is due to the fact that the colour texture segmentation performs very well within a region, but it has problems on the boundaries between regions. The poor performance of the pixelwise classification algorithm is evident from the results in Figure 5.18. A new boundary refinement technique was adopted in Method-III and the results are discussed in section 5.4 .

In Method-II, colour texture is described by LBP/C or DCT + colour features. This method concatenates the colour features to the textures in a simple manner. This method performs effective segmentation. However, the features are not sufficient to achieve proper segmentation in the boundaries, to obtain enhancement of the boundaries. To overcome these problems another method was followed which describes the region, based on the similarity measure using the distributions of texture and the distributions of colour. 


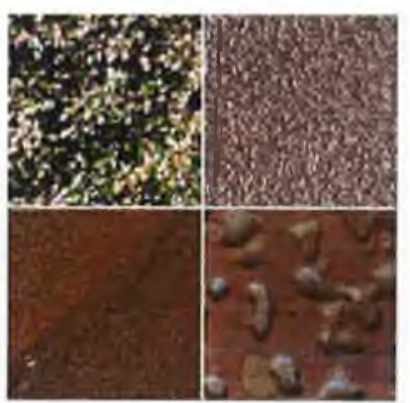

(a)

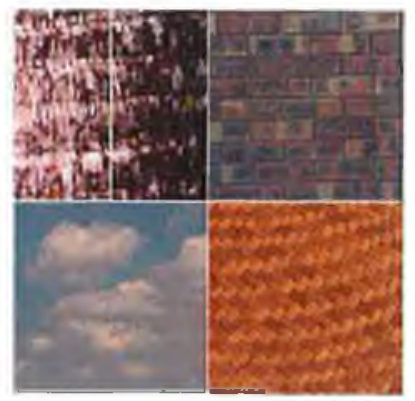

(c)

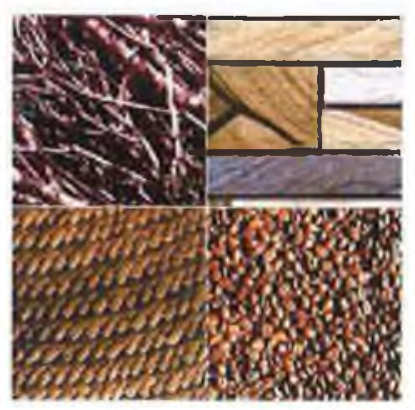

(e)

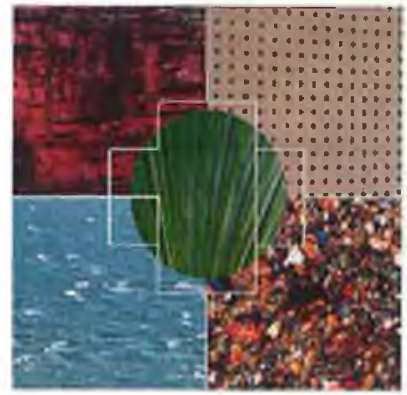

(g)

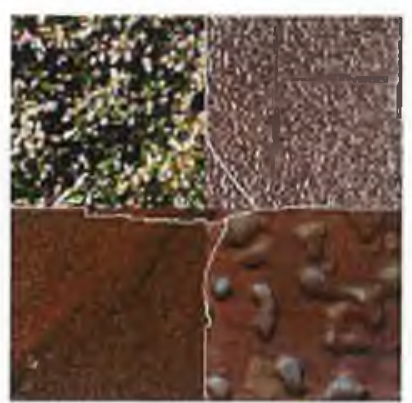

(b)

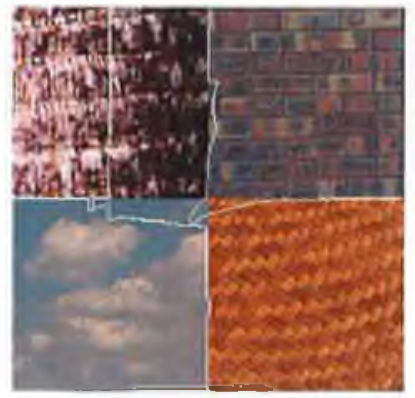

(d)

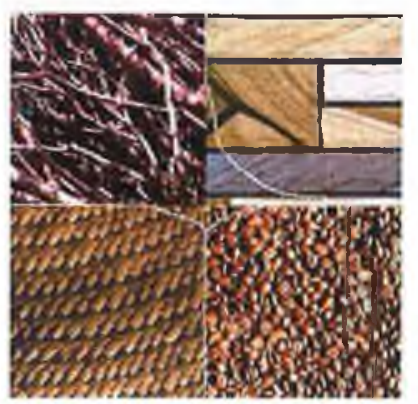

(f)

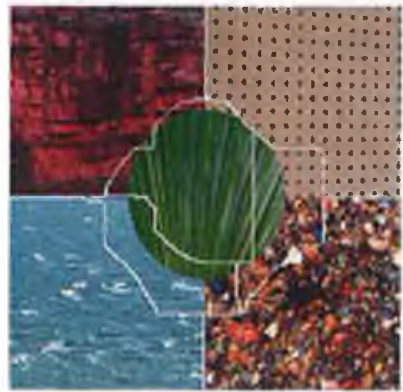

(h)

Figure 5.18: Sample results illustrates the poor performance of the pixelwise classification 


\subsection{Segmentation Results Using Method-III}

In this section, analysis of the segmented results obtained using $\mathrm{LBP} / \mathrm{C}$ with colour clustering and DCT with colour clustering is presented. The results presented were obtained from a sample of 15 mosaic images. The segmentation results on the complete set of images using MIII is presented in Figure G.1 Figure G.6 (see Appendix G). Results for each of the two colour texture feature extraction approaches using the unsupervised texture segmentation are detailed below.

\subsubsection{Performance Evaluation}

Various tests were performed to analyse the effectiveness of the approaches and also to test the effect of colour spaces. A set of three values each for split threshold and merge threshold was considered to determine the minimum segmentation error. The split threshold was tested for 1.1, 1.2 and 1.3 and merge threshold was tested for $0.8,0.9$ and 1.0. The best values for the split and merge thresholds

\begin{tabular}{|l|l|l|}
\hline Mosaics & $\begin{array}{l}\text { Error for } \\
\text { (Appendix }\end{array}$ & $\begin{array}{l}\text { Error for } \\
\text { Block Size } \\
\text { D) Size } \\
\text { D) }\end{array}$ \\
\hline M1 & 0.0305 & 3.5156 \\
M3 & 0.0076 & 1.9531 \\
M4 & 0.0091 & 1.9531 \\
M8 & 0.0061 & 3.125 \\
M9 & 0.0457 & 4.296 \\
\hline Average & 0.0198 & 2.9685 \\
\hline
\end{tabular}

Table 5.2: Quantitative evaluation for different block size

were found to be 1.2 and 0.9 respectively. Based on the segmentation results, the 


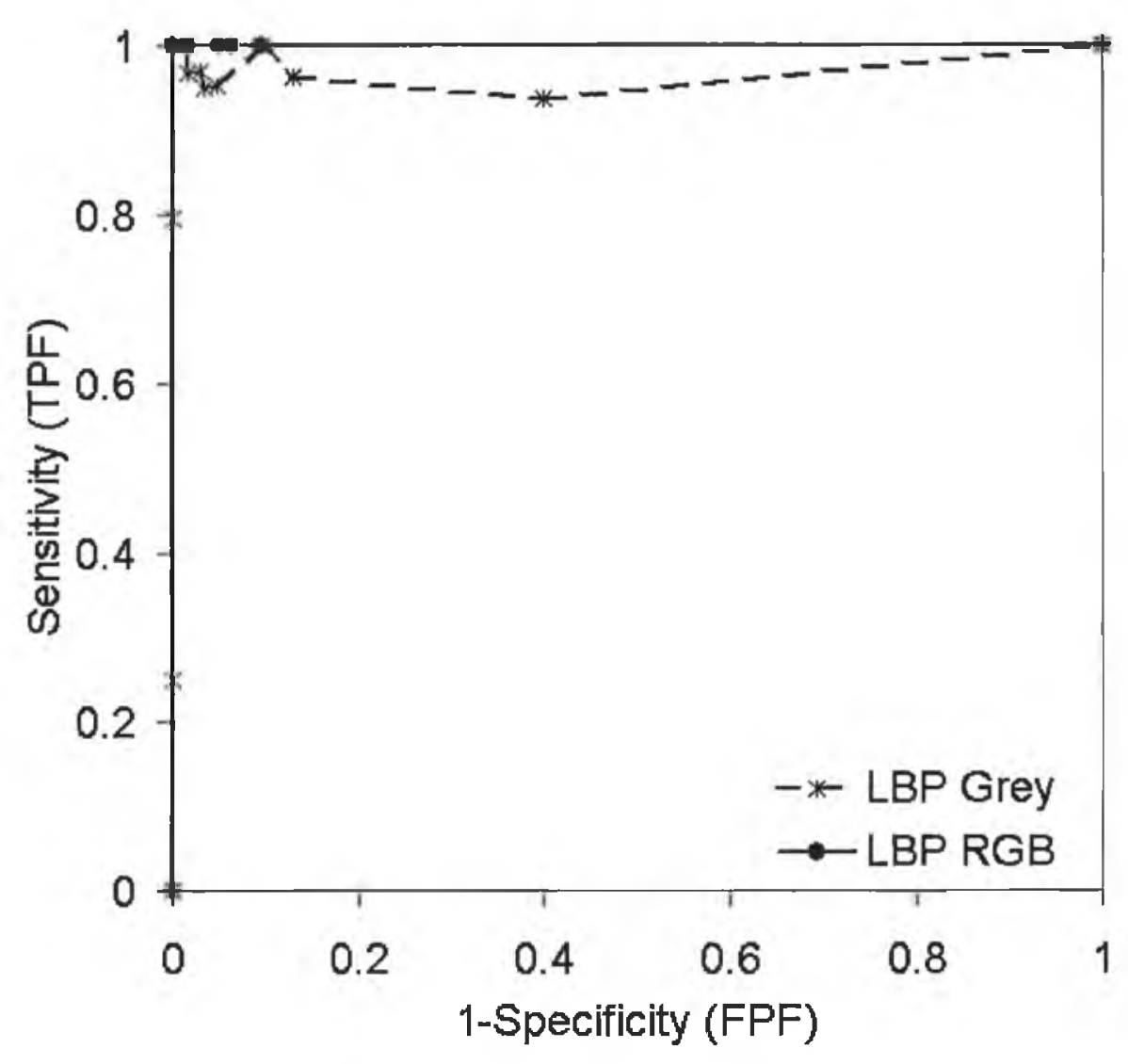

Figure 5.19: ROC curves for $\mathrm{LBP} / \mathrm{C}$ in greyscale and $\mathrm{LBP} / \mathrm{C}$ with colour clustering in RGB space

number of bins for contrast is quantised to 8 bins. Comparable results were obtained with 16 bins as well. This is in correspondence with Ojala et al. [21]. The block size until which the splitting should be performed was tested on a quantitative basis. Table 5.2 shows the quantitative evaluation of results obtained using a block size of 32 and a block size of 16 over the set of mosaic images. Summarising, an error mean of nearly less than $0.2 \%$ and nearly $3 \%$ has been obtained for the set of test images. Hence a block size of 32 was considered for the experiments. 


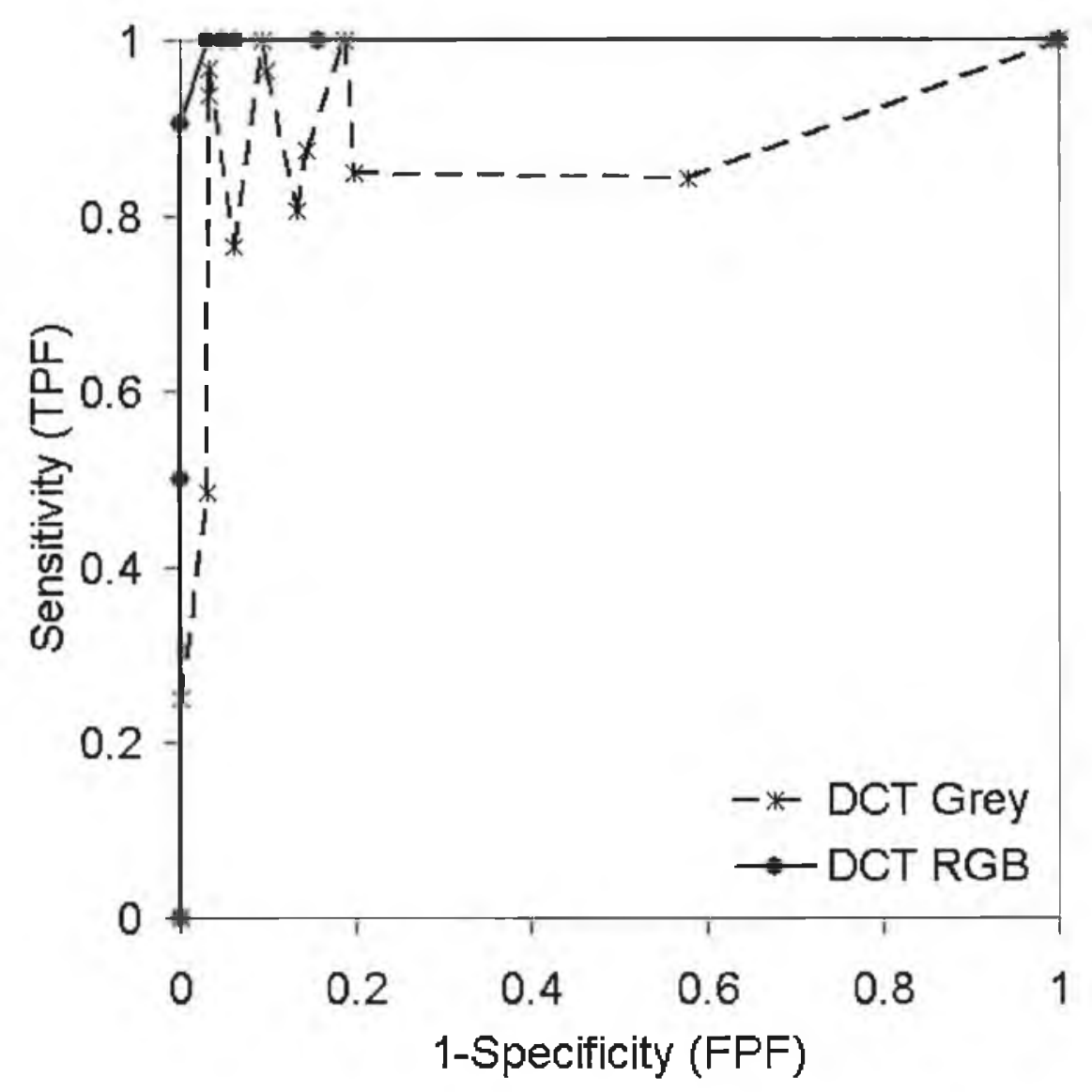

Figure 5.20: ROC curves for DCT in greyscale and DCT with colour clustering in RGB space

The first test is to evaluate the performance of the grey scale images and the colour images using Method-III. To illustrate this, the segmentation results for greyscale LBP/C or DCT, and LBP/C with colour clustering or DCT with colour clustering in RGB colour space were obtained. The true positive fraction against the false positive fraction was plotted in the ROC curve. The graph in Figure 5.19 and Figure 5.20 shows that the segmentation performance increases due to the inclusion of colour information. The result shows an increase in the percentage of proper segmentation for the RGB space in both LBP/C with colour 
clustering and DCT with colour clustering approaches compared to the LBP/C grey scale and DCT grey scale results.

\begin{tabular}{|l|l|l|l|}
\hline Technique & RGB & YIQ & HSI \\
\hline LBP & $95 \%$ & $96.6 \%$ & $95 \%$ \\
\hline DCT & $85.5 \%$ & $95 \%$ & $90 \%$ \\
\hline
\end{tabular}

Table 5.3: The performance of the LBP/C with colour clustering and DCT with colour clustering in RGB, YIQ and HSI spaces as percentage of segmented results

The overall performance of the segmentation is shown in table 5.3. This is also shown as a graphical plot in Figure 5.21 and Figure 5.22. On comparing the results, the relative merits of the different colour spaces were found to be nonconclusive.

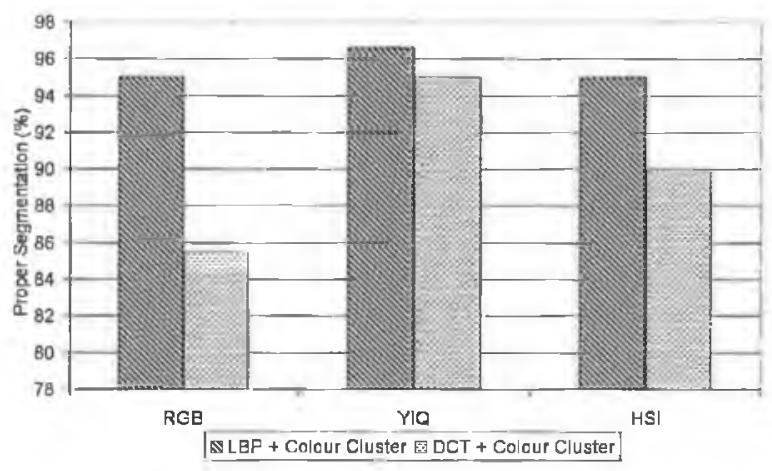

Figure 5.21: Bar chart representing the performance of the two approaches in RGB, YIQ and HSI colour space

However, from Figure 5.21 the YIQ colour space produced similar results using LBP/C with colour clustering and DCT with colour clustering. There is no significant difference in the segmentation results using the three colour spaces. This suggests that none of the colour spaces is superior. All the three colour spaces performed equally well. This is in accordance with the review performed in chapter 2 [14]. 
The following conclusions can be drawn from table 5.3 and Figure 5.22. The best performance was achieved by using LBP/C with colour clustering feature extraction technique for colour texture segmentation. When the texture feature extraction technique is changed from LBP to DCT, there is a considerable variation in the segmentation results.

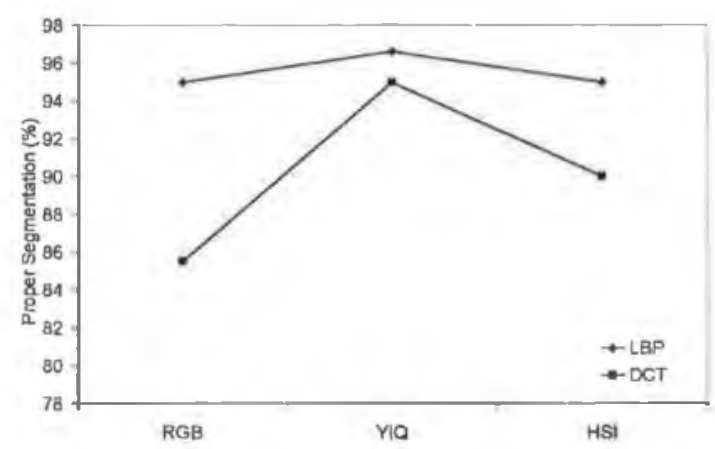

Figure 5.22: Comparison of different approaches in Method-III

A test was carried out to demonstrate the importance of the colour and the texture descriptors for colour texture segmentation. Table 5.4 and table 5.5 illustrates the segmentation error based on the colour and texture features. The parameter values for split threshold and merge threshold are set to 1.2 and 0.9 respectively. The error rate for each image was computed at the end of merging stage to compare the weights. The weights in the homogeneity measure were replaced with different values ranging from 0.0 to 1.0 at intervals of 0.2 . The error,

$$
e=\frac{\text { number of pixels incorrectly segmented in the image }}{\text { Total number of pixels in the segmented image }}
$$

The mean error rate for different colour and texture weights were calculated. The error rates in table 5.4 shows that up to a weight of 0.6 for texture the segmentation error is minimal, beyond which the error increased significantly. The error in the colour weights $1.0,0.8,0.6$ and 0.4 is mainly due to the colour variation in the images, these errors are acceptable. The errors at the texture 


\begin{tabular}{|l|l|l|}
\hline \multicolumn{2}{|c|}{ Weights } & \multirow{2}{*}{ હ (\%) } \\
\cline { 1 - 2 } Colour & $\begin{array}{l}\text { Texture } \\
(\mathrm{LBP})\end{array}$ & \\
\hline 1.0 & 0.0 & 4.84 \\
0.8 & 0.2 & 4.37 \\
0.6 & 0.4 & 4.53 \\
0.4 & 0.6 & 3.59 \\
0.2 & 0.8 & 11.71 \\
0.0 & 1.0 & 26.71 \\
\hline
\end{tabular}

Table 5.4: Average segmentation error (\%) depending on the colour and the LBP texture weights

\begin{tabular}{|l|l|l|}
\hline \multicolumn{2}{|c|}{ Weights } & \multirow{2}{*}{ e (\%) } \\
\cline { 1 - 2 } Colour & $\begin{array}{l}\text { Texture } \\
\text { (DCT) }\end{array}$ & \\
\hline 1.0 & 0.0 & 14.53 \\
0.8 & 0.2 & 9.06 \\
0.6 & 0.4 & 4.06 \\
0.4 & 0.6 & 11.56 \\
0.2 & 0.8 & 11.56 \\
0.0 & 1.0 & 39.37 \\
\hline
\end{tabular}

Table 5.5: Average segmentation error (\%) depending on the colour and the DCT texture weights

weights 0.8 and 1.0 were found to be substantial. Similarly table 5.5 presents the results for colour cluster weights and the DCT texture weights. The results presented imply that the segmentation error is minimum for the colour weight 0.6 and texture weight 0.4 . The exclusion of texture or colour weights resulted 
in an increase in segmentation error. From tables 5.4 and 5.5, it is apparent that colour weight 0.6 and texture weight 0.4 resulted in a good segmentation with minimum error. This is in agreement with the findings from Chen [73].

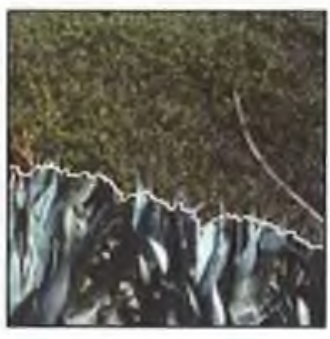

(a)

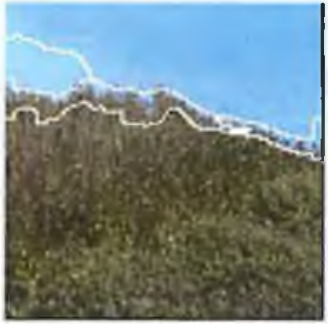

(d)

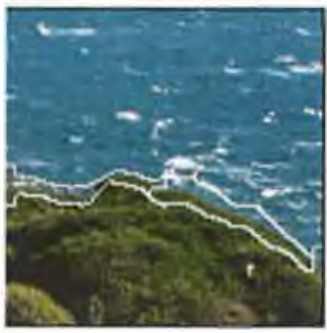

$(\mathrm{g})$

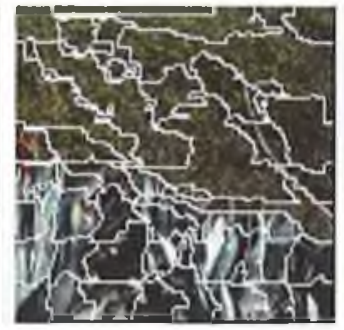

(b)

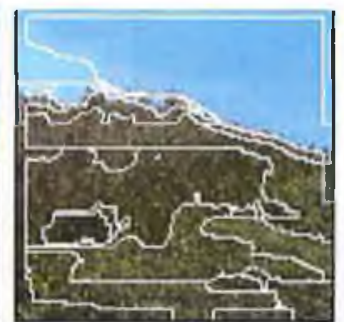

(e)

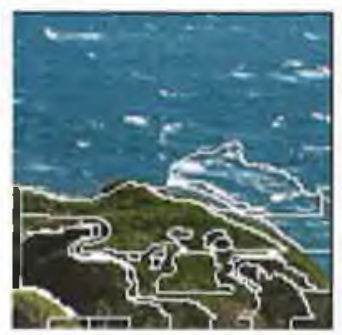

(h)

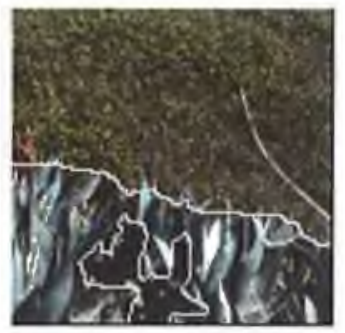

(c)

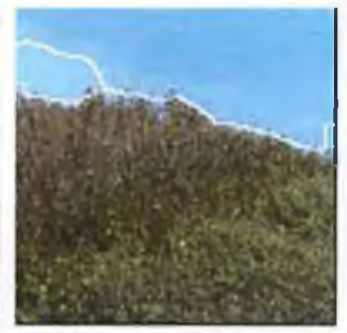

(f)

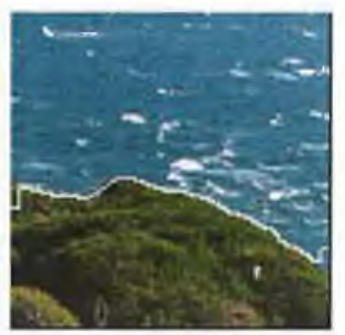

(i)

Figure 5.23: (a), (d), (g) Segmentation based on colour, (b), (e), (h) Segmentation based on texture, (c), (f), (i) Segmentation based on colour and texture

The automatic selection of colour weight and texture weight as presented in chapter 4 produces an error rate of less than 1.0\%. The proposed framework for colour texture segmentation selects the weights automatically. The results in Figure 5.23 shows that the error in segmentation based on manual selection of colour and texture weights is higher compared to the segmentation based 
on automatic selection of colour and texture weights. In addition, the results depicted in Figure 5.23 illustrate that the incorporation of colour with texture increases the accuracy of colour texture segmentation. The following conclusions can be drawn from these results: Texture alone or colour alone cannot provide a good segmentation. The proper inclusion of both colour and texture is necessary for a good colour texture segmentation. In some cases, the exclusion of texture weights provides a reasonable segmentation, but the exclusion of colour weights resulted in poor segmentation.

\subsubsection{Influence of Texture and Colour}

The boundaries obtained after the merging stage and the boundary refinement stage are presented in Figure 5.24 and Figure 5.25. The sketches show the detected border embedded over the original image. Colour texture segmentation results shown in these figures used LBP/C with colour clustering features. Meaningful regions in images were successfully detected and the usefulness of the proposed colour texture segmentation method was demonstrated. In addition, few aspects of the results which were considered to be important were emphasised.

The first image Figure 5.24-MII-LBP-1a has dark and light blue colours in water in the third and fourth quadrants. This was well separated by the colour properties. The leaf in Figure 5.24-MIII-LBP-2a shows a white patchy region, which was identified as a separate region. The result in Figure 5.24-MIII-LBP-3a and Figure 5.24-MIII-LBP-4a at the end of merging stage may seem to be an inaccurate segmentation. But the result after boundary refinement shows the accuracy with which segmentation is performed. Though the result is not equivalent to the ground truth (considering the ground truth in a macro scale which represents four quadrants), as per the human knowledge this segmentation is 

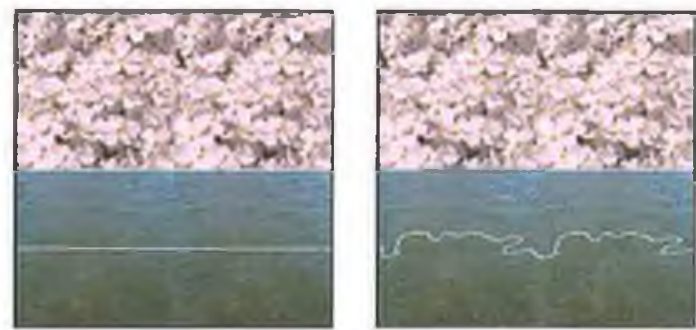

(MIII-LBP-1a)

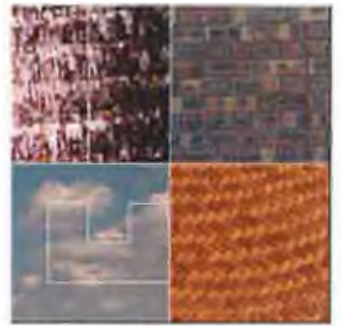

(MIII-LBP-3a)

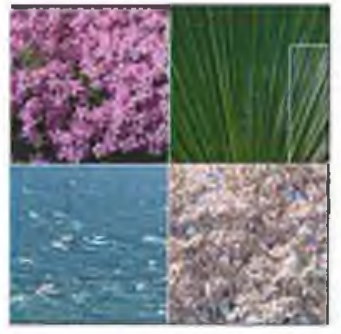

(MIII-LBP-5a)

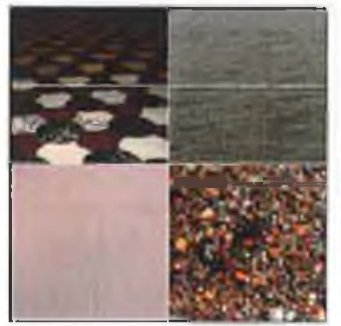

(MIII-LBP-7a)
(MIII-LBP-1b)

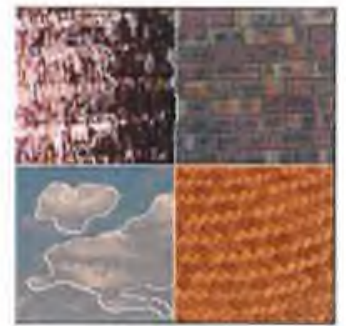

(MIII-LBP-3b)

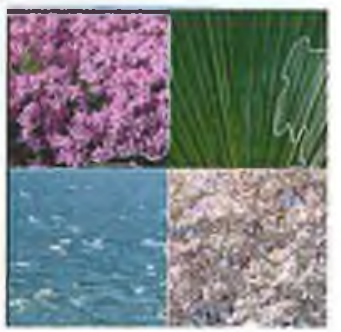

(MIII-LBP-5b)

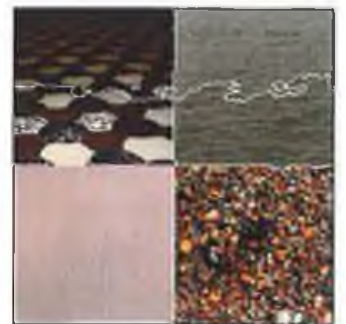

(MIII-LBP-7b)
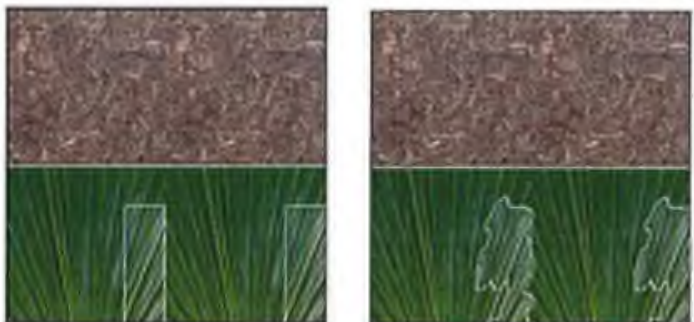

(MIII-LBP-2a)

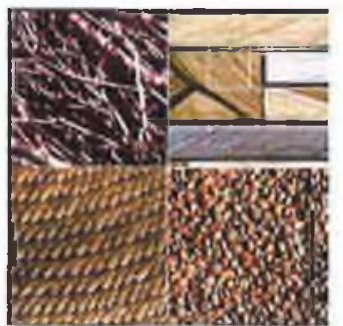

(MIII-LBP-4a)

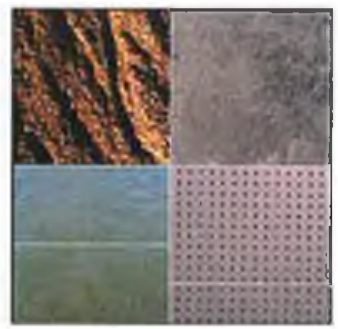

(MIII-LBP-6a)

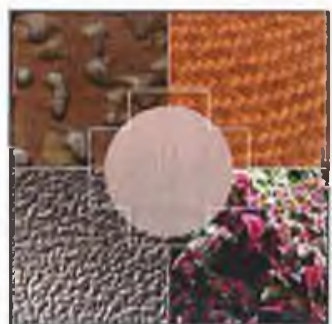

(MIII-LBP-8a)
(MIII-LBP-2b)

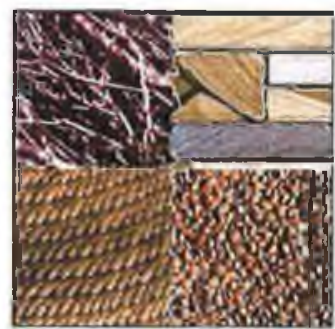

(MIII-LBP-4b)

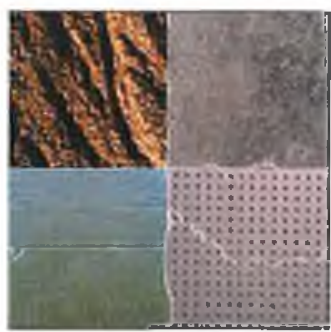

(MIII-LBP-6b)

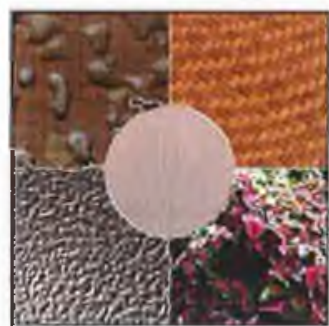

(MIII-LBP-8b)

Figure 5.24: Sample segmented results from Method-III using LBP with colour clustering 


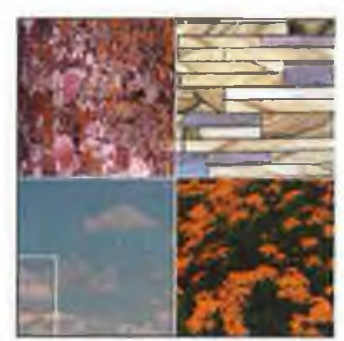

(MIII-LBP-9a)

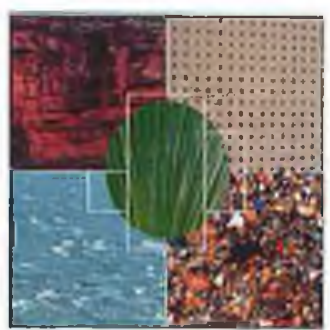

(MIII-LBP-11a)

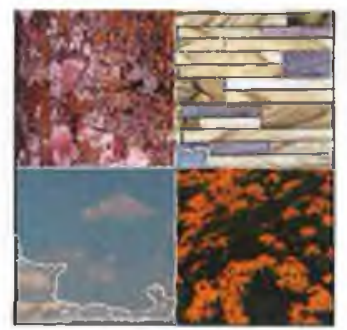

(MIII-LBP-9b)

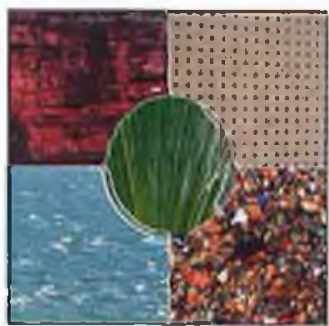

(MIII-LBP-11b)

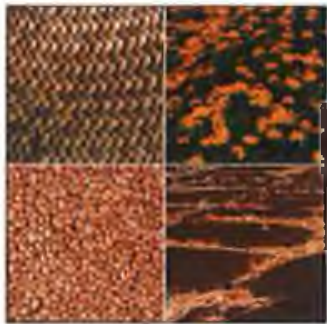

(MIII-LBP-10a)

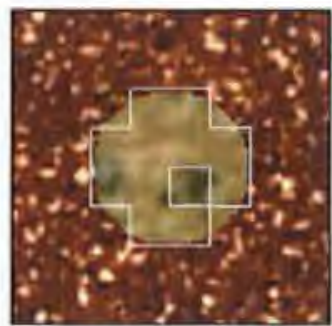

(MIII-LBP-12a)

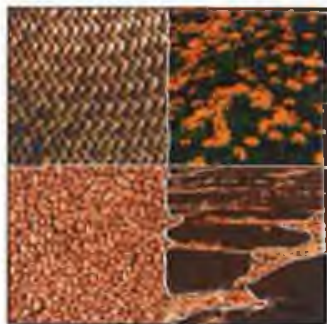

(MIII-LBP-10b)

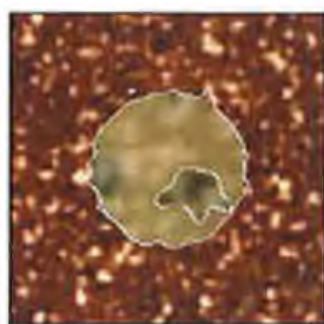

(MIII-LBP-12b)

Figure 5.25: Sample segmented results from Method-III using LBP with colour clustering

perfect. Similarly, Figure 5.24-MIII-LBP-5a and Figure 5.24-MIII-LBP-6a produced anticipated results in accordance to human perception. The segmentation in Figure 5.24-MIII-LBP-7a and Figure 5.24-MIII-LBP-8a are an example for the colour texture segmentation based on the colour texture variations.

Figure 5.25-MIII-LBP-9a shows that the region in the third quadrant representing clouds was incorrectly segmented. But the boundary refinement enhances the segmentation by properly identifying the borders of the clouds. Figure 5.25-MIIILBP-10a represents a good segmentation and is in agreement with the ground truth. But the boundary refinement which is based on the colour clustering alone could not distinguish the different textures which are of the same colour. These type of errors were rarely observed. In few cases, such as Figure 5.25-MIII-LBP11a the error recovery after merging is possible during the boundary refinement. Figure 5.25-MIII-LBP-12a represents a segmentation with a small block inside 
the circle which is properly segmented and refined in the final process.

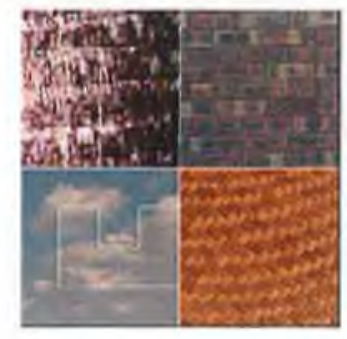

(MIII-DCT-1a)

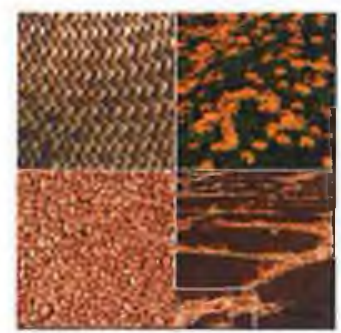

(MIII-DCT-3a)

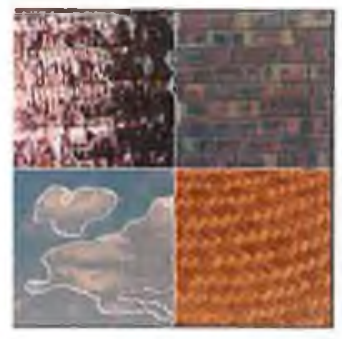

(MIII-DCT-1b)

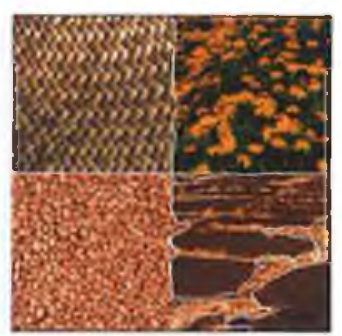

(MIII-DCT-3b)

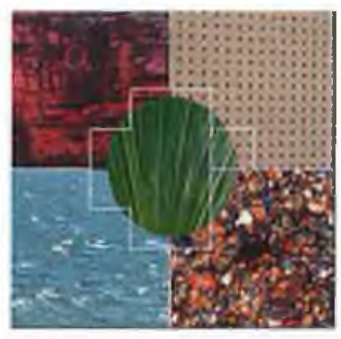

(MIII-DCT-2a)

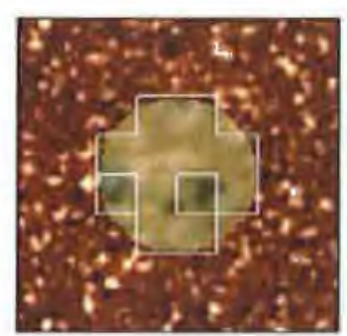

(MIII-DCT-4a)

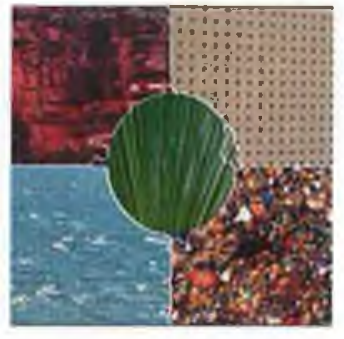

(MIII-DCT-2b)

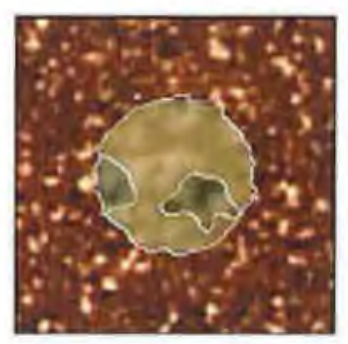

(MIII-DCT-4b)

Figure 5.26: Sample segmented results from Method-III using DCT with colour clustering

Sample segmentation results from the DCT with colour clustering features are presented in Figure 5.26. Figure 5.26-MIII-DCT-1a and Figure 5.26-MIII-DCT2 a represents some segmentation error in the merging stage. Similar to the previous approach using LBP/C with colour clustering these errors were recovered and the clouds in the third quadrant and the center circle were properly segmented. The image in Figure 5.26-MIII-DCT-3a represents some small unsegmented region. As previously stated, this was not refined in the boundary refinement as the different textures with the same colour were not distinguished. Figure 5.26-MIII-DCT-4a represents an image with two small regions inside the circle according to the difference in the colour. Depending on the colour space the region is segmented accurately. 


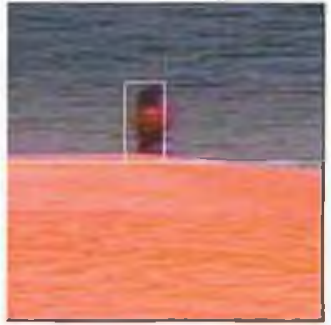

(MIII-LBP-N1a)

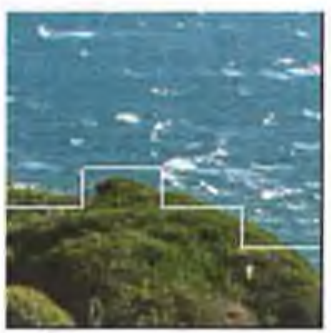

(MIII-LBP-N3a)

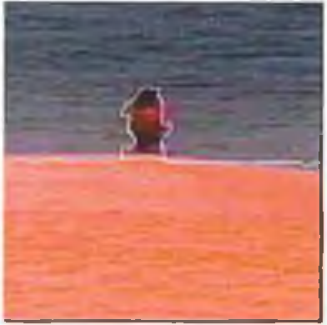

(MIII-LBP-N1b)

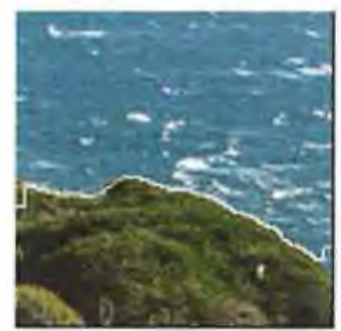

(MIII-LBP-N3b)

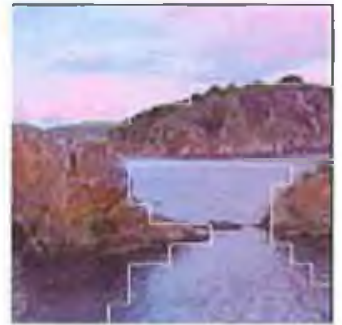

(MIII-LBP-N2a)

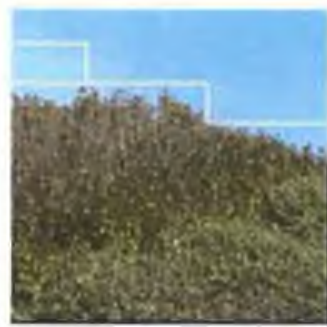

(MIII-LBP-N4a)

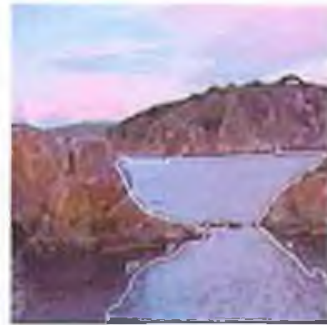

(MIII-LBP-N2b)

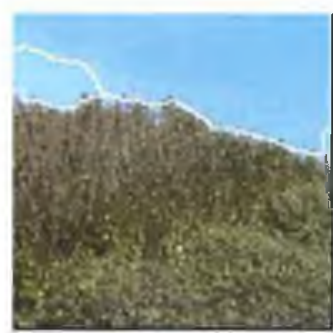

(MIII-LBP-N4b)

Figure 5.27: Segmented results of natural images from Method-III using LBP/C with colour clustering

The performance of the proposed method for colour texture segmentation was tested over a set of 10 real images of which 4 sample images are shown in Figure 5.27. Natural images have the rich source of colour and textures. Segmentation of natural images is more complex, since defining the boundaries between various regions is difficult. The parameters used for the natural images differed from those of the mosaic images. For most images the split threshold value is 1.1 and the merge threshold value is 1.0 .

Figure 5.27-MIII-LBP-N1a represents the segmented beach image and the refined image. As stated before, the segmentation merged the small features within the bird as a single region. Figure 5.27-MIII-LBP-N2a shows the segmented image of the rocks and sea. The clouds in the region is considered as a single region. In addition, the regions on the rock and sea were merged together as a single 
region. In few cases different textures with similar colour were identified as same region. This is due to the influence of the colour and the proposed algorithm was not able to differentiate as that of the human beings. Figure 5.27-MIII-LBP-N3a shows a good segmentation. Figure 5.27-MIII-LBP-N4a result demonstrates the segmentation of the clouds in the sky region. More results on natural images are presented in Appendix G. The segmentation of the natural images are acceptable.

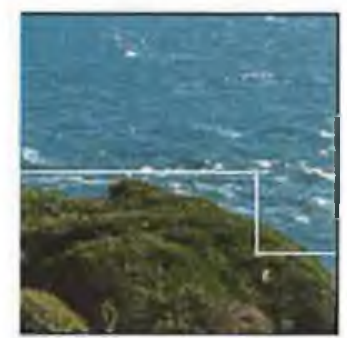

(MIII-DCT-N1a)

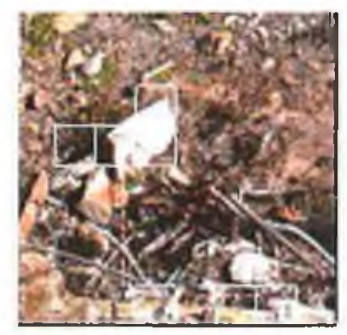

(MIII-DCT-N3a)

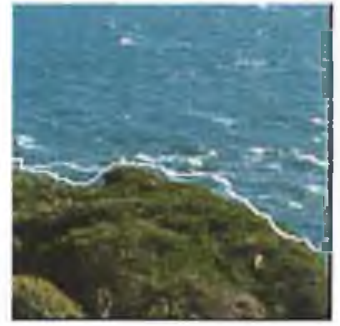

(MIII-DCT-N1b)

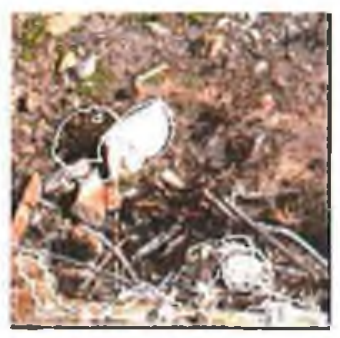

(MIII-DCT-N3b)

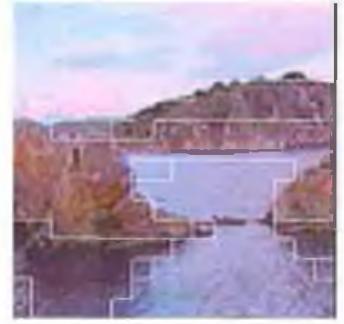

(MIII-DCT-N2a)

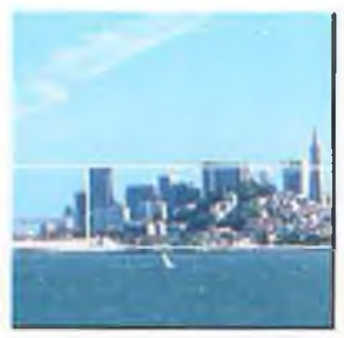

(MIII-DCT-N4a)

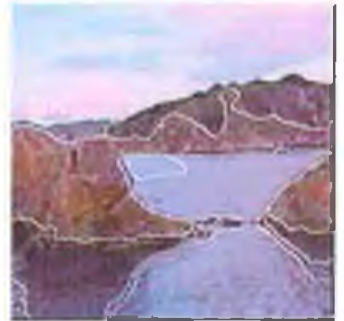

(MIII-DCT-N2b)

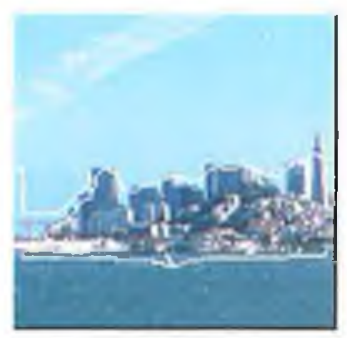

(MIII-DCT-N4b)

Figure 5.28: Segmented results of natural images from Method-III using DCT with colour clustering

Figure 5.28 shows the segmentation results of natural images using MethodIII (DCT with colour clustering). Figure 5.28-MIII-DCT-N1b represents the segmentation result after boundary refinement. The segmentation result after merging stage was not proper, but the boundary refinement algorithm fine-tunes the borders between the rock and the sea. Figure 5.28-MIII-DCT-N2a illustrates the segmentation of the rock and the sea image. The clouds in the sky were not 
segmented separately. The whole sky was considered as a single region. Unlike the segmentation using LBP with colour clustering (see Figure 5.27-MIII-LBP$\mathrm{N} 2 \mathrm{a}$ ), the region between the rock and the sea was split properly though the colour components appear the same. The rocks should be segmented as a single entity; however based on the difference in colour and texture this is split into a different regions. In Figure 5.28-MIII-DCT-N3a, due to the presence of large number of small particles, the image could not be segmented properly. Figure 5.28-MIII-DCT-N4a shows a group of buildings on the shore of a sea. In the merging stage the boat was not segmented properly, after the boundary refinement stage the boat was separated from the sea and merged with the group of buildings. The clouds were considered together with the sky as a single region. Overall the segmentation results obtained were acceptable.

The use of adaptive smoothing is illustrated in Figure 5.29. This is used as a preprocessing, before clustering the colour features, to eliminate noise and to increase the cluster continuity. This smoothing has the advantage to protect the boundaries from blurring. Figure $5.29[\mathrm{a}$, e] represents the smoothed and the clustered image. Figure 5.29 [b, f] shows the merged image and Figure 5.29 [c, g] shows the boundary refined image after smoothing. Figure $5.29[\mathrm{~d}, \mathrm{~h}]$ shows the boundary refined result of the same image without adaptive smoothing. A comparison of the results with and without smoothing shows the effect of the adaptive smoothing. Figure 5.29 (c) and (d) shows the boundary refined segmented result with and without smoothing respectively. The result demonstrates that the segmentation is erroneous and the green grass is wrongly segmented without smoothing. But the smoothing removes the noise and improves the segmentation in the boundaries between two different grass regions. Also, Figure $5.29(\mathrm{~g})$ and (h) illustrates that the segmentation without smoothing could not identify the boundaries properly. The smoothing correctly recognises the boundaries which are evident from the clustered image by the thin blue line between the sky and 


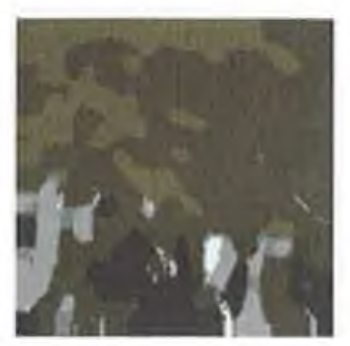

(a)

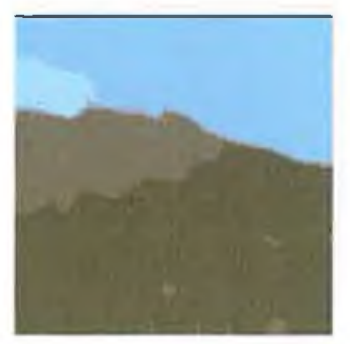

(e)

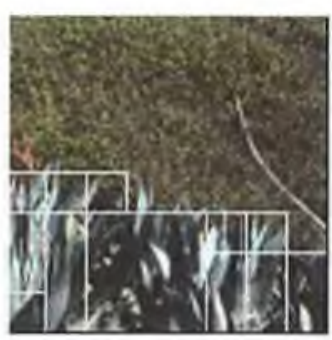

(b)

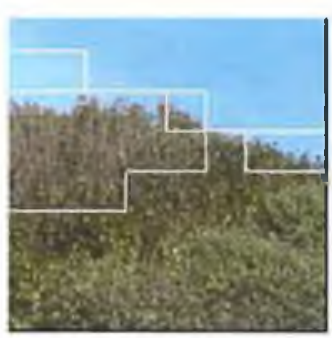

(f)

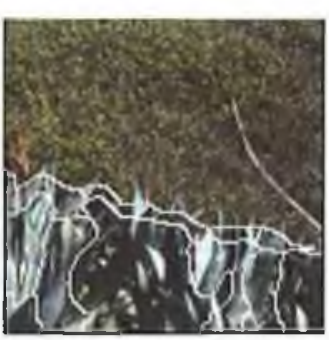

(c)

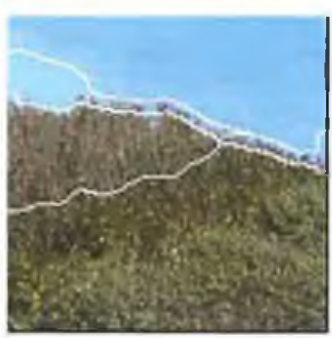

(g)

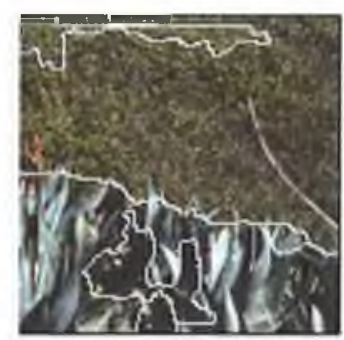

(d)

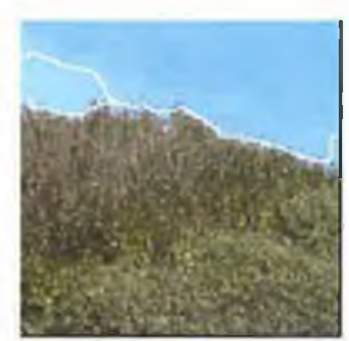

(h)

Figure 5.29: The influence of adaptive smoothing

the plants, and in addition a small region of the plant region was also segmented properly. This shows that the adaptive smoothing is mainly useful for the images with irregular boundaries such as natural images, this prevents over segmentation and helps to obtain a better segmentation on the boundaries.

To examine the integrity of the developed colour texture methodology, the sample image database was subjected to a test procedure. Typical test procedure included introduction of noise in the image followed by the application of colour texture methodology. If the resulting segmented image remained similar to the results obtained in image without the noise, it can be concluded that the developed colour texture methodology is robust. A random noise of $1 \%, 2 \%$ and $4 \%$ is introduced in the image and the proposed colour texture segmentation method is applied on these noisy images. From the resulting images, it is evident that for noise levels of $1 \%$ and $2 \%$ the segmented results remained similar to those of the image without noise. 


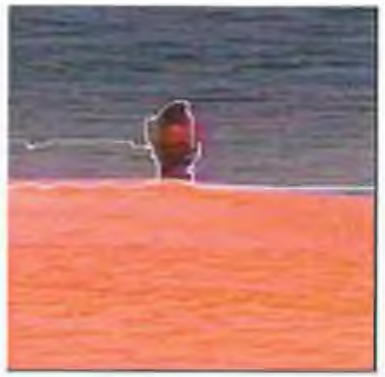

(1a)

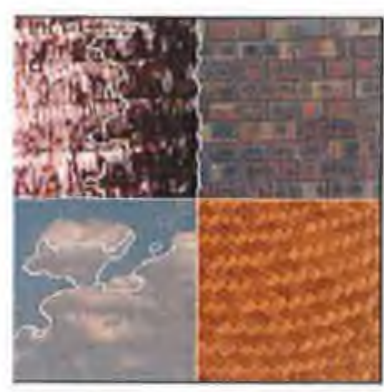

(2a)

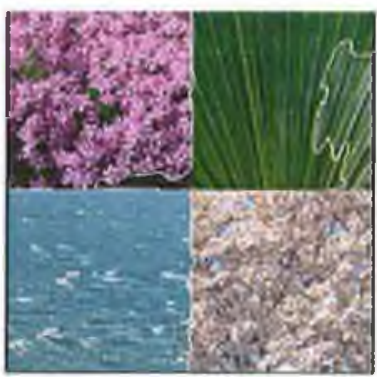

(3a)

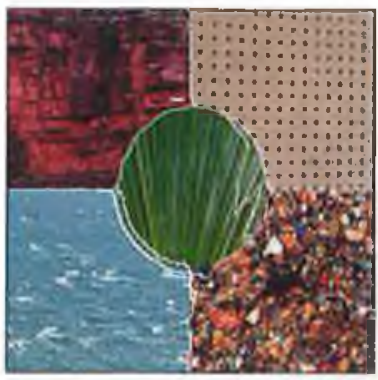

(4a)

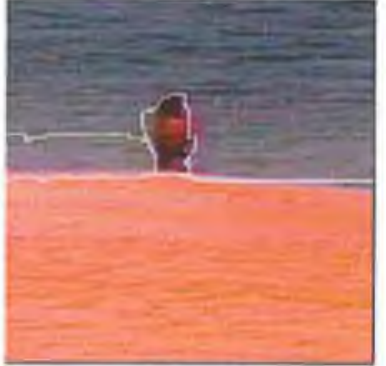

(1b)

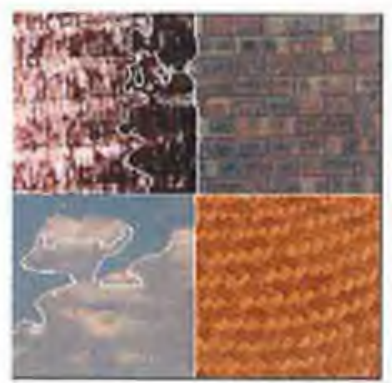

$(2 \mathrm{~b})$

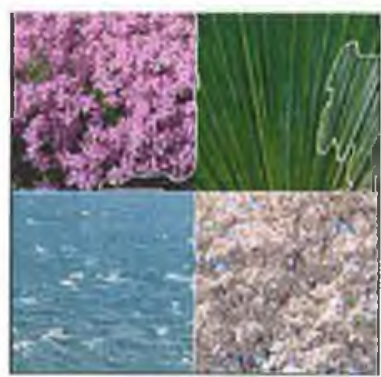

(3b)

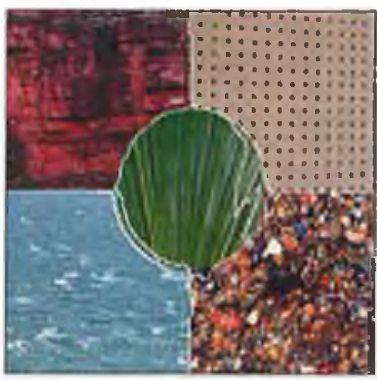

(4b)

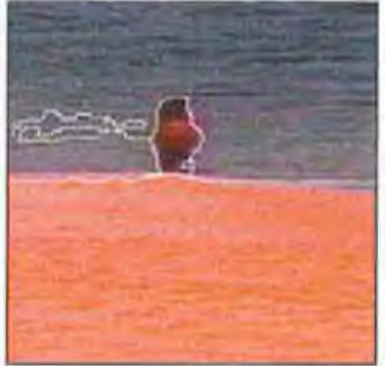

(1c)

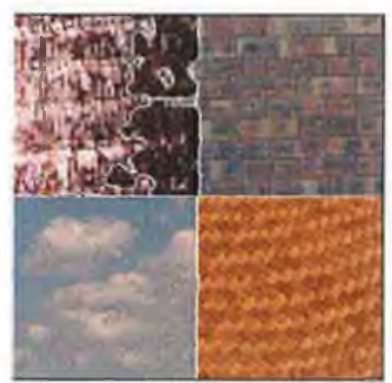

$(2 c)$

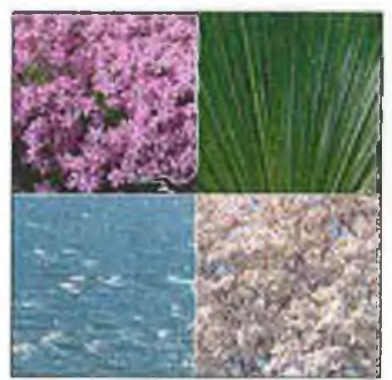

(3c)

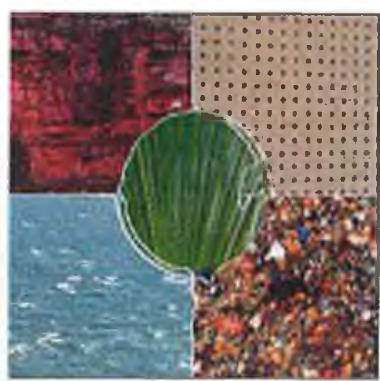

$(4 \mathrm{c})$

Figure 5.30: The influence of noise, $\mathrm{a}-1 \%, \mathrm{~b}-2 \%, \mathrm{c}-4 \%$, in the colour texture segmentation 


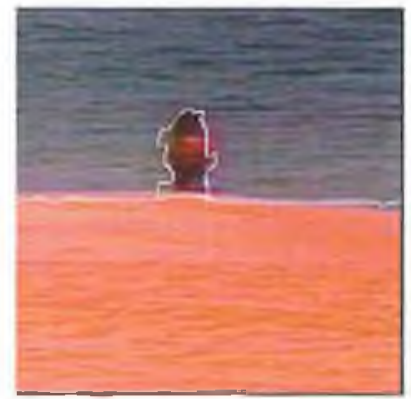

(a)

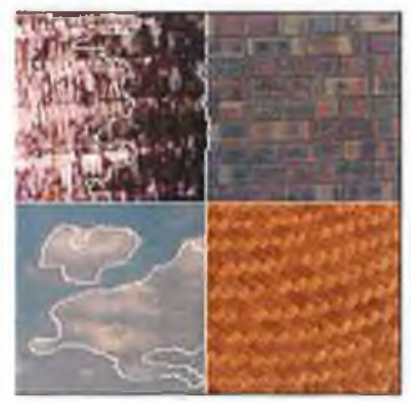

(c)

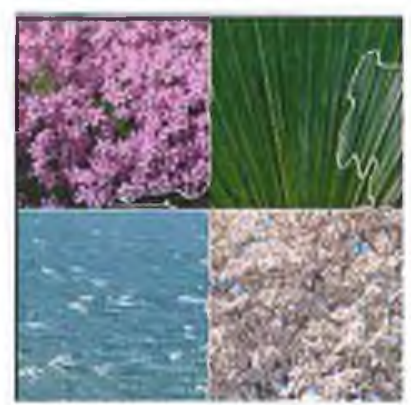

(e)

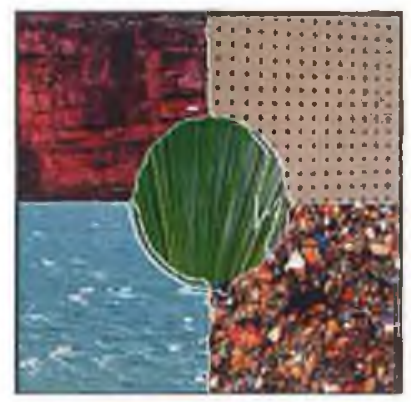

(g)

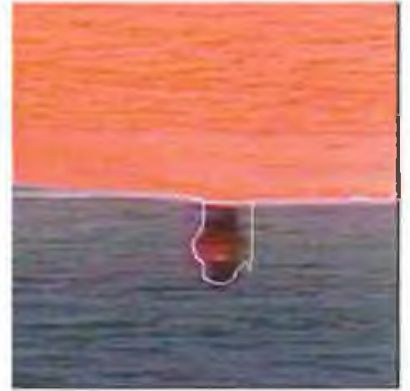

(b)

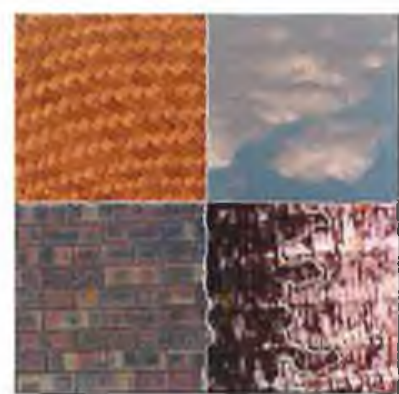

(d)

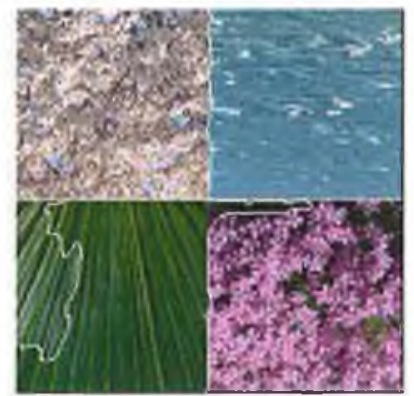

(f)

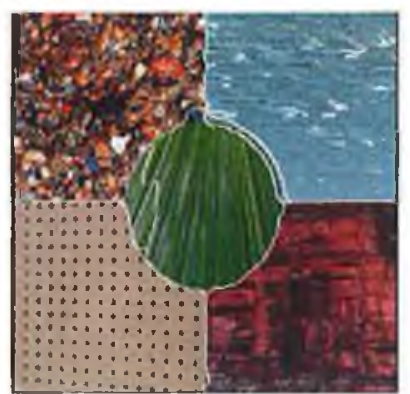

(h)

Figure 5.31: Segmentation effect of the rotated images 
On generating $4 \%$ noise in the image, noticeable difference in the segmented result is evident. This clearly shows that the developed methodology takes into account the variations in colour and texture present in the image. Figure 5.30 illustrates the effect of noise for various percentages.

In addition to the above test procedure, the proposed method was tested for rotation invariance. The images were rotated to $180^{\circ}$ and colour texture segmentation method was applied. It can be observed from the resulting segmented images that the developed colour texture segmentation methodology is rotation invariant. Similar segmented results were obtained after rotation of the image, with few exceptions (see Figure 5.31 (c) and (d)). In this image, the clouds were identified as a separate region in the original image, whereas the clouds were merged with the sky in the rotated image. This may be due to the non-rotation invariant property of LBP.

The disadvantage of the method is that the method fails in situations such as regions with similar colour with different textures. This is due to the greater impact of the colour and the use of colour histograms for the boundary refinement. In Method-III, colour texture is described by LBP/C or DCT with colour clustering features. This method uses the histograms of colour clustering and the histograms of LBP/C or DCT to derive the similarity measure. This method also uses adaptive smoothing, in order to avoid over segmentation and to obtain a better segmentation in the boundaries. The boundary refinement based on the colour clustering histograms improves the boundaries and also helps to overcome errors from the merging stage. Many of the small components were properly separated and the segmentation was found to be appropriate. Hence the colour texture segmentation based on the Method-III is demonstrated to be efficient and reliable. 


\subsection{Discussion}

Before discussing in detail about the evaluation of the three different methods, this research work emphasises that the colour is an important feature and it forms the major contribution to the proposed methods. Colour not only improves the segmentation results but is useful in error recovery as well. In addition, the combination of colour texture properties improves the segmentation. In colour texture segmentation, colour or texture alone does not provide enough information for proper segmentation. Also, individual characteristics such as colour or texture does not best describe the colour texture analysis. Hence the combination of the colour and texture is essential to properly discriminate the colour texture regions. The correct description of the combination is necessary. The description of the colour textures were based on the colour and texture distributions in Method-III. This formed an efficient way to discriminate the colour texture regions. A detailed description about the three different methods used and the evaluation of the methods is given below.

The analyses were carried out to determine the usefulness of each of the three methods. Two earlier methods were implemented in this study for comparison purposes. Method-I used the texture segmentation as performed by Ojala et al. [21]. But this method cannot be included for comparison purpose as this method does not have an explicit use of colour features. Hence comparison of Method-I with the other methods was not carried out. Method-I was developed for preliminary studies.

Method-II uses the texture distributions together with the statistical colour features. The graph from Figure 5.32 illustrates the comparison between the best approaches from Method-II and Method-III. The LBP/C with colour features from Method-II is compared with LBP/C with colour clustering from MethodIII. Figure 5.32 shows that the best performer is the LBP/C with colour cluster- 


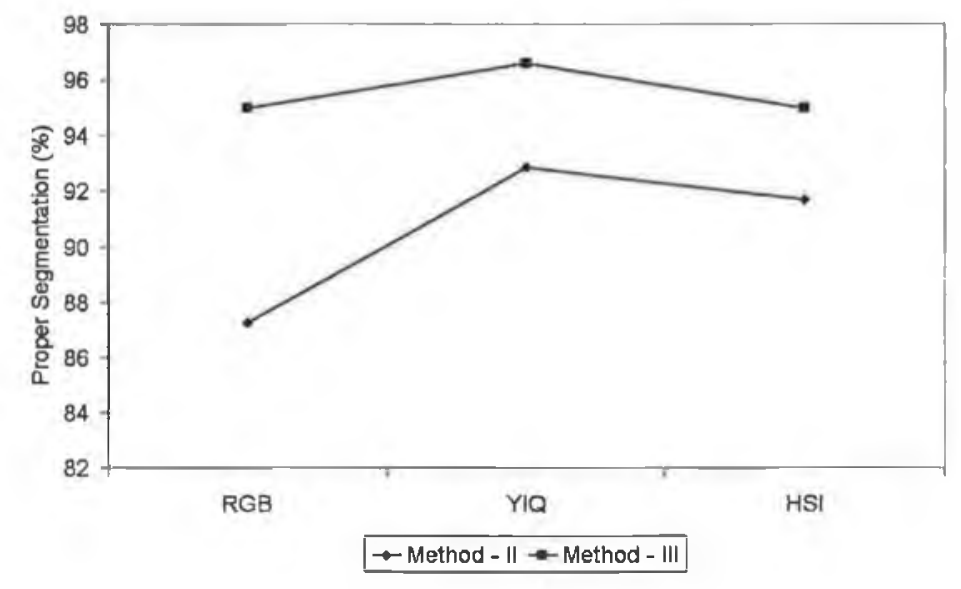

Figure 5.32: Comparison of Method-II using LBP/C with colour features and Method-III using LBP/C with colour clustering

ing. Hence Method-III is the best performer. Most of the regions were segmented properly using Method-III. The incorrectly segmented regions in Method-III were recovered by using the boundary refinement. The improper segmentation was not overcome in Method-II. Also the colour texture segmentation on images using Method-III provided a segmentation taking small colour difference in consideration. Colour texture segmentation is difficult and errors are often produced at boundaries. Error recovery is possible by using a boundary refinement algorithm in Method-III, which is an added advantage of the method. Hence, the accuracy of segmentation is not only based on the correct detection of regions but also the definite location of the boundaries between the regions. Hence, comparatively Method-III accurately segments the different blocks of the regions in an image. The algorithm works robustly and detected the abnormalities accurately.

Finally a comparison with other methods such as the colour texture segmentation method by Chen et al. [73] which uses colour distributions and local edge pattern distributions and segments the semantic regions such as the whole sky instead of the clouds which are not prominently identified. Method-III had a 
similar objective to that of the method by Mirmehdi and Petrou [86] to obtain a colour texture segmentation. It is hard to quantitatively review the best performer. This study can conclude that the regions are very well identified by Method-III and hence finally, this discussion concludes Method-III as the best performer.

\subsection{Algorithm Implementation}

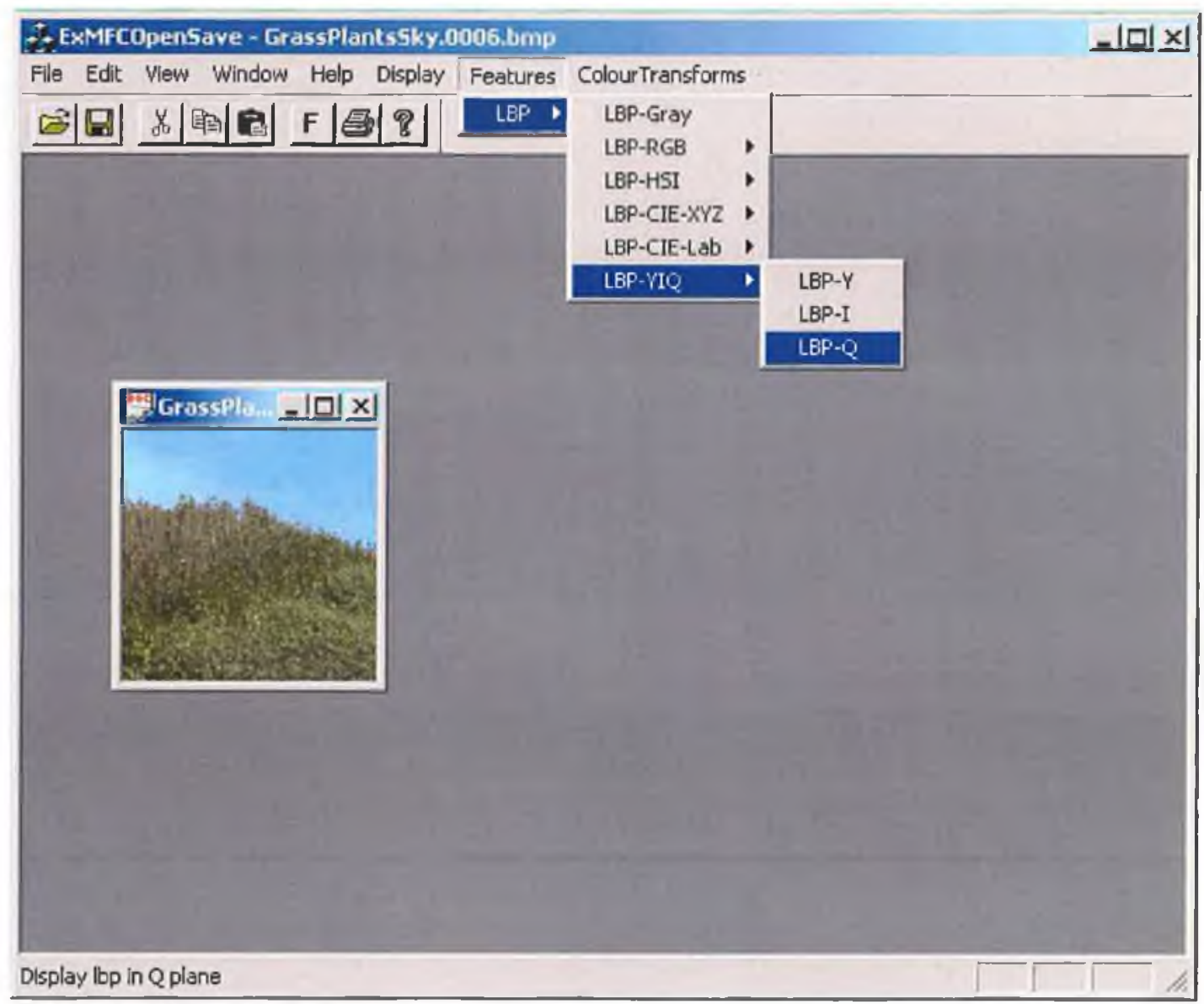

Figure 5.33: The executable file for Method-I

The proposed methods have been implemented on a personal computer with Intel Pentium III processor with 256MB RAM and $1.6 \mathrm{GHz}$ clock speed. The 
operating system used is Microsoft Windows 2000. The program was developed using Microsoft Visual $\mathrm{C}++6.0$ using Microsoft Foundation Class API. The windows bitmap file format is used for image input/output. Various generic and derived classes were created for the colour texture segmentation. The details of the classes used for the colour texture segmentation are given in Appendix C.

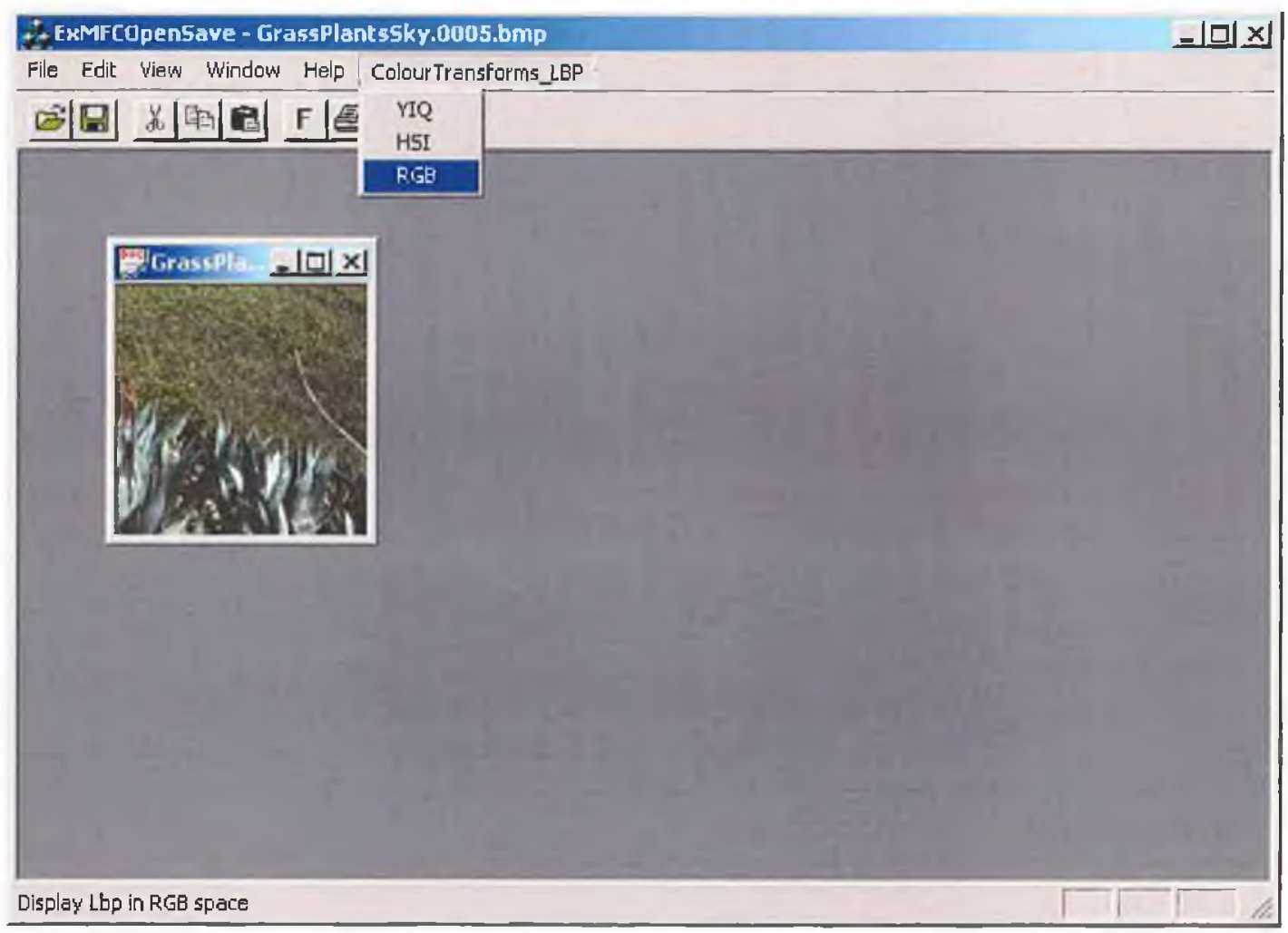

Figure 5.34: The executable file for Method-II using LBP/C

The average processing time for a $256 \times 256$ image in Method-I is 1.245 seconds with a scatter of $3 \%$. The speed of processing does not vary with planes, from which the segmentation was obtained. The average computational time for splitting the image is 0.5735 seconds and merging the image is 0.9 seconds. Irrespective of the different colour features and the colour spaces the processing time for Method-II remained the same. The average processing time for splitting 
using LBP and colour features is 0.879 seconds and merging is 0.6065 seconds and a total time taken for segmentation is 1.4855 seconds. The average processing time for splitting using DCT and colour features is 1.19 seconds and merging is 0.645 seconds.

The average time taken for unsupervised colour clustering is 14.611 seconds. The average time taken for splitting and merging is 1.442 and 1.6286 seconds respectively. The time taken to perform boundary refinement depends on the segmented result from the merging stage and also depends on the number of iterations it takes to complete the process. The adaptive smoothing takes 73.45 seconds. Overall, on an average Method-III took 17.68 seconds excluding adaptive smoothing and boundary refinement to perform colour texture segmentation. Comparatively, colour texture segmentation was carried out efficiently using Method-III. The execution window for Method-I, Method-II and Method-III is shown in Figure 5.33, Figure 5.34, Figure 5.35 and Figure 5.36 respectively. 


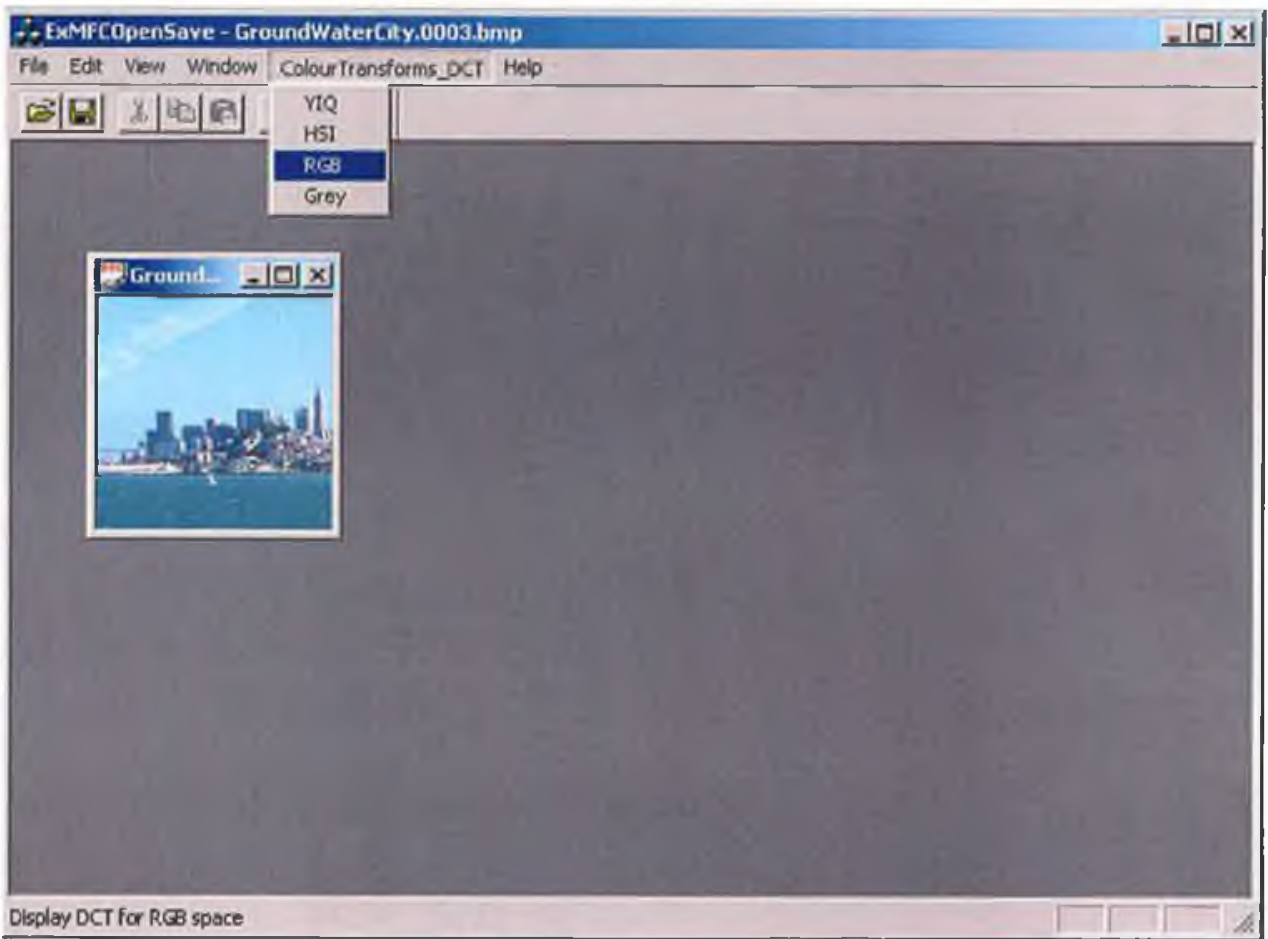

Figure 5.35: The executable file for Method-II using DCT

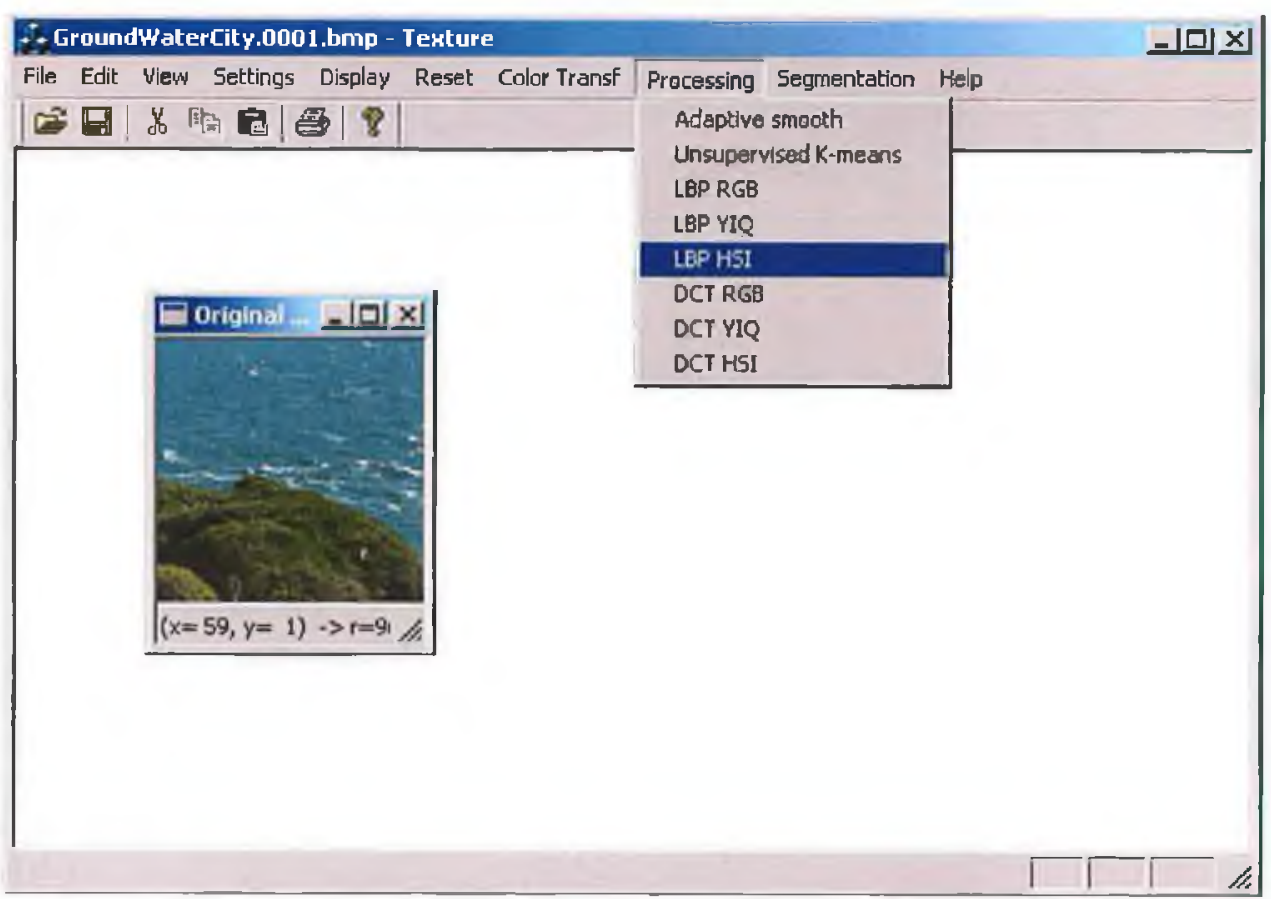

Figure 5.36: The executable file for Method-III 


\subsection{Summary}

This chapter tested the performance of the three methods and discussed the advantages and the drawbacks of the methods. Method-I was performed in the initial stages of this research, the results illustrate that the segmentation on the individual planes depends on the colour components in that particular plane. Method-II combined the distributions of texture and the colour features. This method performed well. But the refinement of the boundaries was not possible. Hence an effective and efficient way of combining colour and texture resulted in the development of Method-III. The use of distributions of the texture features together with distributions of the colour clustering in the homogeneity measure provided a good integration of colour and texture features. Also, error recovery was possible in this method using a boundary refinement algorithm. Hence Method-III was declared as the best performer. 


\section{Chapter 6}

\section{Application of the Developed Colour Texture Segmentation Methodology}

\subsection{Introduction}

This chapter presents the application of the proposed colour texture segmentation methodology. Among the three methods developed for colour texture segmentation in this study, better performance was established using Method-III. The developed framework can be applied to a number of different applications. Three out of a number of applications were selected and presented in this research work. The applications for colour texture segmentation include the segmentation of colour textured regions in Irish manuscript document, disease detection and the segmentation of underwater images. Application database consists of the Irish Script On Screen (ISOS) images, skin cancer images and the Sediment Profile Imagery (SPI). The following section presents the segmented results and the salient features of the segmentation are discussed. 


\subsection{Colour Texture Segmentation Applications}

\subsubsection{Irish Script on Screen Images}

The ISOS images are digital images of Irish manuscripts. The sample images were taken from old Irish manuscripts online - Irish Script On Screen (See: http://www.isos.dcu.ie/). About 5,000 early Irish language manuscripts survive and those appearing on this website form an important and distinctive part of Irish heritage. This includes the Book of Leinster, one of the language's most important manuscripts, compiled in the second half of the 12th century. The storage of letters, papers or other documents in ordinary stationery-grade folders or plastic sleeves invites certain deterioration even when kept in sealed containers. Slowly but inexorably, paper degrades and discolors, and ink fades. High heat and humidity are also detrimental. Most paper contains acid which over time will cause the paper to weaken and become brittle. A historic document which would otherwise appreciate greatly as a prime investment is debased in value. In addition, the corrosive effects of modern environment and time is a threat to document preservation. The objective of ISOS is to create digital images of Irish manuscripts, and to make these images available together with relevant commentary, accessible on a website. The purpose of such a website is to provide an electronic resource which will:

- provide exposure on the internet for a vital part of Ireland's cultural heritage.

- place these primary materials at the disposal of scholars and students.

- contribute to the conservation of these valuable books and documents by creating images of high-resolution detail which, generally speaking, will reduce the need to handle the artefacts themselves. 


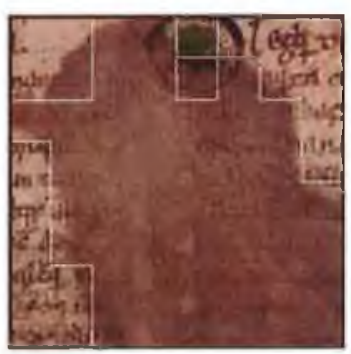

(1a)

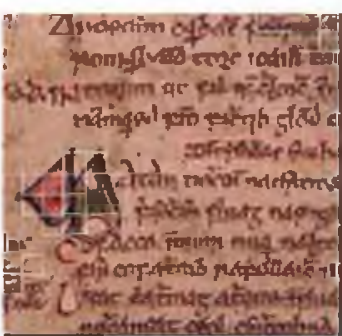

(3a)

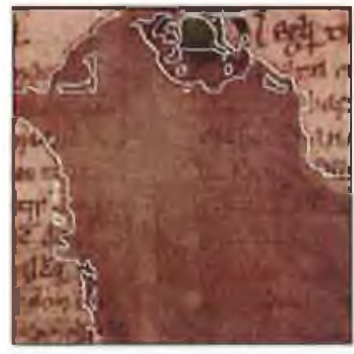

(1b)

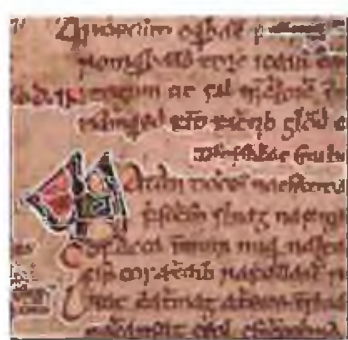

(3b)

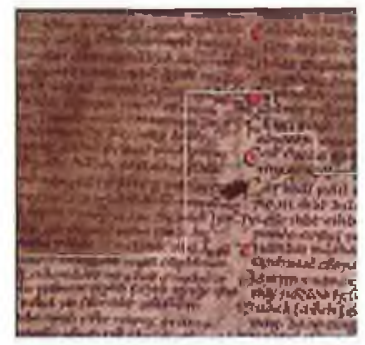

(2a)

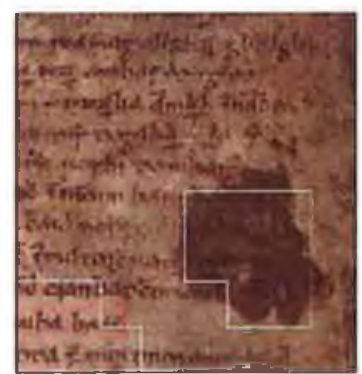

$(4 a)$

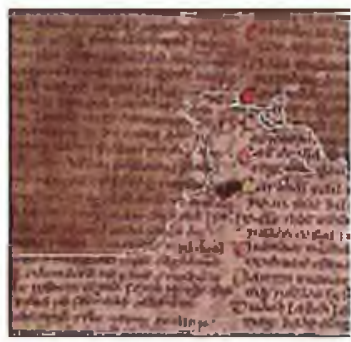

$(2 b)$

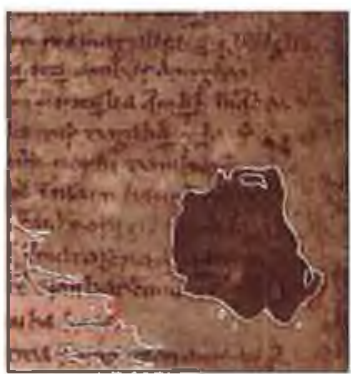

$(4 \mathrm{~b})$

Figure 6.1: (1a), (2a), (3a) and (4a) represents segmented results of ISOS images using Method-III, (1b), (2b), (3b), (4b) represents results of ISOS images using Method-III after the boundary refinement stage

The ISOS images are available in joint photographic experts group (jpeg) format. In this study, the jpeg images were converted into bitmap format for colour texture segmentation. The ability to segment a document into functionally different parts has been an ongoing goal of segmentation of the document analysis research. Segmentation of the old document images helps to determine the amount of damage in the document, caused by the afore-mentioned factors. This encompasses decomposing a document into its various corrupted components. This provides information on the amount of care to be taken to preserve the document from further damage. Four ISOS images were considered as the application database.

Figure 6.1-1a, 1b illustrates the correct identification of the stained regions using 
Method-III on the script images. A small portion of the green region was located properly. Figure 6.1-2a, $2 \mathrm{~b}$ shows the tarnished region. Figure 6.1-3a, 3b demonstrates the segmentation of the soiled region and the unsoiled region. Also one large coloured script was identified precisely. Figure 6.1-4a, 4b categorises the discoloured and the blemish regions. Colour plays a vital role in the developed framework which is evident from the presented results. Though the small scripts were not segmented separately, the damaged region and the different colours in the script were identified properly. The quantification of the segmentation of the ISOS images was based on the ground truth images. A boundary was drawn around the stained regions in the image and this regions were considered as the ground truth for quantification. The average segmentation error for four script images was found to be $2.6 \%$.

\subsubsection{Skin Cancer Images}

The skin cancer images were taken from the website [95]. Skin cancer is the most prevalent form of human cancer. Over exposure to sun is the cause of skin cancer. Skin cancer is a malignant tumour of the skin. There are different types of skin cancer and some are likely to be fatal. Skin cancers can be classified into melanoma and non-melanoma. Melanoma is the most dangerous form of skin cancer. It can spread through the whole body and is usually fatal if it does. If detected early, the cure rate for melanoma is almost 100 percent. Late detection, when the melanoma is more than three millimeters deep, results in only a 59 percent survival rate. Melanomas are much less common than non-melanomas, but they account for most of the mortality from skin cancers. Detection of malignant melanoma in its early stages considerably reduces morbidity and mortality [96]. Early detection also saves hundreds of millions of euros that is spent on the advanced disease. People are considered more at risk if they have lots of moles, are fair skinned with blue eyes, tend to sunburn easily or have freckles [97]. The rate 
of melanoma cases worldwide is increasing faster than any other cancer, with an annual rate of increase of six percent. Since 1973, the mortality from melanoma has increased by 50 percent.

Clinical features of pigmented lesions suggestive of skin cancer are known as the ABCD's of the skin cancer:

- asymmetry

- border irregularity

- colour variation

- diameter greater than $6 \mathrm{~mm}$.

There are various image analysis techniques developed to measure these features. Measurement of image features for diagnosis of the skin cancer images requires the detection of the lesions and localisation in an image. It is essential to determine the lesion boundaries accurately so that the measurements such as maximum diameter, irregularity of the boundary, and colour characteristics can be accurately computed. As a first step in skin cancer identification, the lesion boundaries are delineated by various image segmentation techniques. In this research work, colour and texture information from an image is used for the segmentation of the lesion boundaries. The segmentation helps to diagnose the skin lesions in the early stages. The skin cancer images obtained from the references [95] are in graphics interchange format (gif). They were converted to bitmap format to apply the segmentation process. Five skin cancer images were considered for the application database.

The skin lesions have complex structure, large variations in size as well as complex colours in the skin. The lesion is in contrast to the surrounding skin. The borders of lesions are not always well defined which makes the segmentation more 
CHAPTER 6. APPLICATION OF THE DEVELOPED COLOUR TEXTURE SEGMENTATION METHODOLOGY

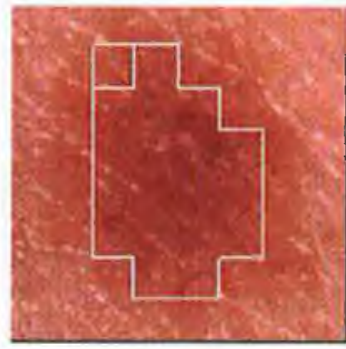

(1a)

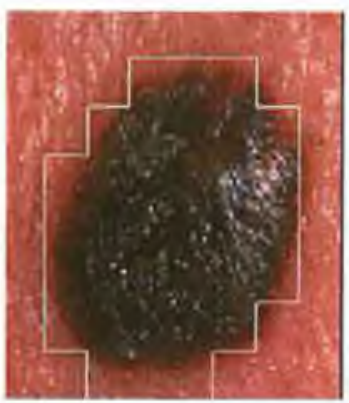

(3a)

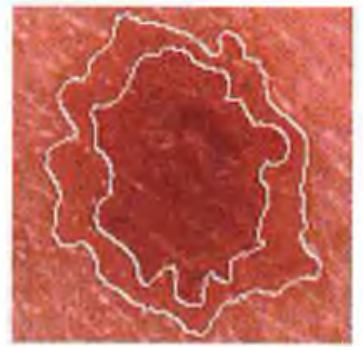

(1b)

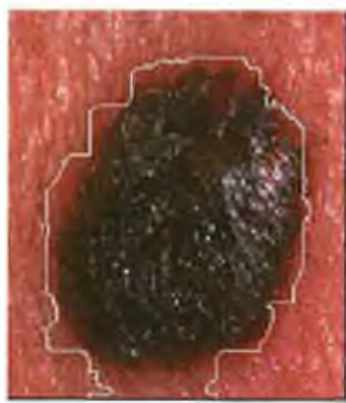

(3b)

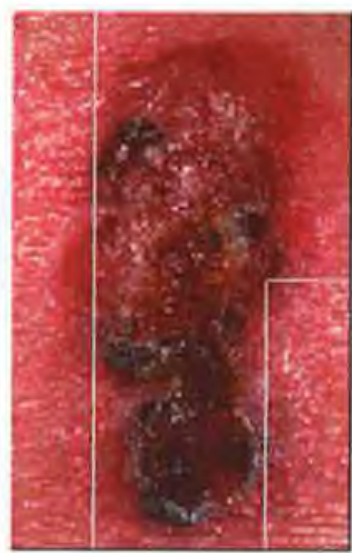

(5a)

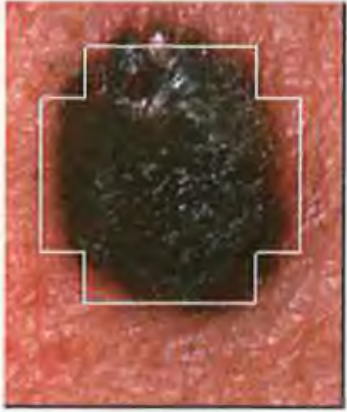

$(2 a)$

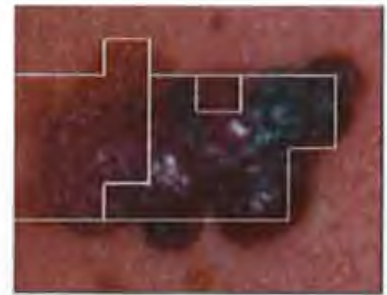

$(4 a)$

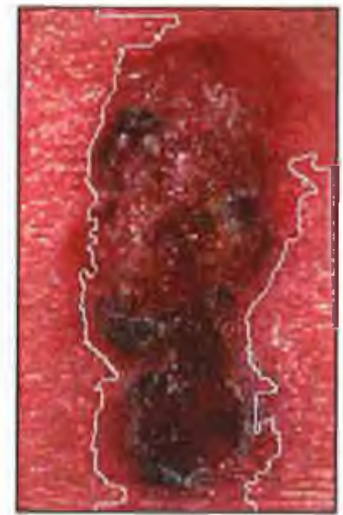

$(5 b)$

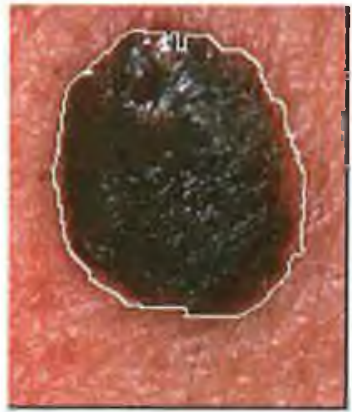

$(2 b)$

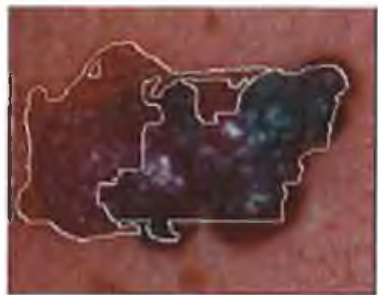

$(4 b)$

Figure 6.2: (1a), (2a), (3a), (4a), (5a) represents segmented results of skin cancer images from Method-III, (1b), (2b), (3b), (4b), (5b) represents the results after the boundary refinement stage 
complex. To analyse skin lesions, it is necessary to accurately locate and isolate the lesions. The efficient performance of the proposed colour texture segmentation method recognised the boundaries in the skin lesions exactly as shown in Figure 6.2.

Colour is one of the significant features in the examination of a skin lesion. Typical examples of lesions show reddish, bluish, grey and black areas and spots. Figure 6.2-1a, 1b, shows the segmentation of the skin lesion. The fine variation in the colour is identified and segmented accurately. Figure 6.2-2a, 2b, Figure 6.23a, 3b, Figure 6.2-4a, 4b and Figure 6.2-5a, 5b, illustrate the segmented results of the skin lesion. This segmentation clearly identifies the difference in colours in the skin lesion. The distribution of texture and colour features presents significant information, hence the segmentation based on the two features seems to be appropriate. This allows for the isolation of the lesion from healthy skin and extracts homogeneous coloured regions separately. The quantification of the skin lesion segmentation was based on visual results. The experimental results obtained proved to be encouraging and indicate that this method of colour texture segmentation is appropriate to be applied for detection of skin cancer images.

\subsubsection{Sediment Profile Imagery}

Sediment Profile Imagery (SPI) is a remote sensing technique that is used to determine whether the marine sediments provide suitable habitat for bottom dwelling fauna. This is an innovative and cost efficient method of surveying and monitoring lake or marine aquatic environments. The traditional method of sample collection and subsequent laboratory analysis is time consuming and expensive and data return time is slow. SPI is based on single lens reflex (SLR) camera photography and computer-based image analysis which greatly accelerates the time required to write reports and provide relevant data. The physical, 
chemical and biological features associated with organic enrichment are imaged and measured with the SPI system. The segmentation of the SPI images is the preliminary step in most pictorial pattern recognition and scene analysis problems. The underwater images are difficult to handle since they are acquired by

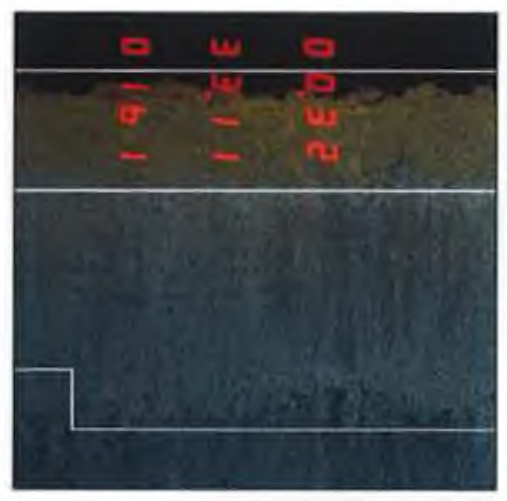

(1a)

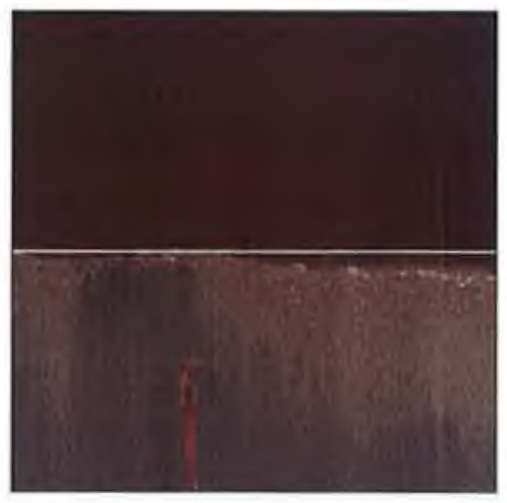

(2a)

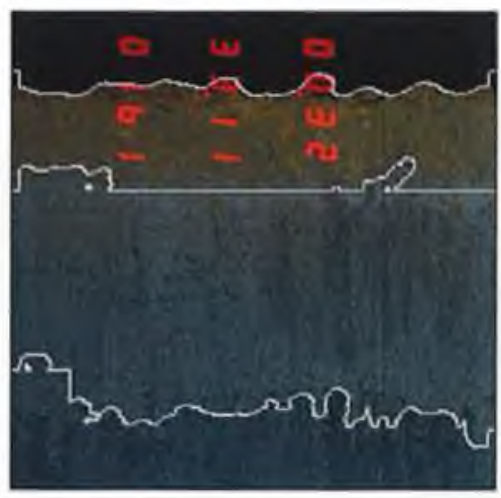

(1b)

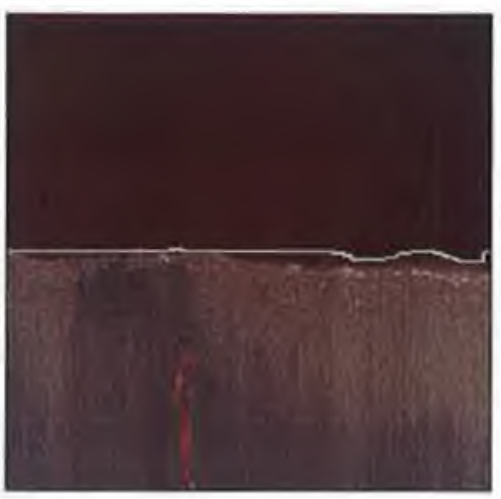

$(2 \mathrm{~b})$

Figure 6.3: (1a), (2a) represents segmented results of SPI images using MethodIII, (1b), (2b) represents segmentation results after the boundary refinement stage

a camera looking at the sea floor. These images are hard to process due to the light absorption, changing image radiance and lack of well defined features. The underwater images show fluctuating oxygenation levels under different organic loading and hydrographic conditions. Figure 6.3, shows the results after initial 
segmentation and after boundary refinement stages.

Figure 6.3-1a, 1b shows an example of a sediment image under high organic loading stress in hypoxic conditions. This is an example of a heavily impacted sediment. There is a clear difference in colour between the sediment surface layer and that lying under it. The colour texture segmentation clearly identifies different layers. Figure 6.3-2a, $2 \mathrm{~b}$ represents the sediment image with burrowing marine worms. The opportunistic worms thrive in high organic loading conditions and their burrowing action can often reintroduce oxygen into depleted sediments. Due to the thin feature difference in the organic sediment and the worm, the colour texture distribution could not identify the worm separately. But the sediments were segmented accurately. The quantification of the application was based on the segmentation of the oxidised layers by Ovidiu et al. [98]. The segmented result indicates that the developed framework for colour texture segmentation is able to identify the different sediments in the image.

The goal for the application is to find the performance of the colour texture segmentation method (Method-III which is selected as the best performer) in the script images, skin cancer images and in the detection of underwater images. All these applications use different images taken from different cameras and varying environment. In spite of these differences, the proposed colour texture segmentation method was able to identify different colour textured regions in an image and the resulting segmentation was appropriate and visually acceptable. In all the three applications, colour is a determinant factor in the segmentation process. The role of colour information in the colour texture segmentation is very significant. 
CHAPTER 6. APPLICATION OF THE DEVELOPED COLOUR TEXTURE SEGMENTATION METHODOLOGY

\subsection{Summary}

The proposed algorithm, Method-III was tested in three different applications, ISOS script images, skin cancer images and the underwater images. The algorithm was applied on the images and was found to produce proper segmentation results. 


\section{Chapter 7}

\section{Conclusions}

The primary objective of this thesis is to investigate the use of colour features for colour texture segmentation and to construct a framework for colour texture segmentation. Some methods are available for colour texture segmentation, but none of them is accepted as a standard model for colour texture segmentation. Absence of a universally accepted model for colour texture segmentation and the existence of a number of applications for colour texture segmentation formed the motivating factor for this research work. In this research work, a framework was developed for colour texture segmentation using colour and texture features. Three methods were developed in succession for colour texture segmentation. In addition, different texture techniques such as LBP, DCT were compared. The developed framework was implemented in different colour spaces such as RGB, YIQ and HSI. The three different methods of implementing the framework were also compared. This study was able to identify the drawbacks of the initially developed two methods and an attempt has been made to generate a methodology which is more effective and efficient. Three applications were experimented using the best methodology. Detailed results on the methodology have been presented in the previous chapters.

This chapter presents the importance of this research work, and how well this 
study addresses the issues related to colour texture segmentation. A few of the key results are quoted from the previous chapters. Useful conclusions on the performance of the three methods are also presented.

Segmentation of images is important and useful for many applications from an image processing perspective. Natural images consist of different objects with varying colours and textures. The segmentation of natural images is a complex problem due to the irregular boundaries of the objects in natural scenes. Colour and texture features play a vital role in the segmentation of images. The texture and colour are intrinsic features of an image and are essential for segmentation. The addition of colour to texture adds more information to segmentation.

The first step in colour texture segmentation is the feature extraction for colour textures. Separate processing of texture and colour and unification of colour and texture features for colour texture description is followed in this research work. The second step is the selection of feature extraction techniques to build a framework for colour texture segmentation. The selection of simple but efficient feature extraction techniques that are widely used is based on the literature review. The choice of LBP is due to its simplicity and efficiency. It is easy to implement LBP and it is invariant to any monotonic greyscale transformation. DCT is another texture feature extraction technique used for segmentation. Statistical features were extracted from colour planes using easy and straightforward techniques. An unsupervised colour clustering was used to cluster the input image by organising the data into $k$ different clusters. Adaptive smoothing smooths the image, removes the noise and restricts over segmentation. An unsupervised texture segmentation was adopted for the segmentation of colour texture images.

In Method-I, the grey scale techniques such as LBP and the filter based DCT approach were extended to colour images and the segmented results were ob- 
tained in each colour plane separately. Preliminary study was carried out on this single band method using LBP in various colour planes. The segmented results from different colour planes using Method-I varied according to the colour components present in the plane. It is not possible to draw a ground truth segmentation for each plane. In addition, no conclusion can be drawn on the colour planes based on the results from the individual planes. None of the colour planes can be ranked superior. The grey scale results illustrated better performance of $\mathrm{LBP} / \mathrm{C}$ than DCT. A quantification of the segmentation result is not possible using this method. The colour features were not explicitly used in this method. The above mentioned limitations in the single band method led to the development of Method-II.

In Method-II, texture and colour features were extracted from the luminance and the chrominance planes and processed in a combined way for colour texture segmentation. Method-II combines LBP/C or DCT based filter approach with statistical colour features. The distributions of colour and texture features were used for the colour texture description. The performance of different statistical features such as mean, standard deviation, energy and entropy was demonstrated. The performance shows that the colour feature entropy was consistent with (90.6\%) for LBP and (83.1\%) for DCT. No conclusion can be drawn based on the efficiency and effectiveness of the colour features. Also the best performing colour space among the three colour spaces was inconclusive. This is in agreement with the literature survey. The combination of LBP/C with statistical colour features performed better than the combination of DCT with statistical colour features in Method-II (indicated in Figure 5.15 in chapter 5). The influence of colour and texture are explicitly seen from the segmented images. This method has certain limitations such as poor boundary refinement using the pixelwise classification method. The results in the boundaries were not satisfactory and hence the development of Method-III was carried out. 
Method-III unified LBP/C or DCT based filter approach with unsupervised colour clustering. This method combines the distribution of texture and colour features to derive a similarity measure for colour texture description. This method of describing textures proved an efficient way to describe colour textures. The unsupervised texture segmentation developed by Ojala et al.[21] was modified and used for the colour texture segmentation. In Method-III, LBP/C with colour clustering performed better than DCT with colour clustering. The inclusion of a pre-processing technique, adaptive smoothing, avoids over segmentation and preserves the features. This is useful for the segmentation of natural images to define the exact boundaries. The weights for colour and texture together with the similarity measure Modified Kolmogorov Smirnov, increases the accuracy in discriminating various colour textured regions. This discriminates better than the G-statistic used in the earlier two methods. A boundary refinement method based on colour-clustered labels provides the enhancement of the boundaries. Similar to the previous methods, all the colour spaces performed equally well and none of the colour spaces proved to be superior. The quantisation of the colour and texture features were also presented in chapter 5. It was shown that the inclusion of the colour increases the segmentation performance. The results presented imply that the segmentation error is minimum for the colour weight 0.6 and texture weight 0.4 . The automatic inclusion of colour weight and texture weight as presented in chapter 5 produces an error rate of less than $1.0 \%$. The proposed framework for colour texture segmentation selects the weights automatically. Besides this, the visual examination illustrates the influence of the colour and texture.

The developed methodology for colour texture segmentation using LBP/C or DCT with colour clustering was applied to the Irish manuscript images, skin cancer images and the underwater images. The results were visually satisfactory and were quantified. The developed framework can be used in any colour texture 
image. From the results, it is clear that the same framework can be applied to any colour texture image without any modification of the parameters.

Based on the experimental results presented in this thesis, the following conclusions were drawn:

- From the overall performance of the three methods, Method-III produced good results and out performed other methods. This method based on the colour clustering and LBP/C or DCT distributions is suitable for the colour texture segmentation. Errors after agglomerative merging stage were recovered in Method-III using the boundary refinement algorithm. Method-III forms an excellent framework for colour texture segmentation.

- The colour texture analysis performed shows that colour alone or texture alone cannot provide a proper segmentation. The proper way of inclusion of colour and texture resulted in a better colour texture segmentation.

- The experiments outlined in this thesis illustrate that the use of colour improves the performance of texture analysis. The inclusion of colour in an appropriate way provides an efficient and effective colour texture segmentation. 


\section{Chapter 8}

\section{Thesis Contribution and Future Work}

\subsection{Thesis Contribution}

Based on the details reported in this thesis the author believes that the following new contributions have been made by this work. The contribution of this thesis can be divided into two categories. They are major ideas and contributions and minor ideas and contributions.

\section{Major ideas and contributions}

A novel framework based on the combination of colour and texture features was developed for colour texture segmentation. This methodology constructs the segmentation method utilising various techniques from image processing analysis. The $\mathrm{LBP} / \mathrm{C}$ or DCT texture features were extracted from the luminance plane and an unsupervised $k$-means colour clustering technique is used to cluster pixels in the chrominance planes. The distribution of colour features and the distribution of texture features are used to derive the similarity measure. This forms an effective way of discriminating colour textures. A pre-processing 
method, adaptive smoothing, is used to smooth the image before it is clustered. The use of adaptive smoothing for segmentation plays an imperative role for the preservation of features and the removal of noise in the image. A novel non-parametric test Modified-Kolmogorov Smirnov statistic is introduced in this framework as a similarity measure to discriminate the distributions of colour and texture features. The M-KS as developed in this work is a normalised statistic that considers the sum of the absolute value of the discrepancies between the normalised cumulative distributions compared to the KS statistic which considers the maximum of the absolute value of the discrepancies between the cumulative distributions.

In addition, an unsupervised segmentation was followed for splitting and merging the image. This uses a simple merging condition. Prior knowledge about the number of types of textures is not required in the unsupervised segmentation method followed. The modified version of the unsupervised segmentation described by Ojala [21] using texture and colour, is also considered as a thesis contribution. The method is applied for greyscale images in [21]. In this research work the method is extended with additional techniques for processing colour features such as adaptive smoothing and k-means clustering. Inclusion of colour information in the existing framework for greyscale improves the developed segmentation framework. The creation of a new boundary refinement technique adds to the contribution. The boundary refinement technique considers the distribution of the colour clustering for discriminating the pixels in the boundary. The amalgamation of colour and texture features to derive the similarity measure, the use of adaptive smoothing, the Modified-Kolmogorov Smirnov statistic and the creation of boundary refinement method resulted in the development of a novel framework for colour texture segmentation. The use of adaptive smoothing, Modified Kolmogorov Smirnov to discriminate the colour textured regions, the boundary refinement based on colour clustered labels and 
the definition of colour and texture weights for colour texture description forms the novelty in the proposed colour texture segmentation method. The fusion of the cues enhances the reliability of the segmentation. From the segmentation results it is evident that the developed method is effective and efficient. Three different applications from three different fields were chosen for colour texture segmentation. The use of the developed colour texture segmentation framework in particular applications such as ISOS images, skin cancer images and the SPI images adds to the contributions. The developed framework can be used in any colour texture image. From the results, it is clear that the same framework can be applied without any modification of the parameters. This research work emphasises the fact that the colour feature is an important contribution for colour texture segmentation.

\section{Minor ideas and contributions}

The minor contributions of this research work includes the use of standard statistical features for colour feature extraction in Method-II. Method-II is a straightforward, simple framework that combines colour and texture features for colour texture segmentation. Though Method-II was not dominant among the three methods, this method illustrates a new way of combining colour and textures for colour texture processing. Different colour features are extracted from the colour planes and combined for colour texture description. There is not much difference between the results obtained by using the same colour feature from the two planes and the results obtained by using different colour features from the two planes. A novel data structure was proposed for the implementation of unsupervised texture segmentation. The developed data structure used a quadtree which splits the image and a mergegraph to merge the adjoining regions. In addition to this, the implementation of the framework and the $C++$ program developed for the colour texture segmentation also contributes to the thesis. 


\subsection{Future Work}

Several improvements of the developed framework for colour texture segmentation are possible and some of them are listed below. Further research can be directed to the following topics:

1. The LBP texture feature extraction technique in the developed framework can be replaced by the extensions of the grey scale invariant texture analysis technique LBP. The extensions of the LBP includes the analysis of textures with multiple scales by combining neighbourhoods with different sizes [22].

2. The texture feature extraction techniques can be substituted by an equivalent or more efficient technique.

3. The proposed method is not ensured for invariance to variations in rotation, translation and scale. The proposed method can be upgraded to be invariant to variations in rotation, translation and scale.

4. The LBP can be expanded for translation and scale invariance. A rotation invariant LBP was developed by Ojala et al. [43]. But the LBP was not developed for invariance to translation and scale. LBP can be developed for translation and scale invariance.

5. The framework has split threshold, merge threshold and minimum block size values to be supplied for the colour texture segmentation. The future framework for colour texture segmentation may be incorporated with automatic selection of the threshold parameters and the minimum block size.

6. Apart from the features used in this study, the framework can be expanded for new colour and texture features. 
7. The proposed technique can be extended to handle complex images and other applications such as segmentation or ground classification of aerial images and medical images.

8. Colour clustered labels are used for the boundary refinement in this study. The addition of texture information with colour clustered labels may improve the segmentation results at the boundaries between regions with similar colour and different textures. 


\section{Bibliography}

[1] M.Petrou, M.Mirmehdi and M.Coors. "Perceptual Smoothing and Segmentation of Colour Textures". 5th European Conference on Computer Vision, Germany, 1:623-639, 1998.

[2] M.Tuceryan and A.K.Jain. "Texture Analysis". The Handbook of Pattern Recognition and Computer Vision, Second Edition, by C.H.Chen, L.F.Pau, P.S.P.Wang (editors), 207-248, World Scientific Publishing Co., 1998.

[3] M.Pietikainen and T.Ojala. "Texture Analysis in Industrial Applications". Advances in Image Processing and Machine Vision, Springer-Verlag, 1994.

[4] J.Sklansky, "Image segmentation and feature extraction". IEEE Trans on Sys, Man and Cyb, SMC-8, 237-247, 1978.

[5] R.G.Wilson and M.Spann, "Image Segmentation and the problem of uncertainity". Research studies Press (Ed.J.Kittler), 1987.

[6] A.K.Jain, "Fundamentals of Digital Image Processing". Prentice-Hall of India, 1989.

[7] IEEE Standard 610.4-1990, "IEEE Standard Glossary of Image Processing and Pattern Recognition Terminology". IEEE Press, New York, 1990.

[8] R.C.Gonzalez and R.E.Woods, "Digital Image Processing". AddisonWesley Publishing Company, 1992. 
[9] R.M.Haralick and L.G.Shapiro. "Computer and Robot Vision". Vol.1, Addison-Wesley Publishing Company, 1992.

[10] Bernd Jahne, "Digital Image Processing". Springer-Verlag, 1995.

[11] R.M.Haralick. "Statistical and structural approaches to texture". Proc. of IEEE, 67(5):786-803, 1979.

[12] Jussi Parkinen. "Spectral Colour Imaging". Invited Talk, 13th Scandinavian Conf. on Image Analysis, Sweden, 2003.

[13] Symon D'O. Cotton, "Colour, colour spaces and the human visual system, 1995.

[14] A.Drimbarean and P.F.Whelan. "Experiments in colour texture analysis". Pattern Recognition Letters, 22:1161-1167, 2001.

[15] Synthesis Image. http://www.cs.utah.edu/ michael/ts/examples.html.

[16] William K.Pratt. "Digital image processing". Second Edition, John Wiley and Sons, Inc., 1991.

[17] T.Ojala and M.Pietikainen. "Texture Classification, University of Oulu". http://www.ee.oulu.fi/research/imag/texture.

[18] S.J.Sangwine. "Colour in image processing". Electonics and communication Engg journal, 2000.

[19] M.Singh, M.Markou and S.Singh. "Colour image texture analysis: Dependence on colour space". Proc. 15th International Conference on Pattern Recognition, ICPR'02, Canada, 11-15, 2002.

[20] Q.T.Luong, “ Color in Computer Vision. The Handbook of Pattern Recognition and Computer Vision, Second Edition, by C.H.Chen, L.F.Pau, P.S.P.Wang (editors), pp.311-368, World Scientific Publishing Co., 1998. 
[21] T. Ojala and M.Pietikainen . "Unsupervised texture segmentation using feature distributions". Pattern Recognition, 32:477-486, 1999.

[22] T.Maenpaa. "The local binary pattern approach to texture analysis - Extensions and applications - Ph.D Thesis". University of Oulu, 2003.

[23] T.Caelli and D.Reye. "On the classification of image regions by colour, texture and shape". Pattern Recognition, 26(4):461-470, 1993.

[24] D.K.Panjwani and G.Healey. "Markov Random Field models for unsupervised segmentation of textured colour images". IEEE Transactions on Pattern analysis and Machine Intelligence, 17(10):939-954, 1995.

[25] A.K.Jain and G.Healey. "A multi-scale representation including opponent colour features for texture recognition". IEEE Trans on Image Processing, $7(1): 124-128,1998$.

[26] T.Maenpaa, M.Pietikainen and J.Viertola. "Separating colour and pattern information for colour texture discrimination". Proc. 15th International Conference on Pattern Recognition, ICPR'02, Canada, 668-671, 2002.

[27] VisTex. Colour texture image database. http://www-white.media.mit.edu/vismod/imagery/VisionTexture/vistex.html, 2000 .

[28] M.Unser and M.Eden. "Multiresolution feature extraction and selection for texture segmentation". IEEE Trans on PAMI, 11(7):717-725, 1989.

[29] A.K.Jain and F.Farrokhnia. "Unsupervised texture segmentation using Gabor filters". Pattern Recognition, 24(12):1167-1186, 1991.

[30] D.Dunn and W.E.Higgins. "Optimal Gabor filters for texture segmentation". IEEE Trans. on Image Processing, 4(7):947-964, 1995. 
[31] J.Puzicha, T.Hofmann and J.M.Buhmann. "Non-parametric similarity measures for unsupervised texture segmenattion and image retrieval". Proc. of IEEE Conf. on Computer Vision and Pattern Recognition, San Juan, 1997.

[32] T.Hofmann, J.Puzicha and J.M.Buhmann. "Unsupervised texture segmentation in a deterministic annealing framework". IEEE Transactions on Pattern Analysis and Machine Intelligence, 20(8): 803-818, 1998.

[33] T.Randen and J.H.Husoy. "Texture segmentation using filters with optimised energy separation". IEEE Trans. on Image Processing, 8(4):571-582, 1999.

[34] Lei Wang and Jun Liu. "Texture segmentation based on MRMRF modeling". Pattern Recognition Letters 21, 189-200, 2000.

[35] L.Wang and D.He. "Texture classification using texture spectrum". Pattern Recognition, 23(8):905-910, 1990.

[36] P.Brodatz. Texture-A photographic Album for Artists and Designers. Reinfold, NewYork, 1968.

[37] Michael Unser. "Texture classification and segmentation using wavelet frames". IEEE Trans. on Image Processing, 4(11):1549-1560, 1995.

[38] D.Harwood, T.Ojala, M.Pietikainen, S.Kelman and L.Davis. "Texture classification by center-symmetric auto-correlation, using Kullback discrimination of distributions". Pattern Recognition Letters, 16:1-10, 1995.

[39] T.Ojala, M.Pietikainen and D.Harwood. "A comparative study of texture measures with classification based on feature distributions". Pattern Recognition, 29(1):51-59, 1996. 
[40] T.Randen and J.H.Husoy. "Filtering for texture classification: A comparative study". IEEE Trans on Pattern Analysis and Machine Intelligence, 291-310, 1999.

[41] Lei Wang and Jun Liu. "Texture classification using multi-resolution Markov random field models". Pattern Recognition Letters 20, 171-182, 1999.

[42] T.Maenpaa, M.Pietikainen and T.Ojala. "Texture classification by multipredicate local binary pattern operators". 15th Int. conf. on Pattern Recognition, Spain, 3:951-954, 2000.

[43] T.Ojala, M.Pietikainen and T.Maenpaa. "Gray scale and rotation invariant texture classification with local binary patterns". Proc. Sixth European Conference on Computer Vision, June 26 - July 1, Dublin, Ireland, 1:404-420, 2000.

[44] M.Pietikainen, T.Ojala and Z.Xu. "Rotation-invariant texture classification using feature distributions". Pattern Recognition, 33:43-52, 2000.

[45] F.Lahajnar and S.Kovacic. "Rotation-invariant texture classification". Pattern Recognition Letters 24, 1151-1161, 2003.

[46] T.Maenpaa and M.Pietikainen. "Multi-scale binary patterns for texture analysis". 13th Scandinavian Conf. on Image Analysis, Sweden, 885-892, 2003.

[47] Outex. Texture image database.

http://www.outex.oulu.f/outex.php, 2001.

[48] M.Turtinen, T.Maenpaa and M.Pietikainen. "Texture classification by combining local binary pattern features and self-organising maps". 13th Scandinavian Conf. on Image Analysis, Sweden, 1162-1170, 2003. 
[49] M.Unser. "Local linear transforms for textrue measurements". Signal Processing, 11:61-79, 1986 .

[50] Lee Hok Siew, Robert M.Hodgson and Errol J.Wood. "Texture measures for carpet wear assessment". IEEE Trans. on PAMI, 10:92-105, 1988 .

[51] P.P.Ohanian and R.C.Dubes. "Performance evaluation for four classes of textural features". Pattern Recognition, 25(8):819-833, 1992.

[52] I.Ng, T.Tan and J.Kittler. "On local linear transform and gabor filter representation of texture". Proc.Int.Conf.Pattern Recognition, 627-631, 1992.

[53] B.S.Manjunath and W.Y.Ma. "Texture features for browsing and retrieval of image data". IEEE Trans. on Pattern Analysis and Machine Intelligence, 18(8):1996.

[54] Yong Rui, Thomas S.Huang and Shih-Fu Chang. "Image retrieval: Past, present and future". Proc. of international symposium on multimedia information processing, 1997.

[55] Chen-Chang Chen and Chaur-Chin Chen. "Filtering methods for texture discrimination". Pattern Recognition Letters 20, 783-790, 1999.

[56] M.Pietikainen and T.Ojala. "Nonparametric texture analysis with simple spatial operators". Proc. QCAV'99 5th International Conference on Quality Control by Artificial Vision, 11-16, 1999.

[57] T.Ojala, K.Valkealahti, E.Oja and M.Pietikainen. "Texture discrimination with multidimensional distributions of signed differences". Proc. Fourth Asian Conference on Computer Vision, Taiwan, 2:1082-1088, 2000.

[58] S.Singh and M.Sharma "Texture analysis experiments with Meastex and Vistex benchmarks". ICAPR, 417-424, 2001. 
[59] Meas'Tex. Texture Image Database.

http://www.cssip.uq.edu.au/staff/meastex/www/obtaining.html, 2000.

[60] M.Singh and S.Singh. "Spatial texture analysis: A comparative study". Proc. 15th International Conference on Pattern Recognition ICPR'02, Quebec, 11-15 August, 2002.

[61] J.Zhang and T.Tan. "Brief review of invariant texture analysis methods". Pattern Recognition, 35:735-747, 2002.

[62] W.Skarbek and A.Koschan. "Color Image Segmentation: A survey". Technical report, Technical University Berlin, 1994.

[63] L.Shafarenko, M.Petrou, and J. Kittler. "Histogram-based segmentation in a perceptually uniform color space". IEEE Transactions on Image Processing, $7(9): 1354-1358,1998$.

[64] L.Lucchese and S.K.Mitra. "Unsupervised low-frequency driven segmentation of color images". Proc. IEEE Int. Conf on Image Processing, 240-244, 1999.

[65] S.Wesolkowski and P.Fieguth. "Colour image segmentation using a region growing method". 9th congress of the Int. Colour Association, Rochester, NewYork, USA, 2001.

[66] Tie Qi Chen and Yi Lu. "Colour image segmentation-an innovative approach". Pattern Recognition, 35:395-405, 2002.

[67] H.D.Cheng, X.H.Jiang and J.Wang "Colour image segmentation based on homogram thresholding and region merging". Pattern Recognition, 35:373393, 2002. 
[68] M.P.D.Jolly and A.Gupta. "Colour and texture fusion: Application to aerial image segmentation and GIS updating". IEEE Workshop on Applications of Computer Vision, 2-7, 1996.

[69] L.Shafarenko, M.Petrou and J.Kittler. "Automatic watershed segmentation of randomly textured colour images". IEEE Trans. on Image Processing, 6(11):1530-1543, 1997.

[70] A.Tremeau and N.Borel. "A region growing and merging algorithm to colour segmentation". Pattern Recognition, 30(7):1191-1203, 1997.

[71] L.Lucchese and S.K.Mitra. "Unsupervised segmentation of colour images based on k-means clustering in the chromaticity plane". Proc. of IEEE workshop on content based access of image and video libraries, 74-78, 1999.

[72] G.Paschos and K.P.Valavanis. "A colour texture based visual monitoring system for automated surveillance". IEEE Trans. on systems, man and cybernetics-Part C: Applications and Reviews, 29(2):298-307, 1999.

[73] Kan-Min Chen and Shu-Yuan Chen. "Colour texture segmentation using feature distributions". Pattern Recognition Letters, 23:755-771, 2002.

[74] S.C.Tan and J.Kittler. "Colour texture classification using features from colour histogram". Proc. Eigth scandinavian conf. on Image Processing, 1993.

[75] G.Van de Wouwer, S.Livens, P.Scheunders and D.Van Dyck. "Colour texture classification by wavelet energy correlation signatures". Pattern Recognition, 32:443-451, 1999 .

[76] G.Paschos. "Fast colour recognition using chromaticity moments". Pattern Recognition Letters, 21:837-841, 2000. 
[77] M.Pietikainen, T.Maenpaa and J.Viertola. "Color texture classification with color histograms and local binary pattern". Proc. Texture2002. 2nd International Workshop on Texture Analysis and Synthesis. In Conjunction with ECCV2002, 2002.

[78] G.Paschos and M.Petrou. "Histogram ratio features for colour texture classification". Pattern Recognition, 2002.

[79] J.R.Smith and S.F.Chang. "Automated image retrieval using color and texture". Columbia University CTR, TechREPORT, 414-95-20, 1995.

[80] T.Randen, A.Jain and J.H.Husoy. "Image content search by colour and texture properties". Proc. IEEE Int. Conf. on Image Processing, California, 1997.

[81] M.S.Kankanhalli, B.M.Mehtre and H.Y.Huang. "Colour and spatial feature for content-based image retrieval". Pattern Recognition Letters, 20:109-118, 1999.

[82] Q.Iqbal and J.K.Aggarwal. "Combining structure, colour and texture for image retrieval: A performance Evaluation". Int. Conf. on Pattern Recognition, ICPR, Canada, 2002.

[83] Cheng-Hao Yao and Shu-Yuan Chen. "Retrieval of translated, rotated and scaled colour textures". Pattern Recognition Letters, 36:913-929, 2003.

[84] K.Y.Song, J.Kittler and M.Petrou. "Defect detection in random colour textures". Image and Vision Computing, 14:667-683, 1996.

[85] J.Kyllonen and M.Pietikainen. "Visual inspection of parquet slabs by combining colour and texture". IAPR workshop on machine vision applications, Japan, 2000. 
[86] M.Mirmehdi and M.Petrou. "Perceptual versus gaussian smoothing for pattern-colour separability". Int. Conf. on Signal Processing and Communications, 136-140, 1998.

[87] J.Puzicha, J.M.Buhmann, Y.Rubner and C.Tomasi. "Empirical evaluation of dissimilarity measures for colour and textrue". Proc. of IEEE Int. Conf. on Computer Vision, ICCV'99, 1156-1173, 1999.

[88] K.Messer and J.Kittler. "A region-based image database system using colour and texture". Pattern Recognition Letters, 1323-1330, 1999.

[89] K.R.Castleman, " Digital image processing, Second Edition, Prentice Hall, Inc. 1996.

[90] M.Petrou and P.Bosdogianni. "Image processing-The fundamentals", John Wiley and Sons, Ltd., 1999.

[91] R.O.Duda and P.E.Hart "Pattern classification and scene analysis". John Wiley and Sons, Inc., New York, 1973.

[92] A.K.Jain and R.C.Dubes. "Algorithms for clustering data". Prentice Hall, Advanced Reference Series, New Jersey, 1988.

[93] Ke Chen. "A feature preserving adaptive smoothing method for early vision". The Journal of Pattern Recognition Society, 33, 2000.

[94] M.Sonka, V.Hlavac and R.Boyle. "Image processing, analysis and machine vision". Second Edition, Chapman and Hall, 1995.

[95] Skin Cancer Images. "http://tray.dermatology.uiowa.edu/DermDB.htm". "http://dermatlas.med.jhmi.edu/derm/IndexDisplay.cfm?ImageID=1061935956". "http://matrix.ucdavis.edu/tumors/tradition/gallery-melanoma.html". 
[96] L.Xu, M.Jackowski, A.Ghoshtasby, D.Roseman, S.Bines, C.Yu, A.Dhawan and A.Huntley. "Segmentation of skin cancer images". Image and Vision Computing, 65-74, 1999.

[97] Skin Cancer Images. "http://www.cmis.csiro.au/iap/RecentProjects/melanoma.htm".

[98] Ovidiu Ghita, Paul.F.Whelan and R.Kennedy. "A practical approach for analysing SPI images". Systemics, Cybernetics and Informatics, 2003.

[99] P.F.Whelan and D.Molloy. "Machine vision algorithms in Java : Techniques and implementation". Springer-Verlag, London, 2000.

[100] B.Mandelbrot. "The fractal geometry of nature". Freeman, New York, 1983.

[101] P.Soille and J.Rivest. "On the validity of fractal dimension measurements in image processing". Journal of visual communication and image representation, 1996.

[102] A.Pentland. "Fractal-based description of natural textures". IEEE Trans. on PAMI, 6:356-369, 1984. 


\section{List of Publications}

N.Padmapriya, P.P.Pradeep and P.F.Whelan. (2002), A new data structure for the implementation of unsupervised texture segmentation, in 'Proceedings of the Irish Signals and Systems Conference (ISSC02)', pp.109-113, Cork, Ireland.

N.Padmapriya, O.Ghita and P.F.Whelan. (2003a), Experimentation on the use of Chromaticity Features, Local Binary Pattern and Discrete Cosine Transform in Colour Texture Analysis, in 'Proceedings of the 13th Scandinavian Conference on Image Analysis (SCIA03)', pp.186-192, Gotenburg, Sweden.

N.Padmapriya, O.Ghita and P.F.Whelan. (2003b), Investigation on the Discrete Cosine Transform and Chromaticity Features in Colour Texture Analysis, in 'Proceedings of the Irish Signals and Systems Conference (ISSC03)', pp.524528, Limerick, Ireland.

N.Padmapriya, O.Ghita and P.F.Whelan. (2004), Integration of Feature Distributions for Colour Texture Segmentation, 17th International Conference on Pattern Recognition (ICPR04)', Cambridge, United Kingdom. 


\section{Appendix A}

\section{Texture Feature Extraction}

\section{Techniques}

A number of techniques have been developed to describe and analyse different textures. The success in the field of texture analysis depends on the application. Most techniques are computationally intensive and considered impractical for any application. Hence, simple and easy to implement techniques such as LBP and filter based DCT approach are considered a better option for texture analysis. A brief overview of few texture analysis techniques are outlined below.

\section{A.1 Co-occurrence}

The spatial grey level dependence method or the co-occurrence method estimates second order statistics of the image and was suggested by Haralick [11]. This method of texture description is based on the repeated occurrence of some grey level configuration in the texture. This configuration varies rapidly with distance in fine textures and slowly in coarse textures. The $G \times G$ grey level co-occurrence matrix $P_{\phi, d}(i, j)$ is defined as the number of occurrences of the pair of grey levels $i$ and $j$ separated by a distance $d$, in the given direction $\phi$. The following example 
illustrates the co-occurrence matrix computation for the distance $d=1$ and $\phi=0$. A $4 \times 4$ image matrix with four grey levels is presented as follows:

$$
\left(\begin{array}{llll}
0 & 0 & 1 & 1 \\
0 & 0 & 1 & 1 \\
0 & 2 & 2 & 2 \\
2 & 2 & 3 & 3
\end{array}\right)
$$

subsequently, the matrix $P_{0^{\circ}, 1}$ is constructed as:

$$
P_{0^{\circ}, 1}=\left(\begin{array}{cccc}
4 & 2 & 1 & 0 \\
2 & 4 & 0 & 0 \\
1 & 0 & 6 & 1 \\
0 & 0 & 1 & 2
\end{array}\right)
$$

The element $P_{0^{\circ}, 1}(0,0)$ represents the number of times the two pixels with grey levels 0 and 0 appear, separated by a distance $d=1$ in the direction $0^{\circ}$. The value of $P_{0^{\circ}, 1}(0,0)=4$. The elements $P_{0^{\circ}, 1}(3,2)$ represents the number of times the two pixels with grey levels 3 and 2 appear, separated by a distance 1 in the direction $0^{\circ}, P_{0^{\circ}, 1}(3,2)=1$. Also $P_{0^{\circ}, 1}(2,3)=1$ due to the matrix symmetry. Similarly, the relative frequency matrix $P_{\phi, d}(i, j)$ is constructed for other directions and distances. Haralick extracted a set of 14 features from these matrices. The features are:

Energy:

$$
E=\sum_{i} \sum_{j}\left[P_{\phi, d}(i, j)\right]^{2}
$$

Energy is a measure of homogeneity of the image.

Entropy:

$$
E N T=-\sum_{i} \sum_{j} P_{\phi, d}(i, j) \log P_{\phi, d}(i, j)
$$


Entropy gives a measure of complexity of the image. Complex textures tend to have higher entropy.

Contrast:

$$
C O N=\sum_{i} \sum_{j}(i-j)^{2} P_{\phi, d}(i, j)
$$

Contrast is a measure of local variations present in an image.

Homogeneity:

$$
H=\sum_{i} \sum_{j} \frac{P_{\phi, d}(i, j)}{1-(i+j)^{2}}
$$

Homogeneity gives a degree to which similar grey levels tend to be neighbours. Correlation:

$$
C O R=\sum_{i} \sum_{j} \frac{\left(i-\mu_{x}\right)\left(j-\mu_{y}\right) * P_{\phi, d}(i, j)}{\sigma_{x} \sigma_{y}}
$$

where $\mu_{x}$ and $\sigma_{x}$ are the mean and standard deviation of the row sums of the matrix and $\mu_{y}$ and $\sigma_{y}$ are the statistics of the column sums. Correlation measures the grey level linear dependencies in an image.

Co-occurrence is extensively used and has become a benchmark in texture analysis. This method has been successfully applied in many image processing applications. The disadvantage of this method is its inability to describe the shape aspects of the texture [99].

\section{A.2 Gabor Filters}

The Gabor filtering approach has been widely used in texture analysis. The even symmetric Gabor filter approach adopted by Randen et al. [40] has the form:

$$
g(x, y)=\exp ^{-\frac{1}{2}\left(\frac{x^{2}}{\sigma_{x}^{2}}+\frac{y^{2}}{\sigma_{y}^{2}}\right)} \cos \left(2 \pi u_{0} x+\phi\right)
$$

where parameters $\left(\sigma_{x}, \sigma_{y}\right)$ characterise the spatial extent and the bandwidth of the filter, $u_{0}$ is the radial frequency and $\phi$ is the phase of the filter. To compute 
the texture features, the image is convolved with a bank of Gabor filters of different parameters. This is called multichannel filtering approach. The texture features are defined as the energy of the filtered images calculated as

$$
f_{m}=\frac{1}{M^{2}} \sum_{x=0}^{M} \sum_{y=0}^{M}\left|I * g_{m}\right|^{2}
$$

where $g_{m}$ is a Gabor filter defined by equation (A.6). The properties of Gabor filters make them suitable for texture analysis. They have tuneable parameters such as orientation and scale which can capture the underlying texture information. This approach is inspired by psychophysical research on the human visual system [14]. A complete review of the issues involved in Gabor filter design for texture segmentation can be found in [30].

\section{A.3 Markov Random Field}

Markov Random Field is popular for modelling images. They capture the local spatial texture information in an image. This model implies that the probability of a pixel taking a grey value is conditioned only on the grey levels of the pixels from its neighbourhood. The image is represented by an $N \times N$ lattice denoted by $S=\{(i, j) / 1 \leq i \leq N, 1 \leq j \leq N\}$. X is the random variable which represents the grey level value at pixel $(i, j)$ on the lattice $S$. Consider the neighbour set $N=\left\{N_{i}, i \varepsilon S\right\}$ where $N_{i}$ is the set of neighbouring points of $i$. The neighbourhood system $N_{i}$ must be symmetric. $i \varepsilon N_{j} \Longleftrightarrow j \varepsilon N_{i}$ and $i$ does not belong to $N_{i}$. The random field $\mathrm{X}$ is said to be an MRF on $S$ with respect to a neighbourhood system $N$ if and only if

$$
\begin{aligned}
& P(x)>0, \forall x \varepsilon X \\
& P\left(x_{i} / x_{S-i}\right)=P\left(x_{i} / x_{N_{i}}\right)
\end{aligned}
$$

The estimated parameters of the model were used to generate synthetic textures. MRF models have been applied to various image processing applications such as 
texture classification, texture segmentation and texture synthesis. The model can be fully described by a small and compact number of parameters. Its features are invariant to image rotation by $(k \pi) / 2$. The limitation of MRF is that they only account for the local interaction between the pixels and are sensitive to grey level distortions. The disadvantage of this technique is its computational complexity and it cannot model all the textures.

\section{A.4 Fractals}

Natural objects have a statistical quality of roughness and self-similarity at different scales. Fractals are very useful in modelling these properties. Natural objects such as clouds, mountain, water etc., can be accurately described and generated using fractal objects. This led to the use of fractal geometry in the analysis of textures, especially natural textures which exhibit a high degree of randomness. The parameter used in the fractal analysis of textures is a noninteger number called fractal dimension. Many algorithms have been developed to compute its value.

Mandelbrot [100] observed that the length $L_{\epsilon}$ of a curve $C$ would depend on the size $\epsilon$ of the measuring tool used. For a rectifiable curve (a curve with finite length) if $\epsilon$ tends to zero, the value of $L_{\varepsilon}$ represents the actual length of the curve $\mathrm{C}$. This does not happen with the fractal curves, the smaller $\epsilon$, the finest structure it passes over and $L_{\varepsilon}$ tends to infinity.

The fractal dimension $D$ of the curve $C$ is given by the equation (A.10)

$$
D(C)=\lim _{\epsilon \rightarrow 0}\left[1-\frac{\log \left(L_{\epsilon}\right)}{\log (\epsilon)}\right]
$$

For a fractal curve, $\mathrm{D}$ is a non-integer number larger than immediate geometrical dimension of the curve. The fractal dimension can be determined using geometrical and stochastic algorithms. A survey of these algorithms is given in [101]. 
One such method to calculate the fractal dimension is box-counting method. An arbitrary grid of boxes of side $s$ is first defined and the number of necessary boxes $N(s)$ to cover the curve is counted. This process is repeated with the grid of half size than the previous and it can continue infinitely. The fractal dimension is given by equation (A.11)

$$
D=1-\lim _{s \rightarrow 0} \frac{\log (N(s)}{\log (s)}
$$

A $\log N(s)$ against $\log (s)$ characteristic is plotted and the best fitted line between the points is estimated. Considering the slope of this line as $m$, the equation (A.11) becomes $D=1-m$.

There are a number of applications of fractal models in texture classification and segmentation [102], but the fractal geometry is mainly used in image compression and coding tasks. The fractal dimension is relatively insensitive to an image scaling and shows a strong correlation with the human judgement of surface roughness. Though textures were characterised by relatively small set of measures, the features require large computation [99]. 


\section{Appendix B}

\section{Colour Spaces}

A colour space is a means of uniquely specifying a colour. There are a number of colour spaces in common usage depending on a particular application involved. A detailed description of different colour spaces and their use in colour image analysis are found in [99]. A brief review of different colour space are outlined below.

\section{B.1 RGB Space}

RGB space is the simplest and most popular colour space, it is found in systems that use a cathode ray tube to display images. The RGB colour space is used in every computer system, scanners, image storage devices, television, video camera etc. The RGB colour space is made of three additive primaries: red, green and blue. RGB space is widely used for computer based applications and is often visualised by a unit cube, as in Figure B.1. Each colour (red, green, blue) is assigned to one of the three orthogonal coordinate axes in 3D space. RGB model is device dependent and excludes some visible colours. RGB space is perceptually non-uniform and consequently the distance will not represent the real difference between colours and a uniform quantisation gives unsatisfactory results [99]. In 


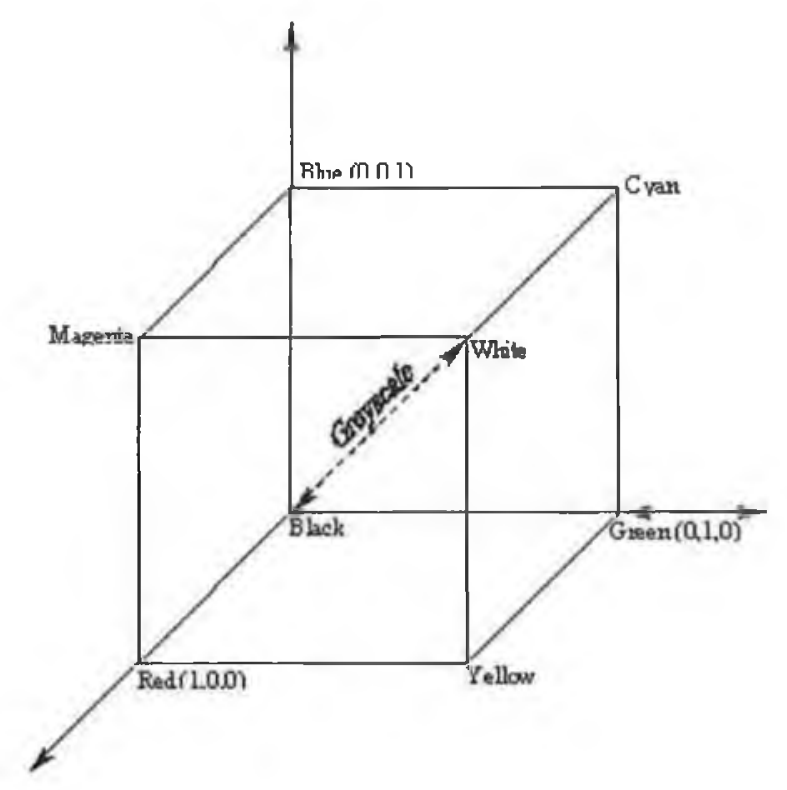

Figure B.1: RGB Colour Cube

order to make a better colour processing, the RGB space is converted into other colour spaces using a nonlinear transformation.

\section{B.2 HSI Space}

Many colour models are based on human visual perception, and one among the models is HSI colour space. HSI colour space represents colours in terms of Hue, Saturation and Intensity (HSI). This model is a two ended hexagonal pyramid as illustrated in Figure B.2. The middle hexagon has colours with three additive and three subtractive primaries. Black lies at a tip of the pyramid and white at the opposite tip of the pyramid. Thus the angle around the hexagon is the hue and the distance from the center axis is the saturation. The nonlinear relations used to convert RGB space to HSI space are :

$$
\begin{aligned}
& \text { Intensity } I=\frac{1}{3}(R+G+B) \\
& \text { Hue } H=\arccos \frac{2 R-G-B}{2 \sqrt{(R-G)+(R-B)(G-B)}}
\end{aligned}
$$




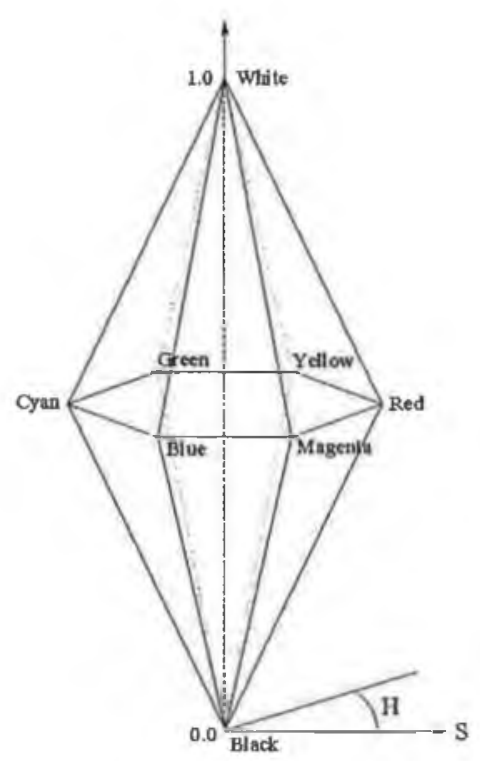

Figure B.2: Hexagonal Pyramid

$$
\text { Saturation } S=1-3 \frac{\min (R, G, B)}{R+G+B}
$$

where equation(B.1) corresponds to grey level of pixels in monochrome images. The advantage of the HSI colour space is that the grey scale algorithms can be applied to $\mathrm{I}$ component and $\mathrm{H}$ and $\mathrm{S}$ components can be used as a cue for image segmentation or for region growing algorithms. The focus in this research is on HSI colour space as the proposed method for colour texture segmentation uses the colour information from the chrominance planes and the grey level information from the luminance plane. The limitation of this space is the hue singularity. i.e., substituting $R=G=B=0$ in equation(B.2) hue $H$ is undefined. This is a very intuitive model for colour artists working with light.

\section{B.3 HSV Space}

Hue, Saturation and Value (HSV) space is similar to HSI space but with a single cone to describe the space. The application of HSV space is similar to that of 
HSI space. Black lies at the tip of the cone, white at the center of the base and hues around the perimeter of the base.

\section{B.4 YIQ Space}

YIQ space is the first of the colour spaces that attempts to emulate the human visual system. Transmitted colour signals formed by encoding RGB picture information is split into two parts, the luminance $\mathrm{Y}$ and the chrominance I and $\mathrm{Q}$. The colour hue is given by the chrominance angle $\mathrm{Q}$ and the saturation information is given by the chrominance amplitude I. Separating the intensity from other components has several advantages. This is because human eyes are more sensitive to the intensity than to the hue, the bits for encoding can be distributed more effectively. To get achromatic images, it is necessary to drop the chromatic part as used in NTSC (National Television System Committee). The YIQ has the advantage over other methods, in that the colour space separates as hue and intensity. Image processing is done on the intensity and colour plane separately. The YIQ space can be computed as a linear function of the RGB values. The transformation of the RGB signals are given by:

$$
\left(\begin{array}{l}
Y \\
I \\
Q
\end{array}\right)=\left(\begin{array}{ccc}
0.299 & 0.587 & 0.114 \\
0.596 & -0.275 & -0.321 \\
0.212 & -0.523 & 0.311
\end{array}\right)\left(\begin{array}{l}
R \\
G \\
B
\end{array}\right)
$$

Equation(B.4) is a simple representation of the NTSC encoding scheme used in American broadcast television. This is a device dependent colour space meaning that the actual colour depends on the type of monitor used. YIQ is a 3D cartesian coordinate system with the visible subset being a convex polyhedron that maps into the RGB cube. $\mathrm{Q}$ is orthogonal to I. More attention is paid to this space as this space is useful for the segmentation approach used in this research. According to Drimbarean and Whelan [14], high classification accuracy was obtained using this colour space. 


\section{B.5 YUV Space}

YUV is used in European television transmission systems. The transformation of RGB signals is as followed:

$$
\left(\begin{array}{l}
Y \\
U \\
V
\end{array}\right)=\left(\begin{array}{ccc}
0.299 & 0.587 & 0.114 \\
-0.147 & -0.289 & 0.437 \\
0.615 & -0.515 & -0.1
\end{array}\right)\left(\begin{array}{l}
R \\
G \\
B
\end{array}\right)
$$

The $\mathrm{I}$ and $\mathrm{Q}$ signals are related to $\mathrm{U}$ and $\mathrm{V}$ signals by a simple rotation of coordinates in colour space [16].

$$
I=-U \sin \left(33^{\circ}\right)+V \cos \left(33^{\circ}\right) \quad Q=U \cos \left(33^{\circ}\right)+V \sin \left(33^{\circ}\right)
$$

$\mathrm{YC}_{b} C_{r}, \mathrm{YCC}$ are the other colour spaces used in television transmission. These colour spaces are also known as transmission primaries. They separate luminance from chrominance and are useful in compression and image processing applications and are device dependent.

\section{B.6 CIE-XYZ Space}

International Commission on Illumination (CIE) has defined a system that classifies colour according to the human visual system. Using this system any colour can be specified in terms of CIE coordinates. CIE defined three standard primaries $\mathrm{X}, \mathrm{Y}$ and $\mathrm{Z}$ to replace red, green and blue since all visible colours could not be specified with the values of red, green and blue components. They can be calculated from R, G and B stimulus using the linear transformation

$$
\left(\begin{array}{l}
X \\
Y \\
Z
\end{array}\right)=\left(\begin{array}{ccc}
2.36 & -0.515 & 0.0052 \\
-0.89 & 1.42 & -0.014 \\
-0.46 & 0.88 & 1.009
\end{array}\right)\left(\begin{array}{l}
R \\
G \\
B
\end{array}\right)
$$




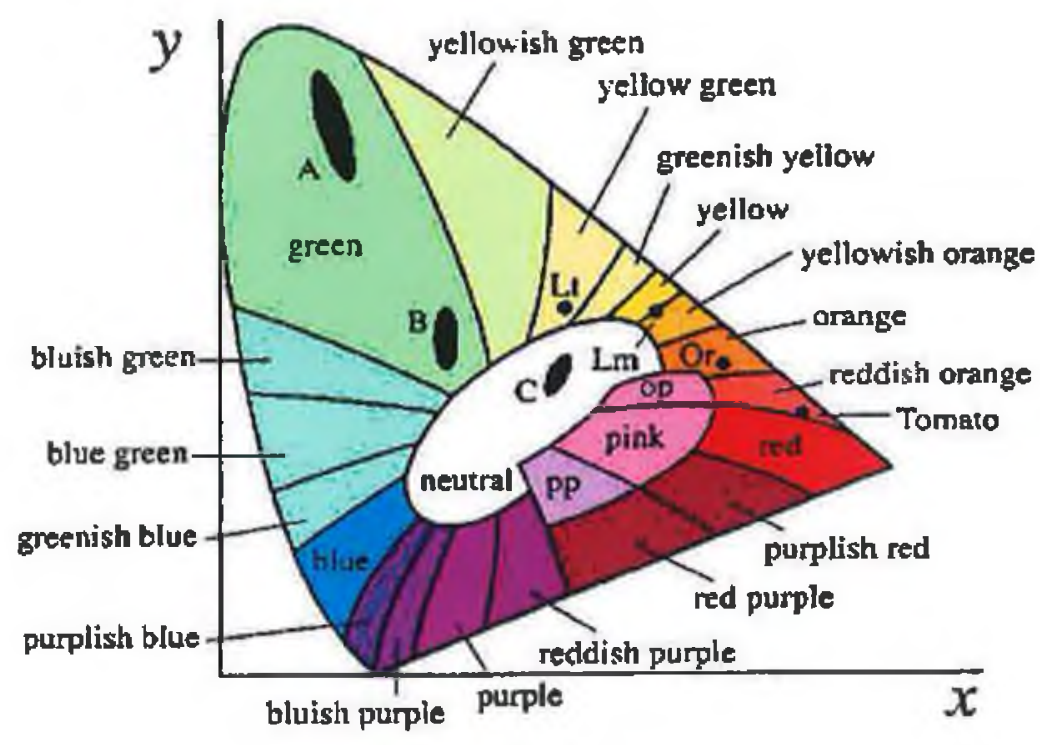

Figure B.3: CIE chromaticity diagram [99]

The colours are described by the luminance $\mathrm{Y}$ and the $\mathrm{x}, \mathrm{y}$ chromaticities. Any colour is specified by its trichromatic coefficients defined as:

$$
\begin{aligned}
& x=\frac{X}{X+Y+Z} \\
& y=\frac{Y}{X+Y+Z} \\
& z=\frac{Z}{X+Y+Z}
\end{aligned}
$$

where

$$
x+y+z=1
$$

The XYZ representation can describe any colour and the spectral composition corresponds to the colour matching characteristic of human visual systems, Figure B.3. The advantage of CIE-XYZ is that the result of an additive mixing between two colours will always lie on a line that connects the two colours in $\mathrm{x}-\mathrm{y}$ plane. The disadvantage is the non-uniformity of the CIE-XYZ plane [99]. 


\section{B.7 CIE-LUV Space}

CIE defined two new colour spaces, CIE-LUV and CIE-LAB to get more uniform and accurate models. The first of these two colour spaces is the CIE-LUV whose components are $\mathrm{L}, \mathbf{u}$ and $\mathrm{v}$. In CIE-LUV space the $\mathrm{L}$ component defines the luminancy, and $\mathrm{u}, \mathrm{v}$ components define the chrominance. This colour space is a perceptually uniform space in which two colours that are equidistant in the colour space are equally distant in perception. This means that the Euclidean distance between two colours in the CIE-LUV colour space is strongly correlated with the human perception. The CIE-LUV space is device independent, and the colours with the same LUV components look similar on all monitors. It is suitable for applications dealing with additive mixtures of coloured light such as the lighting and colour television industries. The CIE-LUV colour space is derived from CIE-XYZ as follows:

$$
\begin{aligned}
& L=116 *\left\{\frac{Y}{Y_{n}}\right\}^{\frac{1}{3}}-16 \quad \text { Luminance } \\
& u=13 * L\left(u^{*}-u_{n}^{*}\right) \text { red }- \text { green component } \\
& v=13 * L\left(v^{*}-v_{n}^{*}\right) \quad \text { yellow }- \text { blue component } \\
& u^{*}=\frac{4 X}{X+15 Y+3 Z} \\
& v^{*}=\frac{9 Y}{X+15 Y+3 Z}
\end{aligned}
$$

where the subscript $n$ represents white point measurement and $\mathrm{u}, \mathrm{v}$ are the uniform chromaticities. The white point is the chromaticity of the colour reproduced by equal red, green, and blue components. It is a function of the ratio of power among the primaries $(r, g, b)$. The perceptually linear colour difference formula between two colours is

$$
\Delta E_{L u v}=\sqrt{(\Delta L)^{2}+(\Delta u)^{2}+(\Delta v)^{2}}
$$

where, $\Delta$ is the difference between corresponding components. 


\section{B.8 CIE-LAB Space}

CIE-LAB is a popular colour space and it is based on the complementary colour pairs, which have been proven to be analogous to with the sensations generated by the retina. Colour pairings include red-green, blue-yellow and black-white, as in Figure B.4 These sensations are used to develop a system known as the

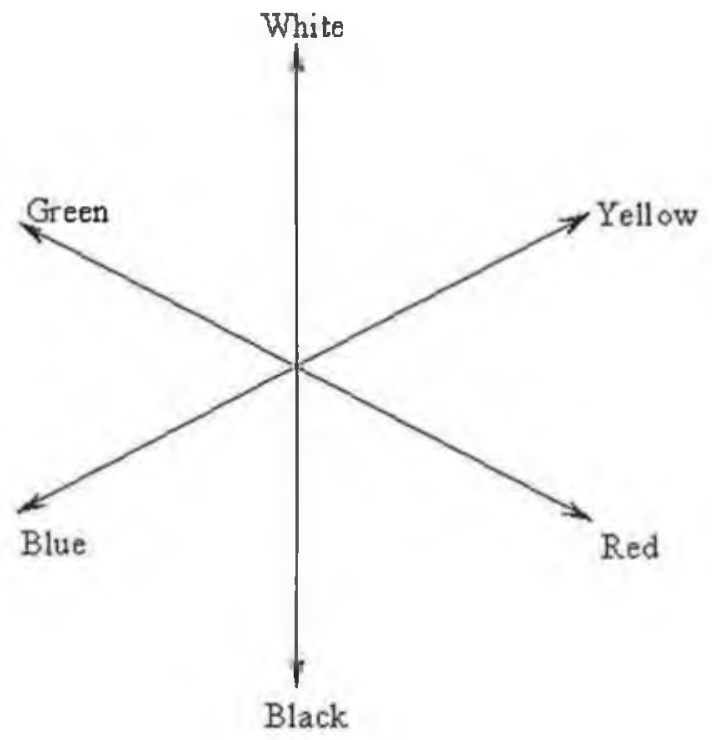

Figure B.4: Complementary colour pairs

complementary colour system. The CIE-LAB colour space is derived from CIE$\mathrm{XYZ}$ as follows:

$$
\begin{aligned}
& L=116 *\left\{\frac{Y}{Y_{n}}\right\}^{\frac{1}{3}}-16 \\
& a=500 *\left[\left\{\frac{X}{X_{n}}\right\}^{\frac{1}{3}}-\left\{\frac{Y}{Y_{n}}\right\}^{\frac{1}{3}}\right] \\
& b=500 *\left[\left\{\frac{Y}{Y_{n}}\right\}^{\frac{1}{3}}-\left\{\frac{Z}{Z_{n}}\right\}^{\frac{1}{3}}\right]
\end{aligned}
$$

where $n$ stands for the white point representation. The CIE-LAB colour space has many advantages. The perceptually linear colour difference formula between 
two colours is

$$
\Delta E_{L a b}=\sqrt{(\Delta L)^{2}+(\Delta a)^{2}+(\Delta b)^{2}}
$$

where, $\Delta$ is the difference between corresponding components. A key advantage of this representation is the fact that it is a perceptually equalised colour space, i.e., numerical distance in this colour space is proportional to perceived colour difference [99]. Above all, it is not dependent on any particular device. The main difference between the two colour spaces defined by CIE is in the chromatic adaptation model implemented. The CIE-LAB colour space normalises its values by the division with the white point while the CIE-LUV colour space normalises with values by the subtraction of the white point. 


\section{Appendix C}

\section{Implementation}

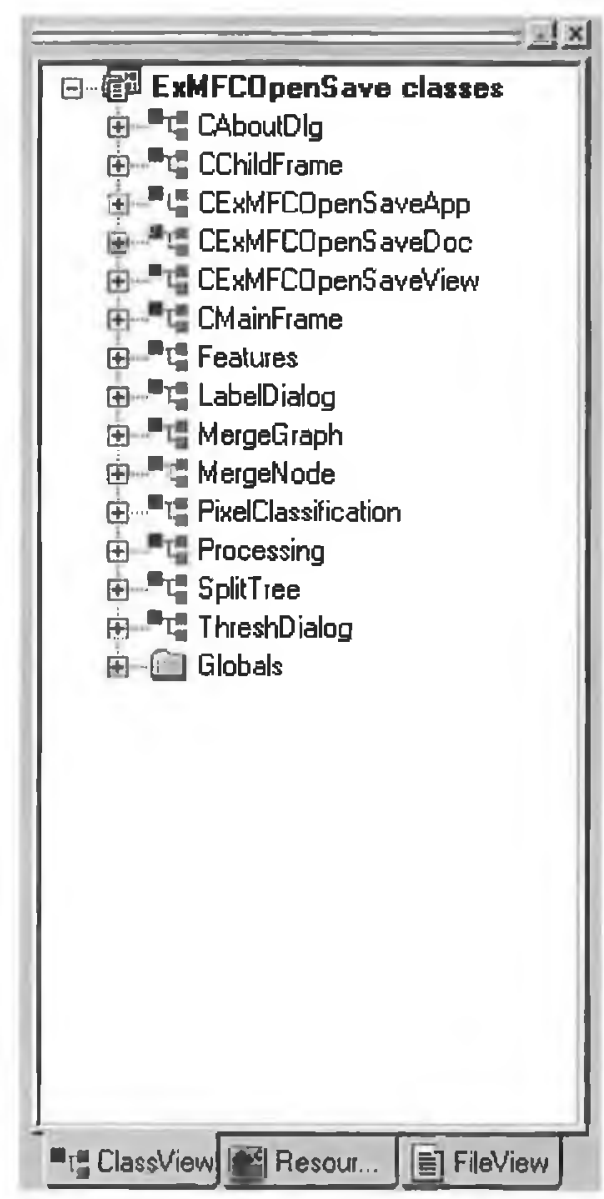

Figure C.1: Different classes used in Method-II 


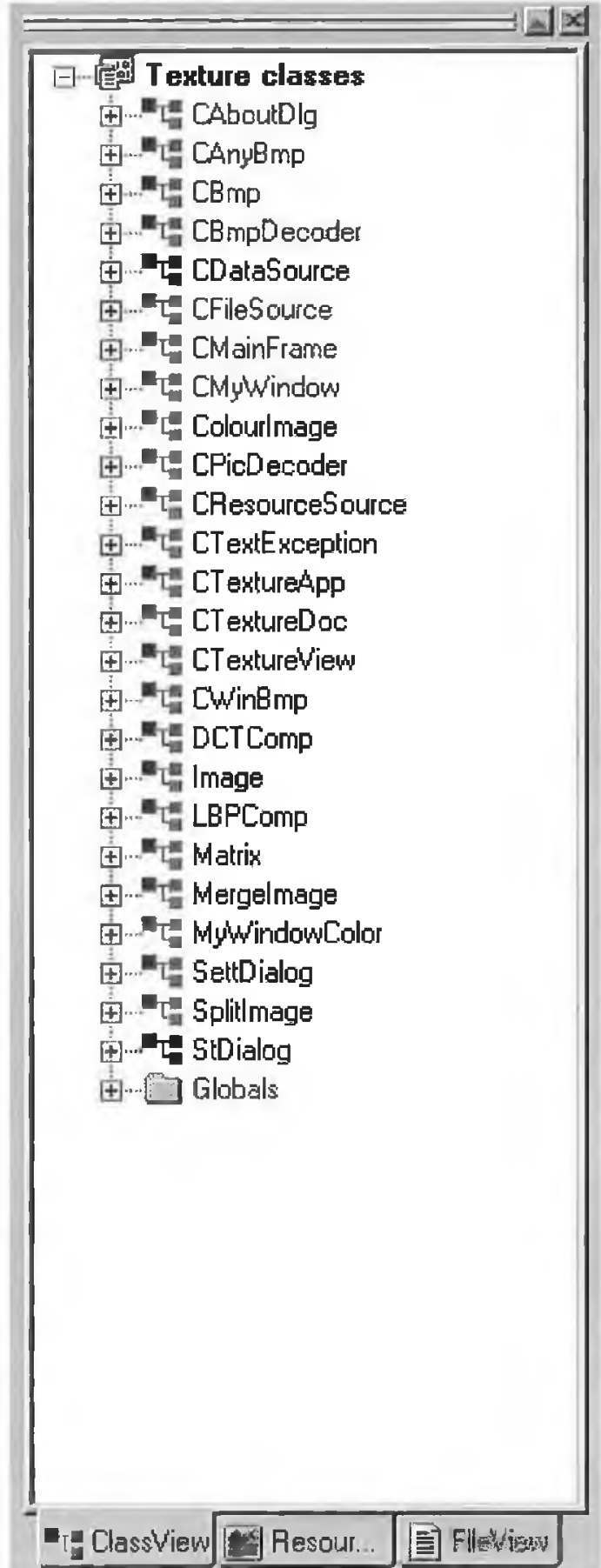

Figure C.2: Different classes used in Method-III 


\section{Appendix D}

\section{Database Images}

\section{D.1 Database of Mosaic Images}

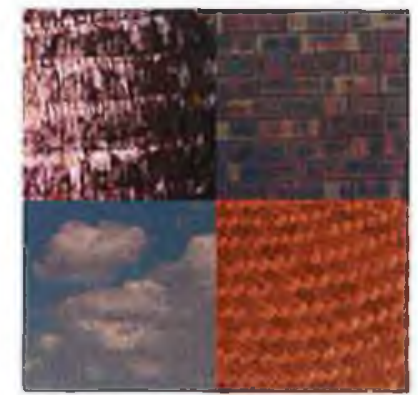

(M1)

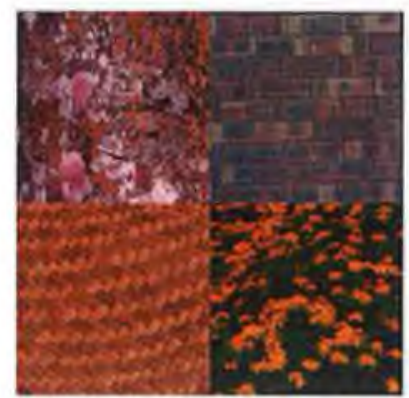

(M4)

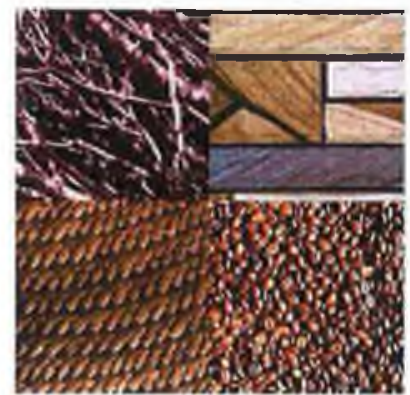

(M2)

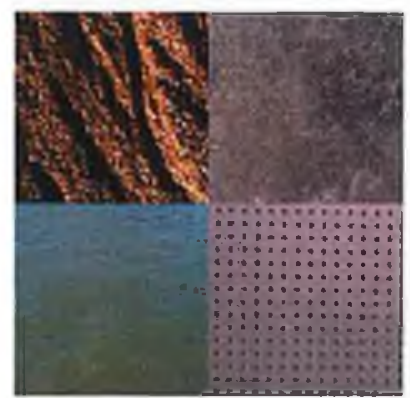

(M5)

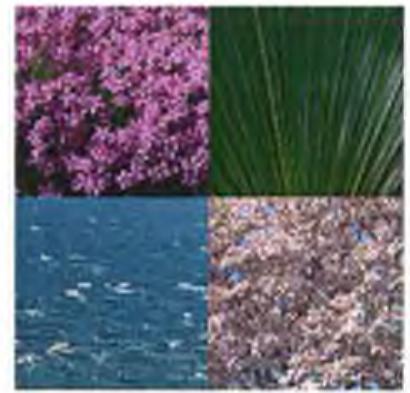

(M3)

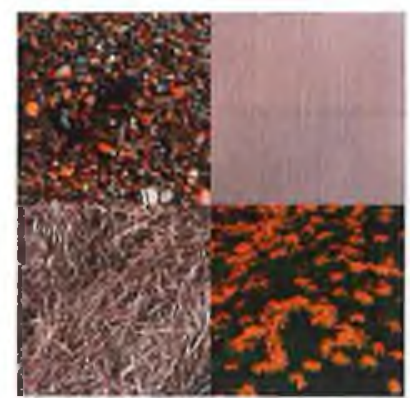

(M6)

Figure D.1: M1-M6 are the mosaic images constructed from VisTex database 


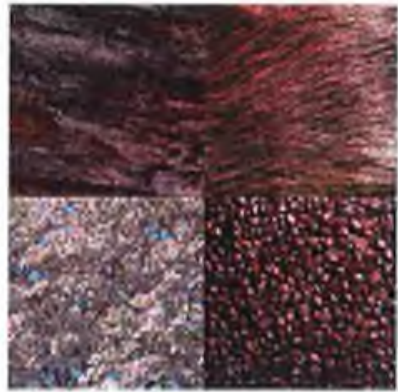

(M7)

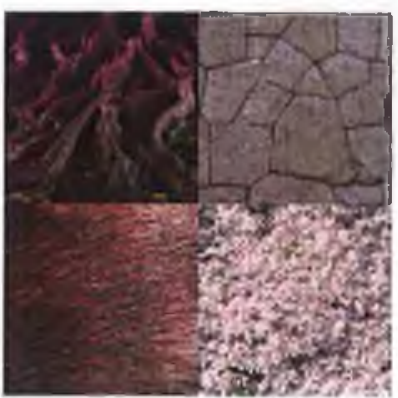

(M10)

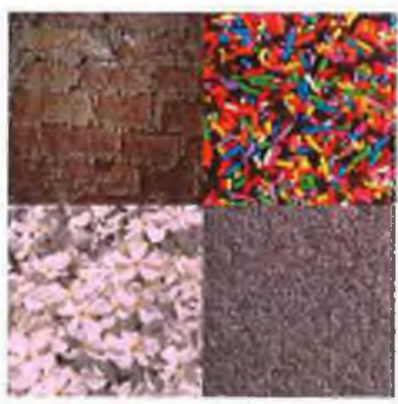

(M13)

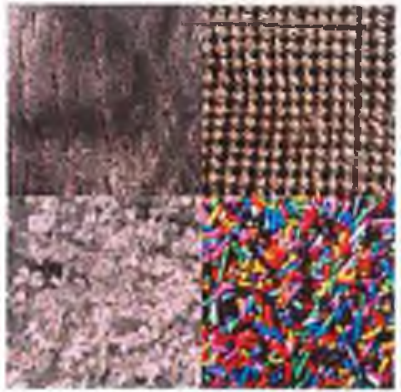

(M16)

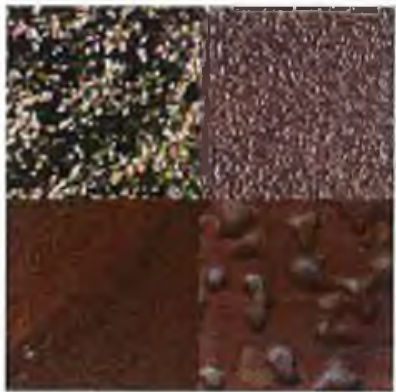

(M8)

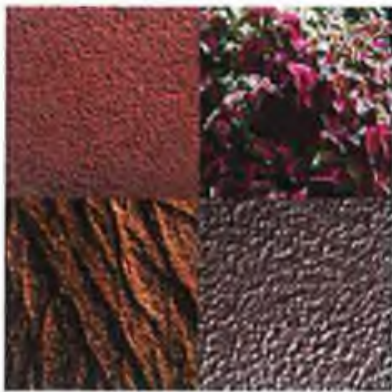

(M11)

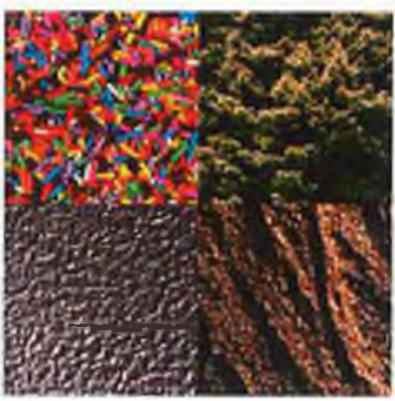

(M14)

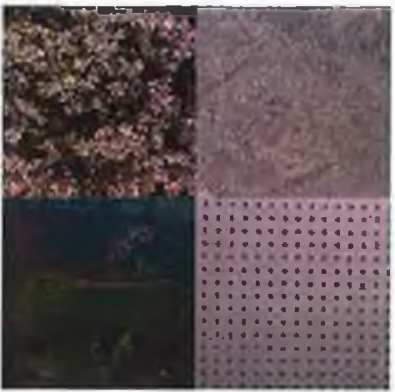

(M17)

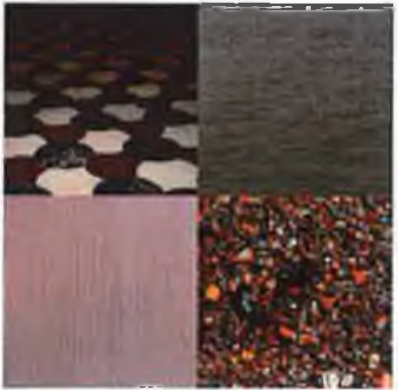

(M9)

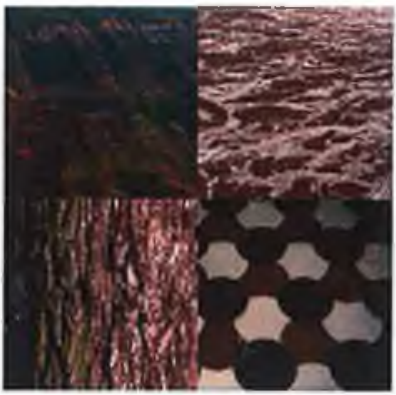

(M12)

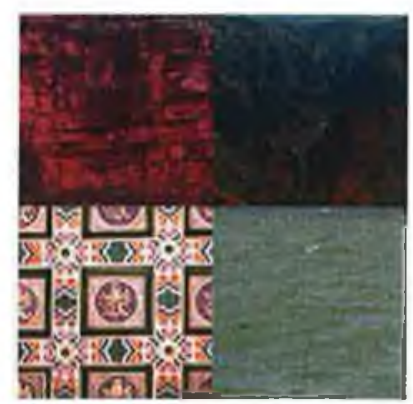

(M15)

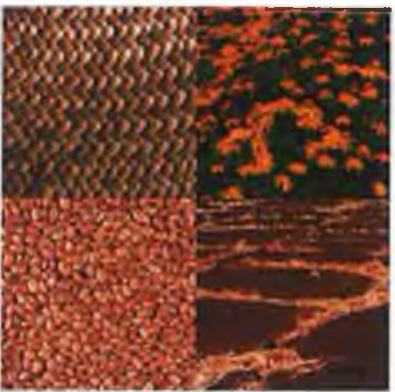

(M18)

Figure D.2: M7-M18 are the mosaic images constructed from VisTex database 191 


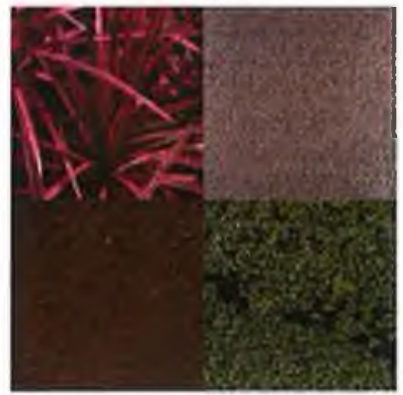

(M19)

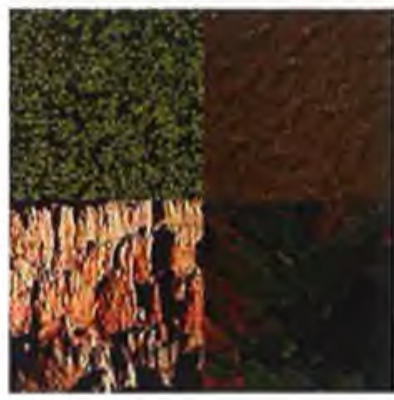

(M22)

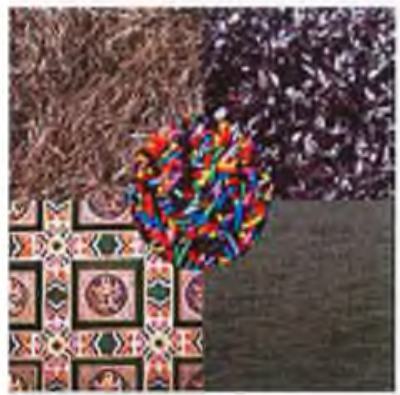

(M25)

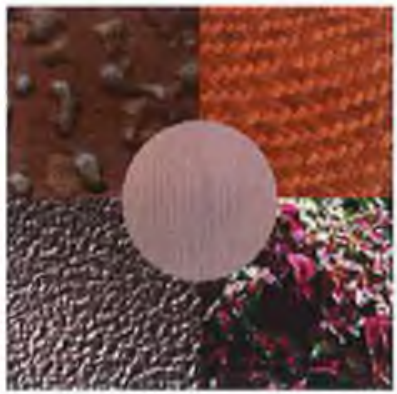

(M28)

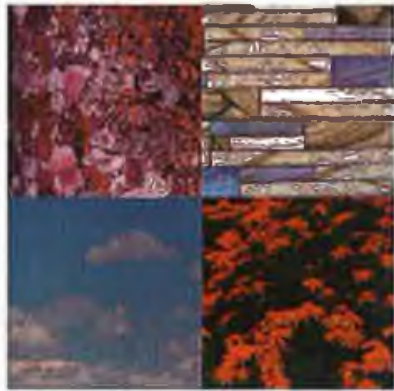

(M20)

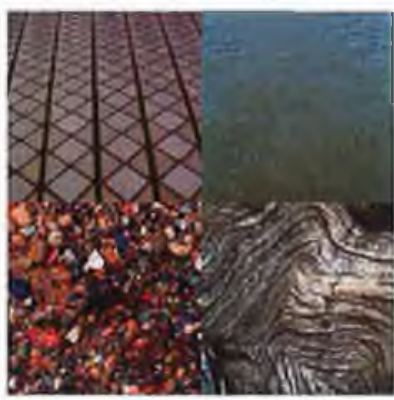

(M23)

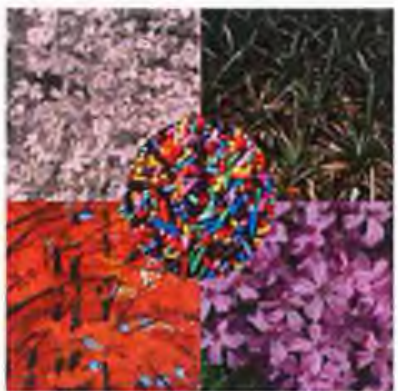

(M26)

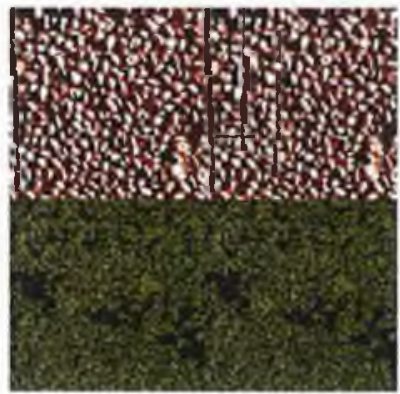

(M29)

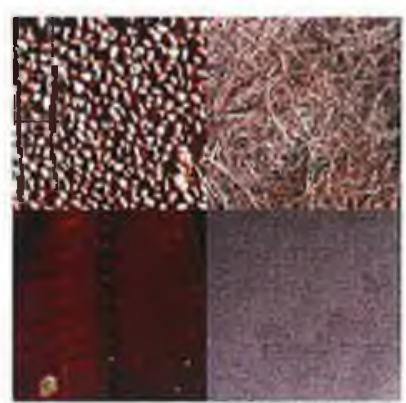

(M21)

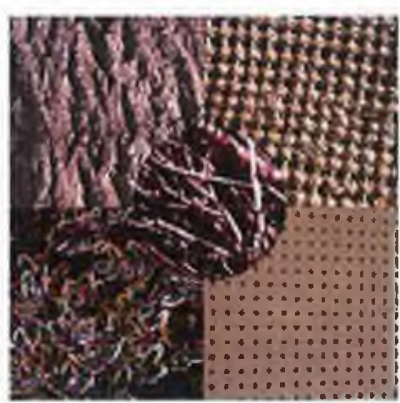

(M24)

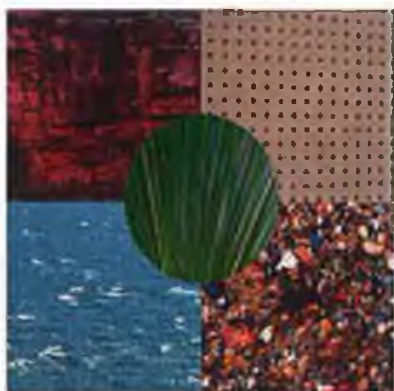

(M27)

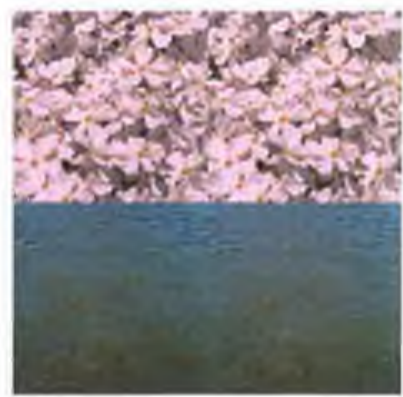

(M30)

Figure D.3: M19-M30 are the mosaic images constructed from VisTex database 


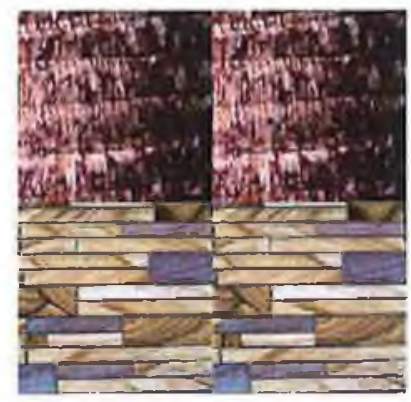

(M31)

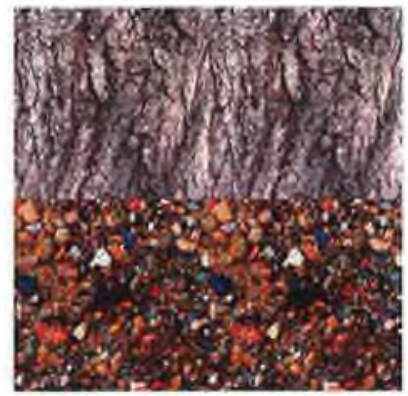

(M34)

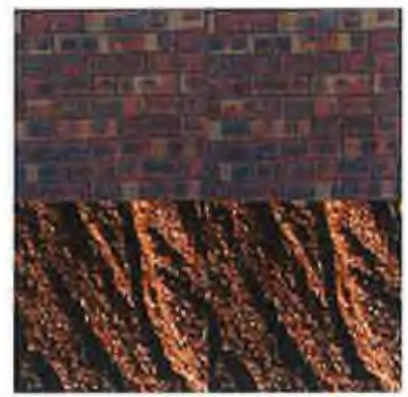

(M37)

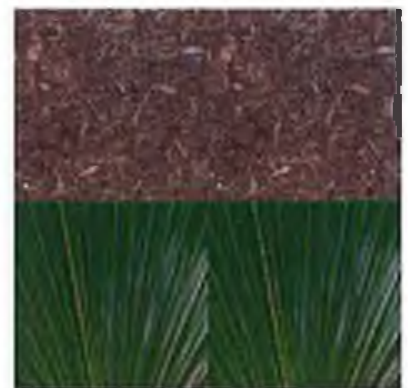

(M32)

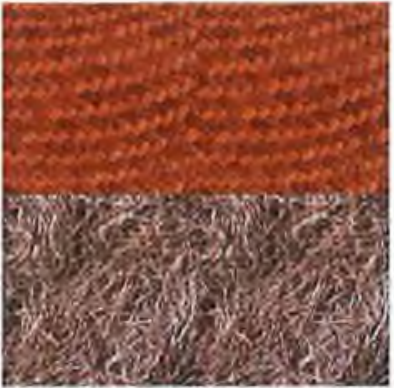

(M35)

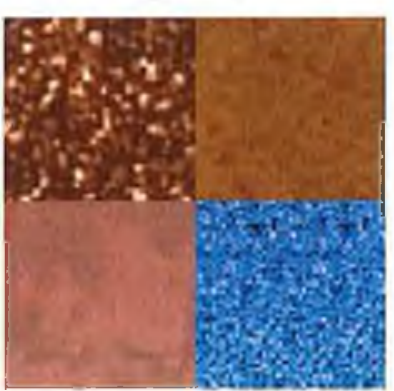

(M38)

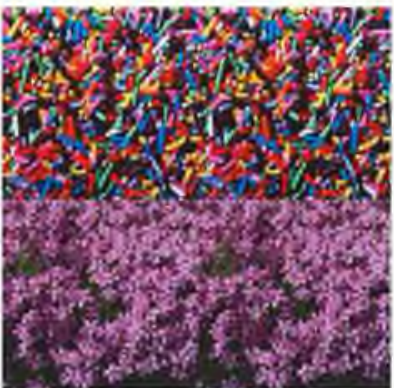

(M33)

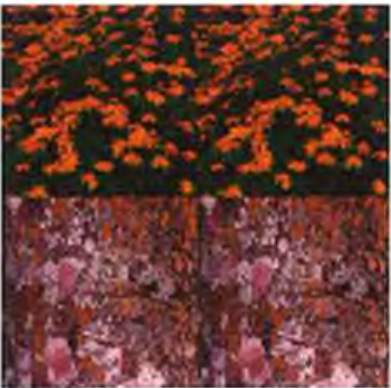

(M36)

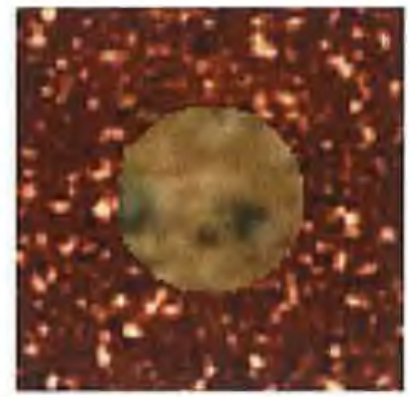

(M39)

Figure D.4: M31-M37 are the mosaic images from VisTex database, M38 and M39 were mosaic images used by Mirmehdi and Petrou [86] 


\section{D.2 Database of Natural Images}

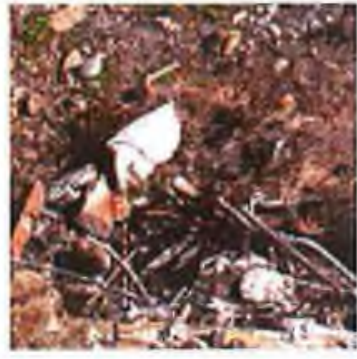

(N1)

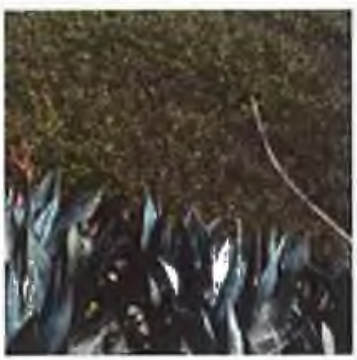

(N4)

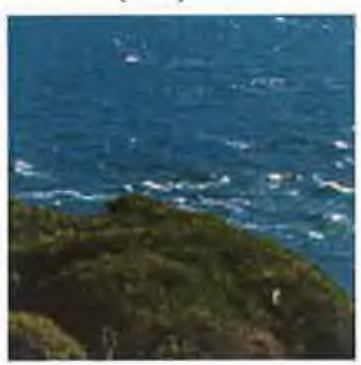

(N7)

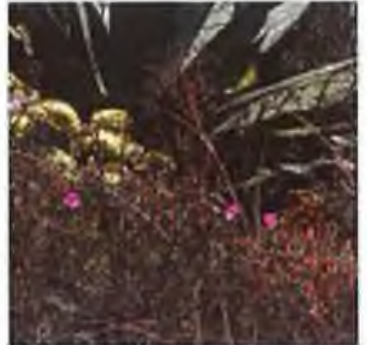

(N2)

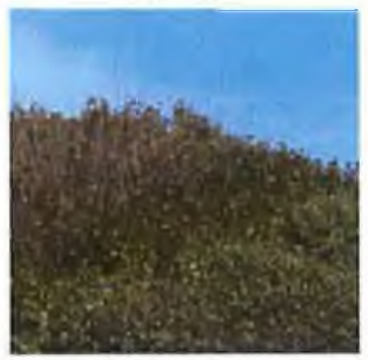

(N5)

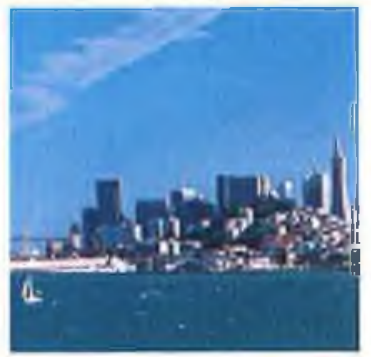

(N8)

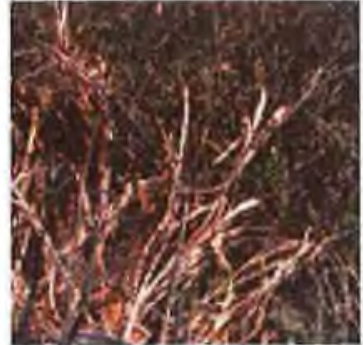

(N3)

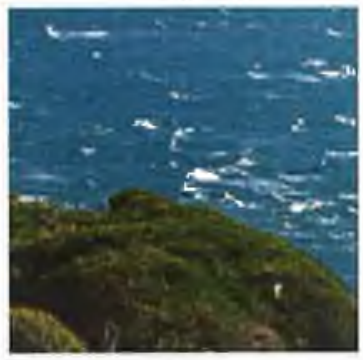

(N6)

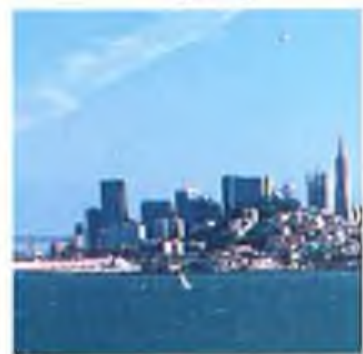

(N9)

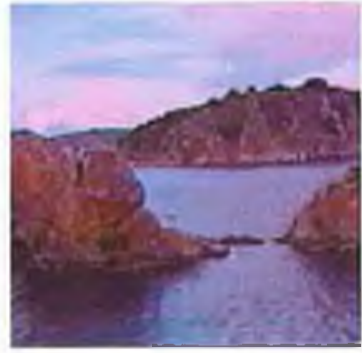

(N10)

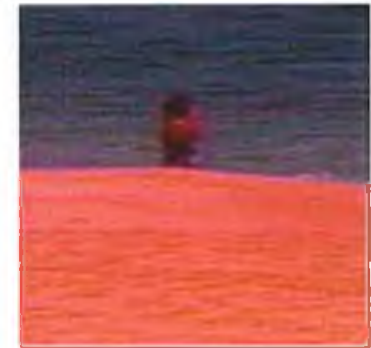

(N11)

Figure D.5: N1-N9 are the natural images from VisTex database, N10 and N11 are the natural images which were used by Panjwani and Healey [24] 


\section{Appendix E}

\section{Results - Method-I}

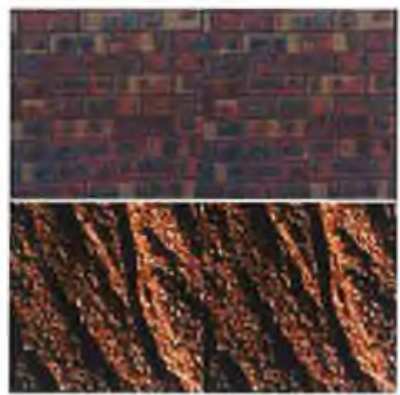

(MI-LBP-5)

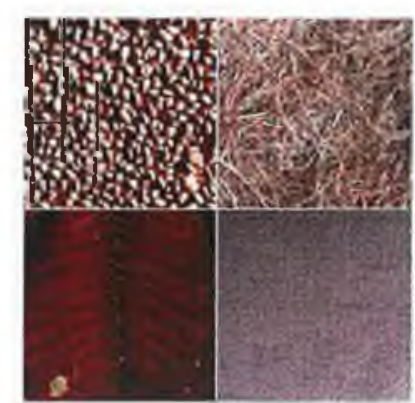

(MI-LBP-8)

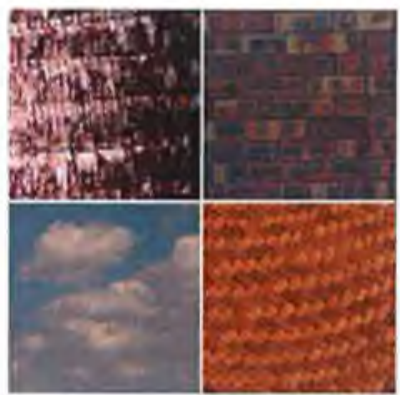

(MI-LBP-6)

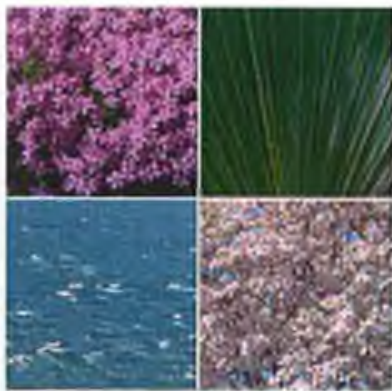

(MI-LBP-9)

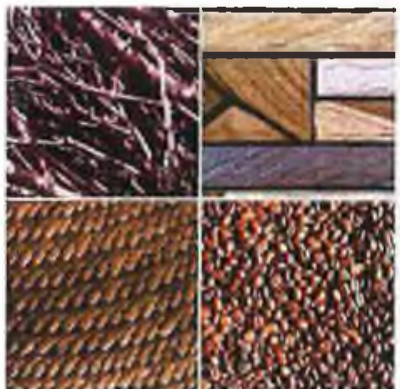

(MI-LBP-7)

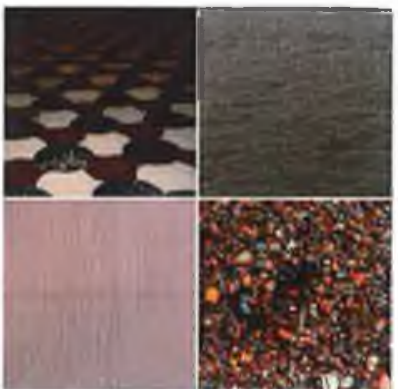

(MI-LBP-10)

Figure E.1: Sample segmented results of mosaic images from Method-I using $\mathrm{LBP} / \mathrm{C}$ in different colour planes 


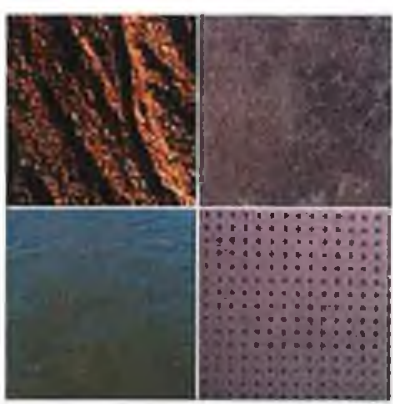

(MI-LBP-11)

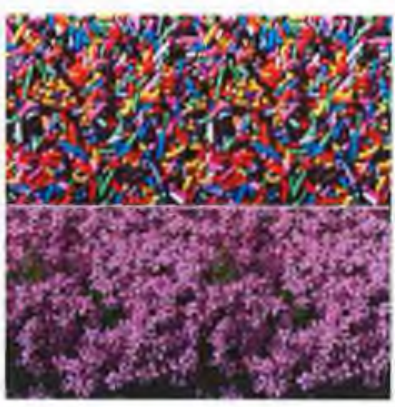

(MI-LBP-14)

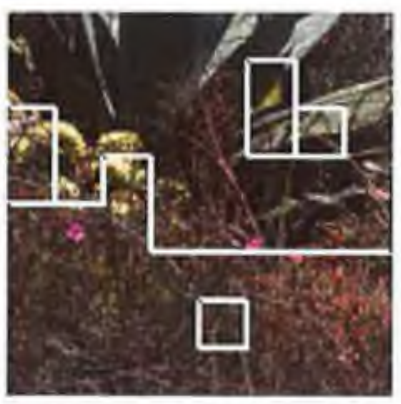

(MI-LBP-N1)

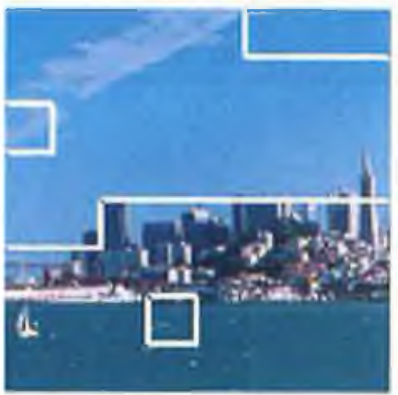

(MI-LBP-N4)

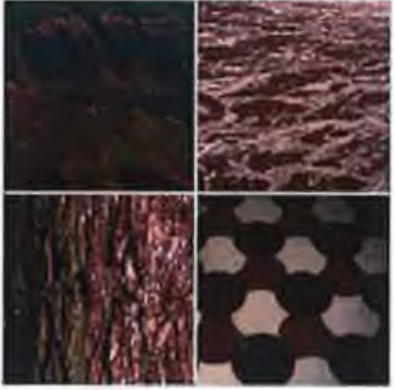

(MI-LBP-12)

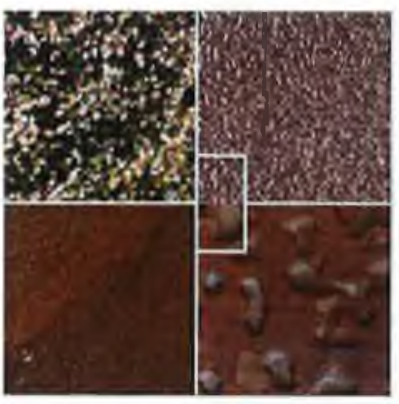

(MI-LBP-15)

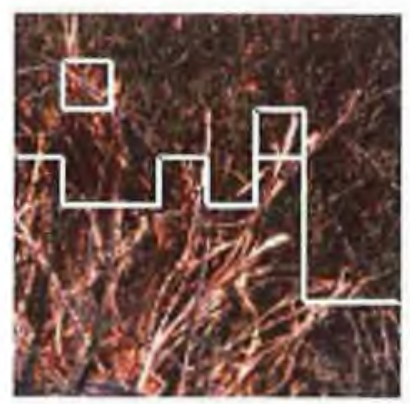

(MI-LBP-N2)

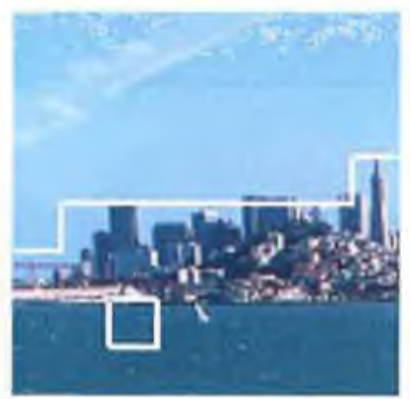

(MI-LBP-N5)

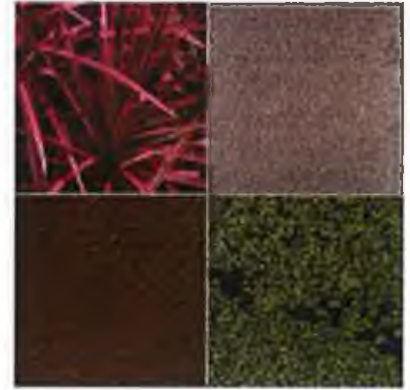

(MI-LBP-13)

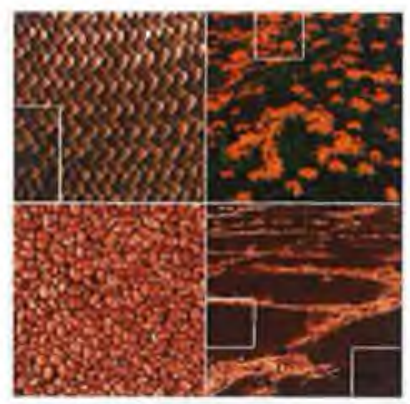

(MI-LBP-16)

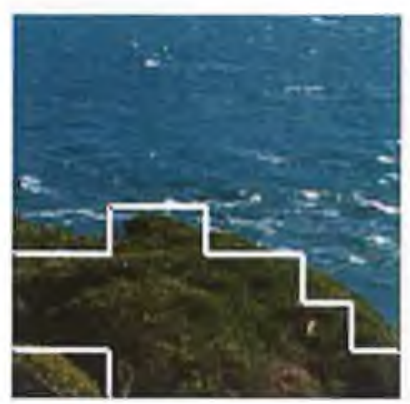

(MI-LBP-N3)

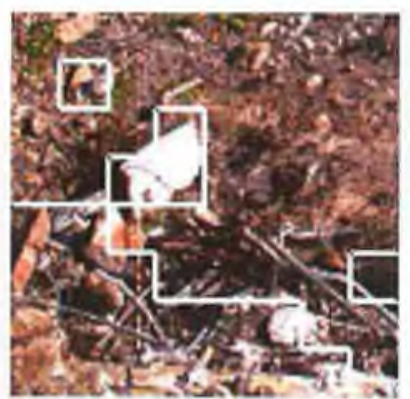

(MI-LBP-N6)

Figure E.2: Sample segmented results of mosaic and natural images from Method-I using LBP/C in different colour planes 


\section{Appendix F}

\section{Results - Method-II}

\section{F.1 Results using LBP/C and colour features}

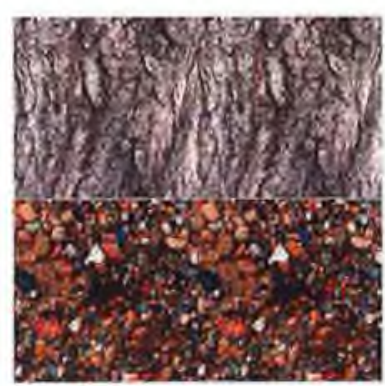

(MII-LBP-5)

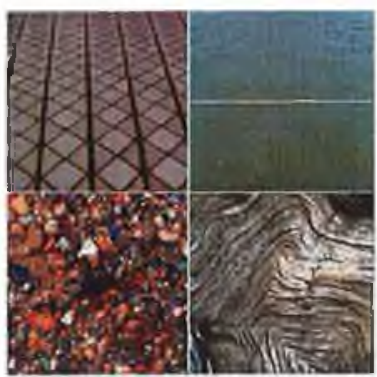

(MII-LBP-8)
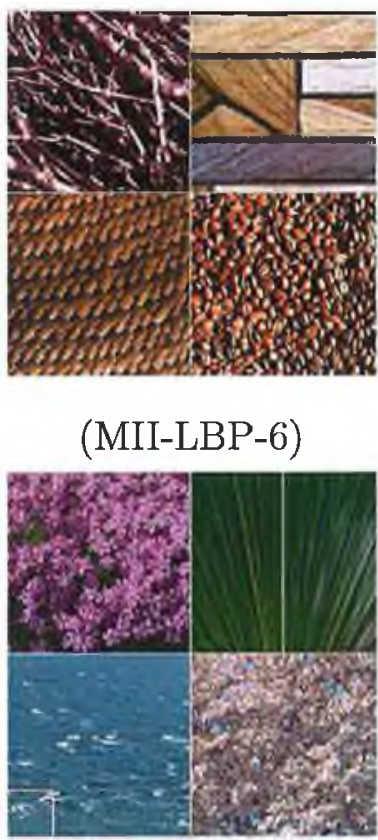

(MII-LBP-9)

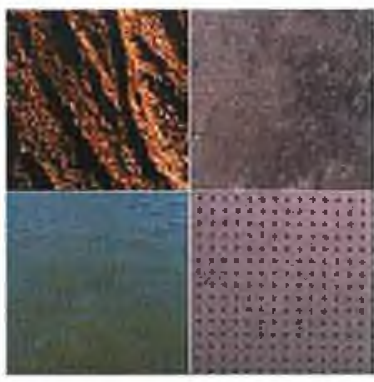

(MII-LBP-7)

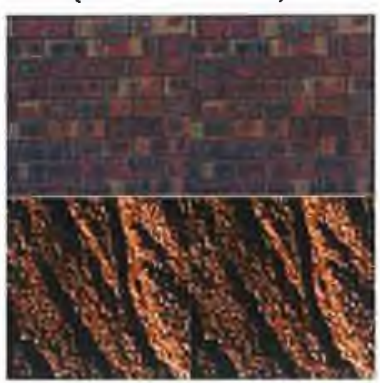

(MII-LBP-10)

Figure F.1: Sample segmented results from Method-II using LBP/C and colour features 


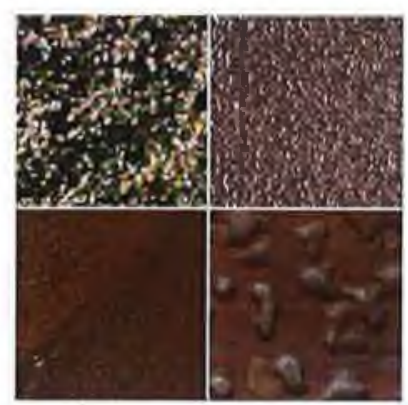

(MII-LBP-11)

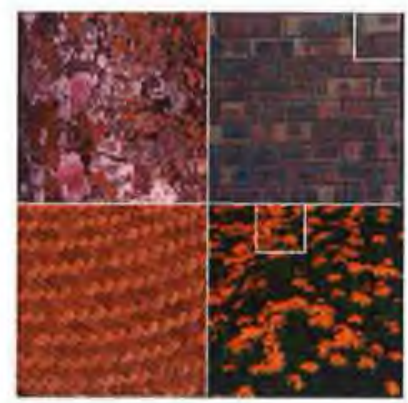

(MII-LBP-14)

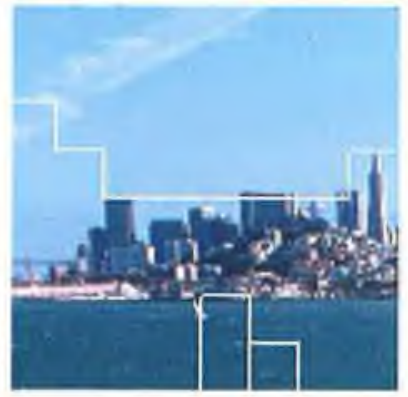

(MII-LBP-N3)

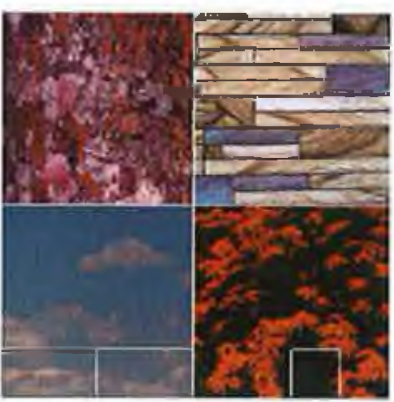

(MII-LBP-12)

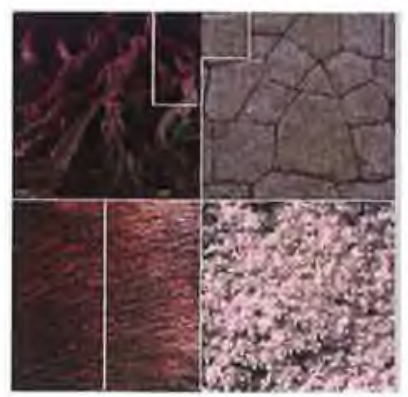

(MII-LBP-15)

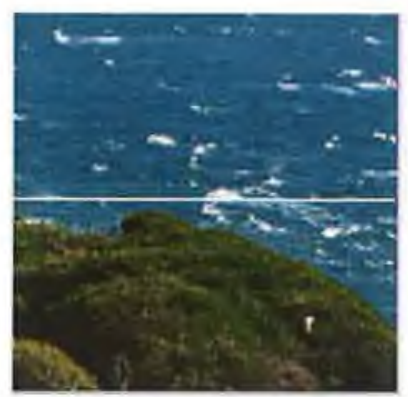

(MII-LBP-N4)

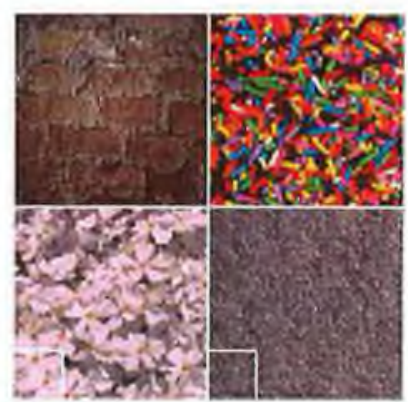

(MII-LBP-13)

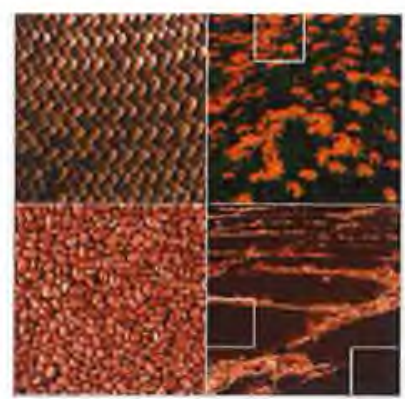

(MII-LBP-16)

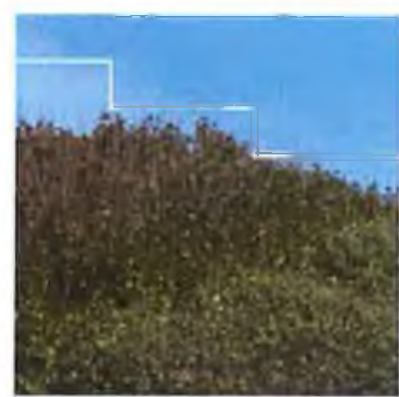

(MII-LBP-N5)

Figure F.2: Sample segmented results from Method-II using LBP/C and colour features 


\section{F.2 Results using DCT and colour features}

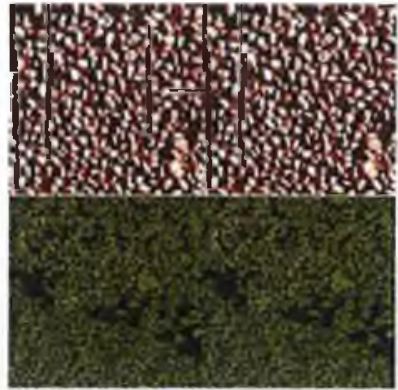

(MII-DCT-5)

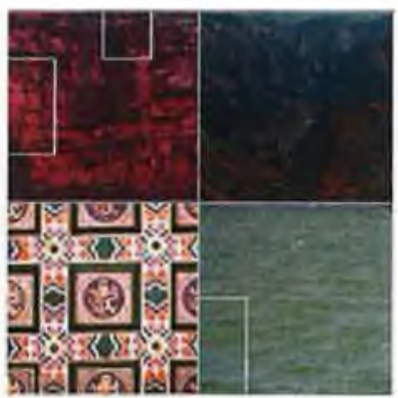

(MII-DCT-8)

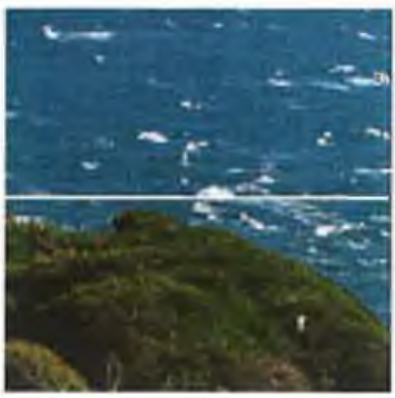

(MII-DCT-N1)

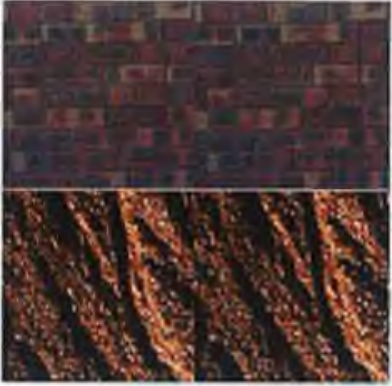

(MII-DCT-6)

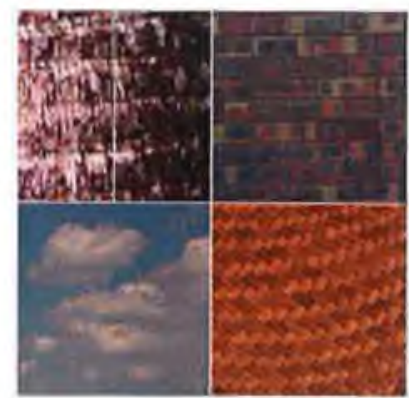

(MII-DCT-9)

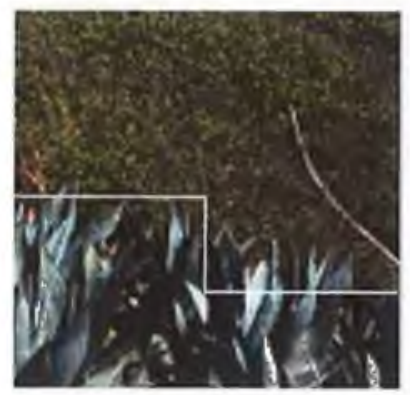

(MII-DCT-N2)

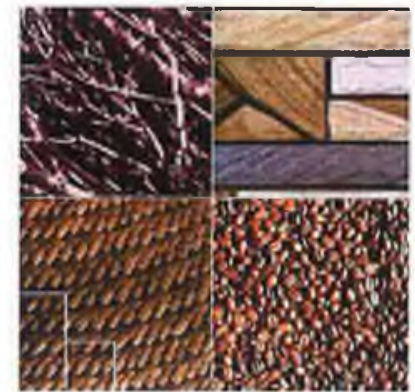

(MII-DCT-7)

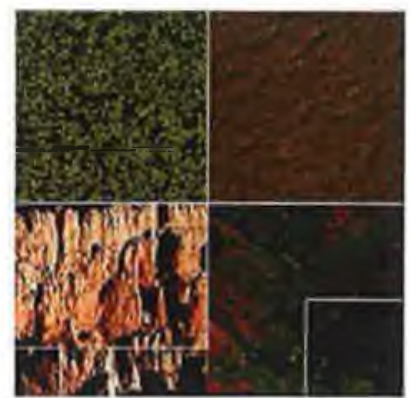

(MII-DCT-10)

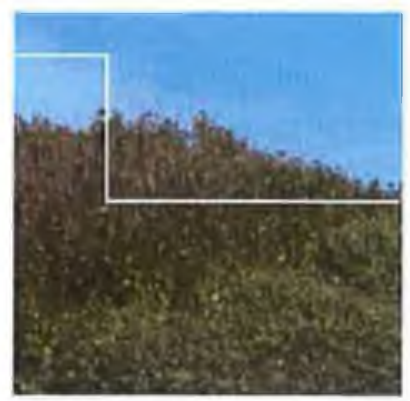

(MII-DCT-N3)

Figure F.3: Sample segmented results from Method-II using DCT and colour features 


\section{Appendix G}

\section{Results - Method-III}

G.1 Results using LBP/C with colour clustering
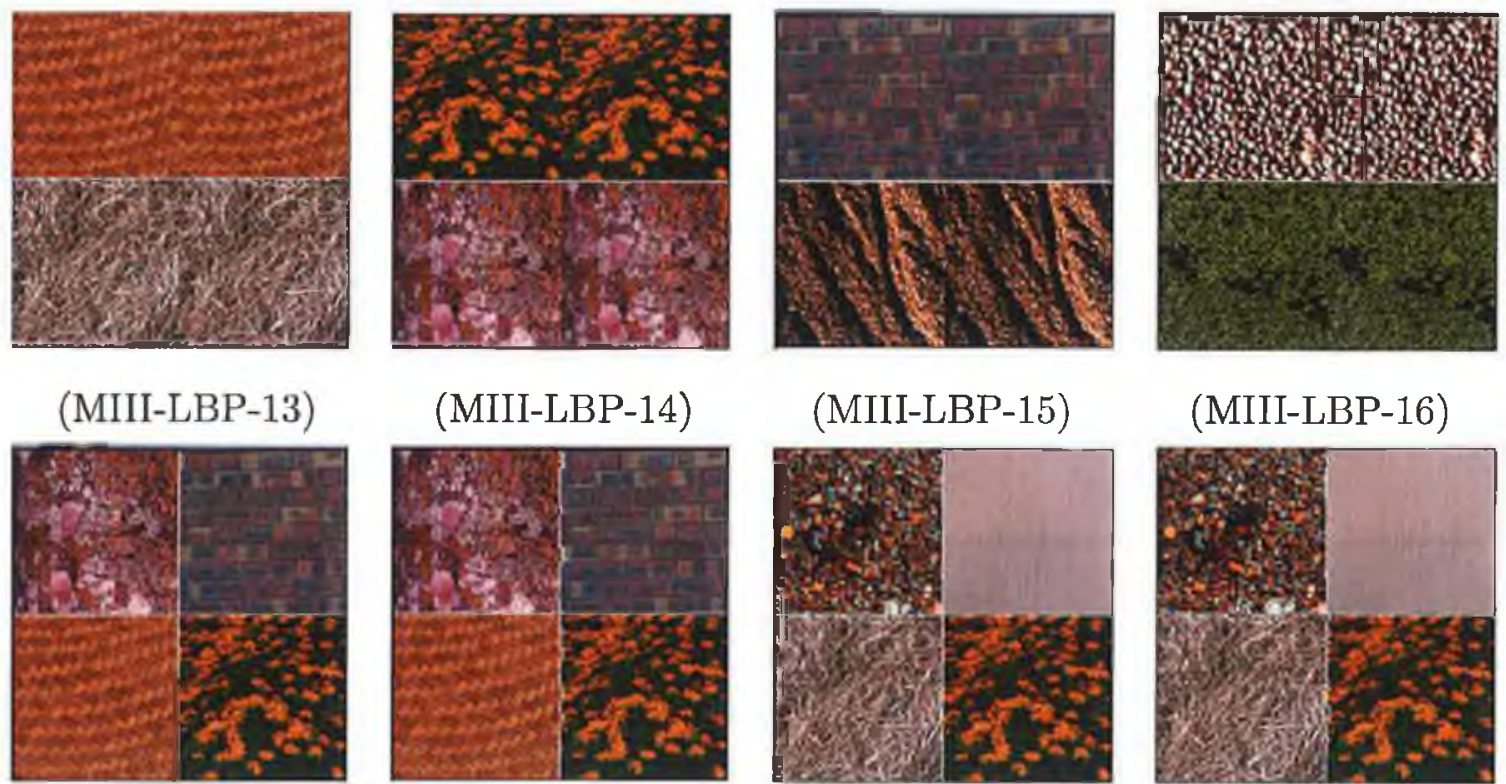

(MIII-LBP-16)

(MIII-LBP-17a)

(MIII-LBP-17b)

(MIII-LBP-18a)

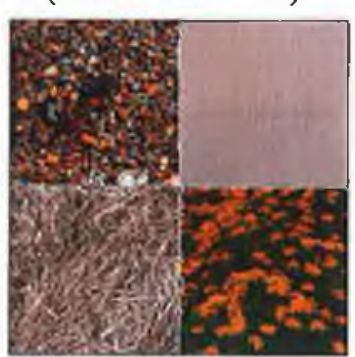

(MIII-LBP-18b)

Figure G.1: Sample segmented results from Method-III using LBP/C with colour clustering 


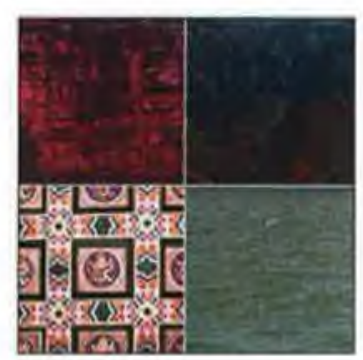

(MIII-LBP-19a)

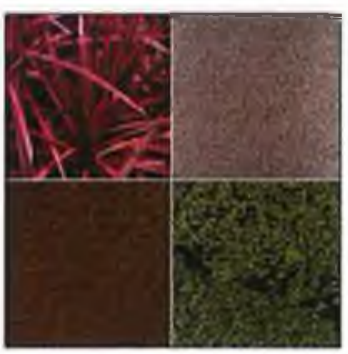

(MIII-LBP-21a)

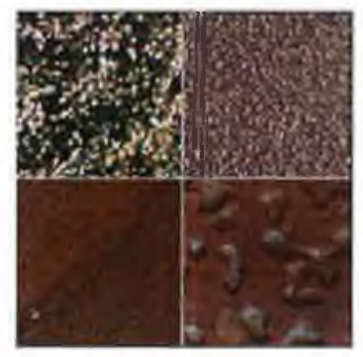

(MIII-LBP-23a)

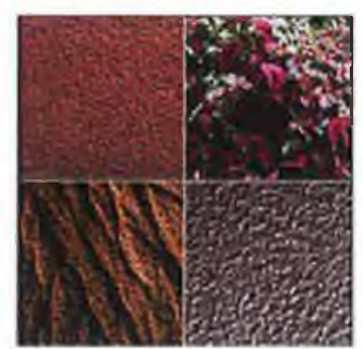

(MIII-LBP-25a)

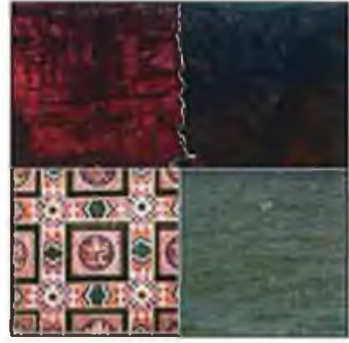

(MIII-LBP-19b)

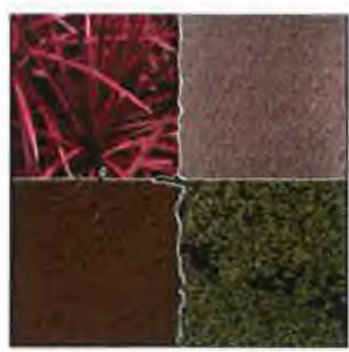

(MIII-LBP-21b)

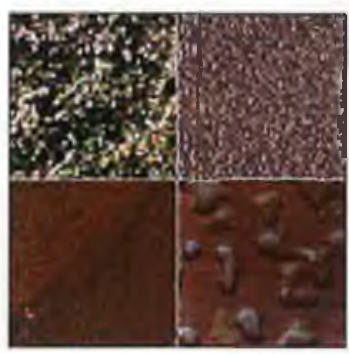

(MIII-LBP-23b)

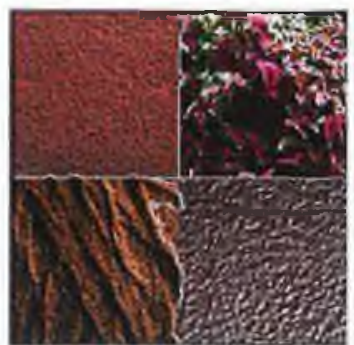

(MIII-LBP-25b)

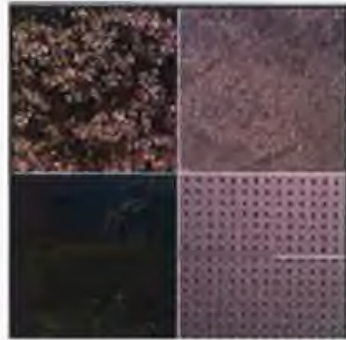

(MIII-LBP-20a)

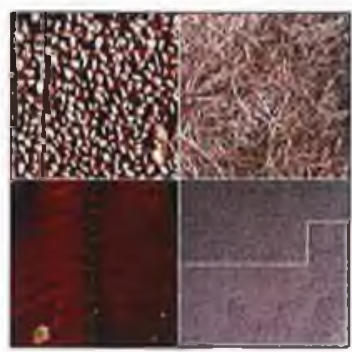

(MIII-LBP-22a)

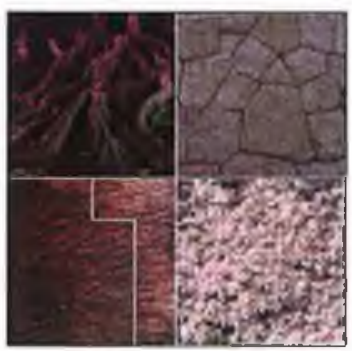

(MIII-LBP-24a)

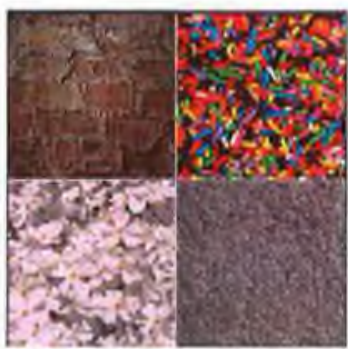

(MIII-LBP-26a)

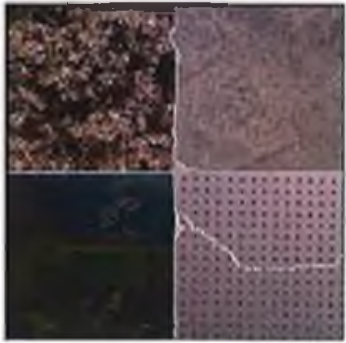

(MIII-LBP-20b)

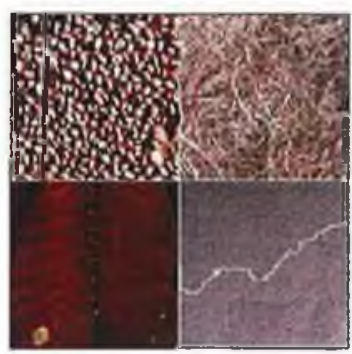

(MIII-LBP-22b)

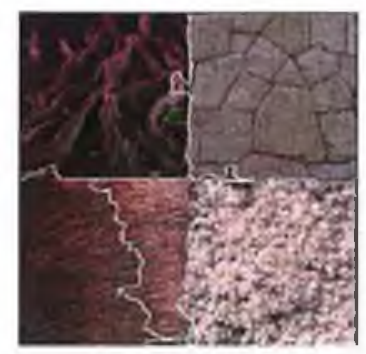

(MIII-LBP-24b)

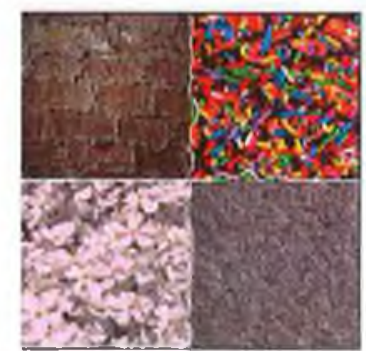

(MIII-LBP-26b)

Figure G.2: Sample segmented results from Method-III using LBP/C with colour clustering 


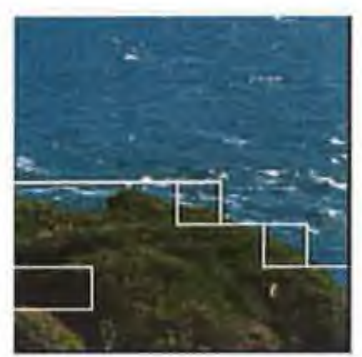

(MIII-LBP-N4a)

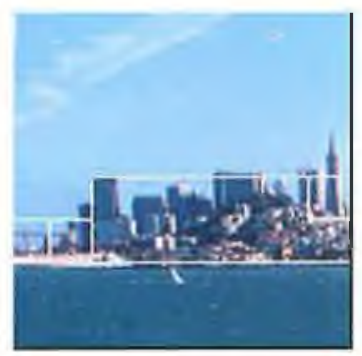

(MIII-LBP-N6a)

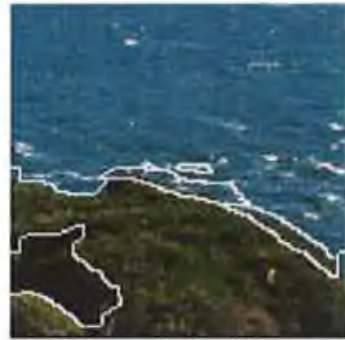

(MIII-LBP-N4b)

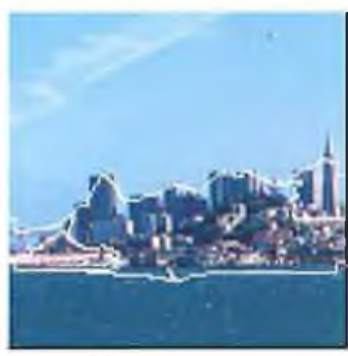

(MIII-LBP-N6b)

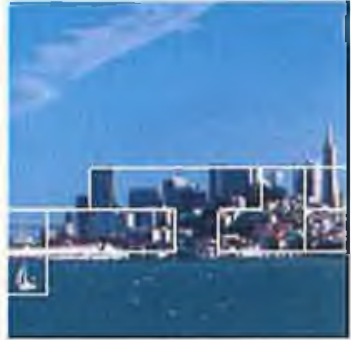

(MIII-LBP-N5a)

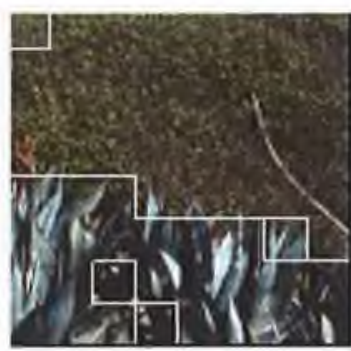

(MIII-LBP-N7a)

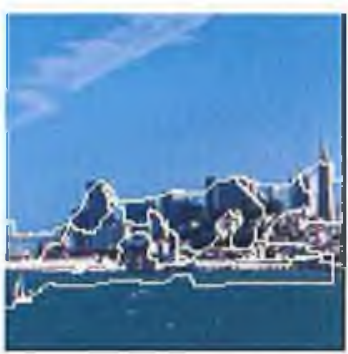

(MIII-LBP-N5b)

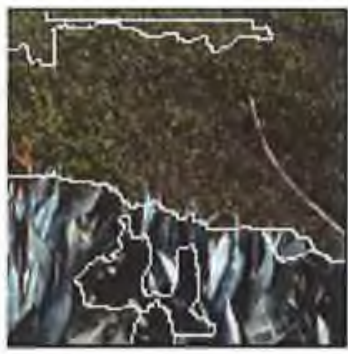

(MIII-LBP-N7b)

Figure G.3: Sample segmented results of natural images from Method-III using $\mathrm{LBP} / \mathrm{C}$ with colour clustering

\section{G.2 Results using DCT with colour clustering}

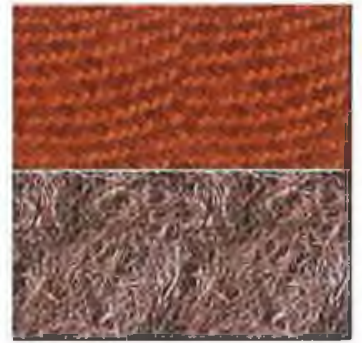

(MIII-DCT-5)

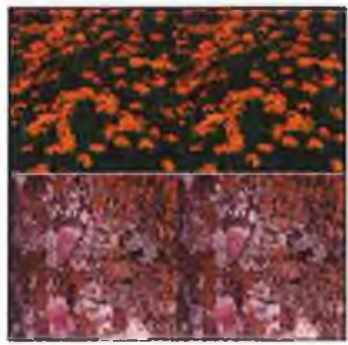

(MIII-DCT-6)

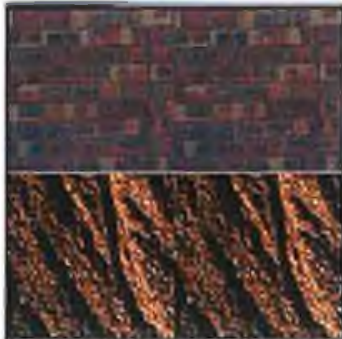

(MIII-DCT-7)

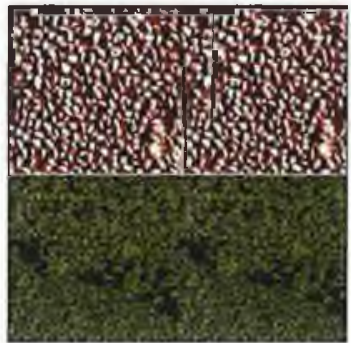

(MIII-DCT-8)

Figure G.4: Sample segmented results from Method-III using DCT with colour clustering 


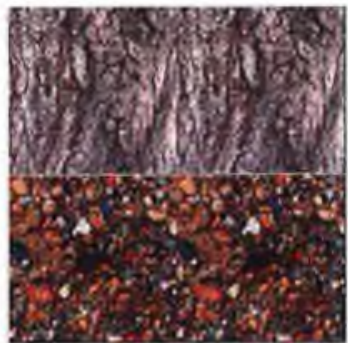

(MIII-DCT-9)

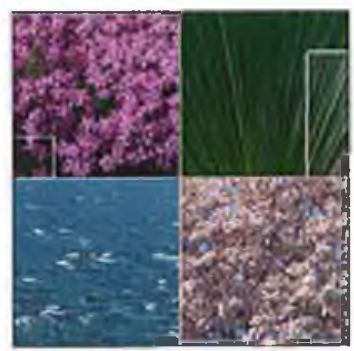

(MIII-DCT-12a)

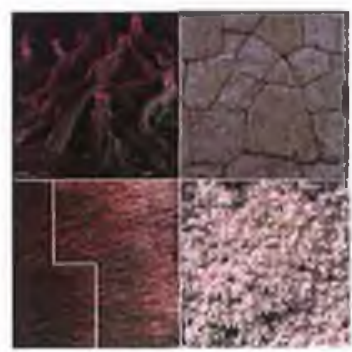

(MIII-DCT-14a)

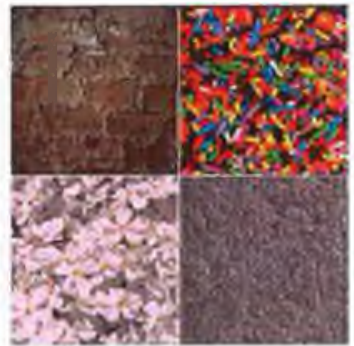

(MIII-DCT-16a)

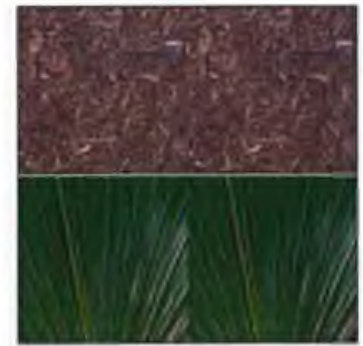

(MIII-DCT-10)

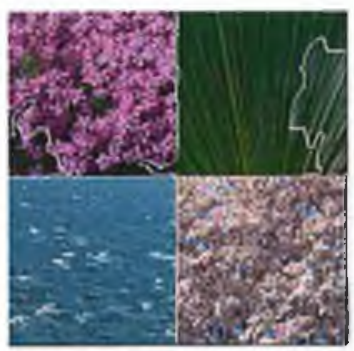

(MIII-DCT-12b)

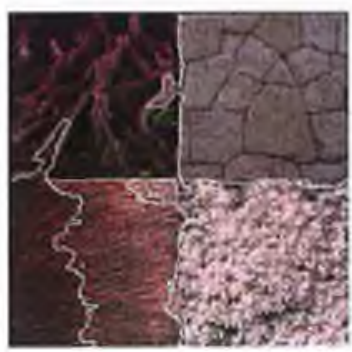

(MIII-DCT-14b)

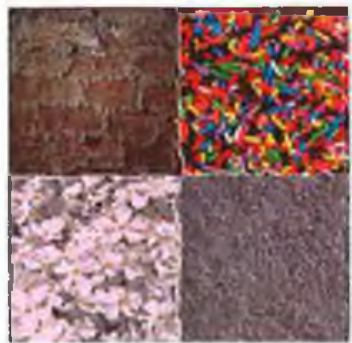

(MIII-DCT-16b)

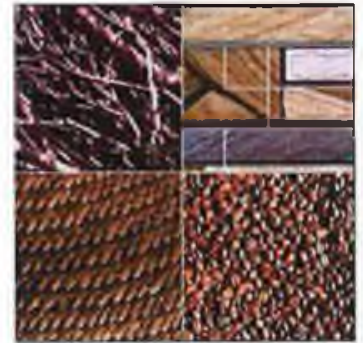

(MIII-DCT-11a)

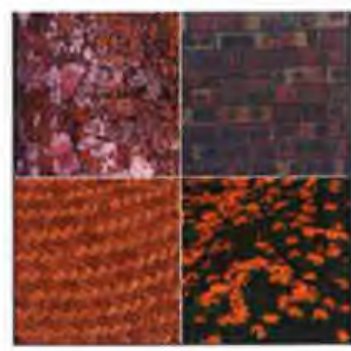

(MIII-DCT-13a)

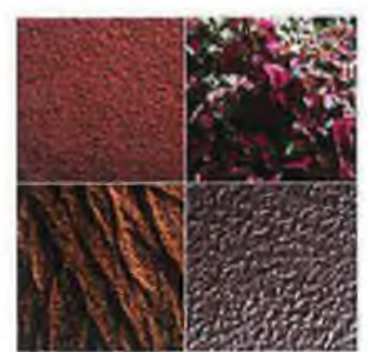

(MIII-DCT-15a)

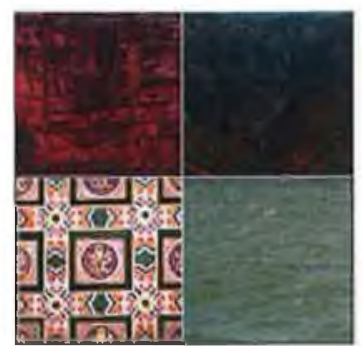

(MIII-DCT-17a)

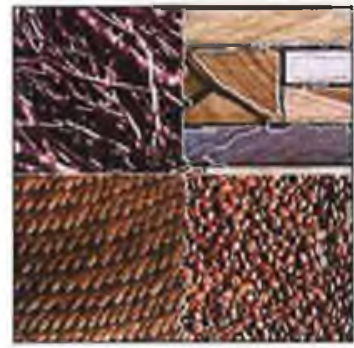

(MIII-DCT-11b)

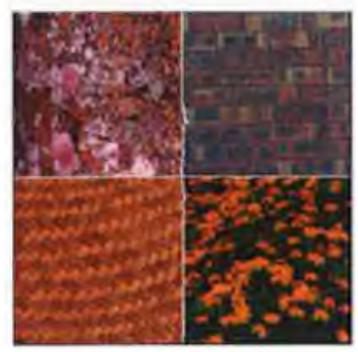

(MIII-DCT-13b)

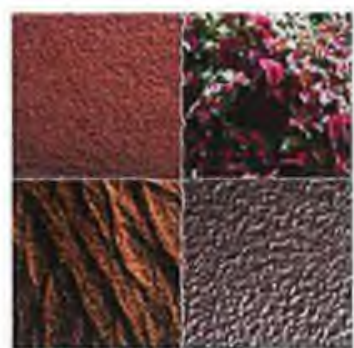

(MIII-DCT-15b)

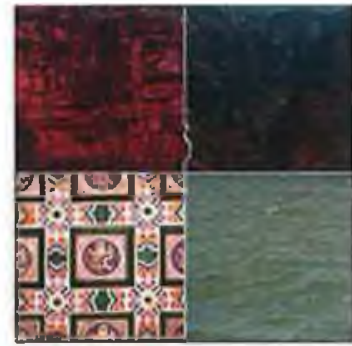

(MIII-DCT-17b)

Figure G.5: Sample segmented results from Method-III using DCT with colour clustering 


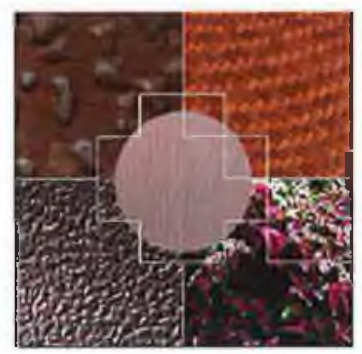

(MIII-DCT-18a)

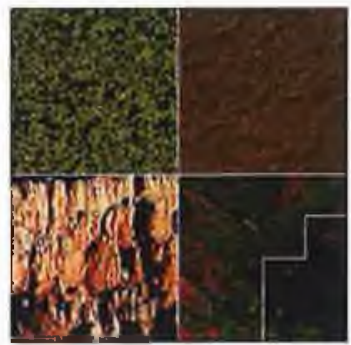

(MIII-DCT-20a)

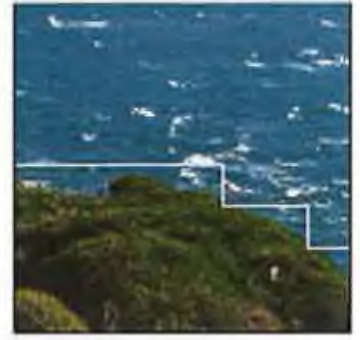

(MIII-DCT-N5a)

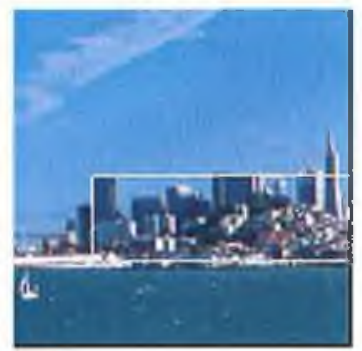

(MIII-DCT-N7a)

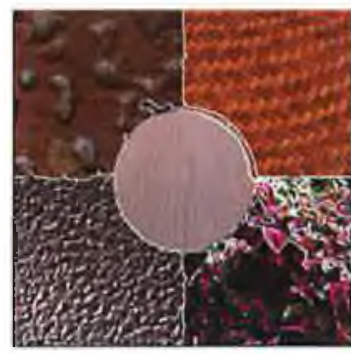

(MIII-DCT-18b)

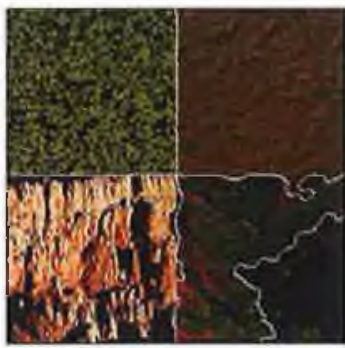

(MIII-DCT-20b)

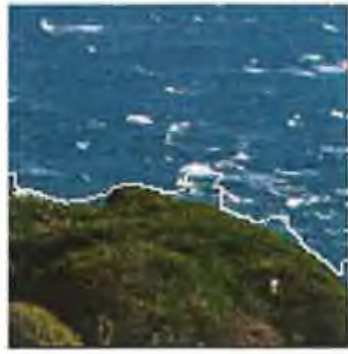

(MIII-DCT-N5b)

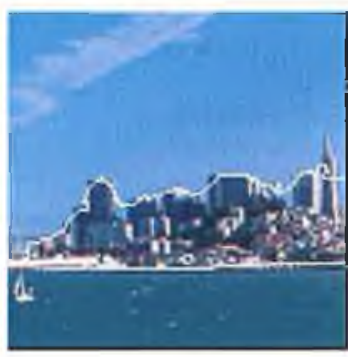

(MIII-DCT-N7b)

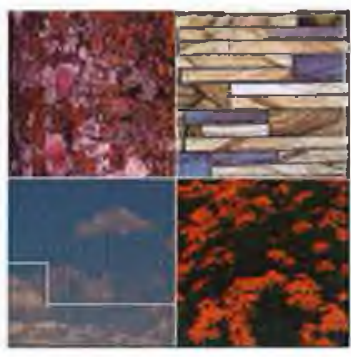

(MIII-DCT-19a)

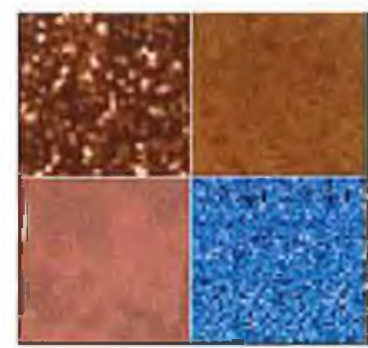

(MIII-DCT-21a)

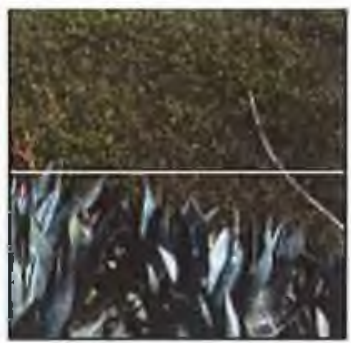

(MIII-DCT-N6a)

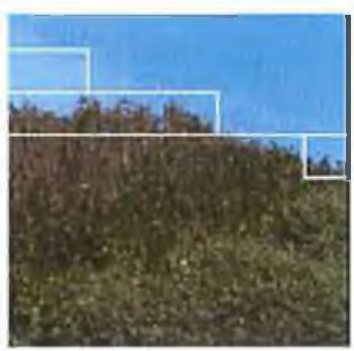

(MIII-DCT-N8a)

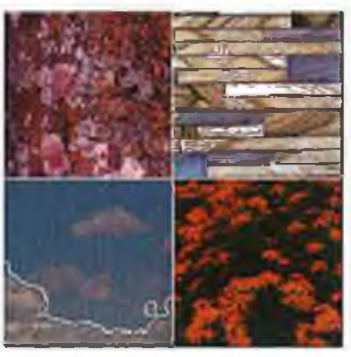

(MIII-DCT-19b)

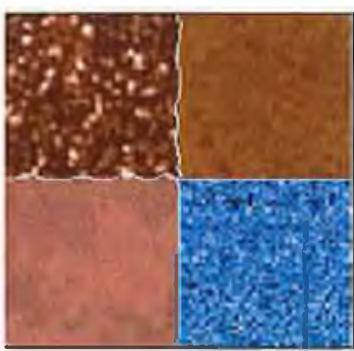

(MIII-DCT-21b)

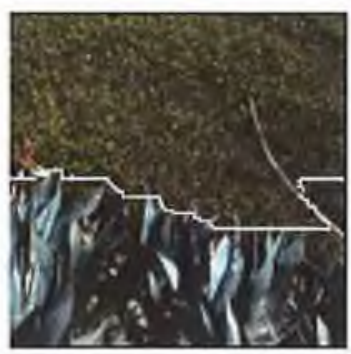

(MIII-DCT-N6b)

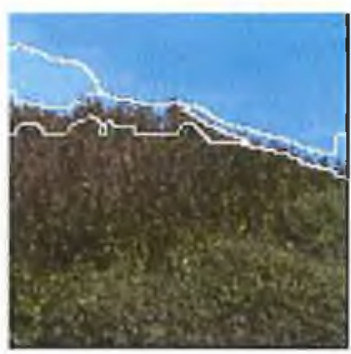

(MIII-DCT-N8b)

Figure G.6: Sample segmented results of mosaics and natural images from Method-III using DCT with colour clustering 